\title{
Pd(II)-Catalyzed Enantioselective Desymmetrization of Polycyclic Cyclohexenediones: Conjugate Addition vs. Oxidative Heck
}

\author{
Claire J. C. Lamb, Filipe Vilela and Ai-Lan Lee*
}

Institute of Chemical Sciences, Heriot-Watt University, Edinburgh EH14 4AS, Scotland, U. K.

Supporting Information 


\section{Contents}

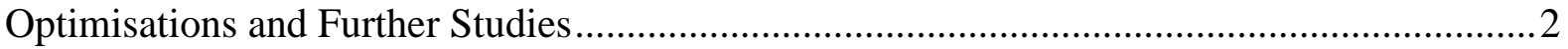

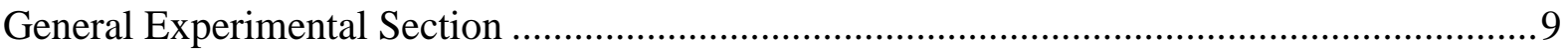

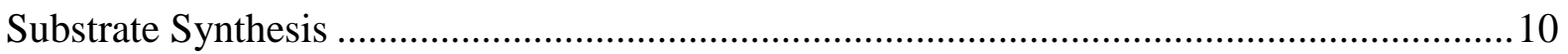

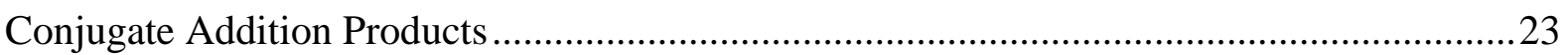

General Racemic Reaction Procedure …................................................................. 23

General Enantioselective Reaction Procedure A …........................................................2 23

General Enantioselective Reaction Procedure B ….....................................................2

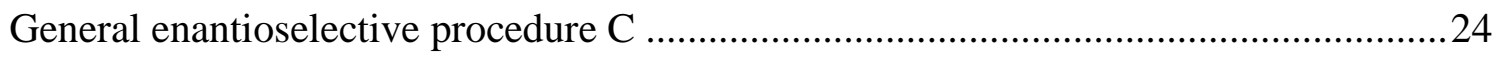

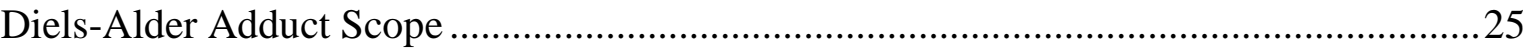

Boronic Acid Screen Conjugate Addition products........................................................... 44

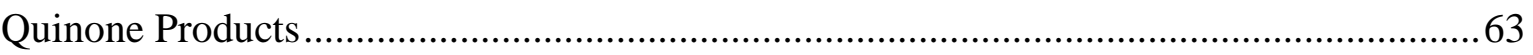

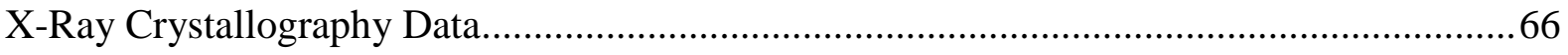

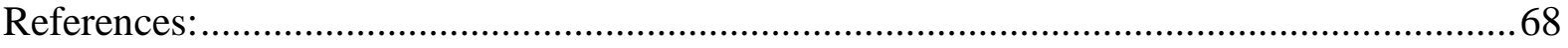

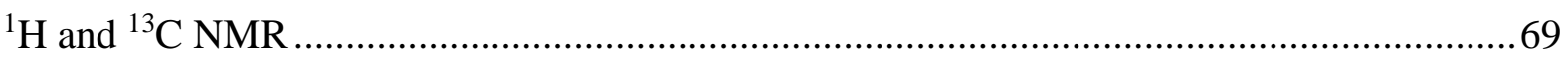




\section{Optimisations and Further Studies}

\section{Optimisation Studies of Tetramethyl Diels-Alder Adduct $4 \mathrm{f}$}

\begin{tabular}{|c|c|c|c|c|c|c|}
\hline \multicolumn{7}{|c|}{ Table S-1: Optimisation of Diels-Alder adduct $\mathbf{4 f}$} \\
\hline & & $\mathrm{Me}{ }_{\mathbf{M f}} \mathrm{OMe}$ & \multicolumn{2}{|c|}{$\begin{array}{l}\text { Conditions A: } \\
\mathrm{Pd}(\mathrm{OAc})_{2}(5 \mathrm{~mol} \%) \\
{ }^{\mathrm{B}} \mathrm{BuPyOx} \times(6 \mathrm{~mol} \%) \\
\text { DMF, } \mathrm{O}_{2}, 40{ }^{\circ} \mathrm{C}, 72 \mathrm{~h} \\
\text { Conditions } \mathrm{B}: \\
\mathrm{Pd}(\mathrm{TFA})_{2}(5 \mathrm{~mol} \%) \\
{ }^{t} \mathrm{BuPyOx} \text { ligand }(6 \mathrm{~mol} \%) \\
\text { DCE, } 40^{\circ} \mathrm{C}, 72 \mathrm{~h}\end{array}$} & \multicolumn{2}{|r|}{$\mathrm{Me}$} \\
\hline Entry & $\begin{array}{l}\text { Condit } \\
\text { ions }\end{array}$ & Ligand & $\begin{array}{l}\text { Yield }(\%)^{\mathrm{a}} \\
\mathbf{4 f : 5 f}\end{array}$ & $\begin{array}{l}\text { Isolated } \\
\text { Yield } \\
(\%)\end{array}$ & e.r. ${ }^{b}$ & Comments \\
\hline 1 & $\mathrm{~A}$ & ${ }^{t} \mathrm{BuPyOx} \quad \mathbf{L}-\mathbf{A}$ & $20: 30$ & - & - & \\
\hline $2^{\mathrm{c}}$ & A & ${ }^{t} \mathrm{BuPyOx} \quad \mathbf{L}-\mathbf{A}$ & $-: 43$ & - & $97: 3$ & $\sim 90 \%$ clean \\
\hline 3 & $\mathrm{~B}$ & 4- $\mathrm{CF}_{3}{ }^{t} \mathrm{BuPyOx} \quad \mathbf{L}-\mathbf{C}$ & $20: 67$ & 67 & $92: 8$ & \\
\hline 4 & $\mathrm{~B}$ & 4- $\mathrm{CF}_{3}{ }^{t} \mathrm{BuPyOx} \quad \mathbf{L}-\mathbf{C}$ & $18: 64$ & - & - & Recrystalised 2a \\
\hline 5 & $\mathrm{~B}$ & 4- $\mathrm{CF}_{3}{ }^{t} \mathrm{BuPyOx} \quad \mathbf{L}-\mathbf{C}$ & $30: 42$ & - & - & 2a straight from bottle \\
\hline 6 & $\mathrm{~B}$ & 4- $\mathrm{CF}_{3}{ }^{t} \mathrm{BuPyOx} \quad \mathbf{L}-\mathbf{C}$ & $34: 43$ & - & - & Arylboroxine used \\
\hline 7 & B & ${ }^{t} \mathrm{BuPyOx} \quad$ L-A & $24: 61$ & 61 & $90: 10$ & \\
\hline 8 & B & 5-CF ${ }_{3}{ }^{t}$ BuPyOx L-B & $13: 69$ & 68 & $95: 5$ & \\
\hline
\end{tabular}

${ }^{\text {a }}$ Yield determined by ${ }^{1} \mathrm{H}$ NMR analysis using 1,3,5-trimethoxybenzene as internal standard, ${ }^{\text {b}}$ E.r. determined by chiral stationary phase HPLC (CSP-HPLC), ${ }^{\circ}$ Reaction carried out with $\mathrm{Pd}(\mathrm{OAc})_{2}(10$ mol\%) and ${ }^{t} \mathrm{BuPyOx}$ L-A (11 mol\%),

Tetramethyl Diels-Alder adduct $\mathbf{5 f}$ behaved unexpectedly when optimised enantioselective conditions were applied (Table S-1, entries 1-2). Despite the racemic reaction (using 1,10phenanthroline as ligand) furnishing the product in a 77\% yield, applying the optimised enantioselective conditions A only yielded $30 \%$ yield of desired product (entry 1 ) by ${ }^{1} \mathrm{H} \mathrm{NMR}$ analysis and increasing catalyst and ligand loading to 10/11 mol\% only slightly improved the yield to $43 \%$ (entry 2). Furthermore, the product could not be isolated pure as it co-eluted with unidentified side products. Despite this, an excellent e.r. of 97:3 was recorded.

To address this, more typical conjugate addition coupling conditions were investigated: $\mathrm{Pd}(\mathrm{TFA})_{2}(5 \mathrm{~mol} \%),{ }^{t} \mathrm{BuPyOx}$ ligands $(6 \mathrm{~mol} \%)$, dichloroethane (DCE) as solvent at $40{ }^{\circ} \mathrm{C}$ for 72 h. $^{1}$ Pleasingly, this improved the yield significantly (Table S-1, entries 3, $7-8$ ) with 5$\mathrm{CF}_{3}{ }^{t} \mathrm{BuPyOx} \mathbf{L}-\mathbf{B}$ proving to be the optimal ligand furnishing the conjugate addition product with 68\% yield and an e.r. of 95:5. Interestingly, under conditions A for the majority of arylboronic acids screened, the arylboronic acid $\mathbf{2}$ could be used straight from the bottle (which would be an equilibrium mixture of the boronic acid and boroxine) ${ }^{2}$ with no detriment to yield. However, under conditions $\mathrm{B}$, there is a strong preference for the use of the recrystallised arylboronic acid 2 (entry 4, 64\%), vs. using arylboronic acid straight from the bottle (entry 5, $42 \%$ ) or dehydrating the boronic acid to the corresponding arylboroxine (entry $6,43 \%$ ). 


\section{Studies with anthracene Diels-Alder Adduct 4h}

The Diels-Alder adduct of benzoquinone and anthracene (4h) behaved quite differently compared to all other substrate during initial catalytic studies. Application of optimised racemic coupling conditions at $40{ }^{\circ} \mathrm{C}$ resulted in a mixture of both the conjugate addition and the oxidative Heck product in a 10:1 ratio. Increasing the reaction temperature to $100{ }^{\circ} \mathrm{C}$ gave the benzoquinone product 7 at $43 \%$ yield, whilst $70{ }^{\circ} \mathrm{C}$ gave a mixture of the benzoquinone coupled product 7 and the oxidative Heck product 6h' (Scheme S-1).

Of all the substrates $\mathbf{4}$ tested, $\mathbf{4 h}$ was the only one that showed any oxidative Heck product under these conditions. For example, substrate $4 \mathbf{a}$ was also subjected to the same conditions at $70{ }^{\circ} \mathrm{C}$ and $100{ }^{\circ} \mathrm{C}$. At $70{ }^{\circ} \mathrm{C}, 40 \%$ conjugate addition product was observed, along with $20 \%$ retro-Diels-Alder, and other unidentified products, while at $100{ }^{\circ} \mathrm{C}, 100 \%$ retro-Diels-Alder of 4a was observed.<smiles>O=C1C=CC(=O)C2C3CC(C4C=CC(C5CCCC5)C43)C12</smiles>

4h<smiles>COc1ccc(B(O)O)cc1</smiles>

2a

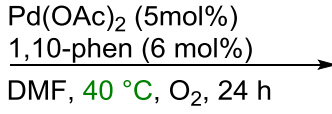

DMF, $40{ }^{\circ} \mathrm{C}, \mathrm{O}_{2}, 24 \mathrm{~h}$

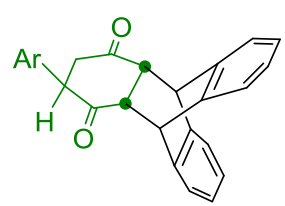

$5 h^{\prime}$

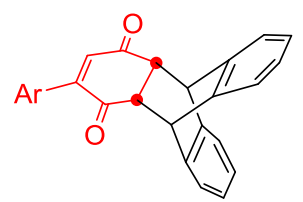

$6 h^{\prime}$

$$
\begin{gathered}
\text { Conjugate } \\
\text { addition }
\end{gathered} \quad 10: 1 \text { "Heck-type" }
$$<smiles>O=C1C=CC(=O)C2C3CC(C4C=CC3C3CCCC43)C12</smiles>

$4 h$<smiles>OBO</smiles><smiles>COc1ccc(C)cc1</smiles>

2a
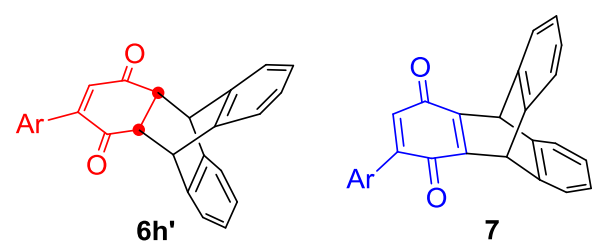

"Heck-type" 1 : 2 Fully oxidised

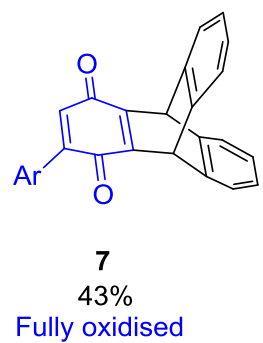

Scheme S-1: Investigations with anthracene Diels-Alder adduct $4 \mathrm{~h}$ (racemic conditions). 


Table S-2: Enantioselective optimisation of Diels-Alder adduct 4h
Conditions $\mathrm{A}$ :
Entry

${ }^{\text {a }}$ Yield determined by ${ }^{1} \mathrm{H}$ NMR analysis using 1,3,5-trimethoxybenzene as internal standard, ${ }^{\text {b}}$ E.r. determined by chiral stationary phase HPLC, 'Not determined; $46 \%$ combined yield of $\mathbf{5 h}$ ' and $\mathbf{6 h}$ ', ${ }^{\mathrm{d}}$ Isolated yield

Unexpectedly, when a chiral ${ }^{t} \mathrm{BuPyOx}$ L-A was employed during enantioselective coupling, the oxidative Heck product $\mathbf{6}$ ' ' was observed as the main product. CSP-HPLC separating conditions could not be achieved for $p$-methoxy substitution of the aryl boronic acid $\mathbf{2 a}$ (Table S-2, entry 1), so $p$-hydroxy aryl boronic acid $\mathbf{2 m}$ was used instead as the racemic oxidative Heck/conjugate addition mixture could be separated by CSP-HPLC. Using our previously optimised conditions A (Table S-2, entries 2 - 5), we obtained good yields for oxidative Heck but very poor yields for conjugate addition. However, the mixtures of conjugate addition $\mathbf{5 h}$ and oxidative Heck $\mathbf{6}$ h products could not be separated by silica gel column chromatography and therefore, the previously optimised conditions A were not useful for this substrate. Furthermore, carrying the reaction out in air instead of oxygen does not promote conjugate addition over oxidative Heck, instead the yield dramatically drops for both reactions (Table S2 , entries $2-3)$.

We therefore decided to investigate conditions more typically utilised for $\mathrm{Pd}(\mathrm{II})$-catalysed conjugate addition reactions, ${ }^{1}$ employing $4-\mathrm{CF}_{3}{ }^{t} \mathrm{BuPyOx} \mathbf{L}-\mathbf{C}$ ligand $(6 \mathrm{~mol} \%)$ in the presence 
of $\mathrm{Pd}(\mathrm{TFA})_{2}(5 \mathrm{~mol} \%)$ in DCE at $40{ }^{\circ} \mathrm{C}$ furnished exclusively the conjugate addition product in 65\% isolated yield and good e.r. of 85:15 (Table S-2, Conditions B, entry 6).

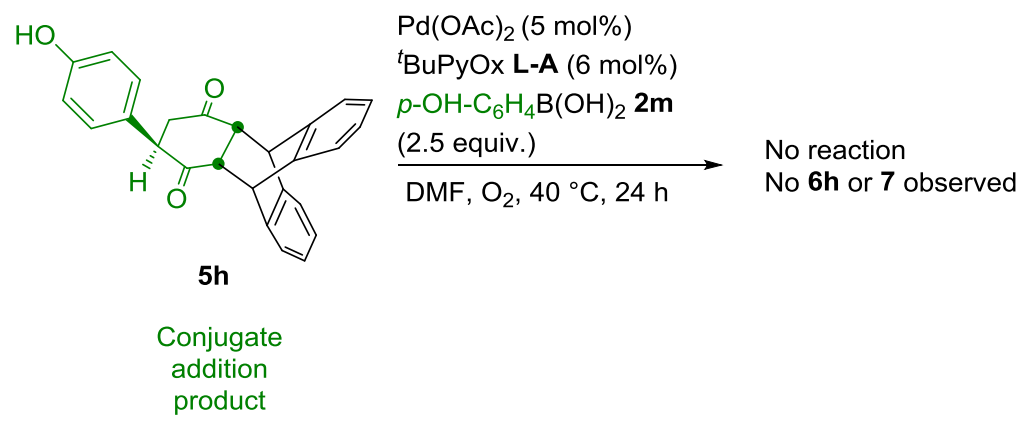

Scheme S2: Control reaction

As a control, conjugate addition product $\mathbf{5 h}$ was re-subjected to conditions A (Scheme S2), to establish if $\mathbf{6 h}$ was the product of an oxidative Heck reaction or conjugate addition followed by dehydrogenation. After $24 \mathrm{~h}$, no trace of the formal oxidative Heck product was observed, implying that $\mathbf{6 h}$ is the result of a true oxidative Heck reaction. Furthermore, no double oxidation product benzoquinone 7 was observed in the control reaction.

\section{Diels-Alder Adduct 4i Studies}

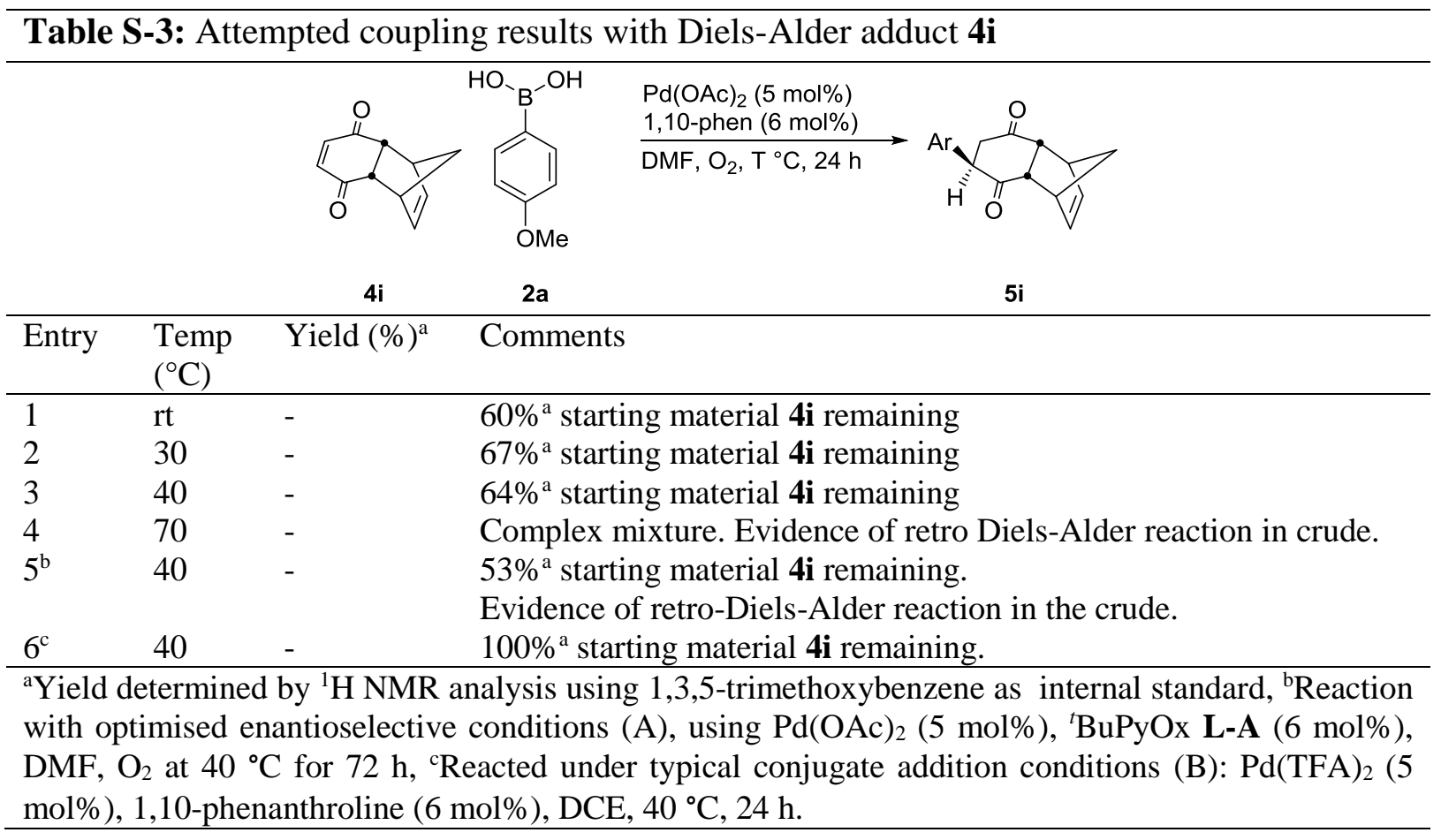

Several attempts were made to try desymmetrise Diels-Alder adduct $4 \mathbf{i}$ with $p$-methoxyphenyl boronic acid 2a. Unfortunately, starting material was the only identifiable component of the reaction in each reaction's crude mixture (determined by ${ }^{1} \mathrm{H}$ NMR) when using optimised racemic conditions (Table S-3, entries 1-3). Increasing the reaction temperature failed to yield desired product and instead only produced a complex mixture both by TLC and NMR analysis of the crude mixture, with retro Diels-Alder adduct product $p$-benzoquinone being observed in the crude NMR (Table S-3, entry 4). For completeness, optimised enantioselective conditions 
were also applied, but again failed to furnish desired product (Table S-3, entry 5). Finally, more typical racemic conjugate addition reactions were also applied to no effect (Table S-3, entry $6)$.

We hypothesised that this unexpected lack of reactivity with substrate $4 \mathbf{i}$ may be due to the fact that it may act as a bidentate diene ligand for Pd(II), via the unsubstituted alkene and the enedione, thereby deactivating the catalyst. In order to probe this, we performed a ${ }^{1} \mathrm{H} N M R$ study: $\mathrm{Pd}(\mathrm{OAc})_{2}{ }^{t}{ }^{\mathrm{BuPyO}} \mathbf{\mathbf { L }}-\mathbf{A}$ catalyst was formed in solution, and Diels-Alder adduct $\mathbf{4 i}$ was subsequently added (Figure S-1, red triangle). This NMR spectra was overlaid with the free Diels-Alder adduct $4 \mathbf{i}$ (Figure S-1, blue circle), normalising to the $\mathrm{CHCl}_{3}$ signal at $7.26 \mathrm{ppm}$. A slight shift in the alkene $\mathrm{H}_{\mathrm{A}}$ and $\mathrm{H}_{\mathrm{B}}$ signals is observed, providing some evidence of chelation of $4 \mathbf{i}$ to $\mathrm{Pd}(\mathrm{II})$.

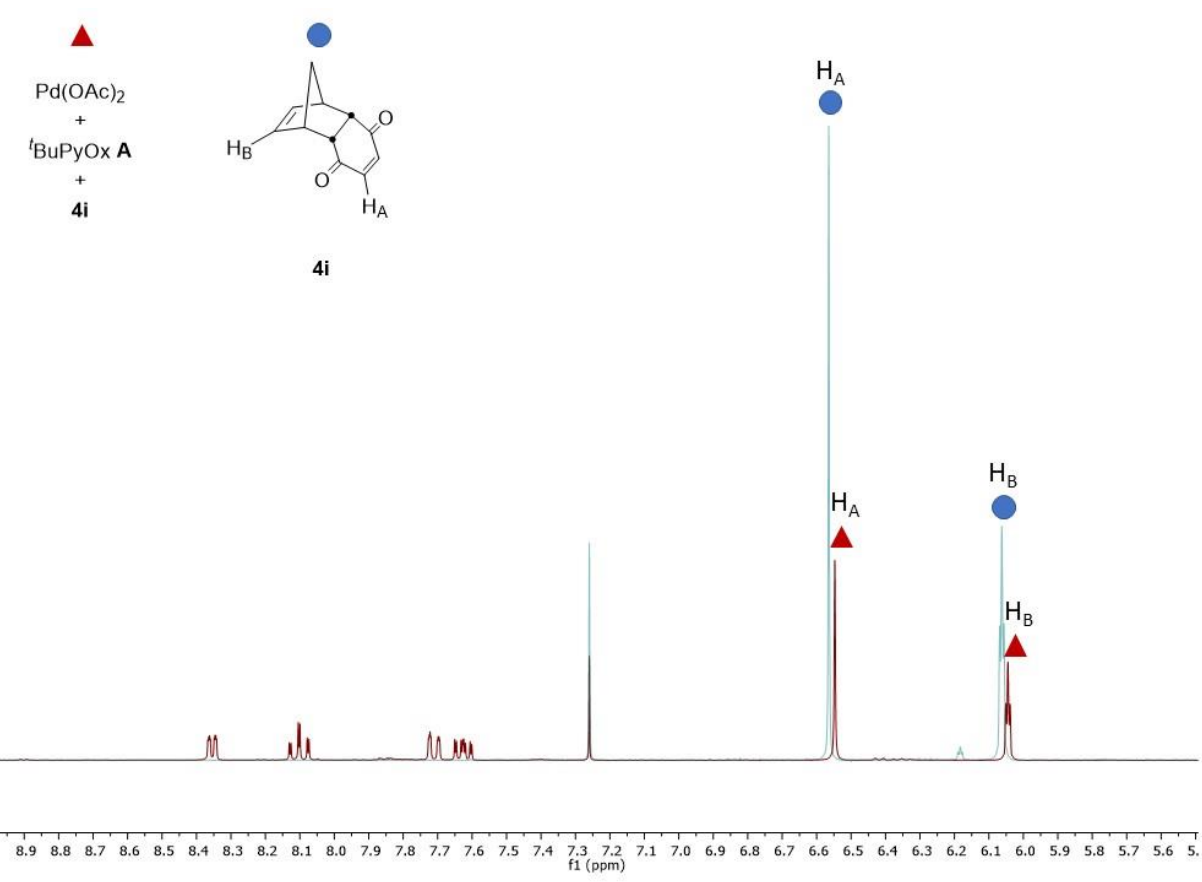

Figure S-1: ${ }^{1} \mathrm{H}$ NMR investigations of Diels-Alder adduct $4 \mathbf{i}\left(300 \mathrm{MHz}\right.$ in $\left.\mathrm{CDCl}_{3}\right)$ 


\section{Investigations with Quinone Substrate 8}

Several unsuccessful attempts were made to selectively reduce the more electron-rich alkene in the Diels-Alder adduct $4 \mathbf{i}$ to give the corresponding saturated enedione, in order to test whether removing the more electron-rich alkene in $\mathbf{4} \mathbf{i}$ would restore reactivity. Nevertheless, we were able to synthesise quinone substrate $\mathbf{8}$ (via full reduction of both alkenes in $\mathbf{4 i}$ and subsequent dehydrogenation to $\mathbf{8}$ ) for investigations.

\begin{tabular}{llllll}
\hline \multicolumn{5}{c}{ Table S-4: Investigations with quinone substrate 8} \\
\hline
\end{tabular}

${ }^{\mathrm{a}}$ Isolated yield. ${ }^{\mathrm{b}}$ E.r. determined by chiral stationary phase HPLC. ${ }^{\mathrm{c}} \mathrm{Pd}(\mathrm{OAc})_{2}(5 \mathrm{~mol} \%)$ and 1,10-phenanthroline $(5 \mathrm{~mol} \%)$. ${ }^{\mathrm{d}}$ Yield determined by ${ }^{1} \mathrm{H}$ NMR analysis using 1,3,5trimethoxybenzene as internal standard. ${ }^{\mathrm{e}} 72 \mathrm{~h}$.

Enantioselective oxidative Heck coupling conditions were developed for the desymmetrisation of 8 to 9. DCBQ was required to obtain good conversion to the oxidative Heck coupled product 9 (Table S-4, entry 1 v. 2). Despite oxygen being present in the system, the quinone starting material 8 and the quinone product 9 could also participate in the oxidation of $\mathrm{Pd}(0) \rightarrow \operatorname{Pd}(\mathrm{II})$ catalyst and thus be reduced to the corresponding hydroquinones, so DCBQ was added as oxidant.

A mini-optimisation was carried out with ${ }^{t} \mathrm{BuPyOx} \mathbf{L}-\mathbf{A}$ and $\mathbf{L}-\mathbf{B}$, along with varying temperature, however an e.r. of 65:35 (Table $S-4$, entry 6) was the best that could be achieved, albeit with an excellent yield of $96 \%$ (entry 5). 


\section{Proposed Rationalisation of Observed Stereochemistry}

Based on computational modelling and mechanistic investigations by Stoltz et al. ${ }^{3}$ into enantioselective conjugate addition reactions with related cyclohexanone substrates and $\mathrm{Pd}(\mathrm{II})^{t} \mathrm{BuPyOx}$ catalyst, we postulate that TS-A during the migratory insertion step, leads to the observed stereochemistry of coupled product 5 (Figure S-2). The computational modelling within Stoltz's investigations suggests it is energetically favourable for the aryl group to transmetallate trans to the tert-butyl group of the chiral oxazoline ligand (to avoid steric clash of the aryl and ${ }^{t} \mathrm{Bu}$ groups), ${ }^{3}$ thereby precluding TS-C and TS-D as options. In TS-B, the wrong enantiomer would be formed, and this TS is unfavourable because of steric clashes with the top of the bicyclic bridge and the tert-butyl group of the chiral ligand. TS-A avoids this steric clash by having the steric bulk removed from the tert-butyl group and results in the observed $(R)$ geometry with respect to the stereocentre formed during the conjugate addition reaction.

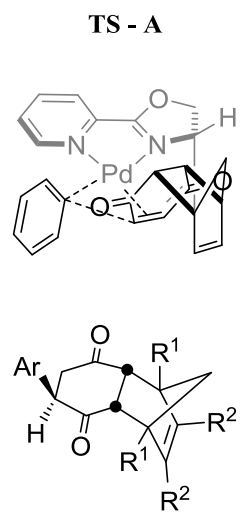

(R) enantiomer at reaction site

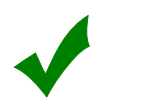

TS - B
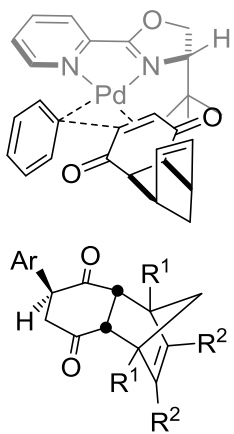

(S) enantiomer at reaction site
TS - C
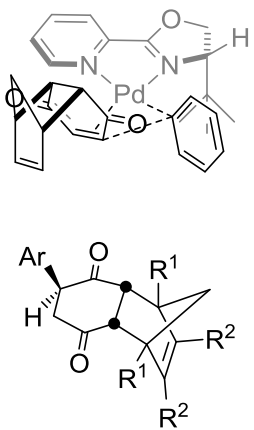

(S) enantiomer at reaction site

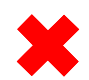

TS - D

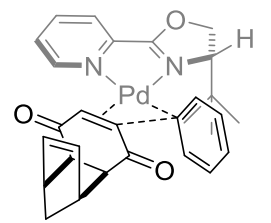

(R) enantiomer at reaction site

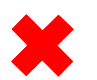

Figure S-2: Proposed transition states (substitution removed for clarity) for the migratory insertion step 


\section{General Experimental Section}

${ }^{1} \mathrm{H}$ NMR spectra were recorded on Bruker AV 300 and AV 400 spectrometers at 300 and 400 $\mathrm{MHz}$ respectively and referenced to residual solvent. ${ }^{13} \mathrm{C}$ NMR spectra were recorded using the same spectrometers at 75 and $100 \mathrm{MHz}$ respectively. Chemical shifts ( $\delta$ in ppm) were referenced to residual solvent peaks $\left(\mathrm{CDCl}_{3}\right.$ at $\delta_{\mathrm{H}} 7.26, \delta_{\mathrm{C}}$ at $77.16 \mathrm{ppm}$.)

$J$ values are given in $\mathrm{Hz}$ and s, d, dd, t, q, qn and $\mathrm{m}$ abbreviations correspond to singlet, doublet, doublet of doublet, triplet, quartet, quintet and multiplet. Mass spectra were obtained at the EPSRC UK National Mass Spectrometry Facility at Swansea University. Infrared spectra were obtained on Perkin-Elmer Spectrum 100 FT-IR Universal ATR Sampling Accessory, deposited neat or as a chloroform solution to a diamond/ZnSe plate. Flash column chromatography was carried out using Matrix silica gel 60 from Fluorochem and TLC was performed using Merck silica gel 60 F254 pre-coated sheets and visualised by UV (254 $\mathrm{nm})$ or stained by the use of aqueous acidic $\mathrm{KMnO} 4$ or aqueous acidic ceric ammonium molybdate as appropriate. Enantiomer separation was achieved by chiral stationary phase HPLC (CSP-HPLC) with an Agilent Technologies 1120 Compact LC with either CHIRALPAK IA, IB or IC column as appropriate. Optical rotation was measured on a Bellingham and Stanley ADP410 polarimeter. Pet. ether refers to petroleum ether $\left(40-60{ }^{\circ} \mathrm{C}\right)$. Anhydrous DMF was obtained from a solvent purification system. All arylboronic acids were purchased from Sigma-Aldrich, Fluorochem or Acros. In most circumstances, the aryl boronic acid could be used directly from the bottle. In specific cases where the corresponding boroxine or recrystallised boronic acids performed better, this is documented in the relevant reaction. The coupling reactions were carried out in dried glassware, using either anhydrous DMF from a solvent purification system and $\operatorname{Pd}(\mathrm{OAc})_{2}$ from Johnson Matthey, or using DCE from Arcos stored over $4 \AA$ MS and Pd(TFA) 2 synthesised according to literature procedure with minor modifications. ${ }^{4}$ 


\section{Substrate Synthesis}

\section{1,2,3,4-Tetrachloro-9,9-dimethoxy-1,4,4a,8a-tetrahydro-1,4-methanonaphthalene-5,8- dione $^{5} 4 a$}

Synthesises according to modified literature procedure..$^{5}$
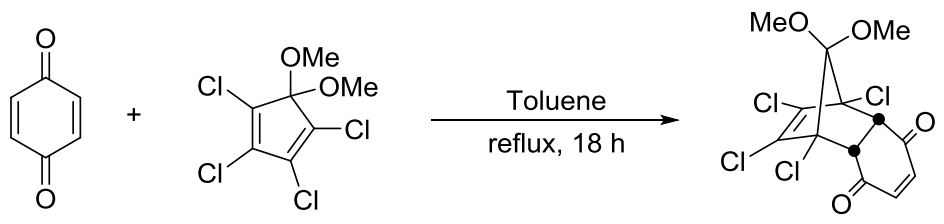

$4 a$

To oven dried reflux apparatus, back-filled with argon, 1,2,3,4-tetrachloro-5,5dimethoxycyclopentadiene (498.7 mg, $1.89 \mathrm{mmol}, 1.00$ equiv.) was stirred in toluene for 5 minutes before benzoquinone (209.1 $\mathrm{mg}, 1.93 \mathrm{mmol}, 1.02$ equiv.) was added and the reaction was stirred at reflux for $18 \mathrm{~h}$. The reaction was cooled, and solvent was removed under reduced pressure. The resulting residue was purified by silica gel column chromatography $(12: 1 \rightarrow 5: 1$ pet. ether: EtOAc) to yield $4 \mathbf{a}(610.0 \mathrm{mg}, 1.64 \mathrm{mmol}, 87 \%)$ as a crystalline yellow powder.

Mp: $160-163{ }^{\circ} \mathrm{C}$ (ethyl actetate/pet. ether) (literature mp: $156-158{ }^{\circ} \mathrm{C}$ ) ${ }^{5}$; $\mathrm{R}_{\mathrm{f}}: 0.2$ in $12: 1$ pet. ether:EtOAc; $v_{\max } / \mathrm{cm}^{-1}: 3025,2932,1674,1014,736 ;{ }^{1} \mathrm{H}$ NMR $\left(300 \mathrm{MHz}, \mathrm{CDCl}_{3}\right) \delta 6.72(\mathrm{~s}$, $2 \mathrm{H},=\mathrm{CH}), 3.66\left(\mathrm{~s}, 3 \mathrm{H}, \mathrm{OCH}_{3}\right), 3.63(\mathrm{~s}, 2 \mathrm{H}, \mathrm{CH}), 3.60\left(\mathrm{~s}, 3 \mathrm{H}, \mathrm{OCH}_{3}\right) ;{ }^{13} \mathrm{C} \mathrm{NMR}(75 \mathrm{MHz}$, $\left.\mathrm{CDCl}_{3}\right) \delta 191.8(\mathrm{C}), 142.2(\mathrm{CH}), 129.5(\mathrm{C}), 111.2(\mathrm{C}), 77.9(\mathrm{C}), 54.9(\mathrm{CH}), 53.4\left(\mathrm{CH}_{3}\right), 52.4$ $\left(\mathrm{CH}_{3}\right)$. 
(3,5-Dimethylcyclopenta-2,5-diene-1,2-diyl)dibenzene ${ }^{6}$ S5

Synthesised according to a modified literature procedure. ${ }^{6}$
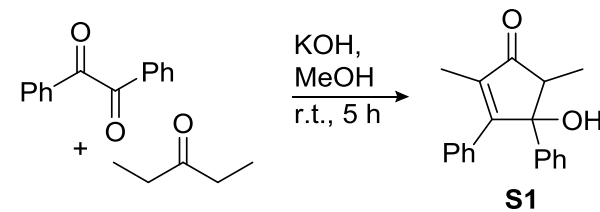

S1

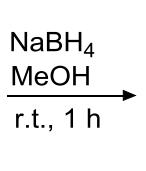$$
\mathrm{Ph}
$$

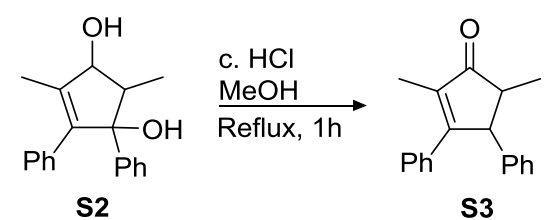

S3

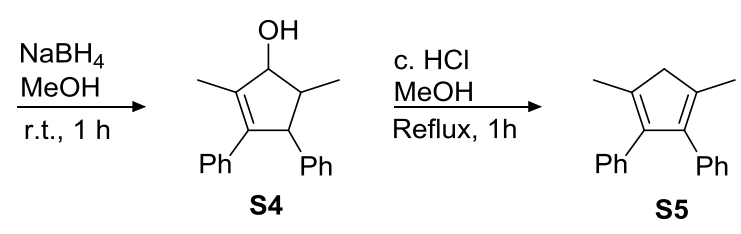

Benzil (4.21 g, 20.0 mmol, 1.0 equiv.) and pentan-3-one (1.73 g, $20.0 \mathrm{mmol}, 1.0$ equiv.) were dissolved in ethanol absolute $(10 \mathrm{~mL})$, potassium hydroxide pellets $(218.6 \mathrm{mg}, 3.0 \mathrm{mmol}, 0.15$ equiv.) were added and the reaction was stirred at room temperature for $16 \mathrm{~h}$. The reaction was then acidified with $\mathrm{HCl}(1 \mathrm{M})$ and extracted with EtOAc $(3 \times 20 \mathrm{~mL})$. The combined organic layers were washed with brine, dried over $\mathrm{MgSO}_{4}$ and solvent was removed under reduced pressure to yield S1 (5.15 g, $18.5 \mathrm{mmol}, 92 \%)$ as a pale-yellow powder and as mixture of diastereomers. The crude product was taken forward without further purification.

S1 (3.80 g, 13.7 mmol, 1.0 equiv.) was dissolved in methanol (40 mL), $\mathrm{NaBH}_{4}(574.3 \mathrm{mg}, 15.0$ mmol, 1.1 equiv.) was added in portions and the reaction was stirred at room temperature for $1 \mathrm{~h}$. The reaction was quenched with water $(2.5 \mathrm{~mL})$ and extracted with DCM $(3 \times 20 \mathrm{~mL})$, the combined organic layers were washed with brine and dried over $\mathrm{MgSO}_{4}$. Solvent was removed under reduced pressure to yield $\mathbf{S 2}(3.50 \mathrm{~g}, 12.5 \mathrm{mmol}, 88 \%)$ as an off-white powder and as a mixture of diastereomers. The crude product was taken forward without further purification.

S2 (5.40 g, $19.2 \mathrm{mmol}, 1.0$ equiv.) was dissolved in methanol (40 mL) and conc. $\mathrm{HCl}(4 \mathrm{~mL})$ was added. The mixture was stirred at reflux for $1.5 \mathrm{~h}$. Upon completion, the reaction mixture was neutralised with $\mathrm{NaOH}(1 \mathrm{M})$ and extracted with $\mathrm{DCM}(3 \times 20 \mathrm{~mL})$ and the combined organic layers were washed with brine and dried over $\mathrm{MgSO}_{4}$. Solvent was removed under reduced pressure. The resulting crude product was purified by recrystallisation with DCM/Petrol to yield S3 (5.02 g, $19.1 \mathrm{mmol}, 99 \%)$ as white crystalline powder and as a mixture of diastereomers.

S3 (4.00 g, 15.2 mmol, 1.0 equiv.) was dissolved in methanol (40 mL), $\mathrm{NaBH}_{4}(638.0 \mathrm{mg}, 16.9$ mmol, 1.1 equiv.) was added in portions and the reaction was stirred at room temperature for $1 \mathrm{~h}$. The reaction was quenched with water $(2.5 \mathrm{~mL})$ and extracted with DCM $(3 \times 20 \mathrm{~mL})$, the 
combined organic layers were washed with brine and dried over $\mathrm{MgSO}_{4}$ and solvent was removed under reduced pressure. The resulting crude was recrystallised with hot hexanes to yield S4 (3.90 g, $14.7 \mathrm{mmol}, 97 \%)$ as white crystalline prisms and as a mixture of diastereomers.

S4 (4.00 g, $15.1 \mathrm{mmol}, 1.0$ equiv.) was dissolved in methanol $(50 \mathrm{~mL})$ and conc. $\mathrm{HCl}(5 \mathrm{~mL})$ was added. The mixture was stirred at reflux for $1.5 \mathrm{~h}$. The reaction mixture was then neutralised with $\mathrm{NaOH}(1 \mathrm{M})$ and transferred to a separating funnel. The organic layer was extracted with DCM $(3 \times 20 \mathrm{~mL})$ and the combined organic layers were washed with brine and dried over $\mathrm{MgSO}_{4}$. Solvent was removed under reduced pressure. The resulting crude product was purified by recrystallisation with hot methanol to yield S5 (3.50 g, $14.2 \mathrm{mmol}, 94 \%)$ as yellow crystalline needles.

Mp: $87{ }^{\circ} \mathrm{C}$ (methanol) (literature mp: $\left.89-90{ }^{\circ} \mathrm{C}\right){ }^{6} \mathrm{R}_{\mathrm{f}}: 0.9$ in pet. ether; $v_{\max } / \mathrm{cm}^{-1}: 2905,2850$, 1603, 1494, 755, 703; ${ }^{1} \mathrm{H}$ NMR (300 MHz, $\left.\mathrm{CDCl}_{3}\right) \delta 7.25-7.13$ (m, 6H, Ar-H), 7.00 - 6.92 $(\mathrm{m}, 4 \mathrm{H}, \mathrm{Ar}-\mathrm{H}), 3.14\left(\mathrm{~s}, 2 \mathrm{H}, \mathrm{CH}_{2}\right), 2.06\left(\mathrm{~s}, 6 \mathrm{H}, \mathrm{CH}_{3}\right) ;{ }^{13} \mathrm{C} \mathrm{NMR}\left(75 \mathrm{MHz}, \mathrm{CDCl}_{3}\right) \delta 141.6(\mathrm{C})$, $136.8(\mathrm{C}), 136.5(\mathrm{C}), 129.7(\mathrm{CH}), 127.7(\mathrm{CH}), 126.1(\mathrm{CH}), 50.1\left(\mathrm{CH}_{2}\right), 14.6\left(\mathrm{CH}_{3}\right)$. 


\section{1,4-Dimethyl-2,3-diphenyl-1,4,4a,8a-tetrahydro-1,4-methanonaphthalene-5,8-dione $\mathrm{5}^{\mathbf{4}} \mathbf{b}$}

Synthesised according to a modified literature procedure. ${ }^{5}$

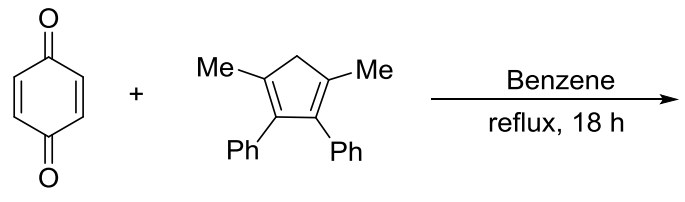

S5

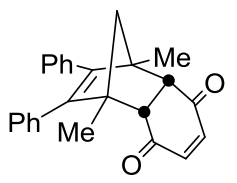

$4 b$

Benzoquinone (200 mg, $1.85 \mathrm{mmol}, 1$ equiv.) was dissolved in benzene (5 mL), S5 was then added (547 mg, $2.22 \mathrm{mmol}, 1.2$ equiv.) and the reaction was stirred at reflux for $16 \mathrm{~h}$. The reaction was then cooled, and solvent was removed under reduced pressure. The resulting crude was purified by silica gel column chromatography $(10: 1 \rightarrow 5: 1$ pet. ether/EtOAc) to yield $\mathbf{4 b}$ $(620.0 \mathrm{~g}, 1.75 \mathrm{mmol}, 95 \%)$ as a yellow powder.

Mp: $158{ }^{\circ} \mathrm{C}$ (methanol) (literature mp: $147-149{ }^{\circ} \mathrm{C}$ ) ${ }^{5}$; $\mathrm{R}_{\mathrm{f}}: 0.2$ in 10:1 pet. ether:EtOAc; $v_{\max } /$ $\mathrm{cm}^{-1}: 2930,2858,1757,1659,1038,752,700 ;{ }^{1} \mathrm{H} \mathrm{NMR}\left(300 \mathrm{MHz}, \mathrm{CDCl}_{3}\right) \delta 7.08-7.01(\mathrm{~m}$, 6H, Ar-H), $6.78-6.70$ (m, 4H, Ar-H), 6.55 (s, 2H, HC=CH), 3.16 (s, 2H, HC-CH), 1.80 (d, J $=8.6 \mathrm{~Hz}, 1 \mathrm{H}, \mathrm{C} \underline{\mathrm{HH}}), 1.59(\mathrm{~d}, J=8.6 \mathrm{~Hz}, 1 \mathrm{H}, \mathrm{CH} \underline{\mathrm{H}}), 1.49\left(\mathrm{~s}, 6 \mathrm{H}, 2 \times \mathrm{CH}_{3}\right) ;{ }^{13} \mathrm{C} \mathrm{NMR}(75 \mathrm{MHz}$, $\left.\mathrm{CDCl}_{3}\right) \delta 199.2(\mathrm{C}), 145.9(\mathrm{C}), 142.4(\mathrm{CH}), 134.9(\mathrm{C}), 129.6(\mathrm{CH}), 128.1(\mathrm{CH}), 127.0(\mathrm{CH})$, $65.1\left(\mathrm{CH}_{2}\right), 59.5(\mathrm{C}), 57.3(\mathrm{CH}), 18.4\left(\mathrm{CH}_{3}\right)$. 


\section{1,4,9-Trimethyl-2,3-diphenyl-1,4,4a,8a-tetrahydro-1,4-methanonaphthalene-5,8-dione ${ }^{5}$ $4 c$}

Synthesised according to literature procedure. ${ }^{5}$

For synthesis of $\mathbf{S 3}$, please refer to the synthesis of $\mathbf{S 5}$.

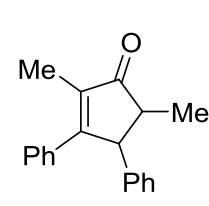

S3

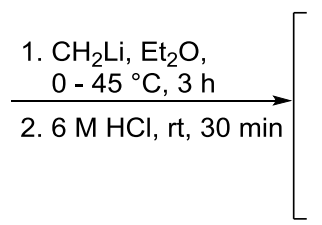

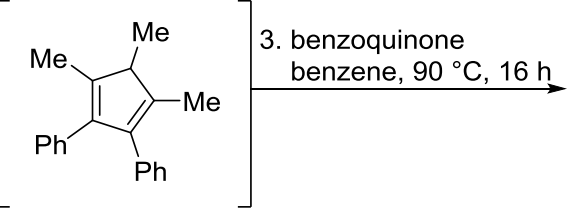

S4

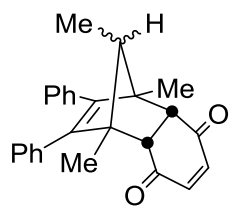

4c

To two-necked flask, oven dried and back-filled with $\mathrm{N}_{2}, \mathbf{S 3}$ (500.0 mg, $1.90 \mathrm{mmol}, 1.0$ equiv.) was dissolved in dry $\mathrm{Et}_{2} \mathrm{O}(5 \mathrm{~mL})$ and cooled to $0{ }^{\circ} \mathrm{C}$. $\mathrm{CH}_{3} \mathrm{Li}$ in $\mathrm{Et}_{2} \mathrm{O}\left(1.56 \mathrm{~mol} \mathrm{~L}^{-1}, 1.65 \mathrm{~mL}\right.$, $2.57 \mathrm{mmol}, 1.4$ equiv.) was added drop-wise before the reaction mixture was warmed to room temperature and then stirred at reflux for $3 \mathrm{~h}$. The reaction was then cooled to room temperature. $\mathrm{HCl}(6 \mathrm{M}, 3 \mathrm{~mL})$ was added dropwise and the reaction was stirred for a further 30 min. The reaction was quenched with saturated sodium bicarbonate solution $(5 \mathrm{~mL})$ and extracted with DCM $(3 \times 10 \mathrm{~mL})$, the combined organic layers were dried over $\mathrm{Na}_{2} \mathrm{SO}_{4}$ and solvent was removed under reduced pressure. The resulting crude of S4 was used in the next step without further purification due to being prone to isomerisation. ${ }^{5}$

The crude of $\mathbf{S 4}$ was transferred to a round bottom flask containing benzoquinone (173 $\mathrm{mg}$, $1.60 \mathrm{mmol}, 0.84$ equiv.) and benzene $(4 \mathrm{~mL})$ and the reaction was stirred at reflux for $16 \mathrm{~h}$. Solvent was then removed under reduced pressure. The resulting crude was purified by silica gel column chromatography $(20: 1 \rightarrow 10: 1$ pet. ether/EtOAc) to yield $4 \mathbf{c}(122.2 \mathrm{mg}, 0.33 \mathrm{mmol}$, $17 \%$ ) as a yellow powder and 7:1 mixture of diastereomers. This is consistent with the literature reported procedure and spectra. ${ }^{5}$

Mp: $129-132{ }^{\circ} \mathrm{C}$ (pet. ether:EtOAc) (literature mp: $\left.123-125{ }^{\circ} \mathrm{C}\right)^{5}$; $\mathrm{R}_{\mathrm{f}}: 0.2$ in $10: 1$ pet. ether:EtOAc; $v_{\max } / \mathrm{cm}^{-1}$ : 2970, 2927, 1659, 1602, 777, 757, 699; NMR data for the major diastereomer: ${ }^{1} \mathrm{H}$ NMR (300 MHz, $\left.\mathrm{CDCl}_{3}\right) \delta 7.15-7.06(\mathrm{~m}, 6 \mathrm{H}, \mathrm{Ar}-\mathrm{H}), 6.83-6.73(\mathrm{~m}, 4 \mathrm{H}$, Ar-H), 6.65 (s, 2H, HC=), 3.16 (s, 2H, CH), 1.81 (q, J = 6.3 Hz, 1H, CHCH$\left._{3}\right), 1.42$ (s, 6H, $\left.\mathrm{CH}_{3}\right), 0.97\left(\mathrm{~d}, J=6.3 \mathrm{~Hz}, 3 \mathrm{H}, \mathrm{CHC}_{3}\right) ;{ }^{13} \mathrm{C} \mathrm{NMR}\left(75 \mathrm{MHz}, \mathrm{CDCl}_{3}\right) \delta 199.1(\mathrm{C}), 142.6(\mathrm{C})$, $142.5(\mathrm{CH}), 135.4(\mathrm{C}), 129.3(\mathrm{CH}), 128.1(\mathrm{CH}), 126.9(\mathrm{CH}), 66.1(\mathrm{CH}), 62.4(\mathrm{C}), 57.3(\mathrm{CH})$, $16.1\left(\mathrm{CH}_{3}\right), 7.7\left(\mathrm{CH}_{3}\right)$. 


\section{1,4-Dimethyl-2,3-diphenyl-1,4,4a,8a-tetrahydro-1,4-methanonaphthalene-5,8,9-trione ${ }^{7}$ 4d}

Synthesised according to modified literature procedure. ${ }^{6,7}$

For synthesis of $\mathbf{S 1}$, please refer to the synthesis of $\mathbf{S 5}$.

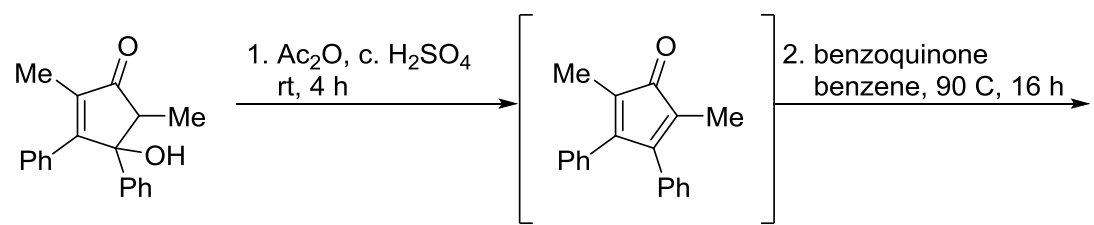

S1

S7

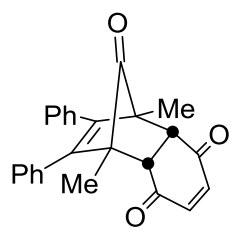

4d

A solution of $\mathbf{S 1}$ (500.0 mg, $1.80 \mathrm{mmol}, 1.0$ equiv.) in acetic anhydride (2.0 mL, $21.1 \mathrm{mmol}$, 11.7 equiv.) was stirred at room temperature for $4 \mathrm{~h}$. The bright red reaction mixture was quenched with $\mathrm{H}_{2} \mathrm{O}(1 \mathrm{~mL})$ and the precipitated product was collected by filtration and dried by reduced pressure to obtain the dimer of $\mathbf{S 7}(315.8 \mathrm{mg})$ as pale yellow-solid which was used without further purification due to being prone to dimerisation.

Crude S7 (315.8 mg, $1.2 \mathrm{mmol}, 1.0$ equiv.) was added to reflux apparatus containing benzoquinone (195.8 mg, $1.80 \mathrm{mmol}, 1.5$ equiv.) and benzene $(5 \mathrm{~mL})$ and the resulting mixture was stirred at reflux for $16 \mathrm{~h}$. Upon completion, solvent was removed under reduced pressure and the resulting crude was purified by silica gel column chromatography $(20: 1 \rightarrow 3: 1$ pet. ether/EtOAc) to yield $\mathbf{4 d}(226.5 \mathrm{mg}, 0.87 \mathrm{mmol}, 51 \%$ yield $)$ as a yellow powder.

Mp: $148-150{ }^{\circ} \mathrm{C}$ (Pet. Ether/EtOAc) (literature mp: $195-197{ }^{\circ} \mathrm{C}$ ) $;^{7}$ Rf: 0.2 in 5:1 pet. ether:EtOAc; vmax/ $\mathrm{cm}^{-1}: 3026,2928,1775,1760,1668,1611,1575,1445,807 ;{ }^{1} \mathrm{H}$ NMR (300

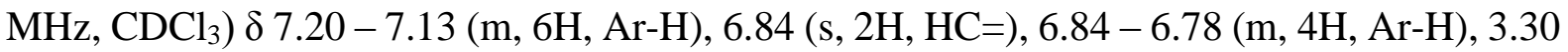
(s, 2H, CH), 1.54 (s, 6H, $\left.\mathrm{CH}_{3}\right) ;{ }^{13} \mathrm{C}$ NMR (75 MHz, CDCl3) $\delta 199.8$ (C), 195.8 (C), 143.9 $(\mathrm{CH}), 141.8(\mathrm{C}), 133.3(\mathrm{C}), 129.5(\mathrm{CH}), 128.4(\mathrm{CH}), 127.9(\mathrm{CH}), 59.0(\mathrm{C}), 52.0(\mathrm{CH}), 11.9$ $\left(\mathrm{CH}_{3}\right)$. 


\section{1,4-Diethyl-2,3-diphenyl-1,4,4a,8a-tetrahydro-1,4-methanonaphthalene-5,8,9-trione 4e}

Synthesis by modifying literature procedures. $5,6,8$

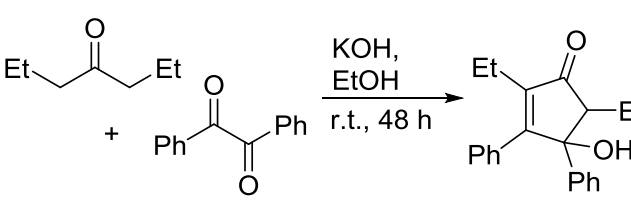

S8

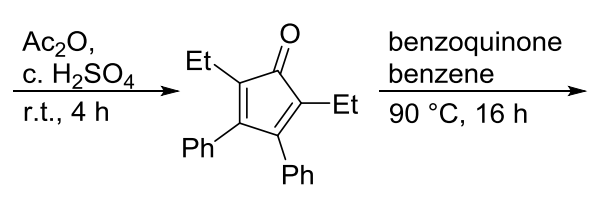

S9

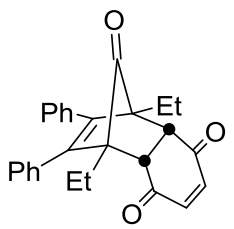

$4 e$

Benzil (2.10 g, 10.0 mmol, 1.0 equiv.) and heptantan-4-one (1.14 g, 10.0 mmol, 1.0 equiv.) were stirred in absolute ethanol $(5 \mathrm{~mL})$ at room temperature for $48 \mathrm{~h}$. Upon completion, the reaction mixture was acidified with $\mathrm{HCl}(1 \mathrm{M})$ and was extracted with EtOAc $(3 \times 15 \mathrm{~mL})$. The combined organic layers were washed with brine, dried over $\mathrm{MgSO} 4$ and solvent with removed under reduced pressure to yield $\mathbf{S 8}(2.21 \mathrm{~g}, 7.2 \mathrm{mmol}, 72 \%)$ as a pale-yellow powder and as mixture of diastereomers. The crude product was taken forward to further steps without further purification.

A solution of $\mathbf{S 8}(551.5 \mathrm{mg}, 1.8 \mathrm{mmol}, 1.0$ equiv.) in acetic anhydride (2.0 mL, $21.1 \mathrm{mmol}$, 11.7 equiv.) was stirred at room temperature for $4 \mathrm{~h}$. The bright red reaction mixture was quenched with $\mathrm{H}_{2} \mathrm{O}(1 \mathrm{~mL})$ and the precipitated product was collected by filtration and dried under reduced pressure to obtain $\mathbf{S 9}$ (392.9 mg, $1.4 \mathrm{mmol}, 76 \%)$ as bright orange solid which was used without further purification.

Crude S9 (346.1 mg, $1.2 \mathrm{mmol}, 1.0$ equiv.) was added to reflux apparatus containing benzoquinone (194.8 $\mathrm{mg}, 1.80 \mathrm{mmol}, 1.5$ equiv.) and benzene $(5 \mathrm{~mL})$ and the resulting mixture stirred at reflux for $16 \mathrm{~h}$. The solvent was then removed under reduced pressure and the resulting crude was purified by silica gel column chromatography (10:1 pet. ether/EtOAc) to yield $4 \mathbf{e}$ (226.5 mg, $0.87 \mathrm{mmol}, 51 \%$ yield) as a yellow crystalline solid.

Mp: $131-134{ }^{\circ} \mathrm{C}$ (Pet. Ether/EtOAc); Rf: 0.2 in 10:1 pet. ether:EtOAc; vmax/ $\mathrm{cm}^{-1}: 2676$, 2922, 1765, 1671, 1607, 1464, 1447, 790768,$704 ;{ }^{1} \mathrm{H}$ NMR $\left(300 \mathrm{MHz}, \mathrm{CDCl}_{3}\right) \delta=7.17-$ $7.11(\mathrm{~m}, 6 \mathrm{H}, \mathrm{Ar}-\mathrm{H}), 6.85$ (s, 2H, HC=), $6.83-6.76(\mathrm{~m}, 4 \mathrm{H}, \mathrm{Ar}-\mathrm{H}), 3.61$ (s, 2H, CH), 2.16 (dq, $J=14.6,7.4 \mathrm{~Hz}, 2 \mathrm{H}, \mathrm{C} \underline{\mathrm{HH}}), 1.93(\mathrm{dq}, J=14.6,7.4 \mathrm{~Hz}, 2 \mathrm{H}, \mathrm{CH} \underline{\mathrm{H}}), 1.02$ (t, $J=7.4 \mathrm{~Hz}, 6 \mathrm{H}$, $\left.\mathrm{CH}_{3}\right) ;{ }^{13} \mathrm{C}$ NMR (75 MHz, $\left.\mathrm{CDCl}_{3}\right) \delta=199.3(\mathrm{C}), 196.3(\mathrm{C}), 143.8(\mathrm{CH}), 142.5(\mathrm{C}), 133.7(\mathrm{C})$, 129.4 (CH), $128.3(\mathrm{CH}), 127.7(\mathrm{CH}), 62.4(\mathrm{C}), 47.2(\mathrm{CH}), 19.0\left(\mathrm{CH}_{2}\right), 9.3\left(\mathrm{CH}_{3}\right)$; HRMS (TOF MS ASAP +) $m / z$ calc. for $\mathrm{C}_{27} \mathrm{H}_{25} \mathrm{O}_{3}: 397.1804[\mathrm{M}+\mathrm{H}]^{+}$; found: 397.1799. 


\section{1,2,3,4-Tetramethyl-1,4,4a,8a-tetrahydro-1,4-methanonaphthalene-5,8-dione 4f}

Synthesised according to a modified literature procedure. ${ }^{9}$
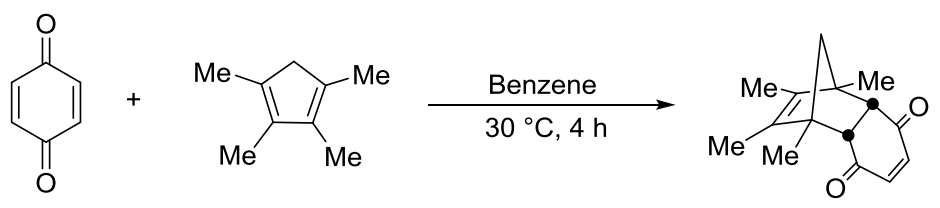

$4 \mathbf{f}$

1,2,3,4-Tetramethylcyclopentadiene ( $320 \mu \mathrm{L}, 2.30 \mathrm{mmol}, 1.0$ equiv.) was added to a flask containing benzoquinone ( $270.9 \mathrm{mg}, 2.50 \mathrm{mmol}, 1.0$ equiv.) and benzene ( $1 \mathrm{~mL})$. The reaction mixture was stirred at $30{ }^{\circ} \mathrm{C}$ for 4 hours. The solvent was then removed by reduced pressure and the crude mixture was recrystallised in hot hexanes to yield $\mathbf{4 f}$ (526.1 $\mathrm{mg}, 2.28 \mathrm{mmol}, 99 \%)$ as a yellow powder.

Mp: $129-132{ }^{\circ} \mathrm{C}$ (pet. ether/ethyl actetate) (literature mp: $\left.123-125^{\circ} \mathrm{C}\right)^{5}$; $\mathrm{R}_{\mathrm{f}}: 0.2$ in $10: 1$ pet. ether:EtOAc; $v_{\max } / \mathrm{cm}^{-1}: 2960,2928,2864,1753,1743,1661,1442,1380,1115,1073,1034$; ${ }^{1} \mathrm{H}$ NMR (400 MHz, $\left.\mathrm{CDCl}_{3}\right) \delta 6.47$ (s, 2H, =CH), 2.93 (s, 2H, CH), $1.42\left(\mathrm{~s}, 6 \mathrm{H}, \mathrm{CH}_{3}\right), 1.40$ (s, $\left.6 \mathrm{H}, \mathrm{CH}_{3}\right), 1.31(\mathrm{~d}, J=8.3 \mathrm{~Hz}, 1 \mathrm{H}, \underline{\mathrm{CH}}), 1.26(\mathrm{~d}, J=8.3 \mathrm{~Hz}, 1 \mathrm{H}, \mathrm{CH} \underline{\mathrm{H}}) ;{ }^{13} \mathrm{C} \mathrm{NMR}(101 \mathrm{MHz}$, $\left.\mathrm{CDCl}_{3}\right) \delta 199.2(\mathrm{C}=\mathrm{O}), 141.7(\mathrm{CH}), 137.6(\mathrm{C}), 61.6(\mathrm{C}), 59.0\left(\mathrm{CH}_{2}\right), 57.1(\mathrm{CH}), 16.4\left(\mathrm{CH}_{3}\right)$, $11.1\left(\mathrm{CH}_{3}\right)$; HRMS (TOF MS ASAP+) $\mathrm{m} / z$ calc. for $\mathrm{C}_{15} \mathrm{H}_{19} \mathrm{O}_{2}: 231.1385[\mathrm{M}+\mathrm{H}]^{+}$found: 231.1381 . 


\section{1,2,3,4,9-Pentamethyl-1,4,4a,8a-tetrahydro-1,4-methan-naphthalene-5,8-dione ${ }^{10} \mathbf{~ g ~}$}

Synthesised according to modified literature procedure. ${ }^{10}$
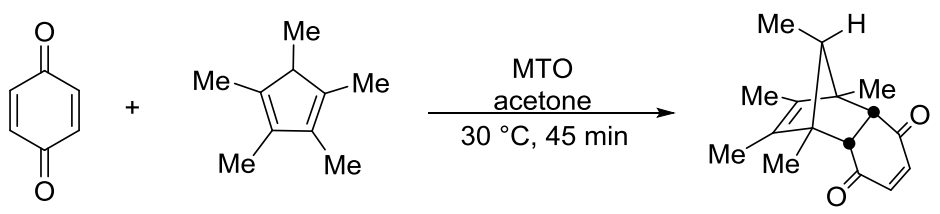

$4 \mathrm{~g}$

1,2,3,4,5-Pentamethylcyclopentadiene ( $391 \mu \mathrm{L}, 2.50 \mathrm{mmol}, 1.0$ equiv.) was added to a flask containing benzoquinone (270.9 mg, $2.50 \mathrm{mmol}, 1.0$ equiv.), methyltrioxorhenium (6.2 mg, $0.025 \mathrm{mmol}, 0.01$ equiv.) and acetone $(1 \mathrm{~mL})$. The reaction mixture was stirred at $30{ }^{\circ} \mathrm{C}$ for 45 min. The solvent was then removed by reduced pressure and the crude mixture was recrystallised in hot hexanes to yield $\mathbf{4 g}(600.9 \mathrm{mg}, 2.46 \mathrm{mmol}, 98 \%$, 6:1 d.r.), further purification was carried out by silica gel column chromatography (20:1 toluene:DCM $\rightarrow$ 10:1 toluene:DCM) to yield $\mathbf{4 g}$ (65.0 mg, $0.26 \mathrm{mmol}, 11 \%, 20: 1$ d.r.) as a yellow powder.

$\mathrm{Mp}=125-128^{\circ} \mathrm{C}($ Toluene/DCM $) ; \mathrm{Rf}=0.57$ in $3: 1$ pet. ether:EtOAc; $v_{\max } / \mathrm{cm}^{-1}: 2694,2927$, 2869, 1730, 1660. 1440, 1381, 1115, 873; ${ }^{1} \mathrm{H}$ NMR (300 MHz, $\left.\mathrm{CDCl}_{3}\right) \delta 6.44$ (s, 2H, =CH), $2.82(\mathrm{~s}, 2 \mathrm{H}, \mathrm{CH}), 1.41\left(\mathrm{q}, J=6.4 \mathrm{~Hz}, 1 \mathrm{H}, \mathrm{CHCH}_{3}\right), 1.34\left(\mathrm{~s}, 6 \mathrm{H}, \mathrm{CH}_{3}\right), 1.23\left(\mathrm{~s}, 6 \mathrm{H}, \mathrm{CH}_{3}\right), 0.58$ (d, $\left.J=6.4 \mathrm{~Hz}, 3 \mathrm{H}, \mathrm{CHC}_{3}\right) ;{ }^{13} \mathrm{C} \mathrm{NMR}\left(101 \mathrm{MHz}, \mathrm{CDCl}_{3}\right) \delta 199.2(\mathrm{C}), 141.9(\mathrm{CH}), 133.9(\mathrm{C})$, $62.8(\mathrm{CH}), 61.9(\mathrm{C}), 57.1(\mathrm{CH}), 14.1\left(\mathrm{CH}_{3}\right), 11.4\left(\mathrm{CH}_{3}\right), 7.5\left(\mathrm{CH}_{3}\right)$. 


\section{9,10-Dihydro-9,10-[1,2]benzenoanthracene-13,16-dionex ${ }^{11} 4 \mathrm{~h}$}

Synthesised according to adapted literature procedure. ${ }^{11}$
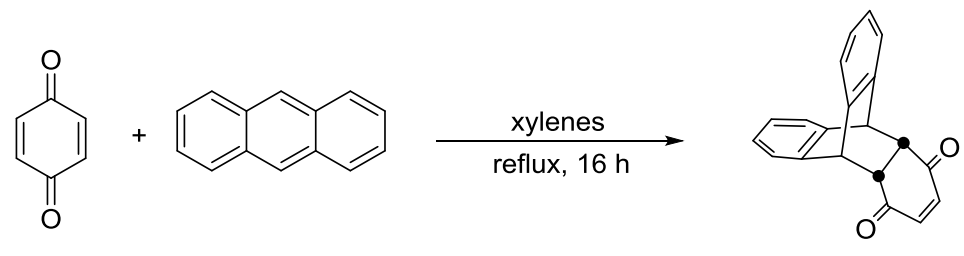

4h

To oven dried reflux apparatus, back-filled with argon, benzoquinone $(301.0 \mathrm{mg}, 2.50 \mathrm{mmol}$, 1.00 equiv.) was dissolved in xylenes $(5 \mathrm{~mL})$ and stirred for 5 minutes before anthracene was added to the reaction $(300.0 \mathrm{mg}, 2.80 \mathrm{mmol}, 1.10$ equiv.). The reaction was stirred at reflux for $16 \mathrm{~h}$. The reaction was cooled, and the crude product filtered and washed with cold xylenes. The crude product was recrystallised in hot xylenes to yield $\mathbf{4 h}(530.2 \mathrm{mg}, 1.86 \mathrm{mmol}, 74 \%)$ as a yellow powder.

Mp: $219-221{ }^{\circ} \mathrm{C}$ (xylenes) (literature mp: $231-233{ }^{\circ} \mathrm{C}$ ) ${ }^{11}$; $\mathrm{R}_{\mathrm{f}}: 0.36$ in 5:1 pet. ether:EtOAc; ${ }^{1} \mathrm{H}$ $\operatorname{NMR}\left(300 \mathrm{MHz}, \mathrm{CDCl}_{3}\right) \delta 7.43-7.36(\mathrm{~m}, 2 \mathrm{H}, \mathrm{Ar}-\mathrm{H}), 7.23-7.15(\mathrm{~m}, 4 \mathrm{H}, \mathrm{Ar}-\mathrm{H}), 7.11-7.05$ (m, 2H, Ar-H), 6.31 (s, 2H, =CH), 4.87 (app. t, $J=1.3 \mathrm{~Hz}, 2 \mathrm{H}$, CㅍAr), 3.14 (app. t, $J=1.3$ $\mathrm{Hz}, 2 \mathrm{H}, \mathrm{CH}) ;{ }^{13} \mathrm{C}$ NMR $\left(75 \mathrm{MHz}, \mathrm{CDCl}_{3}\right) \delta 198.5(\mathrm{C}), 141.7(\mathrm{C}), 140.7(\mathrm{CH}), 139.8(\mathrm{C}), 126.9$ $(\mathrm{CH}), 126.8(\mathrm{CH}), 124.9(\mathrm{CH}), 124.0(\mathrm{CH}), 49.2(\mathrm{CH}), 49.1(\mathrm{CH})$; HRMS (TOF MS ASAP+) $m / z$ calc. for $\mathrm{C}_{20} \mathrm{H}_{13} \mathrm{O}_{2}: 285.0916[\mathrm{M}-\mathrm{H}]^{+}$found: 285.0916 . 


\section{1,4,4a,8a-Tetrahydro-1,4-methanonaphthalene-5,8-dione ${ }^{12} 4 i$}

Synthesised according to adapted literature procedure. ${ }^{12}$

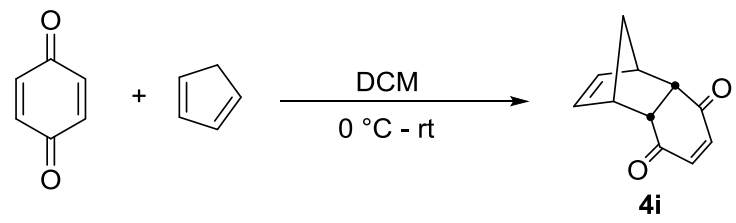

To an oven dried flask, back-filled with argon, benzoquinone (1.082 g, $10.00 \mathrm{mmol}, 1.00$ equiv.) was dissolved in DCM (5 mL) and stirred at $0{ }^{\circ} \mathrm{C}$. Cyclopentadiene $(0.85 \mathrm{~mL}, 10.40$ mmol, 1.04 equiv.) was added drop-wise over $1.5 \mathrm{~h}$ and the reaction was left to stir at $0{ }^{\circ} \mathrm{C}$ for $1 \mathrm{~h}$, and then warmed to room temperature and stirred for a further 30 minutes. The reaction was concentrated under reduced pressure. The crude mixture was recrystallised from hot hexane, left to cool on ice and filtered to yield $4 \mathbf{i}(1.54 \mathrm{~g}, 8.81 \mathrm{mmol}, 88 \%$ yield $)$ as pale-yellow crystals.

Mp: 53-54 ${ }^{\circ} \mathrm{C}$ (hexane) (literature mp: $\left.50-52{ }^{\circ} \mathrm{C}\right){ }^{13} \mathrm{R}_{\mathrm{f}}$ : 0.28 in 5:1 pet. ether/EtOAc; $v_{\max } / \mathrm{cm}^{-}$ 1: 3028, 2979, 2925, 1755, 1715, 1453, 1438, 1410, 1372, 1326, 1199, 1078, 995, 789, 758, 705; ${ }^{1} \mathrm{H}$ NMR (400 MHz, $\left.\mathrm{CDCl}_{3}\right) \delta 6.57$ (s, 2H, =CH), 6.07 (app. t, $J=1.8 \mathrm{~Hz}, 2 \mathrm{H},=\mathrm{CH}$ ), 3.57 $-3.53(\mathrm{~m}, 2 \mathrm{H}, \mathrm{CH}), 3.22(\mathrm{dd}, J=2.4,1.5 \mathrm{~Hz}, 2 \mathrm{H} \mathrm{CH}), 1.54(\mathrm{dt}, J=8.8,1.8 \mathrm{~Hz}, 1 \mathrm{H}, \mathrm{C} \underline{\mathrm{H}} \mathrm{H})$, $1.43(\mathrm{dm}, J=8.8 \mathrm{~Hz}, 1 \mathrm{H}, \mathrm{CH} \underline{\mathrm{H}}) ;{ }^{13} \mathrm{C} \mathrm{NMR}\left(75 \mathrm{MHz}, \mathrm{CDCl}_{3}\right) \delta 199.6(\mathrm{C}), 142.2(\mathrm{CH}), 135.4$ $(\mathrm{CH}), 48.9(\mathrm{CH}), 48.9\left(\mathrm{CH}_{2}\right), 48.5(\mathrm{CH})$. 
9,14-Diphenyl-9,9a,13a,14-tetrahydro-9,14-methanobenzo[f]tetraphene-10,13,15trione $^{14} \mathbf{4 j}$

Synthesised according to modified literature procedure. ${ }^{14}$
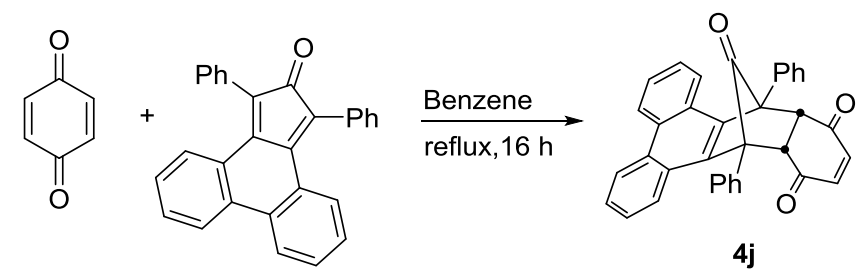

Phencyclone (290.0 mg, $0.5 \mathrm{mmol}, 1.0$ equiv.) was added reflux apparatus containing benzoquinone ( $80.0 \mathrm{mg}, 0.75 \mathrm{mmol}, 1.5$ equiv.) and benzene $(5 \mathrm{~mL})$ and stirred at reflux for $16 \mathrm{~h}$. The solvent was then removed under reduced pressure and the resulting crude was recrystallised with hot $\mathrm{CHCl}_{3}$ to yield $\mathbf{4 j}$ (215.4 $\mathrm{mg}, 0.44 \mathrm{mmol}, 88 \%$ yield) as a pale yellow powder.

Mp: decomp. $265-268{ }^{\circ} \mathrm{C}\left(\mathrm{CHCl}_{3}\right)$ (Literature: $\left.278-282{ }^{\circ} \mathrm{C}\right)^{14}$; $\mathrm{R}_{\mathrm{f}}$ : 0.1 in 5:1 pet. ether:EtOAc; vmax/ $\mathrm{cm}^{-1}: 3027,1787,1687,1603,1582,1567,1498,1447,750,726,698 ;{ }^{1} \mathrm{H}$ NMR (300 MHz, CDCl 3 ) $\delta 8.69$ (d, J=8.4 Hz, 2H, Ar-H), 8.29 (d, J=7.6 Hz, 2H, Ar-H), 7.67 (td, $J=7.6,1.5 \mathrm{~Hz}, 2 \mathrm{H}, \mathrm{Ar}-\mathrm{H}), 7.58$ (ddd, $J=8.4,6.8,1.5 \mathrm{~Hz}, 2 \mathrm{H}, \mathrm{Ar}-\mathrm{H}), 7.48$ (tt, $J=7.6,1.2$ $\mathrm{Hz}, 2 \mathrm{H}, \mathrm{Ar}-\mathrm{H}), 7.36$ (td, J=7.6, $1.5 \mathrm{~Hz}, 2 \mathrm{H}, \mathrm{Ar}-\mathrm{H}), 7.27$ (td, J=8.4, 1.2 Hz, 2H, Ar-H), 7.19 $(\mathrm{dd}, J=8.4,1.5 \mathrm{~Hz}, 2 \mathrm{H}, \mathrm{Ar}-\mathrm{H}), 7.09$ (dt, $J=8.0,1.5 \mathrm{~Hz}, 2 \mathrm{H}, \mathrm{Ar}-\mathrm{H}), 5.76(\mathrm{~s}, 2 \mathrm{H},=\mathrm{CH}), 4.62$ (s, 2H, CH); ${ }^{13} \mathrm{C}$ NMR (75 MHz, $\left.\mathrm{CDCl}_{3}\right) \delta 198.2(\mathrm{C}), 194.9(\mathrm{C}), 141.7(\mathrm{CH}), 134.2(\mathrm{C}), 133.4$ (C), $131.35(\mathrm{CH}), 131.32(\mathrm{C}), 129.1(\mathrm{CH}), 128.5(\mathrm{CH}), 128.35(\mathrm{CH}), 128.32(\mathrm{CH}), 127.3(\mathrm{CH})$, $126.9(\mathrm{CH}), 126.5(\mathrm{C}), 126.2(\mathrm{CH}), 123.4(\mathrm{CH}), 65.7(\mathrm{C}), 48.3(\mathrm{CH})$. 


\section{1,2,3,4-Tetrahydro-1,4-methanonaphthalene-5,8-dione $x$}
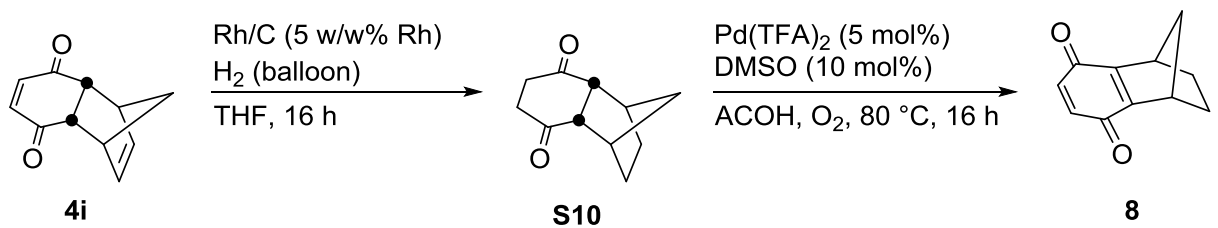

Diels-Alder adduct 4i (675.5 mg, $3.90 \mathrm{mmol}, 1.0$ equiv.) was added to a flask charged with $\mathrm{Rh} / \mathrm{C}$ (5 w/w\%, $58.8 \mathrm{mg}, 0.03 \mathrm{mmol}, 0.01$ equiv.) and THF (10 mL). The flask was sealed with a septum and $\mathrm{H}_{2}$ was backfilled into the flask via balloon (3 times). The reaction was stirred at room temperature under an atmosphere of $\mathrm{H}_{2}$ (balloon) for $16 \mathrm{~h}$. The reaction was filtered through a pad of celite to remove catalyst and washed with EtOAc $(3 \times 10 \mathrm{~mL})$. Solvent was removed under reduced pressure. The resulting crude mixture was purified by silica gel column chromatography (3:1 pet. ether:EtOAc) to yield $\mathbf{S 1 0}(348.5 \mathrm{mg}, 2.0 \mathrm{mmol}, 50 \%)$ as a colourless oil.

$\mathrm{R}_{\mathrm{f}}$ : 0.2 in $3: 1$ pet. ether:EtOAc; vmax $/ \mathrm{cm}^{-1}: 2991,2906,1701,1308,1152,932 ;{ }^{1} \mathrm{H}$ NMR (300 $\mathrm{MHz}, \mathrm{CDCl} 3) \delta=2.96-2.91(\mathrm{~m}, 2 \mathrm{H}, \mathrm{CH}), 2.86-2.82(\mathrm{~m}, 2 \mathrm{H}, \mathrm{Ar}-\mathrm{H}, \mathrm{CH}), 2.82-2.71(\mathrm{~m}$, $2 \mathrm{H}, \mathrm{C} \underline{\mathrm{H}}), 2.56-2.41$ (m, 2H, $\mathrm{CH} \underline{\mathrm{H}}), 1.58-1.46\left(\mathrm{~m}, 2 \mathrm{H}, \mathrm{CH}_{2}\right), 1.46-1.37\left(\mathrm{~m}, 2 \mathrm{H}, \mathrm{CH}_{2}\right)$, $1.36-1.25\left(\mathrm{~m}, 2 \mathrm{H}, \mathrm{CH}_{2}\right) ;{ }^{13} \mathrm{C} \mathrm{NMR}\left(75 \mathrm{MHz}, \mathrm{CDCl}_{3}\right) \delta=210.2(\mathrm{C}), 52.2(\mathrm{CH}), 41.7(\mathrm{CH})$, $39.2\left(\mathrm{CH}_{2}\right), 38.8\left(\mathrm{CH}_{2}\right), 24.7\left(\mathrm{CH}_{2}\right)$; HRMS (TOF MS EI +) $\mathrm{m} / z$ calc. for $\mathrm{C}_{11} \mathrm{H}_{12} \mathrm{O}_{2}: 176.0837$ $[\mathrm{M}-2 \mathrm{H}]^{+}$; found: 176.0839 .

S10 (53.9 mg, $0.3 \mathrm{mmol}, 1.0$ equiv.) was added to reflux apparatus containing $\operatorname{Pd}(\mathrm{TFA})_{2}(5.0$ $\mathrm{mg}, 0.015 \mathrm{mmol}, 0.05$ equiv.), DMSO (2.0 $\mu \mathrm{L}, 0.03 \mathrm{mmol}, 0.1$ equiv.) and glacial acetic acid $\left(1.5 \mathrm{~mL}\right.$ ). The reaction was stirred at $80{ }^{\circ} \mathrm{C}$ under an atmosphere of $\mathrm{O}_{2}$ (balloon) for $16 \mathrm{~h}$. Solvent was then removed under reduced pressure. The resulting crude was purified by silica gel column chromatography (10:1 pet. ether:EtOAc) to yield $8(44.0 \mathrm{mg}, 0.25 \mathrm{mmol}, 85 \%)$ as a yellow oil.

Rf: 0.2 in 10:1 pet. ether:EtOAc; vmax/ $\mathrm{cm}^{-1}: 2951,2876,1645,1578,1448,1323,944,834$; ${ }^{1} \mathrm{H}$ NMR $(300 \mathrm{MHz}, \mathrm{CDCl} 3) \delta=6.57(\mathrm{~s}, 2 \mathrm{H},=\mathrm{CH}), 3.48(\mathrm{dq}, J=3.0,1.7 \mathrm{~Hz}, 2 \mathrm{H}, \mathrm{CH}), 1.96-$ $1.88(\mathrm{dm}, J=12.1 \mathrm{~Hz}, 2 \mathrm{H}, \mathrm{C} \underline{\mathrm{HH}}), 1.63(\mathrm{dt}, J=9.1,1.7 \mathrm{~Hz}, 1 \mathrm{H}, \mathrm{C} \underline{H} \mathrm{H}), 1.40(\mathrm{dt}, J=9.1,1.4$ $\mathrm{Hz}, 1 \mathrm{H}, \mathrm{CH} \underline{\mathrm{H}}), 1.17$ (ddd, $J=12.1,4.2,2.3 \mathrm{~Hz}, 2 \mathrm{H}, \mathrm{CH} \underline{\mathrm{H}}),{ }^{13} \mathrm{C} \mathrm{NMR}\left(75 \mathrm{MHz}, \mathrm{CDCl}_{3}\right) \delta=$ $184.5(\mathrm{C}), 151.7(\mathrm{C}), 136.3(\mathrm{CH}), 47.9\left(\mathrm{CH}_{2}\right), 40.7(\mathrm{CH}), 25.1\left(\mathrm{CH}_{2}\right)$; HRMS (TOF MS ASAP +) $\mathrm{m} / z$ c calc. for $\mathrm{C}_{11} \mathrm{H}_{11} \mathrm{O}_{2}: 175.0759[\mathrm{M}-\mathrm{H}]^{+}$; found: 175.0759 . 


\section{Conjugate Addition Products}

\section{General Racemic Reaction Procedure}

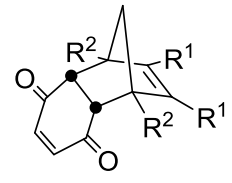

4<smiles>[12BH]B(O)O</smiles>

2

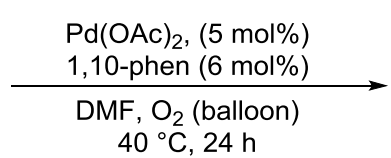

$40{ }^{\circ} \mathrm{C}, 24 \mathrm{~h}$

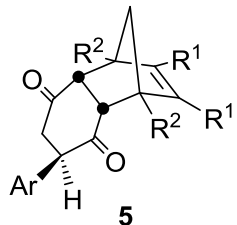

To a round-bottom flask, dried and back-filled with oxygen (via balloon), Diels-Alder adduct 4 (0.10 mmol, 1.0 equiv), boronic acid 2 (0.24 mmol, 2.4 equiv), $\mathrm{Pd}(\mathrm{OAc})_{2}(1.1 \mathrm{mg}, 0.005$ mmol, 0.05 equiv.) and 1,10-phenanthroline ( $1.1 \mathrm{mg}, 0.006 \mathrm{mmol}, 0.05$ equiv.) were stirred in DMF (1.0 mL) at $40{ }^{\circ} \mathrm{C}$ under an atmosphere of oxygen (balloon) for $24 \mathrm{~h}$. The reaction was then diluted with 2:1 Et $2 \mathrm{O}$ :EtOAc $(15 \mathrm{~mL})$ and the organic layer was washed with $\mathrm{H}_{2} \mathrm{O}(3 \times 10$ $\mathrm{mL})$ and brine $(1 \times 10 \mathrm{~mL})$, the combined organic layers were dried over $\mathrm{MgSO}_{4}$ and solvent was removed under reduced pressure. The resulting crude was purified by silica gel column chromatography and separating conditions were determined by chiral stationary phase HPLC.

\section{General Enantioselective Reaction Procedure A}

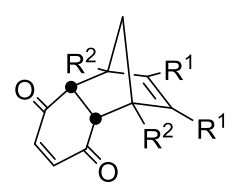

4<smiles>[12BH]B(O)[17OH]</smiles>

2

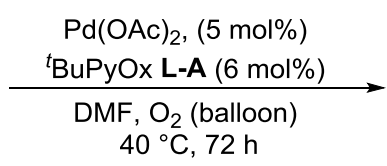
$40^{\circ} \mathrm{C}, 72 \mathrm{~h}$

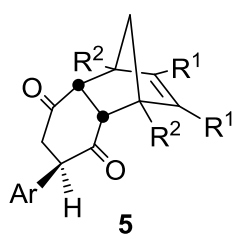

To a round-bottom flask, dried and back-filled with oxygen (via balloon), $\mathrm{Pd}(\mathrm{OAc})_{2}(1.1 \mathrm{mg}$, 0.005, 0.05 equiv.) and ${ }^{t} \mathrm{BuPyOx} \mathbf{L}-\mathbf{A}(1.2 \mathrm{mg}, 0.006 \mathrm{mmol}, 0.06$ equiv.) were premixed in DMF (0.3 mL) for approx. 30 minutes at room temperature. Diels-Alder adduct 4 (0.10 mmol, 1.0 equiv) and boronic acid 2 ( 0.24 mmol, 2.4 equiv) were then added, along with DMF $(0.7$ $\mathrm{mL}$ ) and the reaction was stirred at $40{ }^{\circ} \mathrm{C}$ under an atmosphere of oxygen (balloon) for $72 \mathrm{~h}$. The reaction was then diluted with 2:1 Et $\mathrm{t}_{2} \mathrm{O}: \operatorname{EtOAc}(15 \mathrm{~mL})$ and the organic layer was washed with $\mathrm{H}_{2} \mathrm{O}(3 \times 10 \mathrm{~mL})$ and brine $(1 \times 10 \mathrm{~mL})$, the combined organic layers were dried over $\mathrm{MgSO}_{4}$ and solvent was removed under reduced pressure. The resulting crude was purified by silica gel column chromatography and enantiomeric ratios were determined by chiral stationary phase HPLC.

\section{General Enantioselective Reaction Procedure B}

In instances where the product and starting material had the same $R_{f}$ value, the reaction was carried out under an increased ligand and catalyst loading to ensure the reaction went to completion (so that an isolated yield could be obtained). 


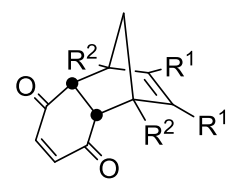

4

$$
\int_{\mathrm{Ar}}^{\mathrm{HO}^{-}}{ }^{-\mathrm{OH}}
$$

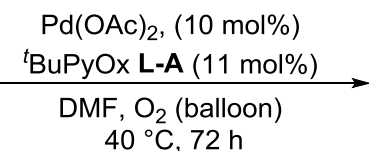

$40{ }^{\circ} \mathrm{C}, 72 \mathrm{~h}$

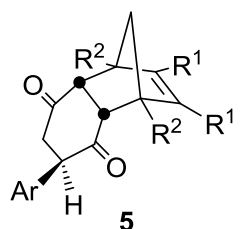

To a round-bottom flask, dried and back-filled with oxygen (via balloon), $\mathrm{Pd}(\mathrm{OAc})_{2}(2.2 \mathrm{mg}$, $0.010,0.10$ equiv.) and ${ }^{t} \mathrm{BuPyOx} \mathbf{L}-\mathbf{A}(2.2 \mathrm{mg}, 0.011 \mathrm{mmol}, 0.11$ equiv.) were premixed in DMF (0.3 mL) for approx. 30 minutes at room temperature. Diels-Alder adduct $4(35.4 \mathrm{mg}$, 0.0999 mmol, 1.0 equiv) and boronic acid 2 (0.24 mmol, 2.4 equiv) were then added, along with DMF $(0.7 \mathrm{~mL})$ and the reaction was stirred at $40{ }^{\circ} \mathrm{C}$ under an atmosphere of oxygen (balloon) for $72 \mathrm{~h}$. The reaction was then diluted with 2:1 $\mathrm{Et}_{2} \mathrm{O}: \mathrm{EtOAc}(15 \mathrm{~mL})$ and the organic layer was washed with $\mathrm{H}_{2} \mathrm{O}(3 \times 10 \mathrm{~mL})$ and brine $(1 \times 10 \mathrm{~mL})$, the combined organic layers were dried over $\mathrm{MgSO}_{4}$ and solvent was removed under reduced pressure. The resulting crude was purified by silica gel column chromatography and enantiomeric ratios were determined by chiral stationary phase HPLC.

\section{General enantioselective procedure $\mathbf{C}$}

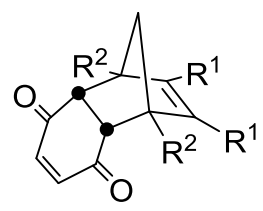

4<smiles>OB(O)[Al]</smiles>

2
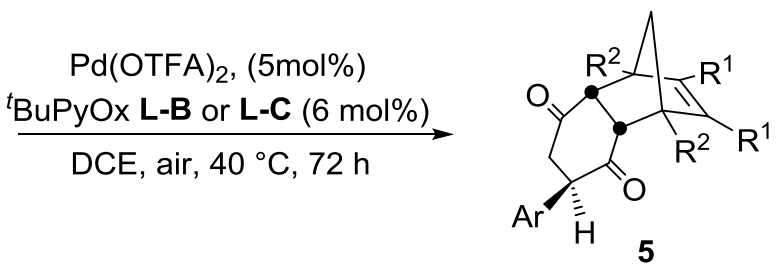

To an oven dried $4 \mathrm{~mL}$ vial, $\mathrm{Pd}(\mathrm{OTFA})_{2}(1.7 \mathrm{mg}, 0.005,0.05$ equiv. $)$ and $4 / 5-\mathrm{CF}_{3}{ }^{t} \mathrm{BuPyOx} \mathbf{L}-$ B or L-C $(0.006 \mathrm{mmol}, 0.06$ equiv. $)$ were pre-mixed in DCE $(0.3 \mathrm{~mL})$ for approx. 30 minutes at room temperature. Diels-Alder adduct $4(0.10 \mathrm{mmol}, 1.0$ equiv) and boronic acid $2(0.24$ mmol, 2.4 equiv) were then added, along with DCE $(0.2 \mathrm{~mL})$, the vial was sealed and the reaction was stirred at $40{ }^{\circ} \mathrm{C}$ for $72 \mathrm{~h}$. The reaction was then filtered through a silica plug in a Pasteur pipette using DCM (15 mL) and solvent was then removed under reduced pressure. The resulting crude was purified by silica gel column chromatography and enantiomeric ratios were determined by chiral stationary phase HPLC. 
Diels-Alder Adduct Scope

\section{1,2,3,4-Tetrachloro-9,9-dimethoxy-6-(4-methoxyphenyl)-hexahydro-1,4- methanonaphthalene-5,8-dione 5a}

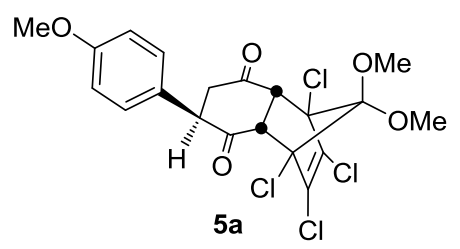

\section{Racemic procedure:}

General racemic procedure was followed with modifications, reacting $p$ methoxyphenylboronic acid 2a (37.0 mg, $0.25 \mathrm{mmol}, 2.5$ equiv.), Diels-Alder Adduct 4a (37.0 $\mathrm{mg}, 0.099 \mathrm{mmol}, 1.0$ equiv.), $\mathrm{Pd}(\mathrm{OAc})_{2}(1.1 \mathrm{mg}, 0.005 \mathrm{mmol}, 0.01$ equiv.) and 1,10phenanthroline (1.1 mg, $0.006 \mathrm{mmol}, 0.012$ equiv.) in DMF (1.0 mL) under at an atmosphere of $\mathrm{O}_{2}$ at $30{ }^{\circ} \mathrm{C}$ for $24 \mathrm{~h}$. The resulting crude was purified by silica gel column chromatography (hexane/EtOAc 5:1) to yield 5a (35.0 mg, $0.074 \mathrm{mmol}, 74 \%,>20: 1)$ as a white crystalline solid.

\section{Enantioselective procedure:}

General enantioselective procedure A was followed. Reacting $p$-methoxyphenylboronic acid 2a (37.3 mg, 0.25 mmol, 2.5 equiv.), Diels-Alder adduct 4a (39.6 mg, 0.099 mmol, 1.0 equiv.), $\mathrm{Pd}(\mathrm{OAc})_{2}\left(1.1 \mathrm{mg}, 0.005 \mathrm{mmol}, 0.01\right.$ equiv.) and ${ }^{t} \mathrm{BuPyOx} \mathbf{L}-\mathbf{A}$ (1.2 mg, $0.006 \mathrm{mmol}, 0.012$ equiv.) in DMF (1.0 mL) under at an atmosphere of $\mathrm{O}_{2}$ at $30{ }^{\circ} \mathrm{C}$ for $72 \mathrm{~h}$. The resulting crude was purified by silica gel column chromatography (hexanes/EtOAc 5:1) to yield 5a $(29.9 \mathrm{mg}$, $0.063 \mathrm{mmol}, 80 \%,>20: 1$ d.r., $92: 8$ e.r.) as an off-white crystalline solid.

Mp: $139-141^{\circ} \mathrm{C}$ (hexanes/chloroform); $\mathrm{R}_{\mathrm{f}}: 0.28$ in 3:1 pet. ether:EtOAc; vmax/ $\mathrm{cm}^{-1}: 2956$, $2863,1716,1612,1596,1514,1250,1189,1120,844,808,720 ;{ }^{1} \mathrm{H}$ NMR $\left(300 \mathrm{MHz}, \mathrm{CDCl}_{3}\right)$ $7.00-6.93(\mathrm{~d}, J=9.0 \mathrm{~Hz}, 2 \mathrm{H}, \mathrm{Ar}-\mathrm{H}), 6.82(\mathrm{~d}, J=9.0 \mathrm{~Hz}, 2 \mathrm{H}, \mathrm{Ar}-\mathrm{H}), 3.73\left(\mathrm{~s}, 3 \mathrm{H}, \mathrm{OCH}_{3}\right), 3.65$ (dd, $J=9.0,4.9 \mathrm{~Hz}, 1 \mathrm{H}, \mathrm{C} \underline{H} A r), 3.60(\mathrm{~d}, J=10.0 \mathrm{~Hz}, 1 \mathrm{H}, \mathrm{CH}), 3.51$ (s, 3H, OCH$), 3.51$ (s, $\left.3 \mathrm{H}, \mathrm{OCH}_{3}\right), 3.48(\mathrm{~d}, 10.0 \mathrm{~Hz}, 1 \mathrm{H}, \mathrm{CH}), 3.05(\mathrm{dd}, J=16.0,9.0 \mathrm{~Hz}, 1 \mathrm{H}, \mathrm{C} \underline{\mathrm{HH}}), 2.65$ (dd, $J=$ 16.0, 4.9 Hz, 1H, CH프); $\delta{ }^{13} \mathrm{C} \mathrm{NMR}\left(101 \mathrm{MHz}, \mathrm{CDCl}_{3}\right) 202.2(\mathrm{C}), 202.1$ (C), 159.6 (C), 130.8 (C), $130.5(\mathrm{C}), 128.9(\mathrm{CH}), 126.4(\mathrm{C}), 114.8(\mathrm{CH}), 111.5(\mathrm{C}), 76.58(\mathrm{C}), 76.55(\mathrm{C}), 57.0(\mathrm{CH})$, $56.3(\mathrm{CH}), 55.5\left(\mathrm{CH}_{3}\right), 53.4\left(\mathrm{CH}_{3}\right), 52.3\left(\mathrm{CH}_{3}\right), 52.2(\mathrm{CH}), 43.8\left(\mathrm{CH}_{2}\right)$. HRMS (TOF MS ASAP +) $m / z$ calc. for $\mathrm{C}_{20} \mathrm{H}_{19} \mathrm{O}_{5} \mathrm{Cl}_{4}: 478.9987[\mathrm{M}+\mathrm{H}]^{+}$; found: $478.9984 ; \quad[\alpha]_{\mathrm{D}}^{21.0}=+4.8(\mathrm{c}$ $0.5, \mathrm{CHCl}_{3}$ ); 92:8 e.r.; HPLC (CHIRALPAK IB, hexane/2-propanol: 85:15, flow rate: $1.0 \mathrm{~mL}$ $\min ^{-1}$, detection UV $205 \mathrm{~nm}, 25^{\circ} \mathrm{C}$ ) $\mathrm{t}_{\mathrm{R}}$ of major isomer: $13.43 \mathrm{~min}, \mathrm{t}_{\mathrm{R}}$ of minor isomer: 21.35 $\min$. 


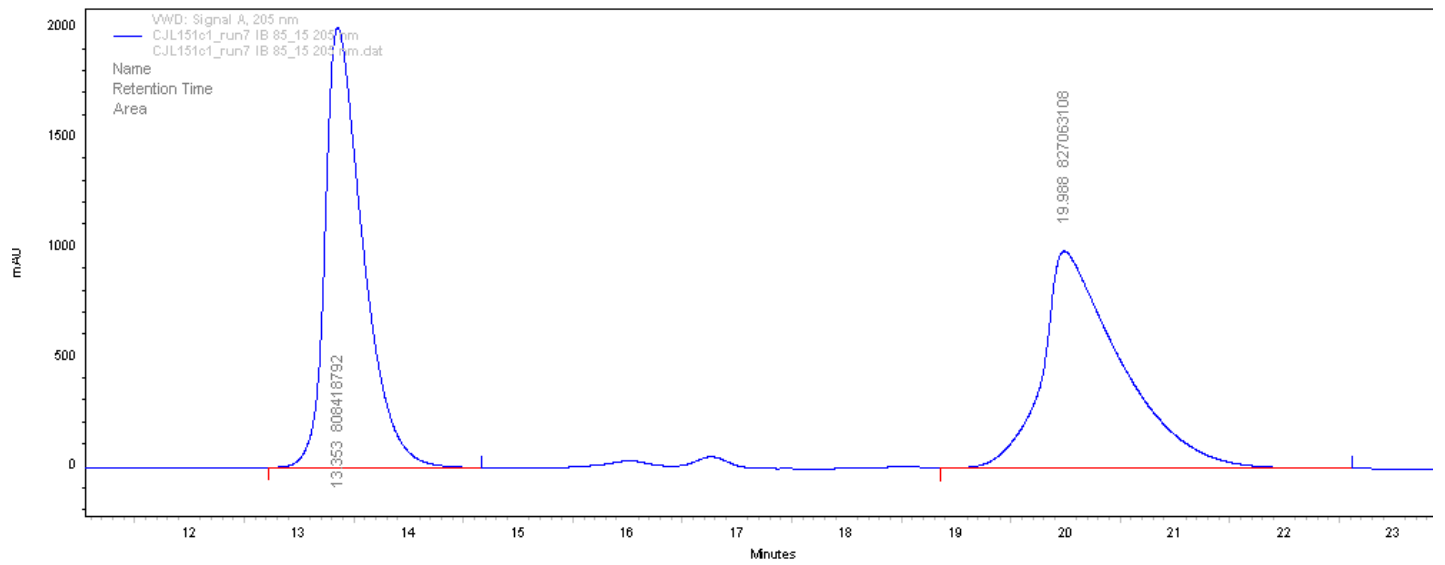

VWD : Signal A,

$205 \mathrm{~nm}$ Results

\begin{tabular}{rrrrr} 
Retention Time & Area & Area \% & Height & Height \% \\
\hline 13.353 & 808418792 & 49.43 & 33660470 & 66.94 \\
19.988 & 827063108 & 50.57 & 16621854 & 33.06
\end{tabular}

\begin{tabular}{|r|r|r|r|r|}
\hline Totals & 1635481900 & 100.00 & 50282324 & 100.00 \\
\hline
\end{tabular}

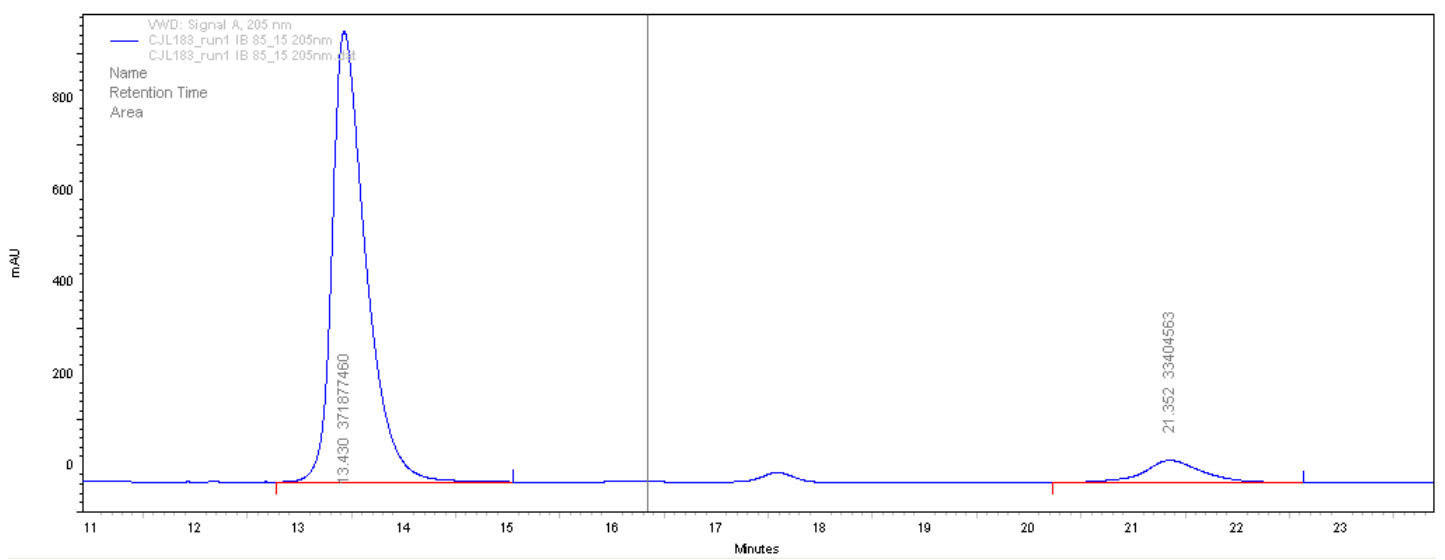

VWD : Signal A,

$205 \mathrm{~nm}$ Results

Retention Time

$\begin{array}{rrrrr}13.430 & 371877460 & 91.76 & 16487966 & 95.25 \\ 21.352 & 33404563 & 8.24 & 821618 & 4.75\end{array}$

Totals 


\section{1,2,3,4-Tetrachloro-6-(4-hydroxyphenyl)-9,9-dimethoxy-1,4,4a,6,7,8a-hexahydro-1,4- methanonaphthalene-5,8-dione 5a'}

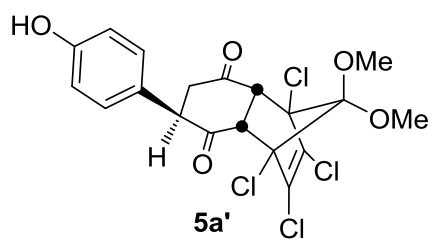

\section{Racemic procedure:}

General racemic procedure was followed with modifications, reacting $p$ methoxyphenylboronic acid 2m (33.1 mg, 0.24 mmol, 2.4 equiv.), Diels-Alder Adduct 4a (37.0 $\mathrm{mg}, 0.099 \mathrm{mmol}, 1.0$ equiv. $), \operatorname{Pd}(\mathrm{OAc})_{2}(1.1 \mathrm{mg}, 0.005 \mathrm{mmol}, 0.01$ equiv. $)$ and 1,10phenanthroline ( $1.1 \mathrm{mg}, 0.006 \mathrm{mmol}, 0.012$ equiv.) in DMF $(1.0 \mathrm{~mL})$ under at an atmosphere of $\mathrm{O}_{2}$ at $40{ }^{\circ} \mathrm{C}$ for $24 \mathrm{~h}$. The resulting crude was purified by silica gel column chromatography (hexane/EtOAc 20:1 $\rightarrow$ 3:1) to yield 5a' $(38.7 \mathrm{mg}, 0.083 \mathrm{mmol}, 83 \%,>20: 1)$ as a white crystalline solid.

\section{Enantioselective procedure:}

General enantioselective procedure A was followed. Reacting $p$-methoxyphenylboronic acid 2m (33.1 mg, 0.24 mmol, 2.4 equiv.), Diels-Alder adduct 4a (39.6 mg, 0.099 mmol, 1.0 equiv.), $\mathrm{Pd}(\mathrm{OAc})_{2}\left(1.1 \mathrm{mg}, 0.005 \mathrm{mmol}, 0.01\right.$ equiv.) and ${ }^{t} \mathrm{BuPyOx} \mathbf{L}-\mathbf{A}$ (1.2 mg, $0.006 \mathrm{mmol}, 0.012$ equiv.) in DMF (1.0 mL) under at an atmosphere of $\mathrm{O}_{2}$ at $40{ }^{\circ} \mathrm{C}$ for $72 \mathrm{~h}$. The resulting crude was purified by silica gel column chromatography (hexanes/EtOAc 5:1 $\rightarrow 3: 1$ ) to yield 5a' (30.0 mg, $0.065 \mathrm{mmol}, 65 \%$, >20:1 d.r., 90:10 e.r.) as an off-white crystalline solid.

Mp: $191-192{ }^{\circ} \mathrm{C}$ (hexanes/chloroform); $\mathrm{R}_{\mathrm{f}}: 0.2$ in 3:1 pet. ether:EtOAc; vmax/ $\mathrm{cm}^{-1}: 3455$, 2955, 1720, 1705, 1614, 1596, 518, 1439, 1188, 1126, 847, 808, 751; ${ }^{1} \mathrm{H}$ NMR (300 MHz, $\left.\mathrm{CDCl}_{3}\right) ; \delta 6.99(\mathrm{~d}, J=8.7 \mathrm{~Hz}, 2 \mathrm{H}, \mathrm{Ar}-\mathrm{H}), 6.82(\mathrm{~d}, J=8.7 \mathrm{~Hz}, 1 \mathrm{H}, \mathrm{Ar}-\mathrm{H}), 4.82(\mathrm{~s}, 1 \mathrm{H}, \mathrm{OH})$, $3.71(\mathrm{dd}, J=9.2,4.8 \mathrm{~Hz}, 1 \mathrm{H}, \mathrm{C} \underline{\mathrm{H} A r}), 3.67(\mathrm{~d}, J=9.9 \mathrm{~Hz}, 1 \mathrm{H}, \mathrm{CH}), 3.59$ (s, 3H, $\left.\mathrm{OCH}_{3}\right), 3.58$ (s, 3H, $\left.\mathrm{OCH}_{3}\right), 3.56$ (d, $\left.J=9.9 \mathrm{~Hz}, 1 \mathrm{H}, \mathrm{CH}\right), 3.11$ (dd, $\left.J=16.0,9.2 \mathrm{~Hz}, 1 \mathrm{H}, \mathrm{C} \underline{\mathrm{HH}}\right), 2.73$ (dd, $J=16.0,4.8 \mathrm{~Hz}, 1 \mathrm{H}, \mathrm{CH} \underline{\mathrm{H}}) ;{ }^{13} \mathrm{C} \mathrm{NMR}\left(101 \mathrm{MHz}, \mathrm{CDCl}_{3}\right) 202.2$ (C), 202.1 (C), $177.4(\mathrm{C})$, $155.6(\mathrm{C}), 130.8(\mathrm{C}), 130.6(\mathrm{C}), 129.1(\mathrm{CH}), 126.7(\mathrm{C}), 116.3(\mathrm{CH}), 111.6(\mathrm{C}), 76.6(\mathrm{C}), 57.1$ $(\mathrm{CH}), 56.3(\mathrm{CH}), 53.4\left(\mathrm{CH}_{3}\right), 52.3\left(\mathrm{CH}_{3}\right), 52.2(\mathrm{CH}), 43.9\left(\mathrm{CH}_{2}\right)$; HRMS (TOF MS ASAP +) $m / z$ calc. for $\mathrm{C}_{19} \mathrm{H}_{17} \mathrm{O}_{5} \mathrm{Cl}_{4}: 464.9830[\mathrm{M}+\mathrm{H}]^{+}$; found: $464.9825 ;[\alpha]_{\mathrm{D}}^{20.7}=+26.4($ c 1.67 , $\mathrm{CHCl}_{3}$ ); 90:10 e.r.; HPLC (CHIRALPAK IB, hexane/2-propanol: 85:15, flow rate: $1.0 \mathrm{~mL} \mathrm{~min}{ }^{-}$ ${ }^{1}$, detection UV $205 \mathrm{~nm}, 25^{\circ} \mathrm{C}$ ) $\mathrm{t}_{\mathrm{R}}$ of major isomer: $17.84 \mathrm{~min}, \mathrm{t}_{\mathrm{R}}$ of minor isomer: $25.64 \mathrm{~min}$. 


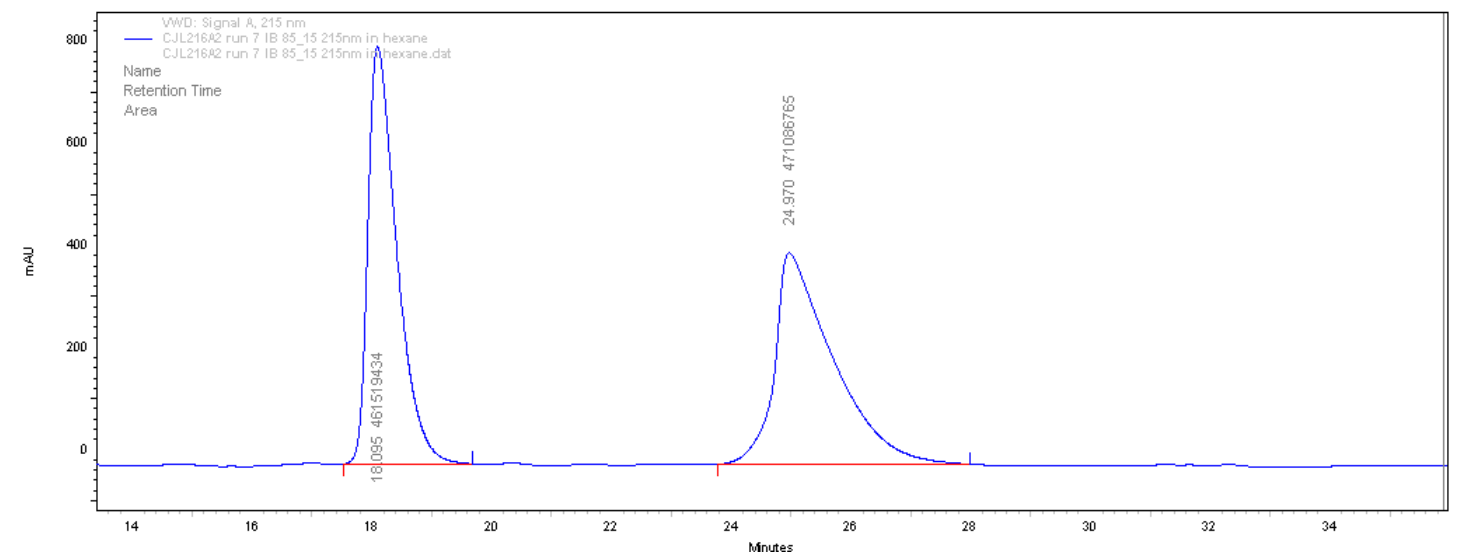

VWD: Signal A,

215 Ium Results

\begin{tabular}{rrrrr} 
Retention Time & Area & Area \% & Height & Height \% \\
\hline 18.095 & 461519434 & 49.49 & 13715473 & 66.39 \\
24.970 & 471086765 & 50.51 & 6942893 & 33.61
\end{tabular}

\begin{tabular}{|r|r|r|r|r|}
\hline Totals & 932606199 & 100.00 & 20658366 & 100.00 \\
\hline
\end{tabular}

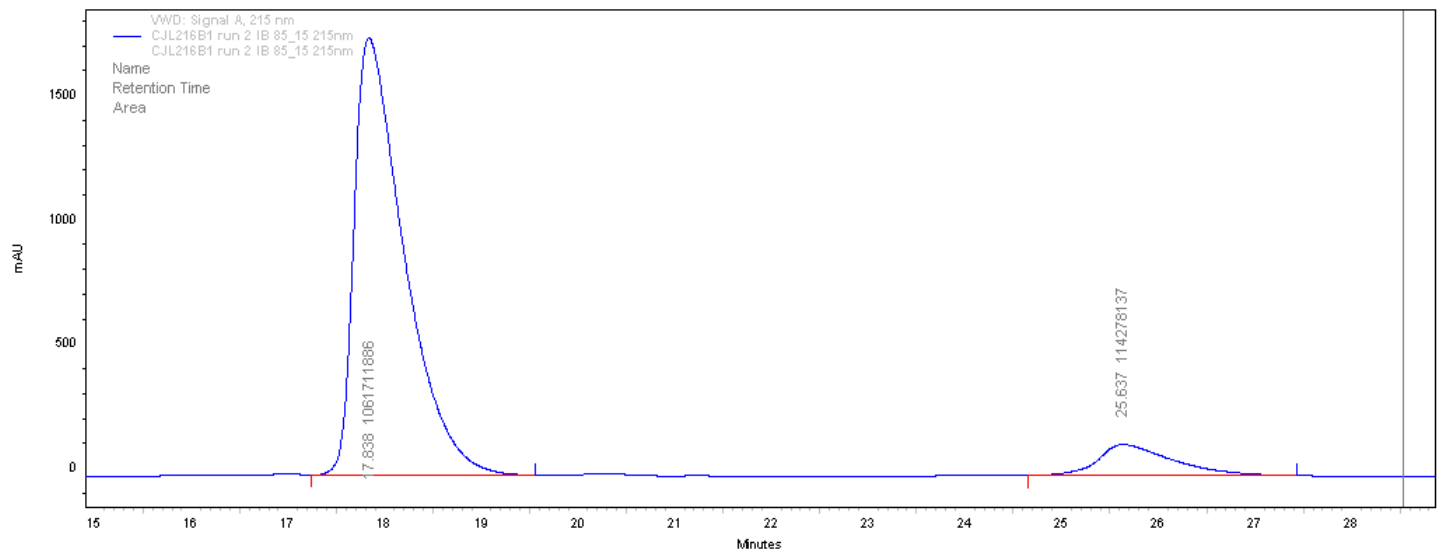

VWD: Signal A,

215 um Results

Retention Time

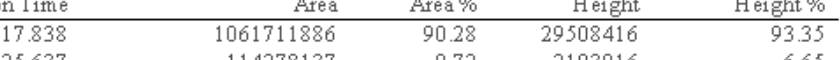

25.637

114278137

$9.72 \quad 2103016$

\begin{tabular}{|r|r|r|r|r|}
\hline Totals & 1175990023 & 100.00 & 31611432 & 100.00 \\
\hline
\end{tabular}




\section{6-(4-Methoxyphenyl)-1,4-dimethyl-2,3-diphenyl-1,4,4a,6,7,8a-hexahydro-1,4- methanonaphthalene-5,8-dione 5b}

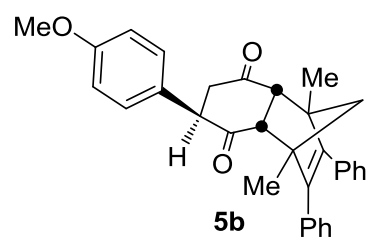

\section{Racemic procedure:}

General racemic procedure was followed. Reacting $p$-methoxybenzene boronic acid $\mathbf{2 b}$ (36.5 mg, 0.24 mmol, 2.4 equiv.), Diels-Alder adduct $4 \mathbf{b}$ (35.4 mg, 0.099 mmol, 1 equiv.), $\mathrm{Pd}(\mathrm{OAc})_{2}$ ( $1.1 \mathrm{mg}, 0.005 \mathrm{mmol}, 0.05$ equiv.) and 1,10-phenanthroline (1.1 mg, $0.006 \mathrm{mmol}, 0.06$ equiv.) in DMF $(1 \mathrm{~mL})$ under an atmospshere of $\mathrm{O}_{2}$ (balloon) at $40{ }^{\circ} \mathrm{C}$ for $24 \mathrm{~h}$.. The resulting crude was purified by silica gel column chromatography (20:1 toluene/EtOAc) to yield $\mathbf{5 b}$ (39.3 $\mathrm{mg}$, $0.085 \mathrm{mmol}, 85 \%,>20: 1$ d.r.) as a colourless crystalline solid.

\section{Enantioselective procedure:}

General enantioselective procedure A was followed. Reacting $p$-methoxylbenzene boronic acid 2a (36.5 mg, 0.24 mmol, 2.4 equiv.) with Diels-Alder adduct 4b (35.4 mg, 0.099 mmol, 1 equiv.) $\mathrm{Pd}(\mathrm{OAc})_{2}\left(1.1 \mathrm{mg}, 0.005 \mathrm{mmol}, 0.05\right.$ equiv.) and ${ }^{t} \mathrm{BuPyOx} \mathbf{L}-\mathbf{A}(1.2 \mathrm{mg}, 0.006 \mathrm{mmol}$, 0.06 equiv.) in DMF ( $1 \mathrm{~mL}$ ) under an atmosphere of $\mathrm{O}_{2}$ (balloon) at $40{ }^{\circ} \mathrm{C}$ for $72 \mathrm{~h}$. . The resulting crude was purified by silica gel column chromatography $(10: 1 \rightarrow 3: 1$ pet. ether/EtOAc) to yield $\mathbf{5 b}(32.3 \mathrm{mg}, 0.070 \mathrm{mmol}, 70 \%,>20: 1$ d.r., $97: 3$ e.r. $)$ as a colourless crystalline solid.

Mp: $158{ }^{\circ} \mathrm{C}$ (DCM/petrol ether); $\mathrm{R}_{\mathrm{f}}: 0.3$ in 5:1 pet. ether:EtOAc; $v_{\max } / \mathrm{cm}^{-1}: 2960,1932,1699$, 1611, 1514, 1471, 1248, 823, 743, 696; ${ }^{1} \mathrm{H}$ NMR (300 MHz, $\left.\mathrm{CDCl}_{3}\right) \delta 7.24-7.11$ (m, 6H, ArH), $7.04-6.83(\mathrm{~m}, 8 \mathrm{H}, \mathrm{Ar}-\mathrm{H}), 3.88(\mathrm{dd}, J=10.2,5.1 \mathrm{~Hz}, 1 \mathrm{H}, \mathrm{CHAr}), 3.79$ (s, 3H, OMe), 3.28 $(\mathrm{d}, J=9.9 \mathrm{~Hz}, 1 \mathrm{H}, \mathrm{CH}), 3.19(\mathrm{~d}, J=9.9 \mathrm{~Hz}, 1 \mathrm{H}, \mathrm{CH}), 3.06$ (dd, $J=16.0,10.1 \mathrm{~Hz}, 1 \mathrm{H}, \mathrm{C} \underline{\mathrm{H}})$, $2.80(\mathrm{dd}, J=16.0,5.1 \mathrm{~Hz}, 1 \mathrm{H}, \mathrm{CH} \underline{\mathrm{H}}), 1.83(\mathrm{~d}, J=8.5 \mathrm{~Hz}, 1 \mathrm{H}, \mathrm{CHH}), 1.62\left(\mathrm{~s}, 3 \mathrm{H}, \mathrm{CH}_{3}\right), 1.58$ $(\mathrm{d}, J=8.5 \mathrm{~Hz}, 1 \mathrm{H}, \mathrm{CH} \underline{\mathrm{H}}), 1.54\left(\mathrm{~s}, 3 \mathrm{H}, \mathrm{CH}_{3}\right) ;{ }^{13} \mathrm{C} \mathrm{NMR}\left(100 \mathrm{MHz}, \mathrm{CDCl}_{3}\right) \delta 208.68(\mathrm{C}), 208.66$ (C), 159.2 (C), $147.4(2 \times C), 145.0(\mathrm{C}), 135.7$ (C), 135.5 (C), $129.4(\mathrm{CH}), 129.3(\mathrm{CH}), 129.2$ $(\mathrm{CH}), 128.3(\mathrm{CH}), 128.1(\mathrm{CH}), 127.1(\mathrm{CH}), 114.5(\mathrm{CH}), 66.2\left(\mathrm{CH}_{2}\right), 60.2(\mathrm{CH}), 59.0(\mathrm{CH})$, $58.13(\mathrm{C}), 58.10(\mathrm{C}), 55.4\left(\mathrm{CH}_{3}\right), 52.2(\mathrm{CH}), 45.4\left(\mathrm{CH}_{2}\right), 19.1\left(\mathrm{CH}_{3}\right), 18.8\left(\mathrm{CH}_{3}\right)$ with 1 overlapping aromatic $\mathrm{CH}$ signal; HRMS (FTMS + p NSI) $\mathrm{m} / z$ calc. for $\mathrm{C}_{32} \mathrm{H}_{34} \mathrm{O}_{3} \mathrm{~N}$ : 480.2533 $\left[\mathrm{M}+\mathrm{NH}_{4}\right]^{+}$; found:480.2526; $[\alpha]_{\mathrm{D}}^{20.1}=+12.0\left(c 0.5, \mathrm{CHCl}_{3}\right)$; $97: 3$ e.r.; HPLC $($ CHIRALPAK 
IA, hexane/2-propanol: 97:3, flow rate: $1.0 \mathrm{~mL} \mathrm{~min}^{-1}$, detection UV $215 \mathrm{~nm}, 25^{\circ} \mathrm{C}$ ) $\mathrm{t}_{\mathrm{R}}$ of major isomer: $18.32 \mathrm{~min}, \mathrm{t}_{\mathrm{R}}$ of minor isomer: $19.99 \mathrm{~min}$.

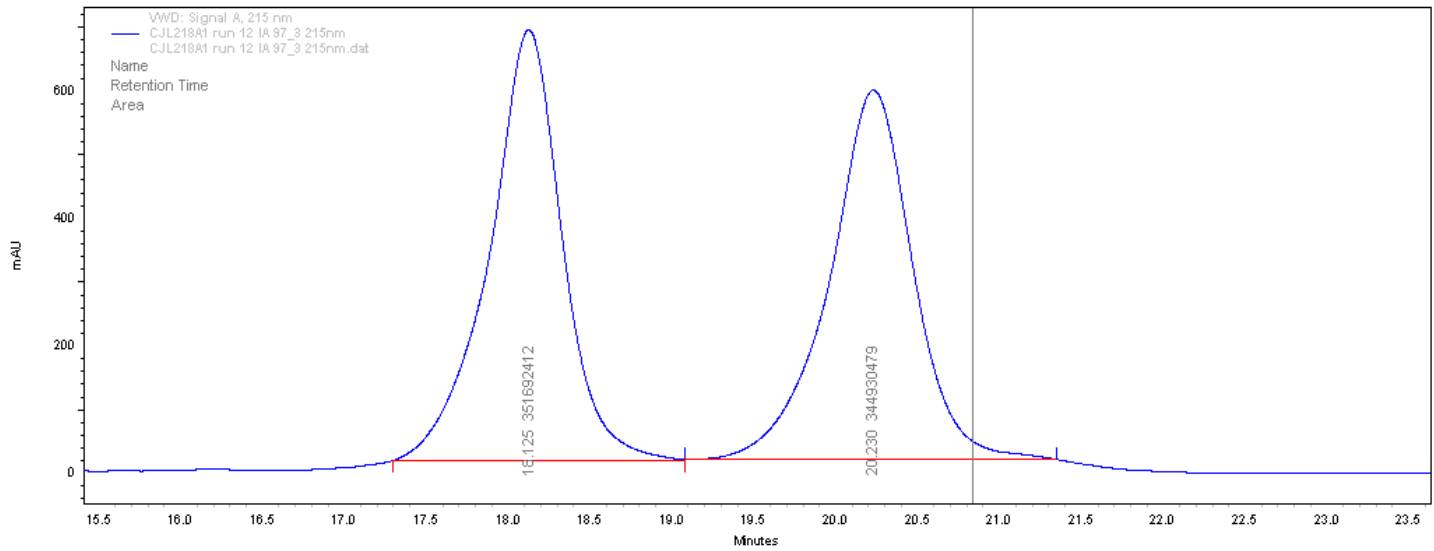

VWD: Signal A,

215 um Results

Retention Time

\begin{tabular}{rrrrr} 
Area & Area\% & Height & Height \% \\
\hline 18.125 & 351692412 & 50.49 & 11332086 & 53.83 \\
20.230 & 344930479 & 49.51 & 9719160 & 46.17
\end{tabular}

\begin{tabular}{|r|r|r|r|r|}
\hline Totals & 696622891 & 100.00 & 21051246 & 100.00 \\
\hline
\end{tabular}

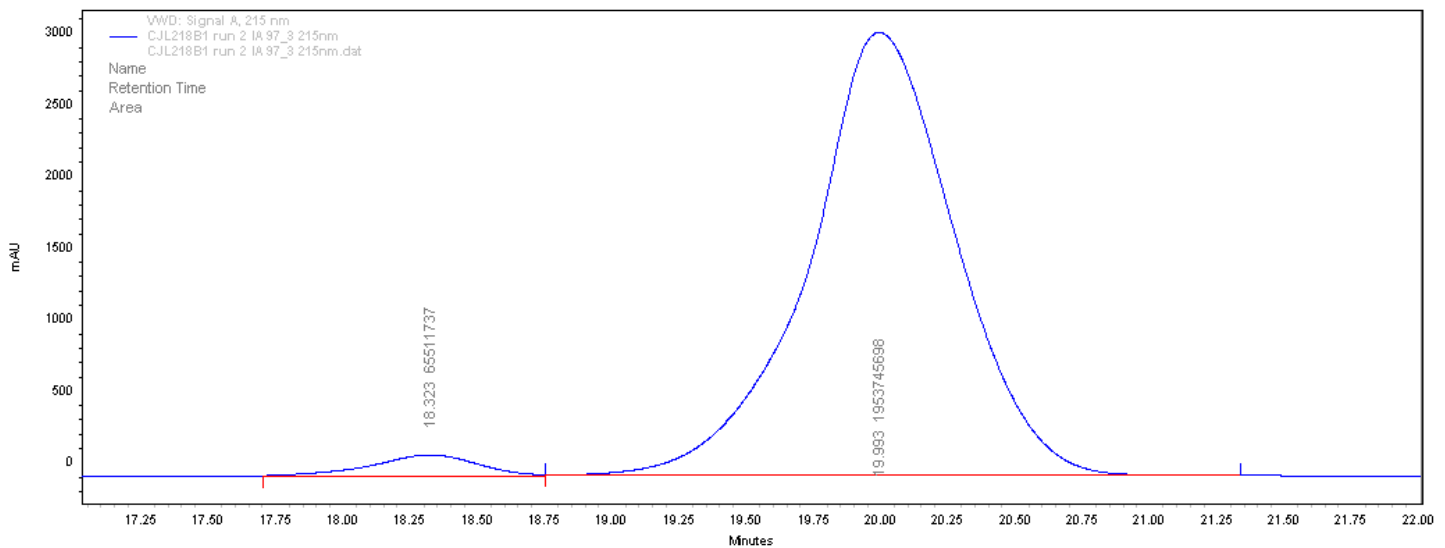

VWD : Signal A,

215 num Results

\begin{tabular}{rrrrr} 
Retention Time & Area & Axea \% & Height & Height \% \\
\hline 18.323 & 65511737 & 3.24 & 2429415 & 4.48 \\
19.993 & 1953745698 & 96.76 & 51836693 & 95.52
\end{tabular}

\begin{tabular}{|r|r|r|r|r|}
\hline Totals & 2019257435 & 100.00 & 54266108 & 100.00 \\
\hline
\end{tabular}


6-(4-Methoxyphenyl)-1,4,9-trimethyl-2,3-diphenyl-1,4,4a,6,7,8a-hexahydro-1,4methanonaphthalene-5,8-dione 5c

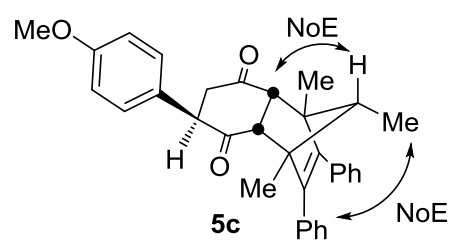

Major diastereomer confirmed by NOESY 2D NMR.

\section{Racemic procedure:}

General racemic procedure was followed with modifications and on a reduced scale, reacting p-methoxyphenylboronic acid 2a (18.4 mg, $0.12 \mathrm{mmol}, 2.4$ equiv.), Diels-Alder adduct $4 \mathbf{c}$ (18.4 mg, $0.050 \mathrm{mmol}, 1.0$ equiv., 7:1 d.r.), $\mathrm{Pd}(\mathrm{OAc})_{2}(1.1,0.010 \mathrm{mmol}, 0.10$ equiv.) and 1,10phenanthroline ( $1.1 \mathrm{mg}, 0.012 \mathrm{mmol}, 0.12$ equiv.) in $\mathrm{DMF}(0.5 \mathrm{~mL})$ under at an atmosphere of $\mathrm{O}_{2}$ at $40{ }^{\circ} \mathrm{C}$ for $24 \mathrm{~h}$. The resulting crude was purified by silica gel column chromatography (hexane:EtOAc 10:1 $\rightarrow$ 8:1) to yield $\mathbf{5 c}(13.6 \mathrm{mg}, 0.057 \mathrm{mmol}, 57 \%,>20: 1 \mathrm{dr}$ ) as an off-white powder.

\section{Enantioselective procedure:}

General enantioselective procedure B was followed with modifications on a reduced scale. Reacting $p$-methoxyphenylboronic acid 2a (18.4 mg, $0.12 \mathrm{mmol}, 2.4$ equiv.), Diels-Alder adduct $4 c$ (18.4 mg, $0.05 \mathrm{mmol}, 1.0$ equiv., 7:1 d.r.), $\mathrm{Pd}(\mathrm{OAc})_{2}(1.1 \mathrm{mg}, 0.010 \mathrm{mmol}, 0.05$ equiv.) and ${ }^{t} \mathrm{BuPyOx} \mathbf{L}-\mathbf{A}(1.2 \mathrm{mg}, 0.012 \mathrm{mmol}, 0.12$ equiv. $)$ in $\mathrm{DMF}(0.5 \mathrm{~mL})$ at $40{ }^{\circ} \mathrm{C}$ for 72 h. The resulting crude was purified by silica gel column chromatography (hexane:EtOAc 20:1 $\rightarrow$ 5:1) to yield $\mathbf{5 c}(17.3 \mathrm{mg}, 0.073 \mathrm{mmol}, 73 \%,>20: 1$ d.r., $93: 7$ e.r.) as a white powder solid. Mp: $75-77^{\circ} \mathrm{C}$ (hexane/chloroform); $\mathrm{R}_{\mathrm{f}}: 0.2$ in $10: 1$ pet. ether:EtOAc; $v \max / \mathrm{cm}^{-1}: 2960,2924$, 1700, 1612, 1516, 1489 1249, 1121, 742, 697; ${ }^{1} \mathrm{H}$ NMR (300 MHz, $\left.\mathrm{CDCl}_{3}\right) \delta 7.25-7.11(\mathrm{~m}$, $6 \mathrm{H}, \mathrm{Ar}-\mathrm{H}), 7.01$ (d, $J=8.5 \mathrm{~Hz}, 2 \mathrm{H}, \mathrm{Ar}-\mathrm{H}), 6.95-6.82(\mathrm{~m}, 6 \mathrm{H}, \mathrm{Ar}-\mathrm{H}), 3.90$ (dd, $J=10.3,5.1$ $\mathrm{Hz}, 1 \mathrm{H}, \mathrm{C} \underline{\mathrm{HAr}}$ ), 3.80 (s, 3H, $\left.\mathrm{OCH}_{3}\right), 3.21$ (d, $\left.J=9.8 \mathrm{~Hz}, 1 \mathrm{H}, \mathrm{CH}\right), 3.13$ (d, $J=9.8 \mathrm{~Hz}, 1 \mathrm{H}$, $\mathrm{CH}), 3.11(\mathrm{dd}, J=16.0,10.3 \mathrm{~Hz}, 1 \mathrm{H}, \underline{\mathrm{CHH}}) .2 .81(\mathrm{dd}, J=16.0,5.1 \mathrm{~Hz}, 1 \mathrm{H}, \mathrm{CH} \underline{\mathrm{H}}), 1.71(\mathrm{q}, J$ $\left.=6.3 \mathrm{~Hz}, 1 \mathrm{H}, \mathrm{CHCH}_{3}\right), 1.48\left(\mathrm{~s}, 3 \mathrm{H}, \mathrm{CH}_{3}\right), 1.40\left(\mathrm{~s}, 3 \mathrm{H}, \mathrm{CH}_{3}\right), 0.93\left(\mathrm{~d}, J=6.3 \mathrm{~Hz}, 3 \mathrm{H}, \mathrm{CHCH}_{3}\right)$. ; ${ }^{13} \mathrm{C}$ NMR (75 MHz, $\mathrm{CDCl}_{3}$ ) $\delta 208.8$ (C), 208.7 (C), 159.1 (C), 144.0 (C), 143.5 (C), 136.1 (C), $135.9(\mathrm{C}), 129.2(\mathrm{CH}), 129.0(\mathrm{CH}), 128.3(\mathrm{CH}), 128.19(\mathrm{C}), 128.15(\mathrm{CH}), 127.0(\mathrm{CH})$, $114.4(\mathrm{CH}), 66.6(\mathrm{CH}), 61.1(\mathrm{C}), 60.2(\mathrm{CH}), 59.0(\mathrm{CH}), 55.4\left(\mathrm{CH}_{3}\right), 52.3(\mathrm{CH}), 45.5\left(\mathrm{CH}_{2}\right)$, $16.7\left(\mathrm{CH}_{3}\right), 16.4\left(\mathrm{CH}_{3}\right), 7.7\left(\mathrm{CH}_{3}\right), 1 \times$ overlapping $\mathrm{C}$ signal, $2 \times$ overlapping $\mathrm{CH}$ signals; HRMS (TOF MS ASAP +) m/z calc. for $\mathrm{C}_{33} \mathrm{H}_{31} \mathrm{O} 3$ : $475.2273[\mathrm{M}-\mathrm{H}]^{+}$; found: 475.2269; 
$[\alpha]_{\mathrm{D}}^{20.5}=+20.0\left(\mathrm{c}\right.$ 0.5, $\left.\mathrm{CHCl}_{3}\right) ; 97: 3 \mathrm{er}$; HPLC (CHIRALPAK IA, hexane/2-propanol: 95:5,

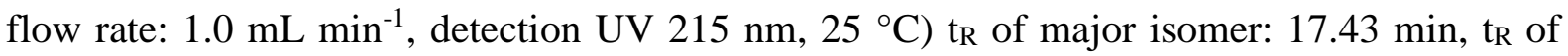
minor isomer: $15.71 \mathrm{~min}$.

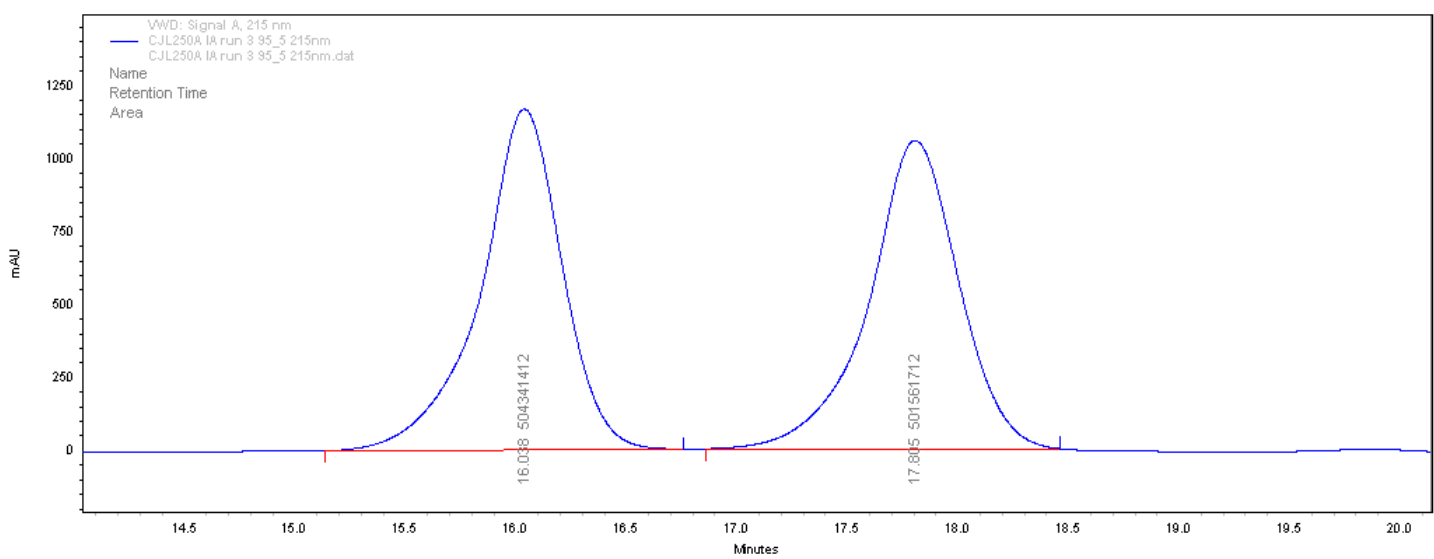

VwD: Signal A,

215 um Results

\begin{tabular}{rrrrr} 
Retention Time & Area & Area \% & Height & Height \% \\
\hline 16.038 & 504341412 & 50.14 & 19579235 & 52.50 \\
17.805 & 501561712 & 49.86 & 17714000 & 47.50
\end{tabular}

\begin{tabular}{|r|r|r|r|r|}
\hline Totals & 1005903124 & 100.00 & 37293235 & 100.00 \\
\hline
\end{tabular}

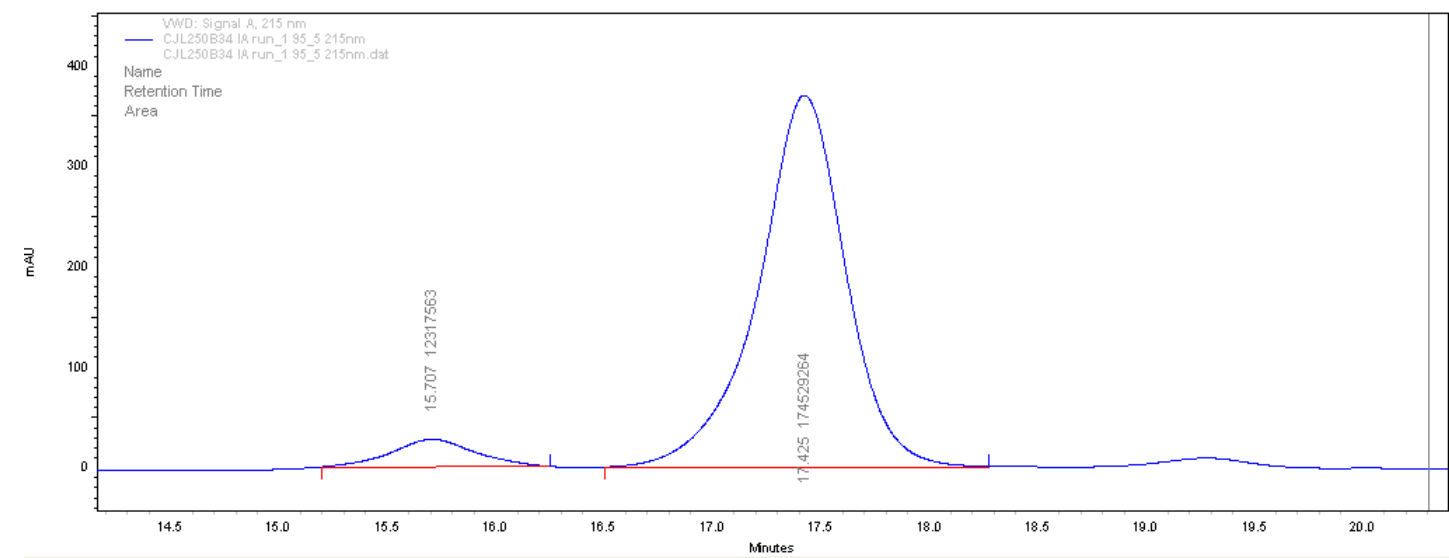

VWD : Signal A, 215 num Results Retention Time

\begin{tabular}{rrrrr} 
& Area & Area\% & Height & Height \% \\
\hline 15.707 & 12317563 & 6.59 & 458663 & 6.87 \\
17.425 & 174529264 & 93.41 & 6212992 & 93.13
\end{tabular}




\section{6-(4-Methoxyphenyl)-1,4-dimethyl-2,3-diphenyl-1,4,4a,6,7,8a-hexahydro-1,4- methanonaphthalene-5,8,9-trione $5 \mathrm{~d}$}

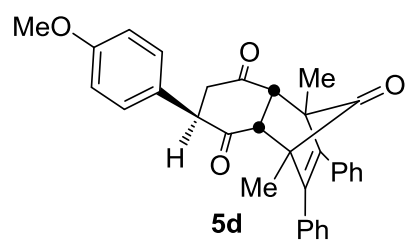

\section{Racemic procedure:}

General racemic procedure was followed. Reacting $p$-methoxyphenylboronic acid $\mathbf{2 a}(36.5 \mathrm{mg}$, 0.24 mmol, 2.4 equiv.), Diels-Alder adduct 4d (38.6 mg, 0.099 mmol, 1.0 equiv.), $\mathrm{Pd}(\mathrm{OAc})_{2}$ (1.1 mg, $0.005 \mathrm{mmol}, 0.05$ equiv.) and 1,10-phenanthroline (1.1 mg, $0.006 \mathrm{mmol}, 0.06$ equiv.) in DMF (1.0 mL) under at atmosphere of $\mathrm{O}_{2}$ (balloon) at $40{ }^{\circ} \mathrm{C}$ for $24 \mathrm{~h}$. The resulting crude was purified by silica gel column chromatography (pet. ether/EtOAc 20:1 $\rightarrow$ 5:1) to yield $\mathbf{5 d}$ (45.8 mg, $0.096 \mathrm{mmol}, 96 \%,>20: 1)$ as a white crystalline solid.

\section{Enantioselective procedure:}

General enantioselective procedure A was followed, reacting $p$-methoxyphenylboronic $2 \mathbf{a}$ (36.5 mg, 0.24 mmol, 2.4 equiv.), Diels-Alder adduct 4d (38.6 mg, 0.099 mmol, 1.0 equiv.), $\operatorname{Pd}(\mathrm{OAc}) 2$ (1.1 mg, $0.005 \mathrm{mmol}, 0.01$ equiv.) and ${ }^{t}$ BuPyOx L-A (1.2 mg, 0.006 mmol, 0.012 equiv.) in DMF (1.0 mL) under at an atmosphere of $\mathrm{O} 2$ at $40{ }^{\circ} \mathrm{C}$ for $72 \mathrm{~h}$. The resulting crude was purified by silica gel column chromatography (pet. ether/EtOAc $20: 1 \rightarrow 5: 1$ ) to yield $\mathbf{5 d}$ (32.3 mg, $0.068 \mathrm{mmol}, 68 \%$, >20:1 d.r., 94:6 e.r.) as a white crystalline solid.

Mp: $156-158^{\circ} \mathrm{C}$; Rf: 0.2 in 5:1 pet. ether:EtOAc; vmax $/ \mathrm{cm}^{-1}: 2961,2872,1776,1708,1613$, 1516, 1443, 1184, 780, 748, 698; ${ }^{1} \mathrm{H}$ NMR (300 MHz, $\left.\mathrm{CDCl}_{3}\right) 7.27$ - 7.16 (m, 6H, Ar-H), 7.05 - 6.99 (m, 2H, Ar-H), $6.95-6.87$ (m, 6H, Ar-H), 3.89 (dd, J = 9.9, 4.9 Hz, 1H, CㅌAr), 3.80 $\left(\mathrm{s}, 3 \mathrm{H}, \mathrm{OCH}_{3}\right), 3.30(\mathrm{~d}, J=10.6 \mathrm{~Hz}, 1 \mathrm{H}, \mathrm{CH}), 3.21(\mathrm{~d}, J=10.6 \mathrm{~Hz}, 1 \mathrm{H}, \mathrm{CH}), 3.20$ (dd, $J=$ 15.9, $9.9 \mathrm{~Hz}, 1 \mathrm{H}, \mathrm{C} \underline{\mathrm{HH}}), 2.85$ (dd, $J=15.9,4.9 \mathrm{~Hz}, 1 \mathrm{H}, \mathrm{CH} \underline{\mathrm{H}}), 1.58\left(\mathrm{~s}, 3 \mathrm{H}, \mathrm{CH}_{3}\right), 1.52$ (s, 3H, $\left.\mathrm{CH}_{3}\right) ; \delta{ }^{13} \mathrm{C}$ NMR (101 MHz, $\left.\mathrm{CDCl}_{3}\right) 206.0$ (C), 205.9 (C), 200.5 (C), 159.5 (C), 143.2 (C), $143.0(\mathrm{C}), 134.0(\mathrm{C}), 133.9(\mathrm{C}), 129.6(\mathrm{CH}), 129.4(\mathrm{CH}), 129.0(\mathrm{CH}), 128.5(\mathrm{CH}), 128.4(\mathrm{CH})$, $127.9(\mathrm{CH}), 127.8(\mathrm{CH}), 127.2(\mathrm{C}), 114.7(\mathrm{CH}), 58.1(\mathrm{C}), 58.0(\mathrm{C}), 55.5\left(\mathrm{CH}_{3}\right), 55.1(\mathrm{CH})$, $53.8(\mathrm{CH}), 52.6(\mathrm{CH}), 44.7\left(\mathrm{CH}_{2}\right), 12.4\left(\mathrm{CH}_{3}\right), 12.2\left(\mathrm{CH}_{3}\right)$; HRMS (TOF MS ASAP +) $\mathrm{m} / z$ calc. for $\mathrm{C}_{32} \mathrm{H}_{29} \mathrm{O}_{4}$ : $477.2066[\mathrm{M}+\mathrm{H}]^{+}$; found: 477.2063; $[\alpha]_{\mathrm{D}}^{23.2}=+46.6\left(\mathrm{c} 0.56, \mathrm{CHCl}_{3}\right) ; 94: 6$ e.r.; HPLC (CHIRALPAK IA, hexane/2-propanol: 95:5, flow rate: $1.0 \mathrm{~mL} \mathrm{~min}^{-1}$, detection UV $\left.215 \mathrm{~nm}, 25^{\circ} \mathrm{C}\right) \mathrm{t}_{\mathrm{R}}$ of major isomer: $18.24 \mathrm{~min}, \mathrm{t}_{\mathrm{R}}$ of minor isomer: $23.94 \mathrm{~min}$. 


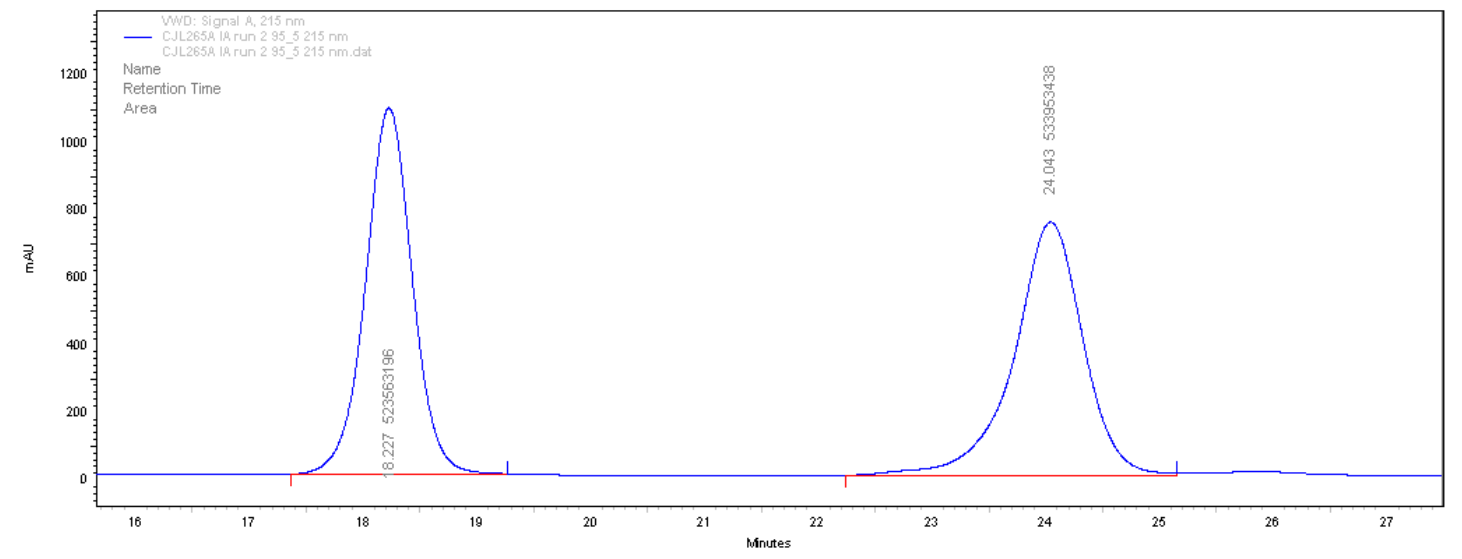

VWD : Signal A,

$215 \mathrm{um}$ Results

\begin{tabular}{rrrrr} 
Retention Time & Axea & Area \% & Height & Height \% \\
\hline 18.227 & 523563196 & 49.51 & 18259536 & 59.15 \\
24.043 & 533953438 & 50.49 & 12610053 & 40.85
\end{tabular}

\begin{tabular}{|r|r|r|r|r|}
\hline Totals & 1057516634 & 100.00 & 30869589 & 100.00 \\
\hline
\end{tabular}

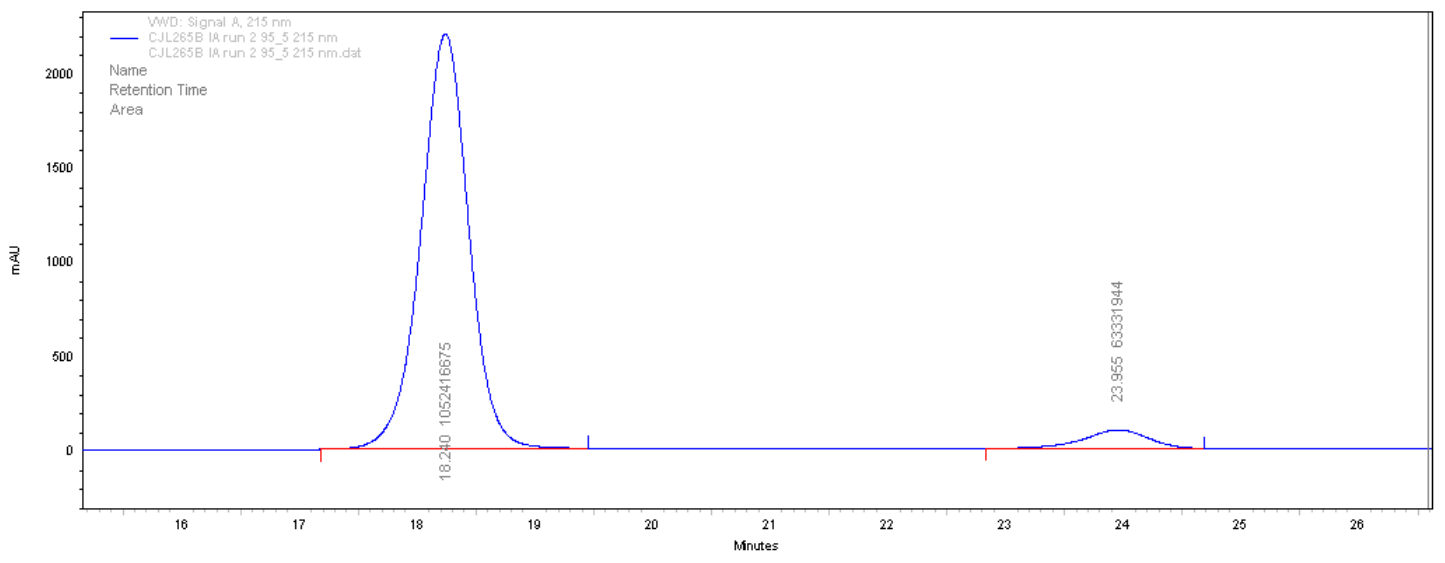

VWD : Signal A,

$215 \mathrm{~nm}$ Results

Retention Time

\begin{tabular}{rrrrr} 
Area & Area\% & Height & Height \% \\
\hline 18.240 & 1052416675 & 94.32 & 36934338 & 95.71
\end{tabular}

23.955

$\begin{array}{lll}63331944 & 5.68 & 1657386\end{array}$

95.71

\begin{tabular}{|r|r|r|r|r|}
\hline Totals & 1115748619 & 100.00 & 38591724 & 100.00 \\
\hline
\end{tabular}




\section{1,4-Diethyl-6-(4-methoxyphenyl)-2,3-diphenyl-1,4,4a,6,7,8a-hexahydro-1,4- methanonaphthalene-5,8,9-trione $5 \mathrm{e}$}

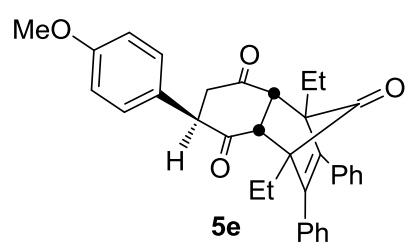

\section{Racemic procedure:}

General racemic procedure was followed, reacting $p$-methoxyphenylboronic acid 2a (36.5 mg, $0.24 \mathrm{mmol}, 2.4$ equiv.), Diels-Alder adduct $4 \mathrm{e}$ (39.6 mg, $0.099 \mathrm{mmol}, 1.0$ equiv.), $\mathrm{Pd}(\mathrm{OAc})_{2}$ (1.1 mg, $0.005 \mathrm{mmol}, 0.01$ equiv.) and 1,10-phenanthroline (1.1 mg, $0.006 \mathrm{mmol}, 0.012$ equiv.) in DMF (1.0 mL) under at an atmosphere of $\mathrm{O}_{2}$ at $40{ }^{\circ} \mathrm{C}$ for $24 \mathrm{~h}$. The resulting crude was purified by silica gel column chromatography (pet. ether/EtOAc 5:1) to yield 5e (49.2 mg, $0.097 \mathrm{mmol}, 97 \%,>20: 1$ d.r.) as a white crystalline solid.

\section{Enantioselective procedure:}

General enantioselective procedure A was followed. Reacting $p$-methoxyphenylboronic acid 2a (36.5 mg, 0.24 mmol, 2.4 equiv.), Diels-Alder adduct 4e (39.6 mg, 0.099 mmol, 1.0 equiv.), $\mathrm{Pd}(\mathrm{OAc})_{2}\left(1.1 \mathrm{mg}, 0.005 \mathrm{mmol}, 0.01\right.$ equiv.) and ${ }^{t} \mathrm{BuPyOx} \mathbf{L}-\mathbf{A}$ (1.2 mg, $0.006 \mathrm{mmol}, 0.012$ equiv.) in DMF (1.0 mL) under at an atmosphere of $\mathrm{O}_{2}$ at $40{ }^{\circ} \mathrm{C}$ for $72 \mathrm{~h}$. The resulting crude was purified by silica gel column chromatography (hexanes/EtOAc 10:1 $\rightarrow$ 7.5:1) to yield 5e (36.4 mg, $0.074 \mathrm{mmol}, 72 \%,>20: 1$ d.r., 92:8 e.r.) as an off-white solid.

Mp: $145{ }^{\circ} \mathrm{C}$ (decomp.) (hexanes/chloroform); $\mathrm{R}_{\mathrm{f}}$ : 0.2 in 7.5:1 pet. ether:EtOAc; $v \mathrm{max} / \mathrm{cm}^{-1}$ : 2969, 1770, 1705, 1612,1513, 1487, 1252, 1180, 820, 732, 699; ${ }^{1} \mathrm{H}$ NMR (300 MHz, $\mathrm{CDCl}_{3}$ ) $7.23-7.14$ (m, 6H, Ar-H), $7.09-7.00$ (m, 2H, Ar-H), $6.98-6.87$ (m, 6H, Ar-H), 3.89 (dd, $J$ $=9.9,4.8 \mathrm{~Hz}, 1 \mathrm{H}, \mathrm{C} \underline{\mathrm{HAr}}), 3.81\left(\mathrm{~s}, 3 \mathrm{H}, \mathrm{OCH}_{3}\right), 3.57(\mathrm{~d}, J=10.6 \mathrm{~Hz}, 1 \mathrm{H}, \mathrm{CH}), 3.50(\mathrm{~d}, J=10.6$ Hz, 1H, CH), 3.23 (dd, $J=15.8,9.9 \mathrm{~Hz}, 1 \mathrm{H}, \mathrm{C} \underline{\mathrm{HH}}), 2.87$ (dd, $J=15.8,4.8 \mathrm{~Hz}, 1 \mathrm{H}), \mathrm{C} \underline{\mathrm{HH}}, 2.09$ $\left(\mathrm{dt}, J=14.4,7.4 \mathrm{~Hz}, 4 \mathrm{H}, \mathrm{CHHCH}_{3}\right), 0.97\left(\mathrm{t}, J=7.4 \mathrm{~Hz}, 3 \mathrm{H}, \mathrm{CH}_{3}\right), 0.89$ (t, $J=7.4 \mathrm{~Hz}, 3 \mathrm{H}$, $\left.\mathrm{CH}_{3}\right) ; \delta{ }^{13} \mathrm{C}$ NMR (75 MHz, $\left.\mathrm{CDCl}_{3}\right) 206.41(\mathrm{C}), 206.36$ (C), 199.8 (C), 159.3 (C), 143.7 (C), $143.3(\mathrm{C}), 134.1(\mathrm{C}), 134.0(\mathrm{C}), 129.3(\mathrm{CH}), 129.2(\mathrm{CH}), 128.8(\mathrm{CH}), 128.3(\mathrm{CH}), 128.2(\mathrm{CH})$, $127.7(\mathrm{CH}), 127.6(\mathrm{CH}), 127.1(\mathrm{C}), 114.6(\mathrm{C}), 61.34(\mathrm{C}), 61.29(\mathrm{C}), 55.3\left(\mathrm{CH}_{3}\right), 52.5(\mathrm{CH})$, $49.9(\mathrm{CH}), 49.1(\mathrm{CH}), 44.6\left(\mathrm{CH}_{2}\right), 19.2\left(\mathrm{CH}_{3}\right), 19.1\left(\mathrm{CH}_{3}\right), 9.4\left(\mathrm{CH}_{3}\right), 9.3\left(\mathrm{CH}_{3}\right) ; \mathrm{HRMS}(\mathrm{TOF}$ MS ASAP +) $m / z$ calc. for $\mathrm{C}_{34} \mathrm{H}_{31} \mathrm{O}_{4}: 503.2222[\mathrm{M}-\mathrm{H}]^{+}$; found: $503.2218 ;[\alpha]_{\mathrm{D}}^{22.6}=+46.5(\mathrm{c}$ 1.0, $\mathrm{CHCl}_{3}$ ); 92:8 e.r.; HPLC (CHIRALPAK IB, hexane/2-propanol: 95:5, flow rate: $1.0 \mathrm{~mL}$ 
$\min ^{-1}$, detection UV $215 \mathrm{~nm}, 25^{\circ} \mathrm{C}$ ) $\mathrm{t}_{\mathrm{R}}$ of major isomer: $14.8 \mathrm{~min}, \mathrm{t}_{\mathrm{R}}$ of minor isomer: 18.1 $\min$.

\section{Enantioselective scale-up (1 mmol) procedure:}

In a dry round bottom flask $(25 \mathrm{~mL}), \mathrm{Pd}(\mathrm{OAc})_{2}(11.2 \mathrm{mg}, 0.050 \mathrm{mmol}, 0.05$ equiv. $)$ and ${ }^{t}$ BuPyOx L-A (12.3 mg, $0.060 \mathrm{mmol}, 0.06$ equiv.) were pre-mixed in DMF (3 mL) for 15 minutes. Diels-Alder adduct 4e (396.5 mg, $1.00 \mathrm{mmol}, 1.0$ equiv.) and p-methoxyphenyl boronic acid 2a (364.8 mg, $2.40 \mathrm{mmol}, 2.40$ equiv.) were added, along with DMF (7 mL). The reaction mixture was stirred at $40{ }^{\circ} \mathrm{C}$ under an atmosphere of oxygen (balloon) for $72 \mathrm{~h}$. The reaction was then diluted with 2:1 $\mathrm{Et}_{2} \mathrm{O}: \mathrm{EtOAc}(45 \mathrm{~mL})$ and the organic layer was washed with $\mathrm{H}_{2} \mathrm{O}(5 \times 5 \mathrm{~mL})$ and brine $(1 \times 10 \mathrm{~mL})$, the combined organic layers were dried over $\mathrm{MgSO}_{4}$ and solvent was removed under reduced pressure. The resulting crude was purified by silica gel column chromatography (gradient 20:1 hexanes:EtOAc $+10 \%$ toluene $\rightarrow$ 5:1 hexanes:EtOAc $+10 \%$ toluene) to yield $\mathbf{5 e}(304.6 \mathrm{mg}, 603.6 \mathrm{mmol}, 60 \%,>20: 1$ d.r., $93: 7$ e.r. $)$ as a pale yellow fluffy solid.

For characterisation, please see the enatioselective procedure above.

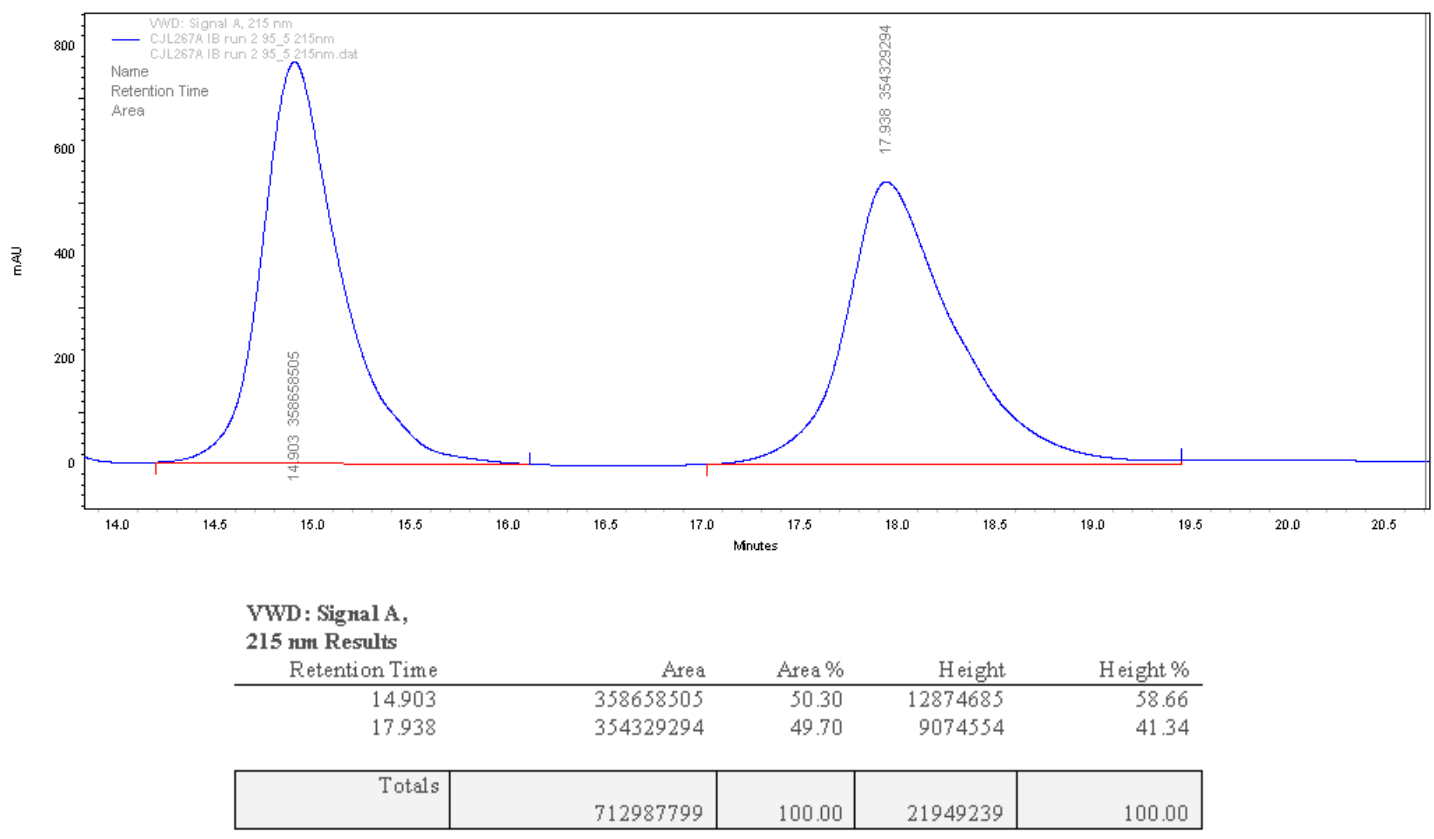


HPLC trace for $0.1 \mathrm{mmol}$ scale:

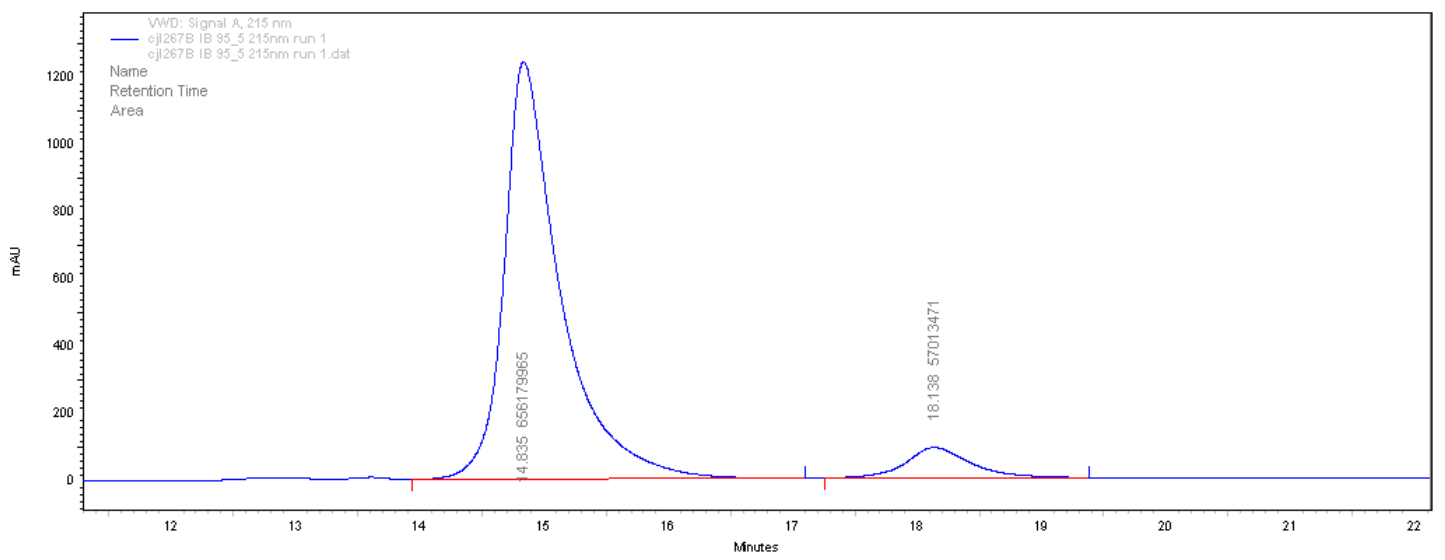

VWD : Signal A

215 num Results

\begin{tabular}{rrrrr} 
Retention Time & Area & Area\% & Height & Height \% \\
\hline 14.835 & 656179965 & 92.01 & 20826138 & 93.17 \\
18.138 & 57013471 & 7.99 & 1527778 & 6.83
\end{tabular}

\begin{tabular}{|r|r|r|r|r|}
\hline Totals & 713193436 & 100.00 & 22353916 & 100.00 \\
\hline
\end{tabular}

HPLC trace for $1.0 \mathrm{mmol}$ scale:

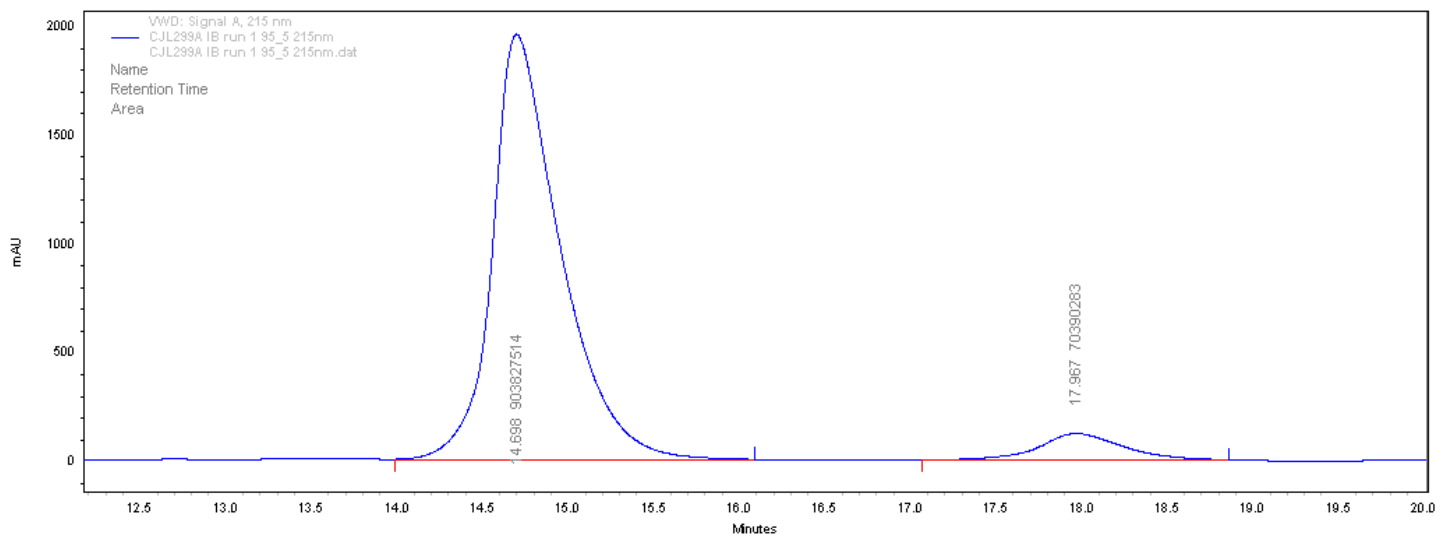

VWD: Signal A,

215 um Results

\begin{tabular}{rrrrrr} 
Retention Time & Area & Area \% & Height & Height \% \\
\hline 14.698 & 903827514 & 92.77 & 32776604 & 94.13 \\
17.967 & 70390283 & 7.23 & 2045609 & 5.87 \\
\hline \multicolumn{2}{l}{ Totals } & 974217797 & 100.00 & 34822213 & 100.00 \\
\hline
\end{tabular}




\section{6-(4-Methoxyphenyl)-1,2,3,4-tetramethyl-1,4,4a,6,7,8a-hexahydro-1,4- methanonaphthalene-5,8-dione $5 f$}

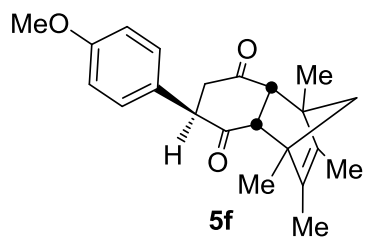

\section{Racemic procedure:}

General racemic procedure was followed. Reacting $p$-methoxyphenylboronic acid $\mathbf{2 a}(36.5 \mathrm{mg}$, $0.24 \mathrm{mmol}, 2.4$ equiv.), Diels-Alder adduct $4 \mathbf{f}$ (23.0 mg, $0.099 \mathrm{mmol}, 1.0$ equiv.), $\mathrm{Pd}(\mathrm{OAc})_{2}$ ( $1.1 \mathrm{mg}, 0.005 \mathrm{mmol}, 0.05$ equiv.) and 1,10-phenanthroline ( $1.1 \mathrm{mg}, 0.006 \mathrm{mmol}, 0.06$ equiv.) in DMF (1.0 mL) under at atmosphere of $\mathrm{O}_{2}$ (balloon) at $40{ }^{\circ} \mathrm{C}$ for $24 \mathrm{~h}$. The resulting crude was purified by silica gel column chromatography (hexane:EtOAc 20:1 $\rightarrow$ 10:1) to yield $\mathbf{5 f}$ (25.1 mg, $0.077 \mathrm{mmol}, 77 \%,>20: 1$ d.r.) as a white powder solid.

\section{Enantioselective procedure:}

General enantioselective procedure $\mathrm{C}$ was followed with modifications. Reacting recrystallised p-methoxyphenylboronic acid 2a (30.6 mg, $0.20 \mathrm{mmol}, 2.0$ equiv.), Diels-Alder adduct $\mathbf{4 f}$ (35.4 mg, 0.099 mmol, 1.0 equiv.), $\mathrm{Pd}(\mathrm{OTFA})_{2}(1.7 \mathrm{mg}, 0.005 \mathrm{mmol}, 0.05$ equiv.) and 5$\mathrm{CF}_{3}{ }^{t}$ BuPyOx L-B (1.6 mg, $0.006 \mathrm{mmol}, 0.06$ equiv.) in DCE $(0.5 \mathrm{~mL})$ at $40{ }^{\circ} \mathrm{C}$ for $72 \mathrm{~h}$. The resulting crude was purified by silica gel column chromatography (hexane:EtOAc 15:1 $\rightarrow$ 10:1) to yield $\mathbf{5 f}(22.3 \mathrm{mg}, 0.068 \mathrm{mmol}, 68 \%,>20: 1$ d.r., $95: 5$ e.r.) as a white powder.

Mp: $123-125^{\circ} \mathrm{C}$ (hexane/chloroform); ${ }^{\circ} \mathrm{C}$; $\mathrm{R}_{\mathrm{f}}$ : 0.2 in 10:1 pet. ether:EtOAc; vmax/ $\mathrm{cm}^{-1}: 2926$, 2859, 1702, 1609, 1512, 1445, 1249, 1181, 839; ${ }^{1} \mathrm{H}$ NMR (300 MHz, $\left.\mathrm{CDCl}_{3}\right) \delta 7.03-6.97(\mathrm{~m}$, $2 \mathrm{H}, \mathrm{Ar}-\mathrm{H}), 6.88-6.82(\mathrm{~m}, 2 \mathrm{H}, \mathrm{Ar}-\mathrm{H}), 3.78\left(\mathrm{~s}, 3 \mathrm{H}, \mathrm{OCH}_{3}\right), 3.45(\mathrm{dd}, J=9.7,4.9 \mathrm{~Hz}, 1 \mathrm{H}$, CㅂA), 2.97 (d, $J=9.4 \mathrm{~Hz}, 1 \mathrm{H}, \mathrm{CH}), 2.93(\mathrm{dd}, J=15.9,9.7 \mathrm{~Hz}, 1 \mathrm{H}, \mathrm{C} \underline{H} \mathrm{H}), 2.88$ (d, $J=9.4$ $\mathrm{Hz}, 1 \mathrm{H}, \mathrm{CH}), 2.49$ (dd, J=15.9, 4.9 Hz, 1H, $\mathrm{CH} \underline{\mathrm{H}}), 1.62$ (s, 3H, $\left.\mathrm{CH}_{3}\right), 1.61$ (s, 3H, CH 3 ), 1.48

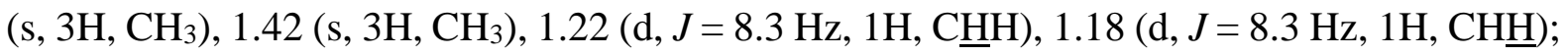
${ }^{13} \mathrm{C}$ NMR (101 MHz, $\left.\mathrm{CDCl}_{3}\right) \delta 209.3$ (C), 208.9 (C), 159.0 (C), 138.9 (C), 138.6 (C), 129.0 $(\mathrm{CH}), 128.1(\mathrm{C}), 114.4(\mathrm{CH}), 62.7\left(\mathrm{CH}_{2}\right), 59.3(\mathrm{CH}), 58.3(\mathrm{CH}), 57.1(\mathrm{C}), 57.0(\mathrm{C}), 55.4\left(\mathrm{CH}_{3}\right)$, $52.0(\mathrm{CH}), 44.8\left(\mathrm{CH}_{2}\right), 17.2\left(\mathrm{CH}_{3}\right), 17.0\left(\mathrm{CH}_{3}\right), 11.7\left(\mathrm{CH}_{3}\right), 11.5\left(\mathrm{CH}_{3}\right)$; HRMS (TOF MS ASAP +) $m / z$ calc. for $\mathrm{C}_{22} \mathrm{H}_{25} \mathrm{O}_{3}: 337.1804[\mathrm{M}+\mathrm{H}]^{+}$; found: $337.1805 ;[\alpha]_{\mathrm{D}}^{22.0}=+54.8(\mathrm{c} 1.0$, $\mathrm{CHCl}_{3}$ ); 95:5 e.r.; HPLC (CHIRALPAK IB, hexane/2-propanol: 97:3, flow rate: $1.0 \mathrm{~mL} \mathrm{~min}{ }^{-}$ ${ }^{1}$, detection UV $215 \mathrm{~nm}, 25^{\circ} \mathrm{C}$ ) $\mathrm{t}_{\mathrm{R}}$ of major isomer: $9.1 \mathrm{~min}, \mathrm{t}_{\mathrm{R}}$ of minor isomer: $11.7 \mathrm{~min}$. 


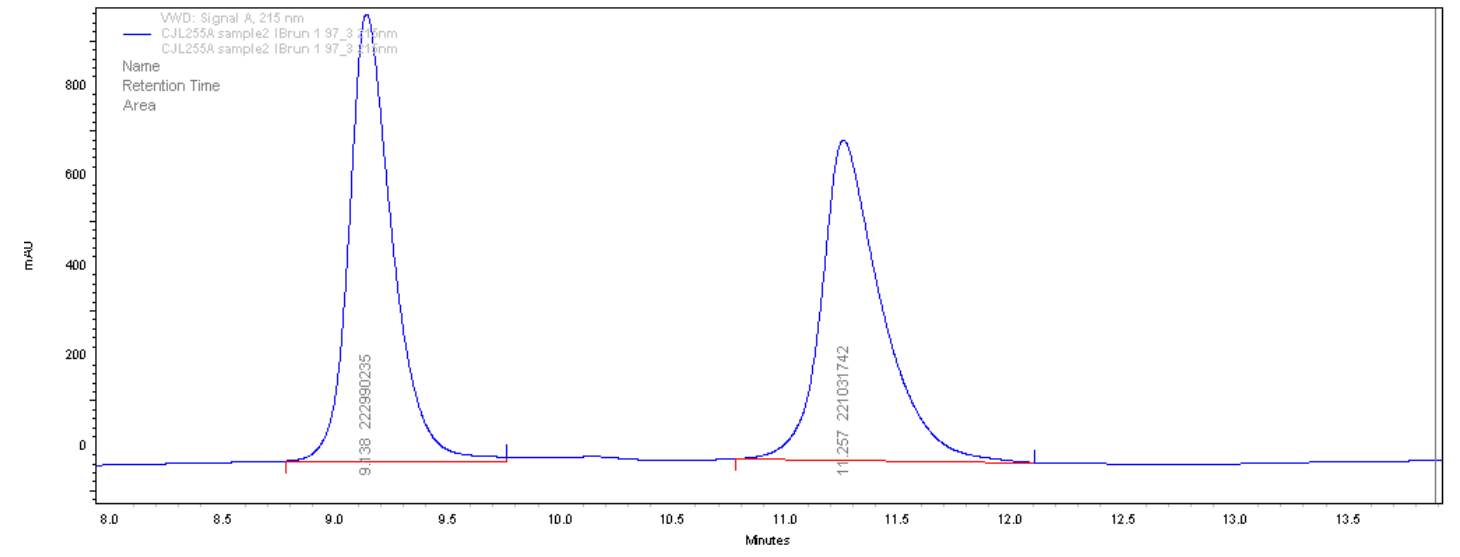

VWD: Signal A,

215 num Results

\begin{tabular}{rrrrr}
$215 \mathrm{~mm}$ Results & Area & Area \% & Height & Height \% \\
\hline Retention Time & 222990235 & 50.22 & 16660880 & 58.26 \\
11.257 & 221031742 & 49.78 & 11936449 & 41.74
\end{tabular}

\begin{tabular}{|r|r|r|r|r|}
\hline Totals & 444021977 & 100.00 & 28597329 & 100.00 \\
\hline
\end{tabular}

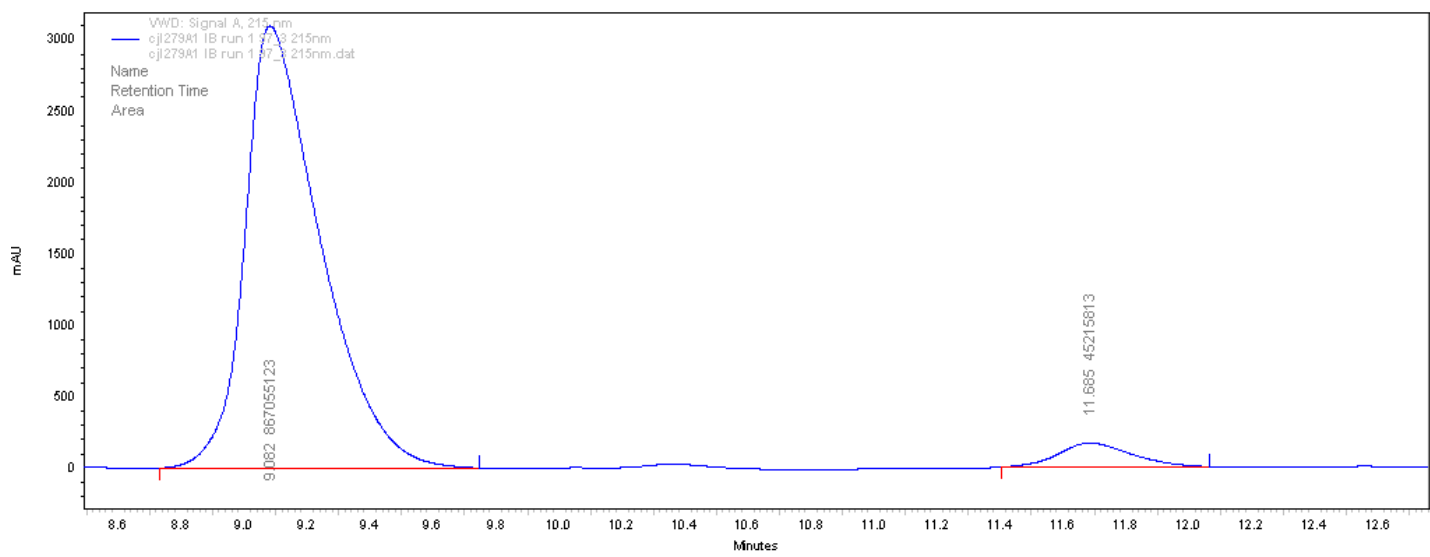

VWD: Signal A,

215 Jum Results

Retention Time

\begin{tabular}{rrrrr}
9.082 & Area & Area $\%$ & Height & He1ght \% \\
\hline 1.685 & 867055123 & 95.04 & 51954124 & 94.88
\end{tabular}

11.685

45215813

$496 \quad 2802255$

5.12

\begin{tabular}{|r|r|r|r|r|}
\hline Totals & 912270936 & 100.00 & 54756379 & 100.00 \\
\hline
\end{tabular}




\section{6-(4-Methoxyphenyl)-1,2,3,4,9-pentamethyl-1,4,4a,6,7,8a-hexahydro-1,4- methanonaphthalene-5,8-dione 5g}

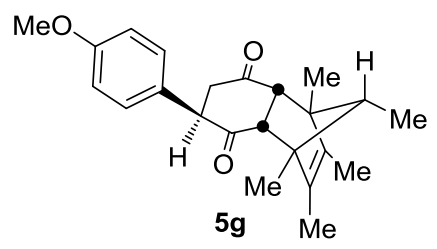

\section{Racemic procedure:}

General racemic procedure was followed with modifications and on a reduced scale, reacting p-methoxyphenylboronic acid 2a (18.2 mg, $0.12 \mathrm{mmol}, 2.4$ equiv.), Diels-Alder adduct $\mathbf{4 g}$ (12.2mg, 0.050 mmol, 1.0 equiv., 20:1 d.r.), $\mathrm{Pd}(\mathrm{OAc})_{2}$ (1.1, $0.005 \mathrm{mmol}, 0.01$ equiv.) and 1,10phenanthroline ( $1.1 \mathrm{mg}, 0.006 \mathrm{mmol}, 0.012$ equiv.) in DMF (1.0 mL) under at an atmosphere of $\mathrm{O}_{2}$ at $40{ }^{\circ} \mathrm{C}$ for $24 \mathrm{~h}$. The resulting crude was purified by silica gel column chromatography (hexane:EtOAc 20:1 $\rightarrow$ 10:1) to yield $\mathbf{5 g}(15.2 \mathrm{mg}, 0.086 \mathrm{mmol}, 86 \%,>20: 1$ d.r.) as a white crystalline powder.

\section{Enantioselective procedure:}

General enantioselective procedure $\mathrm{C}$ was followed. Reacting recrystallised $p$ methoxyphenylboronic acid 2a (30.6 mg, 0.20 mmol, 2.0 equiv.), Diels-Alder adduct $4 \mathbf{g}$ (24.4 mg, 0.099 mmol, 1.0 equiv.), $\mathrm{Pd}(\mathrm{OTFA})_{2}(1.7 \mathrm{mg}, 0.005 \mathrm{mmol}, 0.05$ equiv.) and 5$\mathrm{CF}_{3}{ }^{t} \mathrm{BuPyOx} \mathbf{L}-\mathrm{B}\left(1.6 \mathrm{mg}, 0.006 \mathrm{mmol}, 0.06\right.$ equiv.) in DCE $(0.5 \mathrm{~mL})$ at $40{ }^{\circ} \mathrm{C}$ for $72 \mathrm{~h}$. The resulting crude was purified by silica gel column chromatography (hexane:EtOAc 15:1 $\rightarrow$ 10:1) to yield $\mathbf{5 g}$ (18.9 $\mathrm{mg}, 0.054 \mathrm{mmol}, 64 \%,>20: 1$ d.r., $95: 5$ e.r.) as a white powder.

Mp: $126-129^{\circ} \mathrm{C}$ (hexane/chloroform); $\mathrm{R}_{\mathrm{f}}: 0.2$ in 10:1 pet. ether:EtOAc; vmax/ $\mathrm{cm}^{-1}$ : 2956, 2928, 2868, 1702, 1699, 1652, 1512, 1445, 1250, 1182, 841, ; ${ }^{1} \mathrm{H}$ NMR (300 MHz, $\left.\mathrm{CDCl}_{3}\right)$ $7.04-6.97$ (m, 2H, Ar-H), $6.91-6.82$ (m, 2H, Ar-H), 3.78 (s, 3H, OCH $), 3.45$ (dd, J = 9.9, $5.0 \mathrm{~Hz}, 1 \mathrm{H}, \mathrm{C} \underline{\mathrm{HAr}}), 2.91$ (dd, $J=15.8,9.9 \mathrm{~Hz}, 1 \mathrm{H}, \mathrm{C} \underline{\mathrm{H}}$ ), 2.89 (d, $J=9.2 \mathrm{~Hz}, 1 \mathrm{H}, \mathrm{CH}), 2.80$ (d, $J=9.2 \mathrm{~Hz}, 1 \mathrm{H}, \mathrm{CH}), 2.49(\mathrm{dd}, J=15.8,5.0 \mathrm{~Hz}, 1 \mathrm{H}, \mathrm{CH} \underline{\mathrm{H}}), 1.58\left(\mathrm{~s}, 3 \mathrm{H}, \mathrm{CH}_{3}\right), 1.57$ (s, 3H, $\left.\mathrm{CH}_{3}\right), 1.35$ (s, 3H, $\left.\mathrm{CH}_{3}\right), 1.33$ (d, $\left.J=6.4 \mathrm{~Hz}, 2 \mathrm{H}, \mathrm{CHCH}_{3}\right), 1.29$ (s, 3H, $\left.\mathrm{CH}_{3}\right), 0.56$ (d, $J=6.4$ $\mathrm{Hz}, 3 \mathrm{H}, \mathrm{CHCH} 3$ ); $\delta{ }^{13} \mathrm{C}$ NMR (101 MHz, $\mathrm{CDCl}_{3}$ ) 209.4 (C), 209.0 (C), 159.0 (C), 135.3 (C), $134.9(\mathrm{C}), 129.0(\mathrm{CH}), 128.3(\mathrm{C}), 114.4(\mathrm{CH}), 63.3(\mathrm{CH}), 60.1(\mathrm{C}), 59.9(\mathrm{C}), 59.4(\mathrm{CH}), 58.4$ $(\mathrm{CH}), 55.4\left(\mathrm{CH}_{3}\right), 52.2(\mathrm{CH}), 45.0\left(\mathrm{CH}_{2}\right), 14.8\left(\mathrm{CH}_{3}\right), 14.5\left(\mathrm{CH}_{3}\right), 12.1\left(\mathrm{CH}_{3}\right), 11.8\left(\mathrm{CH}_{3}\right), 7.4$ $\left(\mathrm{CH}_{3}\right) ; \delta$ HRMS (TOF MS ASAP +) m/z calc. for $\mathrm{C}_{23} \mathrm{H}_{27} \mathrm{O}_{3}: 351.1960[\mathrm{M}+\mathrm{H}]^{+}$; found: 351.1960; $[\alpha]_{\mathrm{D}}^{20.6}=+66.0\left(\mathrm{c} 1.18, \mathrm{CHCl}_{3}\right)$; 95:5 e.r.; HPLC (CHIRALPAK IA, hexane/2- 
propanol: $95: 5$, flow rate: $1.0 \mathrm{~mL} \min ^{-1}$, detection $\mathrm{UV} 215 \mathrm{~nm}, 25^{\circ} \mathrm{C}$ ) $\mathrm{t}_{\mathrm{R}}$ of major isomer: 10.6 $\min , t_{R}$ of minor isomer: $10.0 \mathrm{~min}$.

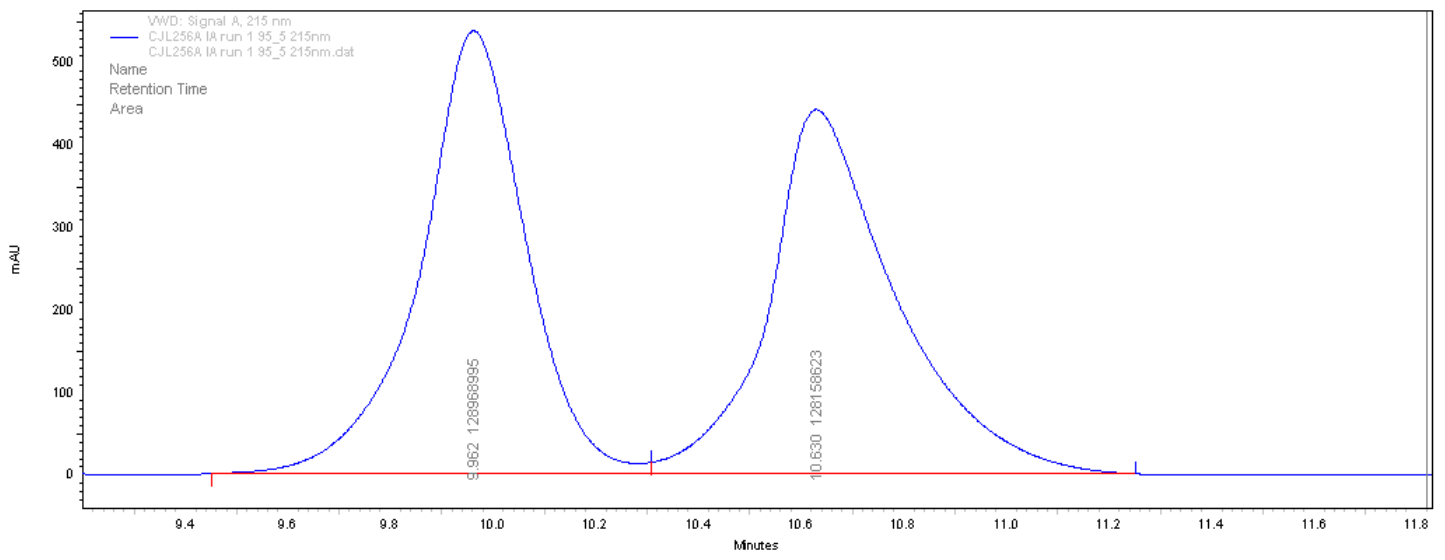

VWD : Signal A,

$215 \mathrm{~nm}$ Results

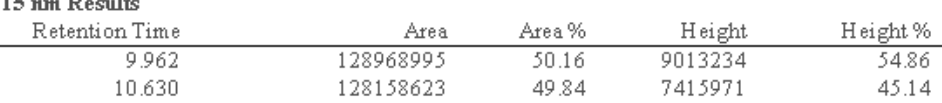

10630

$128158623 \quad 4984 \quad 741597$

45.14

\begin{tabular}{|r|r|r|r|r|}
\hline Totals & 257127618 & 100.00 & 16429205 & 100.00 \\
\hline
\end{tabular}

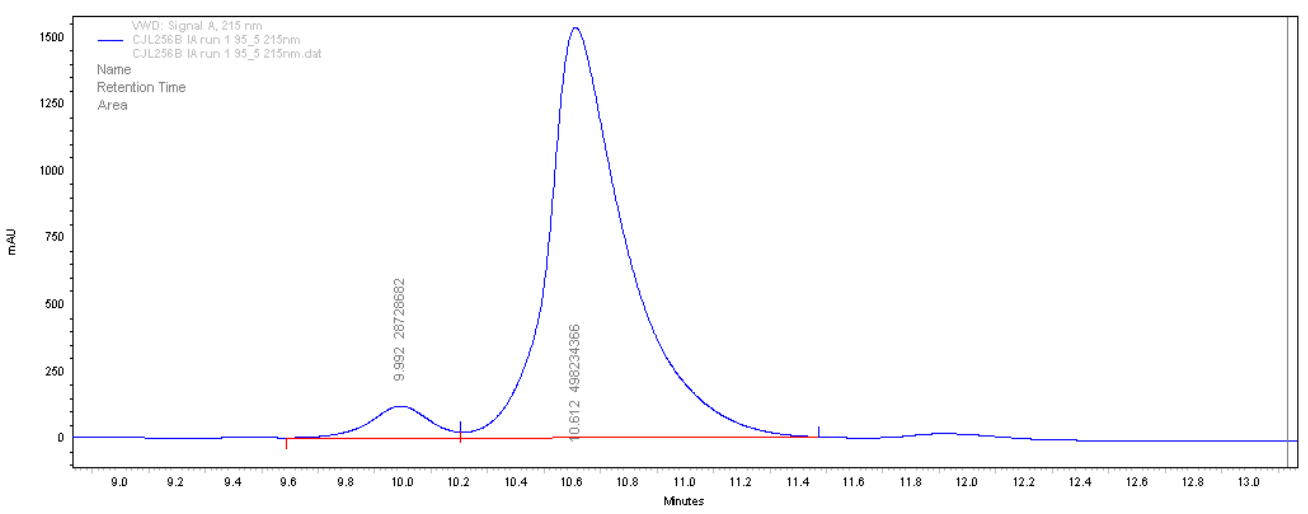

VWD : Signal A,

$215 \mathrm{um}$ Results

Retention Time

\begin{tabular}{rrrrr} 
Area & Area\% & Height & Height \% \\
\hline 9.992 & 28728682 & 5.45 & 1982855 & 7.16 \\
10.612 & 498234366 & 94.55 & 25728465 & 92.84
\end{tabular}

Totals 


\section{4-(4-Methoxyphenyl)-9,10-dihydro-9,10-[1,2]benzenoanthracene-13,16-dione 5 h}
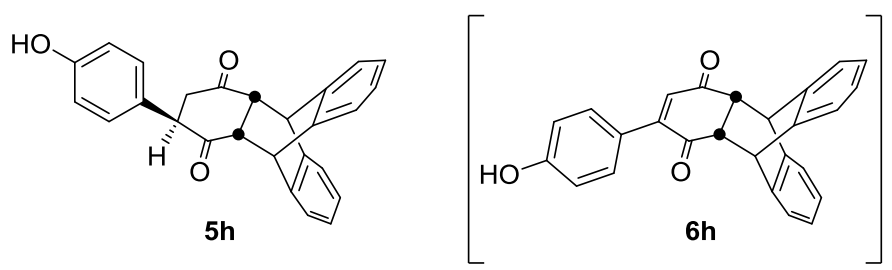

p-Hydroxyphenylboronic acid $\mathbf{2} \mathbf{m}$ was used instead of $\mathbf{2 a}$, as CSP-HPLC separating conditions could not be found for the product when $p$-methoxyphenylboronic acid $\mathbf{2 a}$ was used.

\section{Racemic procedure:}

General racemic procedure was followed. Reacting $p$-hydroxyphenylboronic acid $2 \mathbf{m}$ (33.1 mg, 0.24 mmol, 2.4 equiv.), Diels-Alder adduct 4h (28.6 mg, $0.099 \mathrm{mmol}, 1.0$ equiv.), $\mathrm{Pd}(\mathrm{OAc})_{2}(1.1 \mathrm{mg}, 0.005 \mathrm{mmol}, 0.01$ equiv.) and 1,10-phenanthroline (1.1 mg, $0.006 \mathrm{mmol}$, 0.012 equiv.) in DMF (1.0 mL) under at an atmosphere of $\mathrm{O}_{2}$ at $40{ }^{\circ} \mathrm{C}$ for $24 \mathrm{~h} .$. The resulting crude was purified by silica gel column chromatography (pet. ether/EtOAc 3:1) to yield a 2:1 mixture of $\mathbf{5 h : 6 h}(37.5 \mathrm{mg}, 0.098 \mathrm{mmol}, 98 \%)$ as a yellow oil.

\section{Enantioselective procedure:}

General enantioselective procedure $\mathrm{C}$ was followed with modifications. Reacting $p$ hydroxyphenylboronic acid 2m (33.1mg, 0.24 mmol, 2.4 equiv.), Diels-Alder adduct $4 \mathbf{h}$ (28.6 mg, 0.099 mmol, 1.0 equiv.), Pd(OTFA) $)_{2}(1.7 \mathrm{mg}, 0.005 \mathrm{mmol}, 0.05$ equiv.) and 4$\mathrm{CF}_{3}{ }^{t} \mathrm{BuPyOx} \mathbf{L}-\mathbf{C}\left(1.2 \mathrm{mg}, 0.006 \mathrm{mmol}, 0.006 \mathrm{mmol}, 0.06\right.$ equiv.) in DCE (0.5 mL) at $40{ }^{\circ} \mathrm{C}$ for $48 \mathrm{~h}$. The resulting crude was purified by silica gel column chromatography (hexanes/EtOAc 5:1 $\rightarrow$ 3:1) to yield $\mathbf{5 h}(24.7 \mathrm{mg}, 0.065 \mathrm{mmol}, 65 \%,>20: 1$ d.r., 85:15 e.r.) as an off-white powder.

Mp: $107-109{ }^{\circ} \mathrm{C}$ (decomp.) (hexanes/chloroform); $\mathrm{R}_{\mathrm{f}}$ : 0.2 in 3:1 pet. ether:EtOAc; $v \mathrm{max} / \mathrm{cm}^{-}$ ${ }^{1}: 3465,3013,1698,1614,1595,1516,1204,1148,842,774,750 ;{ }^{1} \mathrm{H} \mathrm{NMR}\left(300 \mathrm{MHz}, \mathrm{CDCl}_{3}\right)$ $\delta 7.35(\mathrm{dd}, J=5.4,3.2 \mathrm{~Hz}, 2 \mathrm{H}, \mathrm{Ar}-\mathrm{H}), 7.33-7.28$ (m, 2H, Ar-H), $7.19-7.15$ (m, 2H, Ar-H), $7.14(\mathrm{dd}, J=5.4,3.2 \mathrm{~Hz}, 2 \mathrm{H}, \mathrm{Ar}-\mathrm{H}), 6.80$ (d, $J=8.6 \mathrm{~Hz}, 2 \mathrm{H}, \mathrm{Ar}-\mathrm{H}), 6.72$ (d, $J=8.6 \mathrm{~Hz}, 2 \mathrm{H}$, Ar-H), 4.94 (d, $J=2.7 \mathrm{~Hz}, 1 \mathrm{H}, \mathrm{C} \underline{\mathrm{H} A r}), 4.90$ (d, $J=2.7 \mathrm{~Hz}, 1 \mathrm{H}, \mathrm{C} \underline{\mathrm{HAr}}$ ), 4.89 (s, 1H, OH), 3.08 $(\mathrm{dd}, J=10.6,2.6 \mathrm{~Hz}, 1 \mathrm{H}, \mathrm{CH}), 2.97$ (dd, $J=10.6,2.6,1 \mathrm{H}, \mathrm{CH}), 2.90-2.80(\mathrm{~m}, 2 \mathrm{H}, \mathrm{CH} H+$

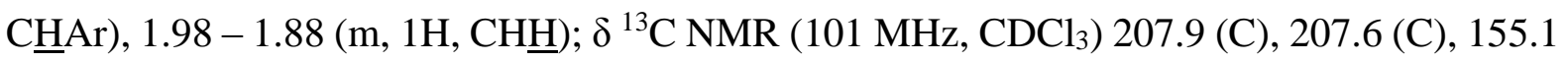
(C), $141.8(\mathrm{C}), 141.8(\mathrm{C}), 140.8(\mathrm{C}), 140.7(\mathrm{C}), 128.8(\mathrm{CH}), 127.4(\mathrm{C}), 126.7(\mathrm{CH}), 126.6(\mathrm{CH})$, $126.5(\mathrm{CH}), 125.2(\mathrm{CH}), 125.1 \mathrm{CH}), 123.8(\mathrm{CH}), 123.8(\mathrm{CH}), 115.8(\mathrm{CH}), 51.8(\mathrm{CH}), 51.2$ $(\mathrm{CH}), 50.4(\mathrm{CH}), 47.9(\mathrm{CH}), 47.6(\mathrm{CH}), 43.0\left(\mathrm{CH}_{2}\right), 1 \times$ overlapping $\mathrm{CH}$ signal; HRMS (TOF 
MS ASAP +) $m / z$ calc. for $\mathrm{C}_{26} \mathrm{H}_{19} \mathrm{O}_{3}: 379.1334[\mathrm{M}+\mathrm{H}]^{+}$; found: $379.1333 ;[\alpha]_{\mathrm{D}}^{24.1}=+16.4(\mathrm{c}$ 0.9, $\mathrm{CHCl}_{3}$ ); 85:15 e.r.; HPLC (CHIRALPAK IA, hexane/2-propanol: 90:10, flow rate: $1.0 \mathrm{~mL}$ $\min ^{-1}$, detection $\mathrm{UV} 205 \mathrm{~nm}, 25^{\circ} \mathrm{C}$ ) $\mathrm{t}_{\mathrm{R}}$ of major isomer: $27.012 \mathrm{~min}$, $\mathrm{t}_{\mathrm{R}}$ of minor isomer: 22.358 $\min$.
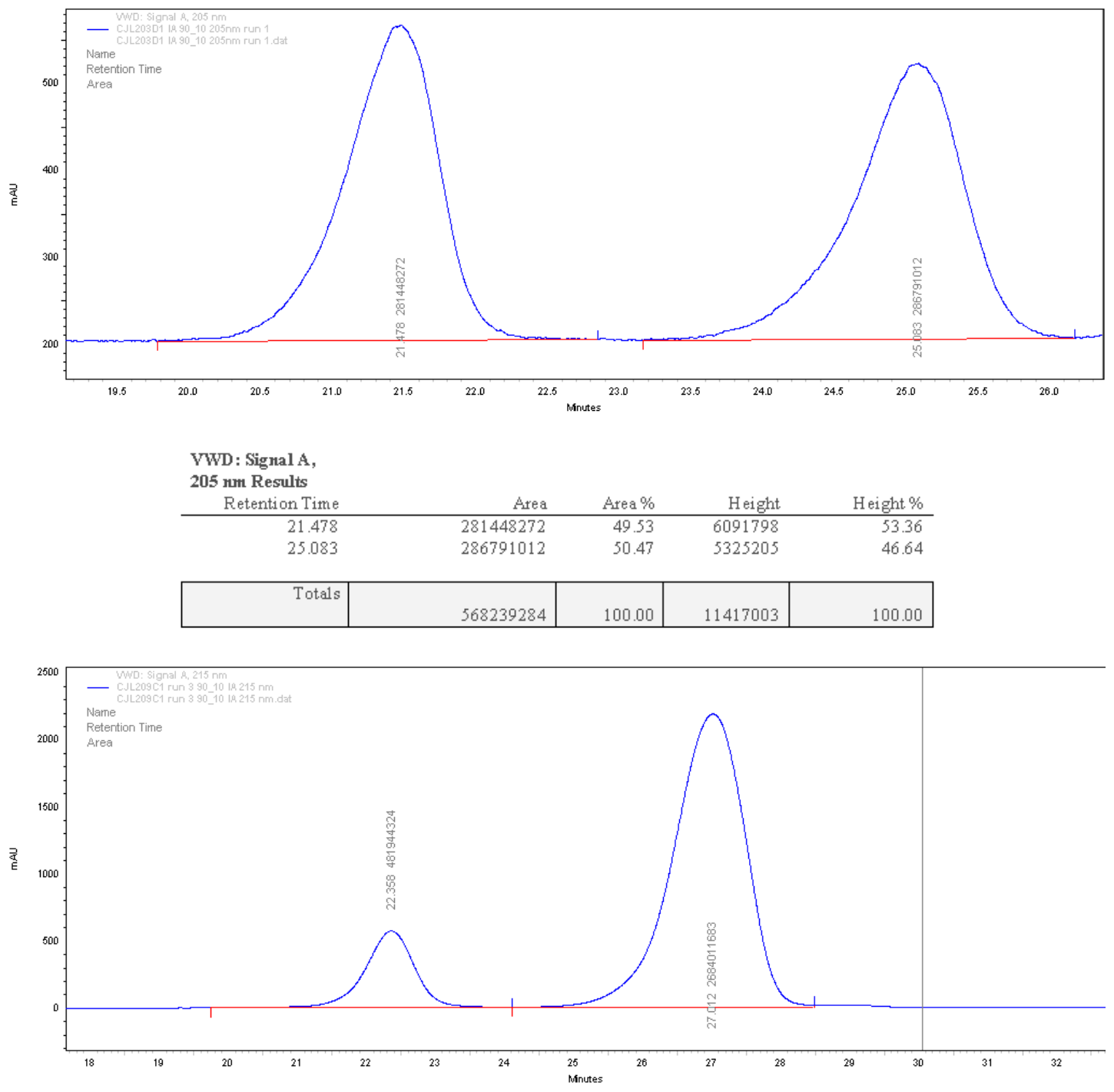

VWD : Signal A,

215 Ium Results

\begin{tabular}{r|rrrrr}
$\begin{array}{r}\text { Area } \\
\text { Retention Time }\end{array}$ & Area\% & Height & Height \% \\
\hline 22.358 & 481944324 & 15.22 & 9592696 & 20.71 \\
27.012 & 2684011683 & 84.78 & 36722983 & 79.29 \\
\hline \multicolumn{2}{|c|}{ Totals } & 3165956007 & 100.00 & 46315679 & 100.00 \\
\hline
\end{tabular}


Boronic Acid Screen Conjugate Addition products

6-(3-Methoxyphenyl)-1,4-dimethyl-2,3-diphenyl-1,4,4a,6,7,8a-hexahydro-1,4methanonaphthalene-5,8-dione $5 \mathrm{k}$

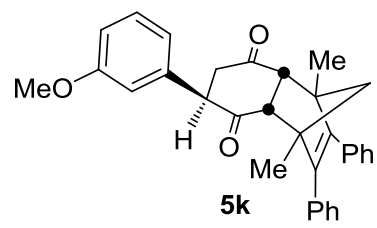

\section{Racemic procedure:}

General racemic procedure was followed. Reacting $m$-methoxybenzene boronic acid $2 \mathbf{k}$ (36.5 mg, 0.24 mmol, 2.4 equiv.) with Diels-Alder adduct 4b (35.4 mg, 0.099 mmol, 1 equiv.), $\mathrm{Pd}(\mathrm{OAc})_{2}(1.1 \mathrm{mg}, 0.005 \mathrm{mmol}, 0.05$ equiv.) and 1,10-phenanthroline (1.1 mg, $0.006 \mathrm{mmol}$, 0.06 equiv.) in DMF $(1 \mathrm{~mL})$ under at atmosphere of $\mathrm{O}_{2}$ (balloon) at $40{ }^{\circ} \mathrm{C}$ for $24 \mathrm{~h}$.. The resulting crude was purified by silica gel column chromatography (toluene/EtOAc $20: 1 \rightarrow 10: 1$ ) to yield 5k (32.3 mg, $0.070 \mathrm{mmol}, 70 \%,>20: 1$ d.r.) as a colourless fluffy solid.

\section{Enantioselective procedure:}

General enantioselective procedure A was followed. Reacting $m$-methoxybenzene boronic acid 2k (36.5 mg, 0.24 mmol, 2.4 equiv.) with with Diels-Alder adduct $4 \mathbf{b}$ (35.4 mg, 0.099 mmol, 1 equiv.), $\mathrm{Pd}(\mathrm{OAc})_{2}$ (1.1 mg, $0.005 \mathrm{mmol}, 0.05$ equiv.) and ${ }^{t} \mathrm{BuPyOx} \mathbf{L}-\mathbf{A}$ (1.2 mg, $0.006 \mathrm{mmol}$, 0.06 equiv.) in DMF $(1 \mathrm{~mL})$ under at atmosphere of $\mathrm{O}_{2}$ (balloon) at $40^{\circ} \mathrm{C}$ for $24 \mathrm{~h}$. The resulting crude was purified by silica gel column chromatography (pet. ether/EtOAc 10:1 + 10\% toluene) to yield $\mathbf{5 k}$ (27.0 mg, $0.058 \mathrm{mmol}, 58 \%,>20: 1$ d.r., $97: 3$ e.r.) as a colourless fluffy solid.

Mp: $51-54{ }^{\circ} \mathrm{C}$ (pet. ether/EtOAc); Rf: 0.2 in 10:1 pet. ether:EtOAc; $v_{\max } / \mathrm{cm}^{-1}: 2627,2870$, 1698, 1599, 1585, 1491, 1038, 741, 724, 697; ${ }^{1} \mathrm{H}$ NMR (300 MHz, $\left.\mathrm{CDCl}_{3}\right) \delta 7.29-7.22$ (m, 1H, Ar-H), 7.22 - 7.13 (m, 6H, Ar-H), 6.98 - 6.87 (m, 4H, Ar-H), 6.87 - 6.80 (m, 1H, Ar-H), $6.71-6.65$ (m, 1H, Ar-H), $6.65-6.60$ (m, 1H, Ar-H), 3.90 (dd, $J=10.0,5.2 \mathrm{~Hz}, 1 \mathrm{H}$, C 3.77 (s, 3H, OMe), 3.30 (d, $J=9.9 \mathrm{~Hz}, 1 \mathrm{H}, \mathrm{CH}), 3.22$ (d, $J=9.9 \mathrm{~Hz}, 1 \mathrm{H}, \mathrm{CH}), 3.09$ (dd, $J=$ $16.0,10.0 \mathrm{~Hz}, 1 \mathrm{H}, \mathrm{C} \underline{\mathrm{H}} \mathrm{H}), 2.82(\mathrm{dd}, J=16.0,5.2 \mathrm{~Hz}, 1 \mathrm{H}, \mathrm{CH} \underline{\mathrm{H}}), 1.84(\mathrm{~d}, J=8.5 \mathrm{~Hz}, 1 \mathrm{H}, \mathrm{C} \underline{\mathrm{H}})$, $1.62\left(\mathrm{~s}, 3 \mathrm{H}, \mathrm{CH}_{3}\right), 1.59$ (d, $\left.J=8.5 \mathrm{~Hz}, 1 \mathrm{H}, \mathrm{CH} \underline{\mathrm{H}}\right), 1.56\left(\mathrm{~s}, 3 \mathrm{H}, \mathrm{CH}_{3}\right) ;{ }^{13} \mathrm{C} \mathrm{NMR}(75 \mathrm{MHz}$, $\left.\mathrm{CDCl}_{3}\right) 208.4$ (C), 208.3 (C), 160.0 (C), 147.3 (C), 146.8 (C), 137.7 (C), 135.6 (C), 135.4 (C), $130.0(\mathrm{CH}), 129.3(\mathrm{CH}), 129.2(\mathrm{CH}), 128.3(\mathrm{CH}), 128.1(\mathrm{CH}), 127.1(\mathrm{CH}), 120.2(\mathrm{CH}), 114.0$ $(\mathrm{CH}), 113.2(\mathrm{CH}), 66.0\left(\mathrm{CH}_{2}\right), 60.1(\mathrm{CH}), 59.0(\mathrm{CH}), 58.1(2 \times \mathrm{C}), 55.3\left(\mathrm{CH}_{3}\right), 52.9(\mathrm{CH}), 45.1$ $\left(\mathrm{CH}_{2}\right), 19.1\left(\mathrm{CH}_{3}\right), 18.8\left(\mathrm{CH}_{3}\right)$ with 1 overlapping aromatic $\mathrm{CH}$ signal; $\delta$ HRMS (TOF MS ASAP+) $m / z$ calc. for $\mathrm{C}_{32} \mathrm{H}_{29} \mathrm{O}_{3}: 461.2117[\mathrm{M}-\mathrm{H}]^{+}$; found: 461.2110; $[\alpha]_{\mathrm{D}}^{19.1}=+20.3(c 1.63$, 
$\mathrm{CHCl}_{3}$ ); 97:3 e.r.; HPLC (CHIRALPAK IA, hexane/2-propanol: 95:5, flow rate: $1.0 \mathrm{~mL} \mathrm{~min}^{-}$ ${ }^{1}$, detection UV $215 \mathrm{~nm}, 25^{\circ} \mathrm{C}$ ) $\mathrm{t}_{\mathrm{R}}$ of major isomer: $14.487 \mathrm{~min}, \mathrm{t}_{\mathrm{R}}$ of minor isomer: 16.582 $\min$.

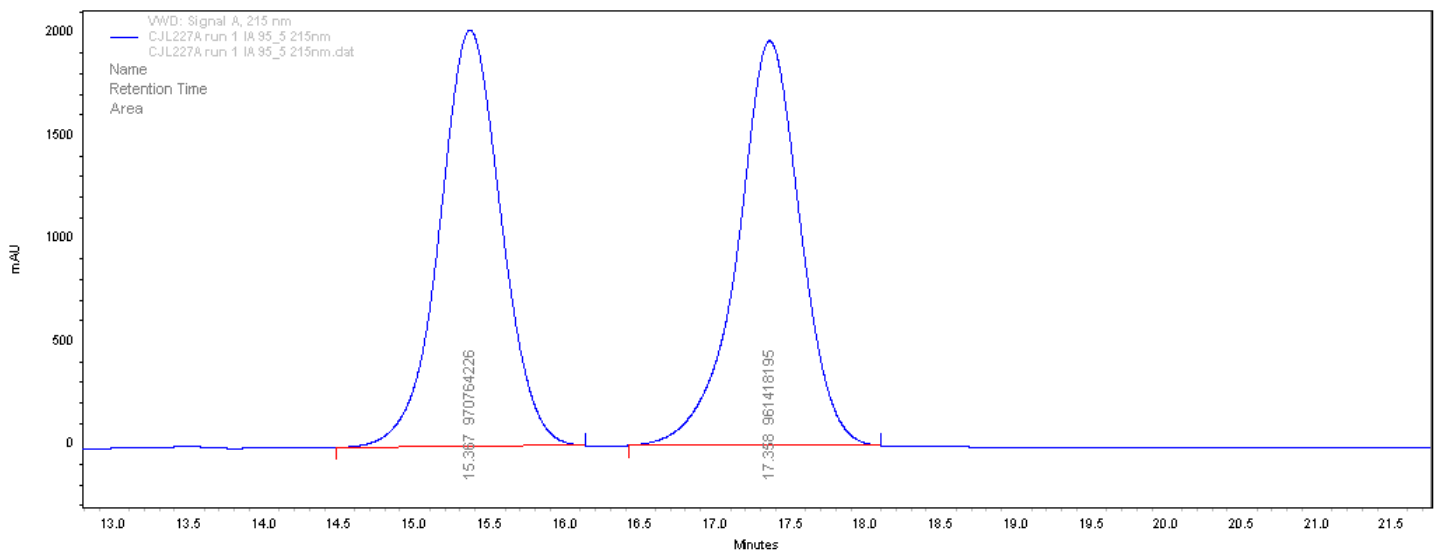

VWD : Signal A,

$215 \mathrm{~nm}$ Results

Retention Tim

$\begin{array}{lllll}15.367 & 970764226 & 50.24 & 33936076 & 50.70 \\ 17.358 & 961418195 & 49.76 & 32995594 & 49.30\end{array}$

\begin{tabular}{|r|r|r|r|r|}
\hline Totals & 1932182421 & 100.00 & 66931670 & 100.00 \\
\hline
\end{tabular}

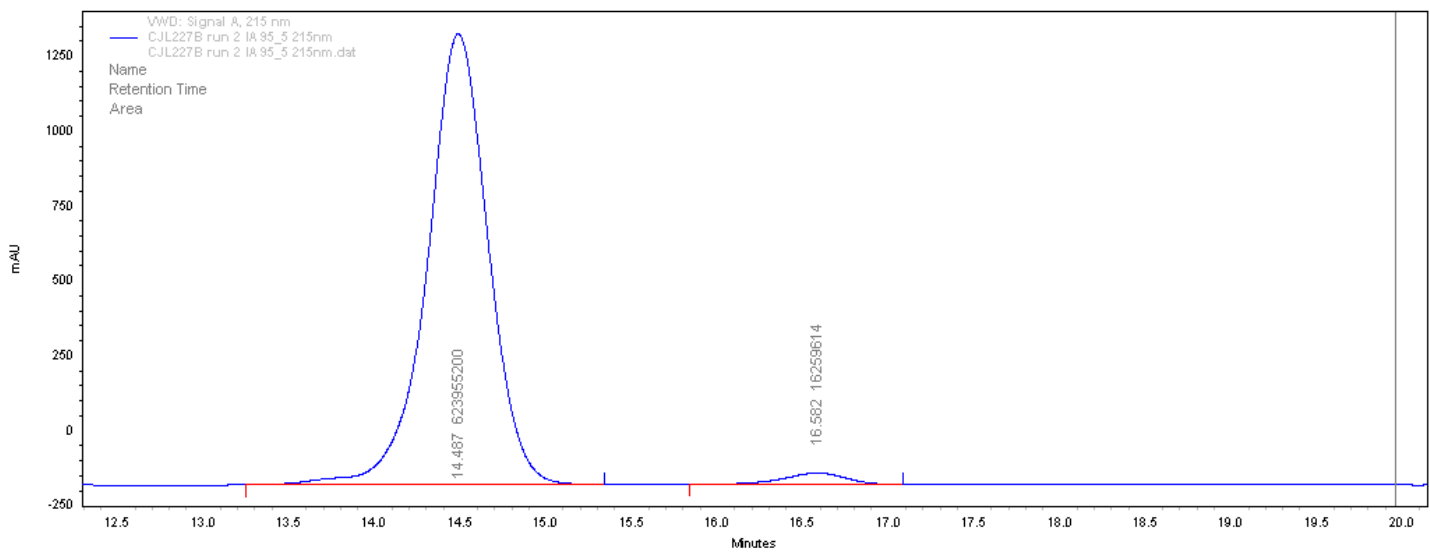

VWD: Signal A,

215 um Results

Retention Time

\begin{tabular}{rrrrr} 
& Area & Area $\%$ & Height & He1ght $\%$ \\
\hline 14.487 & 623955200 & 97.46 & 25252947 & 97.53 \\
16.582 & 16259614 & 2.54 & 639770 & 2.47
\end{tabular}

Totals 
6-(2-Methoxyphenyl)-1,4-dimethyl-2,3-diphenyl-1,4,4a,6,7,8a-hexahydro-1,4methanonaphthalene-5,8-dione $5 \mathrm{l}$

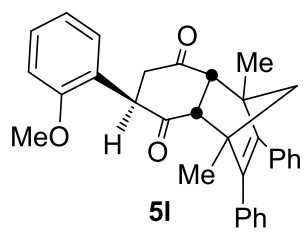

\section{Racemic procedure:}

General racemic procedure was used with modifications. Reacting $o$-methoxyphenylboronic acid 21 (36.4.0 mg, 0.24 mmol, 2.4 equiv.), Diels-Alder adduct 4b (35.4 mg, 0.099 mmol, 1.0 equiv.), $\mathrm{Pd}(\mathrm{OAc})_{2}(1.1 \mathrm{mg}, 0.005 \mathrm{mmol}, 0.05$ equiv.) and 1,10-phenanthroline (1.1 mg, 0.006 mmol, 0.06 equiv.) in DMF ( $1 \mathrm{~mL})$ under at atmosphere of $\mathrm{O}_{2}$ (balloon) at $50{ }^{\circ} \mathrm{C}$ for $24 \mathrm{~h}$. The resulting crude was purified by silica gel column chromatography (pet. ether/EtOAc 10:1 + $10 \%$ toluene) to yield $\mathbf{5 l}(23.1 \mathrm{mg}, 0.05 \mathrm{mmol}, 50 \%,>20: 1$ d.r. $)$ as a white solid.

\section{Enantioselective procedure:}

General enantioselective procedure B was followed. Reacting $o$-methoxyphenylboronic acid 21 (3.4.0 mg, 0.24 mmol, 2.4 equiv.), Diels-Alder adduct $4 \mathbf{b}$ (35.4 mg, 0.099 mmol, 1.0 equiv.), $\mathrm{Pd}(\mathrm{OAc})_{2}\left(2.2 \mathrm{mg}, 0.010 \mathrm{mmol}, 0.10\right.$ equiv.) and ${ }^{t} \mathrm{BuPyOx} \mathbf{L}-\mathbf{A}(2.1 \mathrm{mg}, 0.011 \mathrm{mmol}, 0.11$ equiv.) in DMF (1 mL) under at atmosphere of $\mathrm{O}_{2}$ (balloon) at $40{ }^{\circ} \mathrm{C}$ for $72 \mathrm{~h}$. The resulting crude was purified by silica gel column chromatography (pet. ether/EtOAc 10:1 + 10\% toluene) to yield $5 \mathbf{l}$ (21.1 mg, $0.046 \mathrm{mmol}, 46 \%,>20: 1$ d.r., 92:8 e.r.) as a white powder.

Mp: $135-137{ }^{\circ} \mathrm{C}$; $\mathrm{R}_{\mathrm{f}}: 0.2$ in 10:1 pet. ether:EtOAc; vmax/ $\mathrm{cm}^{-1}: 2924,1699,1600,1494,1462$, 1440, 1246, 1030, 752, 743, 698; ${ }^{1} \mathrm{H}$ NMR (300 MHz, $\left.\mathrm{CDCl}_{3}\right) 7.24$ - 7.17 (m, 4H, Ar-H), 7.13 $-7.03(\mathrm{~m}, 5 \mathrm{H}, \mathrm{Ar}-\mathrm{H}), 7.00$ (dd, $J=7.5,1.8 \mathrm{~Hz}, 1 \mathrm{H}, \mathrm{Ar}-\mathrm{H}), 6.92(\mathrm{td}, J=7.4,1.1 \mathrm{~Hz}, 1 \mathrm{H}, \mathrm{Ar}-$ H), $6.88-6.81(\mathrm{~m}, 3 \mathrm{H}, \mathrm{Ar}-\mathrm{H}), 4.14(\mathrm{dd}, J=11.8,6.0 \mathrm{~Hz}, 1 \mathrm{H}, \mathrm{CHH}), 3.57$ (s, 3H, $\left.\mathrm{OCH}_{3}\right), 3.40$ $(\mathrm{d}, J=9.9 \mathrm{~Hz}, 1 \mathrm{H}, \mathrm{CH}), 3.27(\mathrm{~d}, J=9.9,1 \mathrm{H}, \mathrm{CH}), 3.06(\mathrm{dd}, J=16.1,11.8 \mathrm{~Hz}, 1 \mathrm{H}), 2.72(\mathrm{dd}$, $J=16.1,6.0 \mathrm{~Hz}, 1 \mathrm{H}), 1.86(\mathrm{~d}, J=8.4 \mathrm{~Hz}, 1 \mathrm{H}, \mathrm{CH} \underline{\mathrm{H}}), 1.68\left(\mathrm{~s}, 3 \mathrm{H}, \mathrm{CH}_{3}\right), 1.64(\mathrm{~d}, J=8.4 \mathrm{~Hz}$,

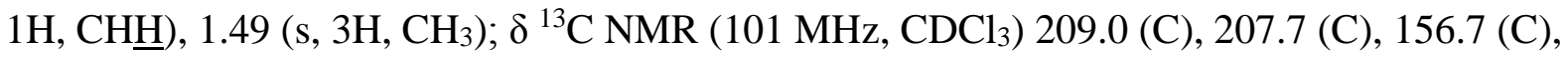
147.5 (C), $146.5(\mathrm{C}), 136.0(\mathrm{C}), 135.3(\mathrm{C}), 130.2(\mathrm{C}), 129.5(\mathrm{CH}), 129.2(\mathrm{CH}), 129.0(\mathrm{CH})$, $128.2(\mathrm{CH}), 128.0(\mathrm{CH}), 127.0(\mathrm{CH}), 126.8(\mathrm{CH}), 126.4(\mathrm{C}), 121.0(\mathrm{CH}), 111.0(\mathrm{CH}), 66.2$ $\left(\mathrm{CH}_{2}\right), 60.7(\mathrm{CH}), 60.1(\mathrm{CH}), 58.7(\mathrm{C}), 57.7(\mathrm{C}), 55.0\left(\mathrm{CH}_{3}\right), 48.6(\mathrm{CH}), 46.0\left(\mathrm{CH}_{2}\right), 19.4$ $\left(\mathrm{CH}_{3}\right), 18.4\left(\mathrm{CH}_{3}\right) ; \delta$ HRMS (FTMS + p NSI) $\mathrm{m} / z$ calc. for $\mathrm{C}_{32} \mathrm{H}_{30} \mathrm{O}_{3} \mathrm{NH}_{4}: 480.2533$ $\left[\mathrm{M}+\mathrm{NH}_{4}{ }^{+}\right]+$; found: $480.2528 ;[\alpha]_{\mathrm{D}}^{21.0}=-12.0\left(\mathrm{c} 0.5, \mathrm{CHCl}_{3}\right) ; 92: 8$ e.r.; HPLC $($ CHIRALPAK 
IA, hexane/2-propanol: 95:5, flow rate: $1.0 \mathrm{~mL} \mathrm{~min}^{-1}$, detection UV $215 \mathrm{~nm}, 25^{\circ} \mathrm{C}$ ) $\mathrm{t}_{\mathrm{R}}$ of major isomer: $15.148 \mathrm{~min}, \mathrm{t}_{\mathrm{R}}$ of minor isomer: $12.303 \mathrm{~min}$.

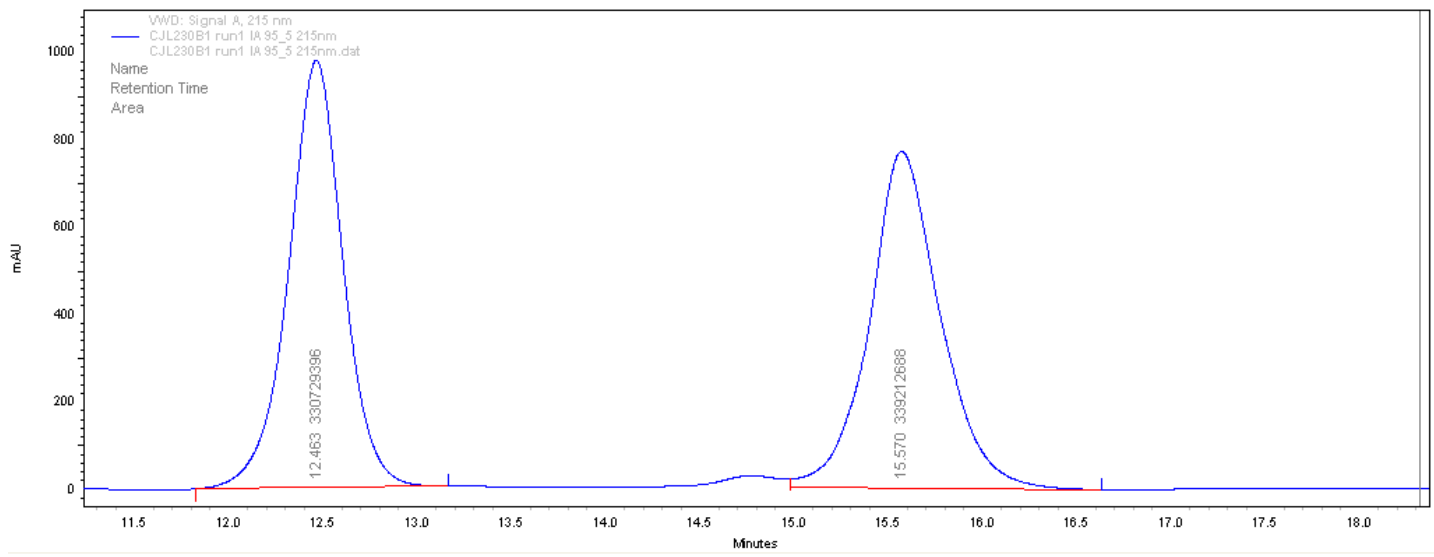

VWD : Signal A,

215 num Results

Retention Time

\begin{tabular}{rrrrr} 
Area & Area \% & Height & Height \% \\
\hline 12.463 & 330729396 & 49.37 & 16420198 & 55.92 \\
15.570 & 339212688 & 50.63 & 12943778 & 44.08
\end{tabular}

\begin{tabular}{|r|r|r|r|r|}
\hline Totals & 669942084 & 100.00 & 29363976 & 100.00 \\
\hline
\end{tabular}

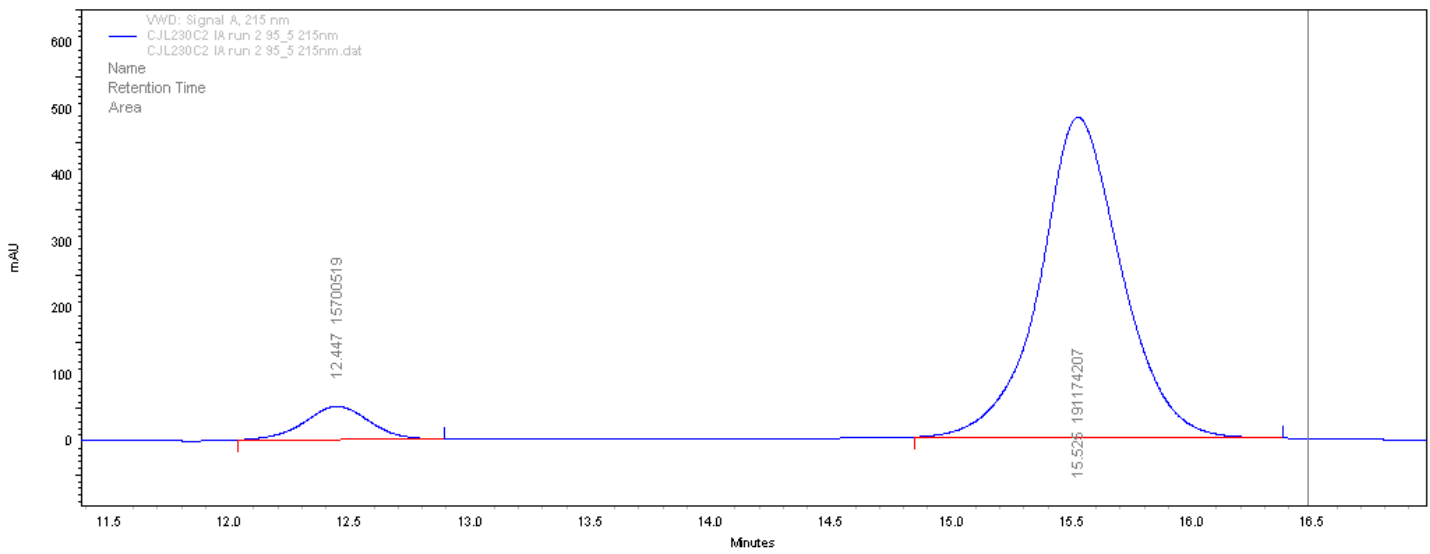

VWD : Signal A,

215 um Results

Retention Time

12.447
15.525

$\begin{array}{rrr}\text { Area } & \text { Area \% } & \text { Height } \\ 15700519 & 7.59 & 837338\end{array}$

Height \%

Totals

191174207

$92.41 \quad 8091628$

90.62

\begin{tabular}{|r|r|r|r|r|}
\hline Totals & 206874726 & 100.00 & 8928966 & 100.00 \\
\hline
\end{tabular}




\section{6-(4-Hydroxyphenyl)-1,4-dimethyl-2,3-diphenyl-1,4,4a,6,7,8a-hexahydro-1,4- methanonaphthalene-5,8-dione $5 \mathrm{~m}$}

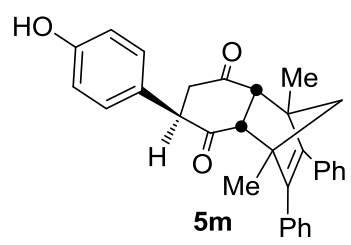

\section{Racemic procedure:}

General racemic procedure was followed. Reacting $P$-hydroxylbenzene boronic acid $\mathbf{2 m}(33.1$ mg, 0.24 mmol, 2.4 equiv.), Diels-Alder adduct $4 \mathbf{b}$ (35.4 mg, 0.099 mmol, 1 equiv.), $\mathrm{Pd}(\mathrm{OAc})_{2}$ (1.1 mg, $0.005 \mathrm{mmol}, 0.05$ equiv.) and 1,10-phenanthroline (1.1 mg, $0.006 \mathrm{mmol}, 0.06$ equiv.) in DMF (1 mL) under an atmosphere of $\mathrm{O}_{2}$ (balloon) at $40{ }^{\circ} \mathrm{C}$ for $24 \mathrm{~h}$. The resulting crude was purified by silica gel column chromatography (pet. ether /EtOAc 10:1 $\rightarrow 3: 1)$ to $\mathbf{5 m}(33.7 \mathrm{mg}$, $0.075 \mathrm{mmol}, 75 \%,>20: 1$ d.r.) as a colourless oil.

\section{Enantioselective procedure:}

General enantioselective procedure A was followed. Reacting $P$-hydroxylbenzene boronic acid 2m (33.1 mg, 0.24 mmol, 2.4 equiv.), Diels-Alder adduct 4b (35.4 mg, 0.099 mmol, 1 equiv.) $\mathrm{Pd}(\mathrm{OAc})_{2}$ (1.1 mg, $0.005 \mathrm{mmol}, 0.05$ equiv.) and ${ }^{t} \mathrm{BuPyOx}$ L-A (1.2 mg, $0.006 \mathrm{mmol}, 0.06$ equiv.) in DMF (1 mL) under atmosphere of $\mathrm{O}_{2}$ (balloon) at $40{ }^{\circ} \mathrm{C}$ for $72 \mathrm{~h}$.. The resulting crude was purified by silica gel column chromatography (pet. ether/EtOAc 10:1 $\rightarrow 3: 1$ ) to yield $\mathbf{5 m}$ (29.1 mg, $0.065 \mathrm{mmol}, 65 \%$, >20:1 d.r., 98:2 e.r.) as a colourless oil.

$\mathrm{R}_{\mathrm{f}}$ : 0.3 in 3:1 pet. ether:EtOAc; $v_{\max } / \mathrm{cm}^{-1}: 3366,2972,2870,1694,1614,1597,1515,822$, 775, 698); ${ }^{1} \mathrm{H}$ NMR $\left(300 \mathrm{MHz}, \mathrm{CDCl}_{3}\right) \delta 7.22$ - 7.11 (m, 6H, Ar-H), 6.96 - 6.85 (m, 6H, ArH), 6.77 - 6.70 (m, 2H, Ar-H), 5.87 (s, 1H, OH), 3.88 (dd, J = 9.6, 5.2 Hz, 1H, CㅌAㄱ), 3.29 (d, $J=9.9 \mathrm{~Hz}, 1 \mathrm{H}, \mathrm{CH}), 3.21(\mathrm{~d}, J=9.9 \mathrm{~Hz}, 1 \mathrm{H}, \mathrm{CH}), 3.08$ (dd, $J=16.0,9.6 \mathrm{~Hz}, 1 \mathrm{H}, \mathrm{CHH})$, $2.83(\mathrm{dd}, J=16.0,5.2 \mathrm{~Hz}, 1 \mathrm{H}, \mathrm{CH} \underline{\mathrm{H}}), 1.84(\mathrm{~d}, J=8.5 \mathrm{~Hz}, 1 \mathrm{H}, \mathrm{C} \underline{\mathrm{HH}}), 1.61\left(\mathrm{~s}, 3 \mathrm{H}, \mathrm{CH}_{3}\right), 1.57$ (s, 4H, $\mathrm{CH}_{3}$ and an overlapping $\mathrm{CH} \underline{\mathrm{H}}$ signal); ${ }^{13} \mathrm{C} \mathrm{NMR}\left(75 \mathrm{MHz}, \mathrm{CDCl}_{3}\right) \delta 209.7$ (C), 209.2 (C), $155.5(\mathrm{C}), 147.1(\mathrm{C}), 146.9(\mathrm{C}), 135.5(\mathrm{C}), 135.4(\mathrm{C}), 129.3(\mathrm{CH}), 129.3(\mathrm{CH}), 129.2(\mathrm{CH})$, $128.3(\mathrm{CH}), 128.2(\mathrm{CH}), 127.8(\mathrm{C}), 127.1(\mathrm{CH}), 116.0(\mathrm{CH}), 66.1\left(\mathrm{CH}_{2}\right), 60.2(\mathrm{CH}), 59.0(\mathrm{CH})$, $58.2(\mathrm{C}), 58.1(\mathrm{C}), 52.2(\mathrm{CH}), 45.2\left(\mathrm{CH}_{2}\right), 19.0\left(\mathrm{CH}_{3}\right), 18.8\left(\mathrm{CH}_{3}\right)$, with 1 overlapping aromatic $\mathrm{CH}$ signals; HRMS (FTMS + p APCI corona) $m / z$ calc. for $\mathrm{C}_{31} \mathrm{H}_{27} \mathrm{O}_{3}: 447.1955[\mathrm{M}-\mathrm{H}]^{+}$; found: 477.1945; $[\alpha]_{\mathrm{D}}^{23.5}=+3.6\left(c \quad 0.3, \mathrm{CHCl}_{3}\right)$; 98:2 e.r.; HPLC (CHIRALPAK IA, hexane/2propanol: 97:3, flow rate: $1.0 \mathrm{~mL} \mathrm{~min}^{-1}$, detection $\mathrm{UV} 215 \mathrm{~nm}, 25^{\circ} \mathrm{C}$ ) $\mathrm{t}_{\mathrm{R}}$ of major isomer: $20.535 \mathrm{~min}, \mathrm{t}_{\mathrm{R}}$ of minor isomer: $36.750 \mathrm{~min}$. 


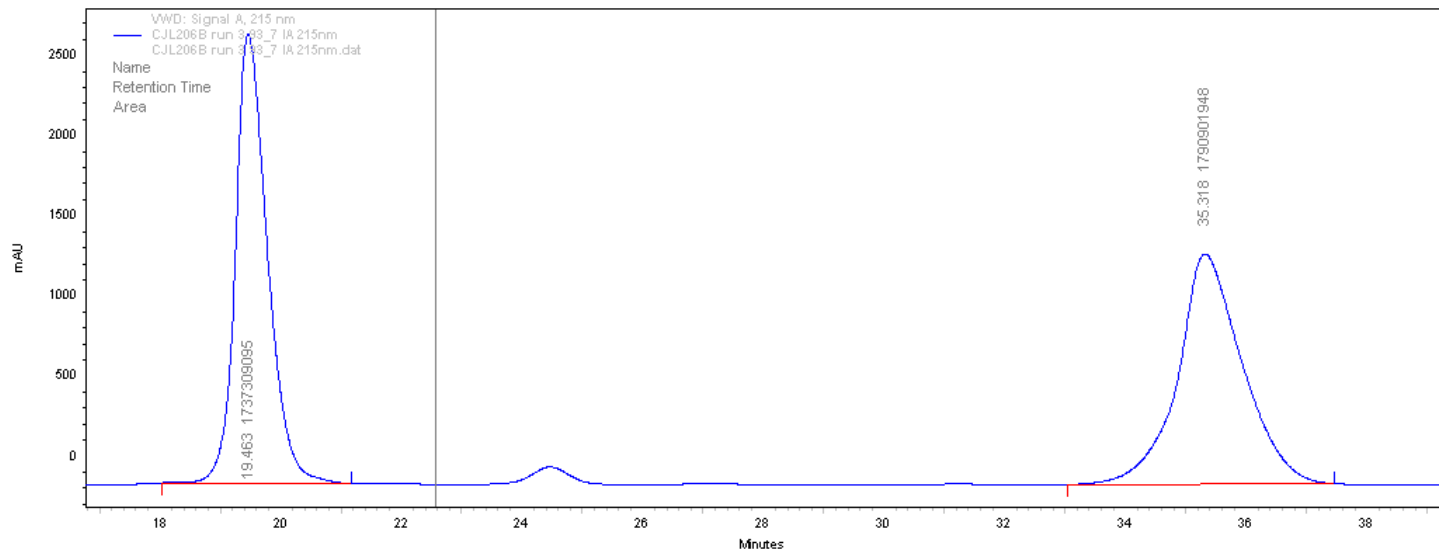

VWD : Signal A,

215 vim Results

\begin{tabular}{rrrrr} 
Retention Time & Area & Area\% & Height & Height\% \\
\hline 19.463 & 1737309095 & 49.24 & 46980970 & 66.11 \\
35.318 & 1790901948 & 50.76 & 24087665 & 33.89
\end{tabular}

\begin{tabular}{|r|r|r|r|r|}
\hline Totals & 3528211043 & 100.00 & 71068635 & 100.00 \\
\hline
\end{tabular}

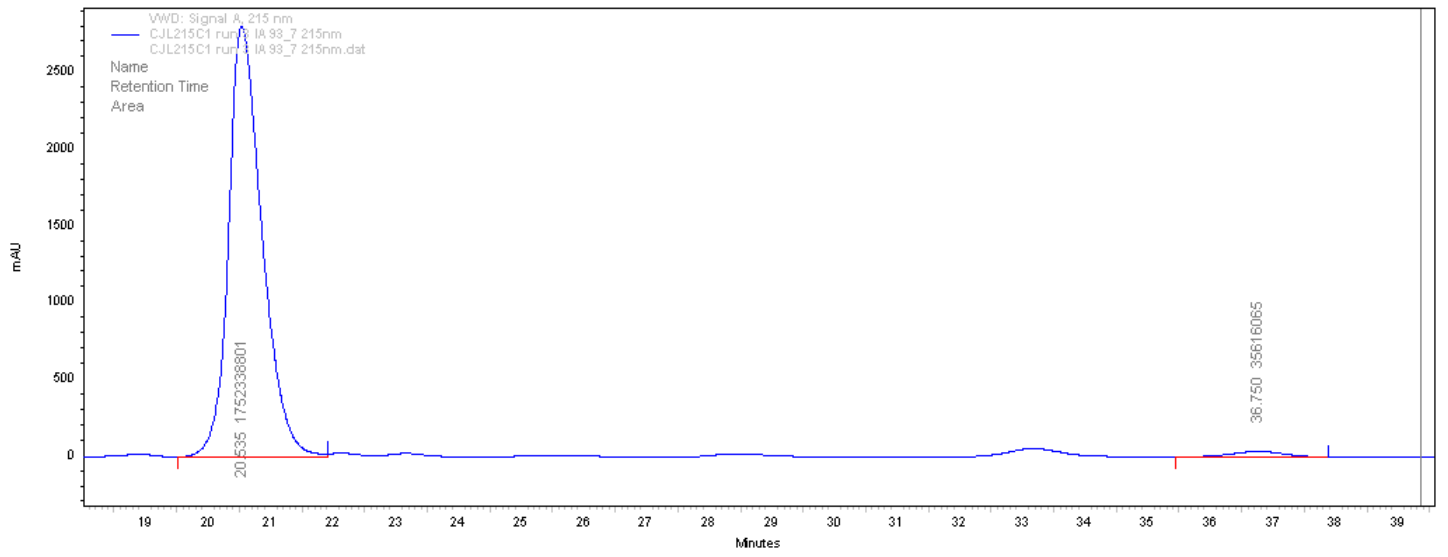

VWD : Signal A,

215 um Results

Retention Time

$\begin{array}{rrrrr}20.535 & 1752338801 & 98.01 & 47104916 & 98.71 \\ 36.750 & 35616065 & 1.99 & 614149 & 1.29\end{array}$

Totals 
1,4-Dimethyl-2,3,6-triphenyl-1,4,4a,6,7,8a-hexahydro-1,4-methanonaphthalene-5,8dione 5n

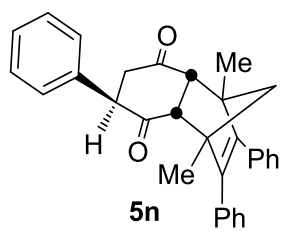

\section{Racemic procedure:}

General racemic procedure was followed with modificiations. Reacting phenylboronic acid $\mathbf{2 n}$ (29.3 mg, 0.24 mmol, 2.4 equiv.) with Diels-Alder adduct 4b (35.4 mg, 0.099 mmol, 1.0 equiv.), $\mathrm{Pd}(\mathrm{OAc})_{2}(1.1 \mathrm{mg}, 0.005 \mathrm{mmol}, 0.05$ equiv.) and 1,10-phenanthroline (1.1 mg, 0.006 mmol, 0.06 equiv.) in DMF (0.5 mL) under at atmosphere of $\mathrm{O}_{2}$ (balloon) at $40{ }^{\circ} \mathrm{C}$ for $24 \mathrm{~h}$. The resulting crude was purified by silica gel column chromatography (toluene/EtOAc 10:1+ $10 \%$ toluene) to yield $\mathbf{5 n}(29.9 \mathrm{mg}, 0.069 \mathrm{mmol}, 69 \%,>20: 1 \mathrm{~d} . r$. $)$ as a fluffy white solid.

\section{Enantioselective procedure:}

General enantioselective procedure B was followed. Reacting phenylboronic acid 2n $(29.3 \mathrm{mg}$, $0.24 \mathrm{mmol}, 2.4$ equiv.), Diels-Alder adduct $4 \mathbf{b}$ (35.4 mg, $0.099 \mathrm{mmol}, 1.0$ equiv.), $\mathrm{Pd}(\mathrm{OAc})_{2}$ (2.2 mg, $0.010 \mathrm{mmol}, 0.10$ equiv.) and ${ }^{t} \mathrm{BuPyOx}$ L-A (2.1 mg, $0.011 \mathrm{mmol}, 0.11$ equiv.) in DMF $(0.5 \mathrm{~mL})$ under at atmosphere of $\mathrm{O}_{2}$ (balloon) at $40{ }^{\circ} \mathrm{C}$ for $72 \mathrm{~h}$.. The resulting crude was purified by silica gel column chromatography (pet. ether/EtOAc 10:1 $+10 \%$ toluene) to yield 5n (36.2 mg, $0.083 \mathrm{mmol}, 83 \%,>20: 1$ d.r., $97: 3$ e.r.) as a white fluffy crystalline solid.

Mp: $56-58{ }^{\circ} \mathrm{C}$; $\mathrm{R}_{\mathrm{f}}: 0.2$ in 10:1 pet. ether:EtOAc; $v_{\max } / \mathrm{cm}^{-1}: 3025,2933,2867,1700,100$, 1574, 1454, 1041, 800, 775, 693; ${ }^{1} \mathrm{H}$ NMR (300 MHz, $\left.\mathrm{CDCl}_{3}\right) 7.39$ - 7.28 (m, 3H, Ar-H), 7.24 - 7.12 (m, 6H, Ar-H), 7.11 - 7.04 (m, 2H, Ar-H), 6.98 - 6.85 (m, 4H, Ar-H), 3.94 (dd, J =

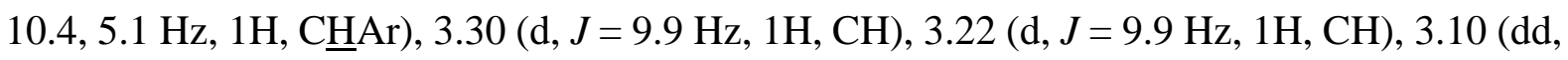
$J=16.0,10.4,1 \mathrm{H}, \underline{\mathrm{CH}}), 2.82(\mathrm{dd}, J=16.0,5.1 \mathrm{~Hz}, 1 \mathrm{H}, \mathrm{CH} \underline{\mathrm{H}}), 1.84(\mathrm{~d}, J=8.5 \mathrm{~Hz}, 1 \mathrm{H}, \mathrm{C} \underline{\mathrm{H}})$, $1.63\left(\mathrm{~s}, 3 \mathrm{H}, \mathrm{CH}_{3}\right), 1.59$ (d, $\left.J=8.5 \mathrm{~Hz}, 1 \mathrm{H}, \mathrm{CH} \underline{\mathrm{H}}\right), 1.55\left(\mathrm{~s}, 3 \mathrm{H}, \mathrm{CH}_{3}\right) ; \delta{ }^{13} \mathrm{C} \mathrm{NMR}(101 \mathrm{MHz}$, $\mathrm{CDCl}_{3}$ ) 208.4 (broad, C), 147.4 (C), 146.9 (C), 136.3 (broad, C), 135.7 (C), 135.5 (C), 129.4 $(\mathrm{CH}), 129.3(\mathrm{CH}), 129.0(\mathrm{CH}), 128.3(\mathrm{CH}), 128.2(\mathrm{CH}), 128.1(\mathrm{CH}), 127.8(\mathrm{CH}), 127.1(\mathrm{CH})$, $66.2\left(\mathrm{CH}_{2}\right), 60.3(\mathrm{CH}), 59.2(\mathrm{CH}), 58.2(\mathrm{C}), 58.1(\mathrm{C}), 52.9(\mathrm{CH}), 45.3\left(\mathrm{CH}_{2}\right), 19.1\left(\mathrm{CH}_{3}\right), 18.7$ $\left(\mathrm{CH}_{3}\right), 1 \times$ overlapping $\mathrm{C}$ signals $1 \times$ overlapping $\mathrm{CH}$ signal; HRMS (TOF MS ASAP + ) $\mathrm{m} / \mathrm{z}$ calc. for $\mathrm{C}_{31} \mathrm{H}_{29} \mathrm{O}_{2}$ : $433.2168[\mathrm{M}+\mathrm{H}]^{+}$; found: 433.2164; $[\alpha]_{\mathrm{D}}^{20.4}=+10.1\left(\right.$ c 1.6, $\left.\mathrm{CHCl}_{3}\right) ; 97: 3$ e.r.; HPLC (CHIRALPAK IA, hexane/2-propanol: 95:5, flow rate: $1.0 \mathrm{~mL} \mathrm{~min}^{-1}$, detection UV $215 \mathrm{~nm}, 25^{\circ} \mathrm{C}$ ) $\mathrm{t}_{\mathrm{R}}$ of major isomer: $11.583 \mathrm{~min}, \mathrm{t}_{\mathrm{R}}$ of minor isomer: $9.343 \mathrm{~min}$. 


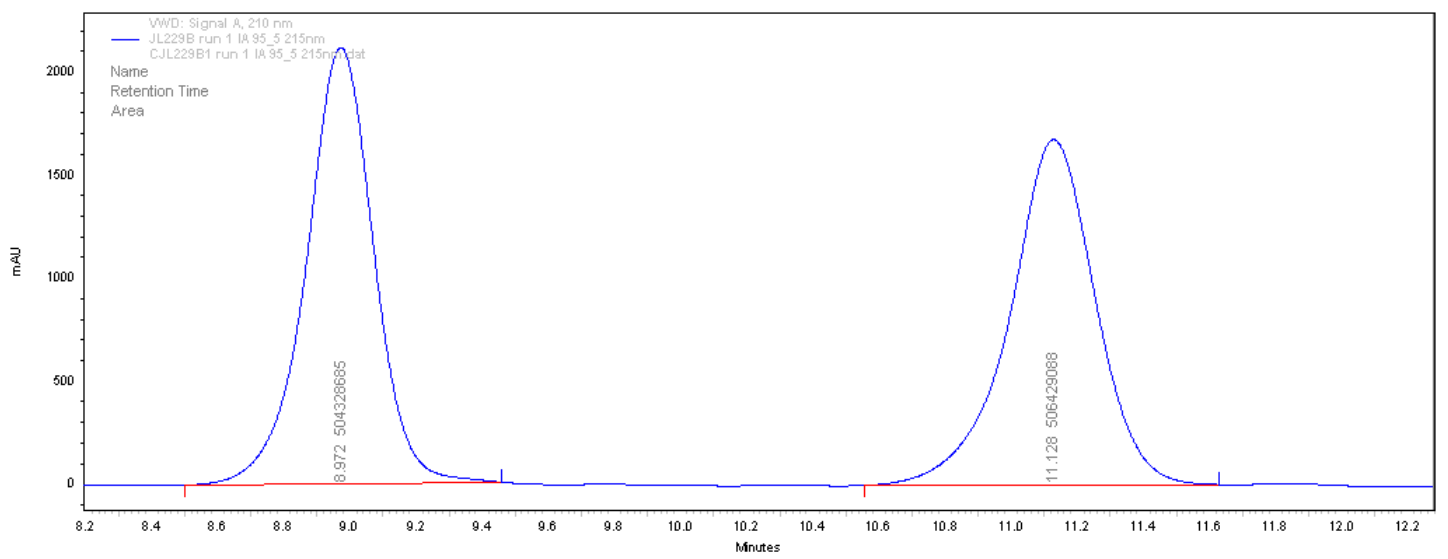

VWD: Signal A,

210 um Results

Retention Time

\begin{tabular}{rrrrr} 
Axea & Axea $\%$ & He1ght & Height $\%$ \\
\hline 8.972 & 504328685 & 49.90 & 35478001 & 55.79
\end{tabular}

11.128

506429088

$50.10 \quad 2811729$

4421

Totals

1010757773

$100.00 \quad 63595299$

100.00

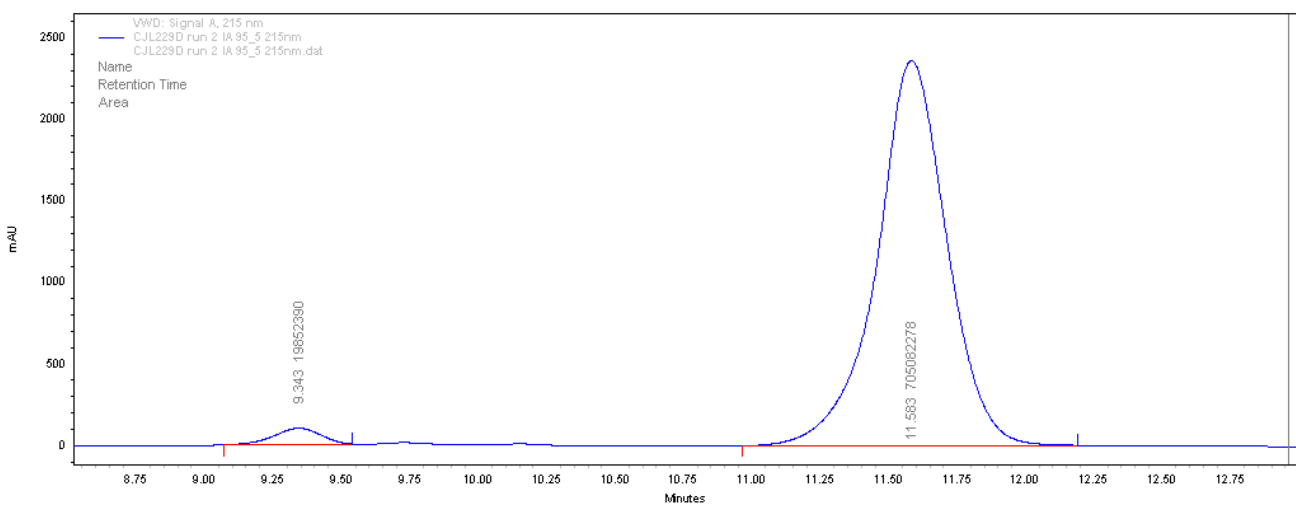

VWD : Signal A,

$215 \mathrm{~nm}$ Results

\begin{tabular}{r|rrrrr|}
\multicolumn{1}{r}{ Retention Time } & Area & Area $\%$ & Height & Height \% \\
\hline 9.343 & 19852390 & 2.74 & 1661757 & 4.03 \\
11.583 & 705082278 & 97.26 & 39578362 & 95.97 \\
\hline \multicolumn{2}{|c|}{ Totals } & 724934668 & 100.00 & 41240119 & 100.00 \\
\hline
\end{tabular}


1,4-Dimethyl-2,3-diphenyl-6-(p-tolyl)-1,4,4a,6,7,8a-hexahydro-1,4-methanonaphthalene5,8-dione 50

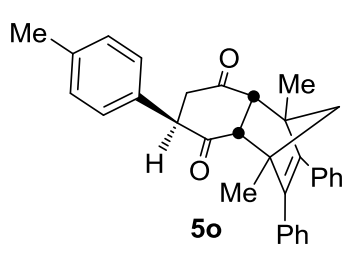

\section{Racemic procedure:}

General racemic procedure was followed. Reacting $p$-tolylbenzene boronic acid 20 (32.6 mg, 0.24 mmol, 2.4 equiv.), Diels-Alder adduct $4 \mathbf{b}$ (35.4 mg, 0.099 mmol, 1 equiv.), $\mathrm{Pd}(\mathrm{OAc})_{2}$ (1.1 $\mathrm{mg}, 0.005 \mathrm{mmol}, 0.05$ equiv.) and 1,10-phenanthroline (1.1 mg, $0.006 \mathrm{mmol}, 0.06$ equiv.) in DMF (1 mL) under an atmosphere of oxygen (balloon) at $40{ }^{\circ} \mathrm{C}$ for $24 \mathrm{~h}$.. The resulting crude was purified by silica gel column chromatography (toluene/EtOAc $20: 1 \rightarrow 10: 1$ ) to yield $\mathbf{5 0}$ (40.3 mg, coeluted with starting material $\mathbf{4 b},>20: 1$ d.r.) as a pale-yellow oil.

\section{Enantioselective procedure:}

General enantioselective procedure B was followed. Reacting $p$-tolylbenzene boronic acid 20 (32.6 mg, 0.24 mmol, 2.4 equiv.) with Diels-Alder adduct 4b (35.4 mg, 0.099 mmol, 1 equiv.), $\mathrm{Pd}(\mathrm{OAc})_{2}\left(2.2 \mathrm{mg}, 0.010 \mathrm{mmol}, 0.10\right.$ equiv.) and ${ }^{t} \mathrm{BuPyOx} \mathbf{L}-\mathbf{A}(2.1 \mathrm{mg}, 0.010 \mathrm{mmol}, 0.10$ equiv.) in DMF ( $1 \mathrm{~mL}$ ) under an atmosphere of $\mathrm{O}_{2}$ (balloon) at $40{ }^{\circ} \mathrm{C}$ for $72 \mathrm{~h}$.. The resulting crude was purified by silica gel column chromatography (pet. ether/EtOAc 10:1 $\rightarrow$ 3:1) to yield 5o (36.0 mg, $0.080 \mathrm{mmol}, 81 \%$, >20:1 d.r., 97:3 e.r.) as a colourless oil.

$\mathrm{R}_{\mathrm{f}}$ : 0.2 in 10:1 pet. ether:EtOAc; $v_{\max } / \mathrm{cm}^{-1}: 2925,2869,1699,1599,1574,1488,1442,817$, 743, 698; ${ }^{1} \mathrm{H}$ NMR (300 MHz, $\left.\mathrm{CDCl}_{3}\right) \delta 7.24$ - 7.12 (m, 8H, Ar-H), 7.02 - 6.86 (m, 6H, Ar-H), $3.91(\mathrm{dd}, J=10.3,5.1 \mathrm{~Hz}, 1 \mathrm{H}, \mathrm{C} \underline{\mathrm{H} A r}), 3.30$ (d, $J=9.8 \mathrm{~Hz}, 1 \mathrm{H}, \mathrm{CH}), 3.21$ (d, $J=9.9 \mathrm{~Hz}, 1 \mathrm{H}$, $\mathrm{CH}), 3.09$ (dd, $J=16.0,10.3 \mathrm{~Hz}, 1 \mathrm{H}, \mathrm{C} \underline{\mathrm{H}}), 2.81$ (dd, $J=16.0,5.1 \mathrm{~Hz}, 1 \mathrm{H}, \mathrm{CH} \underline{\mathrm{H}}), 2.34$ (s, $\left.3 \mathrm{H}, \mathrm{CH}_{3}\right), 1.84(\mathrm{~d}, J=8.5 \mathrm{~Hz}, 1 \mathrm{H}, \mathrm{C} \underline{\mathrm{HH}}), 1.63\left(\mathrm{~s}, 3 \mathrm{H}, \mathrm{CH}_{3}\right), 1.59(\mathrm{~d}, J=8.5 \mathrm{~Hz}, 1 \mathrm{H}, \mathrm{CH} \underline{\mathrm{H}})$, 1.55 (s, 3H, $\left.\mathrm{CH}_{3}\right) ;{ }^{13} \mathrm{C}$ NMR (75 MHz, $\mathrm{CDCl}_{3}$ ) $\delta 208.7$ (C), 208.6 (C), 147.3, (C), 146.8 (C), 137.5(C), $135.6(\mathrm{C}), 135.4(\mathrm{C}), 133.1(\mathrm{C}), 129.7(\mathrm{CH}), 129.3(\mathrm{CH}), 129.2(\mathrm{CH}), 128.2(\mathrm{CH})$, $128.1(\mathrm{CH}), 128.0(\mathrm{CH}), 127.03(\mathrm{CH}), 127.01(\mathrm{CH}), 66.1\left(\mathrm{CH}_{2}\right), 60.2(\mathrm{CH}), 59.0(\mathrm{CH}), 58.1$ (C), $58.0(\mathrm{C}), 52.5(\mathrm{CH}), 45.3\left(\mathrm{CH}_{2}\right), 21.2\left(\mathrm{CH}_{3}\right), 19.1\left(\mathrm{CH}_{3}\right), 18.7\left(\mathrm{CH}_{3}\right) ; \mathrm{HRMS}(+\mathrm{p} \mathrm{EI}) \mathrm{m} / z$ calc. for $\mathrm{C}_{32} \mathrm{H}_{30} \mathrm{O}_{2}$ : $446.2240[\mathrm{M}]^{+}$; found: 446.2235; $[\alpha]_{\mathrm{D}}^{22.1}=+30.0\left(c\right.$ 1.0, $\left.\mathrm{CHCl}_{3}\right)$; 97:3 e.r.; HPLC (CHIRALPAK IA, hexane/2-propanol: 95:5, flow rate: $1.0 \mathrm{~mL}$ min-1, detection UV 215 $\mathrm{nm}, 25^{\circ} \mathrm{C}$ ) $\mathrm{t}_{\mathrm{R}}$ of major isomer: $10.580 \mathrm{~min}, \mathrm{t}_{\mathrm{R}}$ of minor isomer: $9.052 \mathrm{~min}$. 


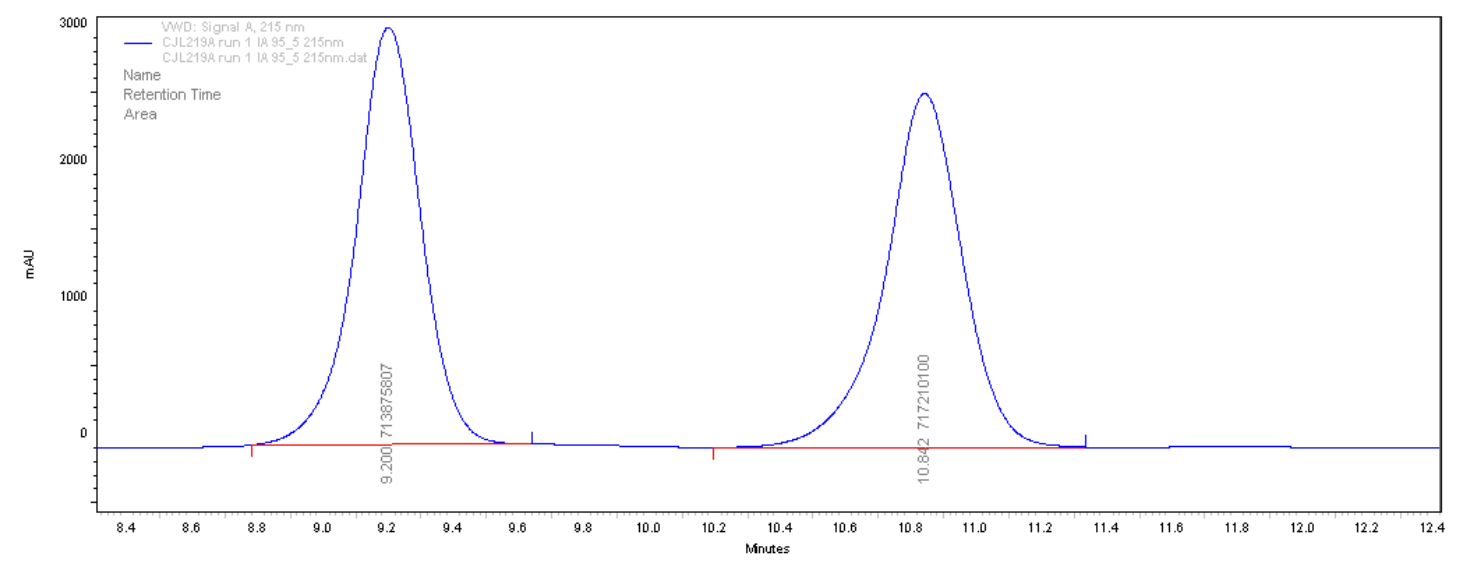

VWD : Signal A,

$215 \mathrm{num}$ Results

\begin{tabular}{rrrrr} 
Retention Time & Area & Area \% & Height & Height \% \\
\hline 9.200 & 713875807 & 49.88 & 51203520 & 54.12 \\
10.842 & 717210100 & 50.12 & 43411752 & 45.88
\end{tabular}

\begin{tabular}{|r|r|r|r|r|}
\hline Totals & 1431085907 & 100.00 & 94615272 & 100.00 \\
\hline
\end{tabular}

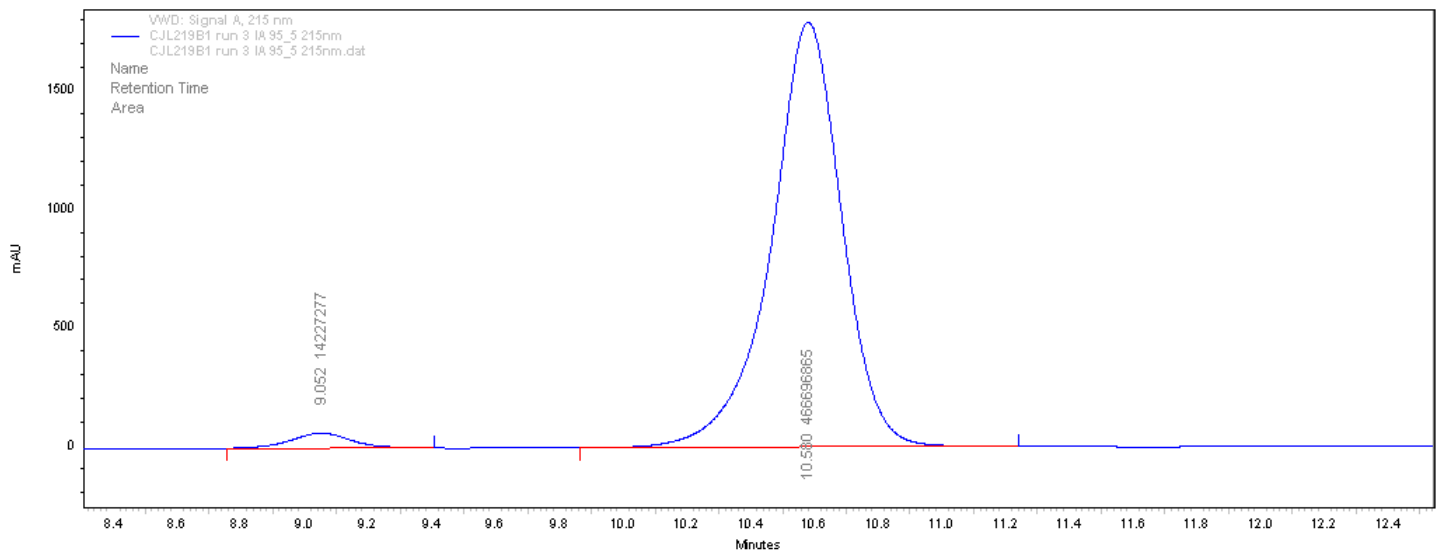

VWD: Signal A,

215 um Results

Retention Time 9.052 $\begin{array}{rr}\text { Area } & \text { Area } \% \\ 14227277 & 2.96 \\ 466696865 & 97.04\end{array}$ $\begin{array}{rr}\text { Height } \\ 2.96 & 1069047\end{array}$ 1069047
30122772 Height \% 10.580 
6-(4-Chloro-3-methoxyphenyl)-1,4-dimethyl-2,3-diphenyl-1,4,4a,6,7,8a-hexahydro-1,4methanonaphthalene-5,8-dione 5p

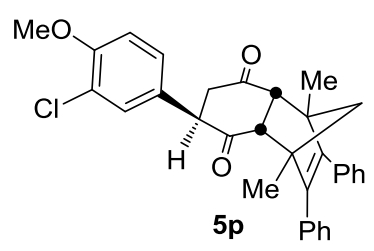

\section{Racemic procedure:}

General racemic procedure was followed. Reacting (4-chloro-3-methoxyphenyl)boronic acid 2p (44.7 mg, 0.24 mmol, 2.4 equiv.), Diels-Alder adduct $4 \mathbf{b}$ (35.4 mg, 0.099 mmol, 1 equiv.), $\mathrm{Pd}(\mathrm{OAc})_{2}$ (1.1 mg, $0.005 \mathrm{mmol}, 0.05$ equiv.) and 1,10-phenanthroline (1.1 mg, $0.006 \mathrm{mmol}$, 0.06 equiv.) in DMF ( $1 \mathrm{~mL}$ ) under at atmosphere of $\mathrm{O}_{2}$ (balloon) at $40{ }^{\circ} \mathrm{C}$ for $24 \mathrm{~h}$.. The resulting crude was purified by silica gel column chromatography (pet. ether/EtOAc 5:1 with $10 \%$ toluene) to yield $\mathbf{5 p}(28.6 \mathrm{mg}, 0.058 \mathrm{mmol}, 58 \%,>20: 1$ d.r. $)$ as a colourless oil.

\section{Enantioselective procedure:}

General enantioselective procedure B was followed with modifications. (4-Chloro-3methoxyphenyl)boronic acid 2 p (44.7 $\mathrm{mg}, 0.24 \mathrm{mmol}, 2.4$ equiv.) was dehydrated under vacuum with a heat gun in the reaction flask to the corresponding boroxine and reacted with Diels-Alder adduct 4b (35.4 mg, 0.099 mmol, 1 equiv.), $\mathrm{Pd}(\mathrm{OAc})_{2}$ (2.2 mg, $0.010 \mathrm{mmol}, 0.10$ equiv.) and ${ }^{t} \mathrm{BuPyOx}$ L-A (2.2 mg, $0.011 \mathrm{mmol}, 0.11$ equiv.) in DMF (1 mL) under at atmosphere of $\mathrm{O}_{2}$ (balloon) at $40{ }^{\circ} \mathrm{C}$ for $24 \mathrm{~h}$. Catalyst and ligand were not premixed. The resulting crude was purified by silica gel column chromatography (5:1 hexane:EtOAc with $10 \%$ toluene) to yield $\mathbf{5 p}$ (25.5 mg, $0.051 \mathrm{mmol}, 51 \%$, >20:1 d.r., 97:3 e.r.) as a colourless oil. $\mathrm{R}_{\mathrm{f}}$ : 0.2 in 5:1 pet. ether:EtOAc; $v_{\max } / \mathrm{cm}^{-1}: 2959,2626,2856,1699,1611,1575,1443,1274$, 1105, 770, 727, 698, ; ${ }^{1} \mathrm{H}$ NMR (300 MHz, $\left.\mathrm{CDCl}_{3}\right) \delta 7.25-7.12(\mathrm{~m}, 6 \mathrm{H}, \mathrm{Ar}-\mathrm{H}), 7.08-7.05$ (m, 1H, Ar-H), $6.98-6.83$ (m, 6H, Ar-H), 3.89 (s, 3H, OMe), 3.85 (dd, $J=11.5,5.0 \mathrm{~Hz}, 1 \mathrm{H}$, CㅂA), $3.32(\mathrm{~d}, J=9.9 \mathrm{~Hz}, 1 \mathrm{H}, \mathrm{CH}), 3.22(\mathrm{~d}, J=9.9 \mathrm{~Hz}, 1 \mathrm{H}, \mathrm{CH}), 3.03(\mathrm{dd}, J=15.9,11.5$ $\mathrm{Hz}, 1 \mathrm{H}, \mathrm{C} \underline{H} \mathrm{H}), 2.78$ (dd, $J=15.9,5.0 \mathrm{~Hz}, 1 \mathrm{H}, \mathrm{CH} \underline{\mathrm{H}}), 1.85$ (d, J = 8.5 Hz, 1H, CㅂH), 1.64 (s, $\left.3 \mathrm{H}, \mathrm{CH}_{3}\right), 1.61(\mathrm{~d}, J=8.6 \mathrm{~Hz}, 1 \mathrm{H}, \mathrm{CH} \underline{\mathrm{H}}), 1.52\left(\mathrm{~s}, 3 \mathrm{H}, \mathrm{CH}_{3}\right) ;{ }^{13} \mathrm{C} \mathrm{NMR}\left(75 \mathrm{MHz}, \mathrm{CDCl}_{3}\right) \delta$ 208.5 (C), 207.7 (C), 154.6 (C), 147.6(C), 146.4 (C), 135.6 (C), 135.2 (C), $130.1(\mathrm{CH}), 129.2$ $(\mathrm{CH}), 129.2(\mathrm{CH}), 129.1(\mathrm{C}), 128.3(\mathrm{CH}), 128.2(\mathrm{CH}), 127.4(\mathrm{CH}), 127.18(\mathrm{CH}) 127.16(\mathrm{CH})$, $123.0(\mathrm{C}), 112.4(\mathrm{CH}), 66.1\left(\mathrm{CH}_{2}\right), 60.4(\mathrm{CH}), 59.2(\mathrm{CH}), 58.4(\mathrm{C}), 58.1(\mathrm{C}), 56.3\left(\mathrm{CH}_{3}\right), 51.4$ $(\mathrm{CH}), 45.6\left(\mathrm{CH}_{2}\right), 19.2\left(\mathrm{CH}_{3}\right), 18.5\left(\mathrm{CH}_{3}\right)$; HRMS (TOF MS ASAP+) $m / z$ calc. for $\mathrm{C}_{32} \mathrm{H}_{29} \mathrm{O}_{3} \mathrm{Cl}$ : $496.1805[\mathrm{M}+\mathrm{H}]^{+}$; found: 496.1801; $[\alpha]_{\mathrm{D}}^{22.1}=+24.0\left(c\right.$ 0.67, $\left.\mathrm{CHCl}_{3}\right) ; 97: 3$ e.r.; HPLC 
(CHIRALPAK IA, hexane/2-propanol: 95:5, flow rate: $1.0 \mathrm{~mL} \mathrm{~min}^{-1}$, detection UV $215 \mathrm{~nm}$, $25{ }^{\circ} \mathrm{C}$ ) $\mathrm{t}_{\mathrm{R}}$ of major isomer: $21.508 \mathrm{~min}, \mathrm{t}_{\mathrm{R}}$ of minor isomer: $20.090 \mathrm{~min}$.

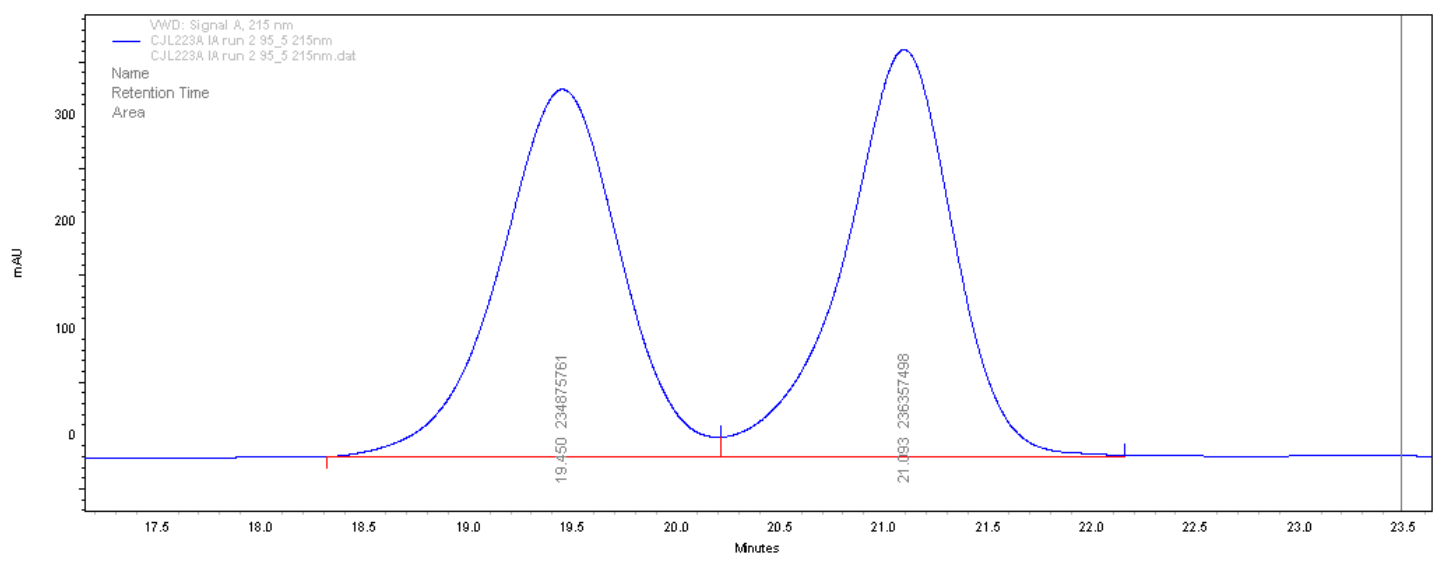

VwD: Signal A,

$215 \mathrm{~nm}$ Results

\begin{tabular}{rrrrrr|}
$\begin{array}{r}\text { Area } \\
\text { Retention Time }\end{array}$ & Area $\%$ & Height & Height \% \\
\hline 19.450 & 234875761 & 49.84 & 5781761 & 47.44 \\
21.093 & 236357498 & 50.16 & 6406758 & 52.56 \\
\hline \multicolumn{2}{|c|}{ Totals } & 471233259 & 100.00 & 12188519 & 100.00 \\
\hline
\end{tabular}

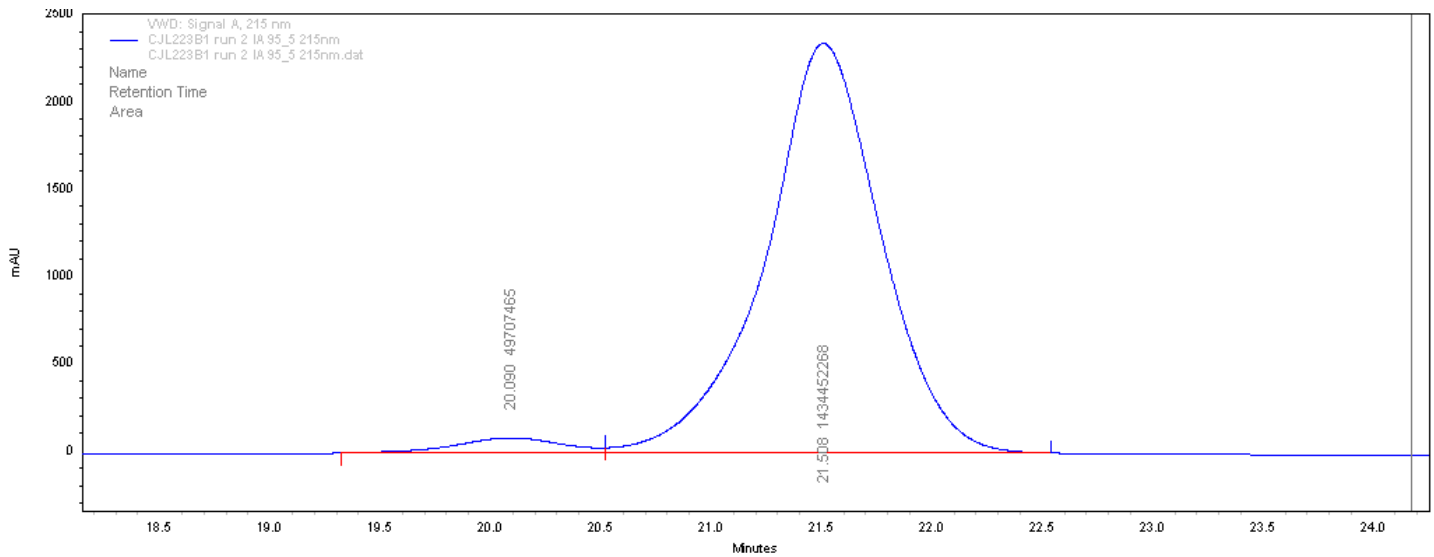

VWD : Signal A,

215 um Results

\begin{tabular}{rrrrr} 
Area & Area \% & Height & Height \% \\
\hline 20.090 & 49707465 & 3.35 & 1461615 & 3.58 \\
21.508 & 1434452268 & 96.65 & 39358109 & 96.42
\end{tabular}

\begin{tabular}{|r|r|r|r|r|}
\hline Totals & 1484159733 & 100.00 & 40819724 & 100.00 \\
\hline
\end{tabular}




\section{6-(4-Fluorophenyl)-1,4-dimethyl-2,3-diphenyl-1,4,4a,6,7,8a-hexahydro-1,4- methanonaphthalene-5,8-dione 5q}

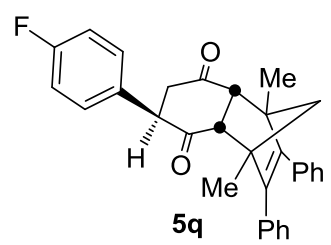

\section{Racemic procedure:}

General racemic procedure was followed. Reacting $p$-fluorobenzene boronic acid $\mathbf{2 q}(33.6 \mathrm{mg}$, 0.24 mmol, 2.4 equiv.) with Diels-Alder adduct $4 \mathbf{b}$ (35.4 mg, $0.099 \mathrm{mmol}, 1.0$ equiv.), $\mathrm{Pd}(\mathrm{OAc})_{2}(1.1 \mathrm{mg}, 0.005 \mathrm{mmol}, 0.05$ equiv.) and 1,10-phenanthroline (1.1 mg, $0.006 \mathrm{mmol}$, 0.06 equiv.) in DMF $(1 \mathrm{~mL})$ under at atmosphere of $\mathrm{O}_{2}$ (balloon) at $40{ }^{\circ} \mathrm{C}$ for $24 \mathrm{~h}$.. The resulting crude was purified by silica gel column chromatography (pet. ether/EtOAc 10:1) to yield 5q (36.0 mg as a mixture of product and starting material $\mathbf{4 b},>20: 1$ d.r.) as a colourless oil.

\section{Enantioselective procedure:}

General enantioselective procedure B was followed. Reacting $p$-fluorobenzene boronic acid $\mathbf{2 q}$ (33.6 mg, 0.24 mmol, 2.4 equiv.), Diels-Alder adduct 4b (35.4 mg, 0.099 mmol, 1.0 equiv.), $\mathrm{Pd}(\mathrm{OAc})_{2}\left(2.2 \mathrm{mg}, 0.010 \mathrm{mmol}, 0.10\right.$ equiv.) and ${ }^{t} \mathrm{BuPyOx} \mathbf{L}-\mathbf{A}(2.1 \mathrm{mg}, 0.011 \mathrm{mmol}, 0.11$ equiv.) in DMF ( $1 \mathrm{~mL})$ under at atmosphere of $\mathrm{O}_{2}$ (balloon) at $40{ }^{\circ} \mathrm{C}$ for $72 \mathrm{~h}$.. The resulting crude was purified by silica gel column chromatography (pet. ether/EtOAc 10:1) and the CSPHPLC analysis carried out at this stage. Further purification was then carried out by recystallisation with DCM/hexanes to removed unknown impurities to yield $\mathbf{5 q}$ (30.2 mg, 0.067 mmol, 67\%, >20:1 d.r., 96:4 e.r.) as an off-white powder.

Mp: $158-162{ }^{\circ} \mathrm{C}$ (hexanes/DCM); $\mathrm{R}_{\mathrm{f}}: 0.2$ in 10:1 pet. ether:EtOAc; $v_{\max } / \mathrm{cm}^{-1}: 2957,2927$, 1699, 1606, 1510, 1488, 1223, 840, 776, $720699 ;{ }^{1} \mathrm{H} \mathrm{NMR}\left(400 \mathrm{MHz}, \mathrm{CDCl}_{3}\right) \delta 7.24-7.18$ (m, 3H, Ar-H), 7.17 - 7.11 (m, 3H, Ar-H), 7.02 (d, J = 11.3, 4H, Ar-H), $6.98-6.91$ (m, 2H, Ar-H), $6.91-6.83$ (m, 2H, Ar-H), 3.92 (dd, $J=11.3,5.0 \mathrm{~Hz}, 1 \mathrm{H}$, C 1H, CH), $3.22(\mathrm{~d}, J=9.8 \mathrm{~Hz}, 1 \mathrm{H}, \mathrm{CH}), 3.05(\mathrm{dd}, J=15.9,11.3 \mathrm{~Hz}, 1 \mathrm{H}, \mathrm{C} \underline{\mathrm{HH}}), 2.79$ (dd, $J=$ $15.9,5.0 \mathrm{~Hz}, 1 \mathrm{H}, \mathrm{CH} \underline{\mathrm{H}}), 1.85(\mathrm{~d}, J=8.5 \mathrm{~Hz}, 1 \mathrm{H}, \mathrm{C} \underline{\mathrm{HH}}), 1.64\left(\mathrm{~s}, 3 \mathrm{H}, \mathrm{CH}_{3}\right), 1.61(\mathrm{~d}, J=8.5 \mathrm{~Hz}$, $1 \mathrm{H}, \mathrm{CH} \underline{\mathrm{H}}), 1.53\left(\mathrm{~s}, 3 \mathrm{H}, \mathrm{CH}_{3}\right) ;{ }^{19} \mathrm{~F}$ NMR $\left(376 \mathrm{MHz}, \mathrm{CDCl}_{3}\right) \delta-114.67$ (m, F-Ar); ${ }^{13} \mathrm{C}$ NMR (75 MHz, $\left.\mathrm{CDCl}_{3}\right) 208.6$ (C), 207.9 (C), 162.3 (C) (d, $\left.J=246.7 \mathrm{~Hz}\right), 147.6$ (C), 146.5 (C), 135.6 (C), $135.3(\mathrm{C}), 131.89$ (C) (d, $J=3.3 \mathrm{~Hz}), 129.85(\mathrm{CH})(\mathrm{d}, J=8.1 \mathrm{~Hz}), 129.2(\mathrm{CH}), 128.3$ $(\mathrm{CH}), 128.2(\mathrm{CH}), 127.2(\mathrm{CH}), 127.1(\mathrm{CH}), 115.91(\mathrm{CH})(\mathrm{d}, J=21.4 \mathrm{~Hz}), 66.1\left(\mathrm{CH}_{2}\right), 60.4$ 
$(\mathrm{CH}), 59.2(\mathrm{CH}), 58.3(\mathrm{C}), 58.1(\mathrm{C}), 51.8(\mathrm{CH}), 45.8\left(\mathrm{CH}_{2}\right), 19.2\left(\mathrm{CH}_{3}\right), 18.6\left(\mathrm{CH}_{3}\right)$, one overlapping CH signal; $\delta$ HRMS (p NSI) $m / z$ calc. for $\mathrm{C}_{31} \mathrm{H}_{27} \mathrm{FO}_{2} \mathrm{NH}_{4}: 468.2333\left[\mathrm{M}+\mathrm{NH}_{4}\right]^{+}$; found: 468.2324; $[\alpha]_{\mathrm{D}}^{20.3}=+12.0\left(c\right.$ 0.67, $\mathrm{CHCl}_{3}$ ); 96:4 e.r.; HPLC (CHIRALPAK IA, hexane/2-propanol: 95:5, flow rate: $1.0 \mathrm{~mL} \mathrm{~min}^{-1}$, detection UV $215 \mathrm{~nm}, 25{ }^{\circ} \mathrm{C}$ ) $\mathrm{t}_{\mathrm{R}}$ of major isomer: $13.067 \mathrm{~min}, \mathrm{t}_{\mathrm{R}}$ of minor isomer: $10.500 \mathrm{~min}$.

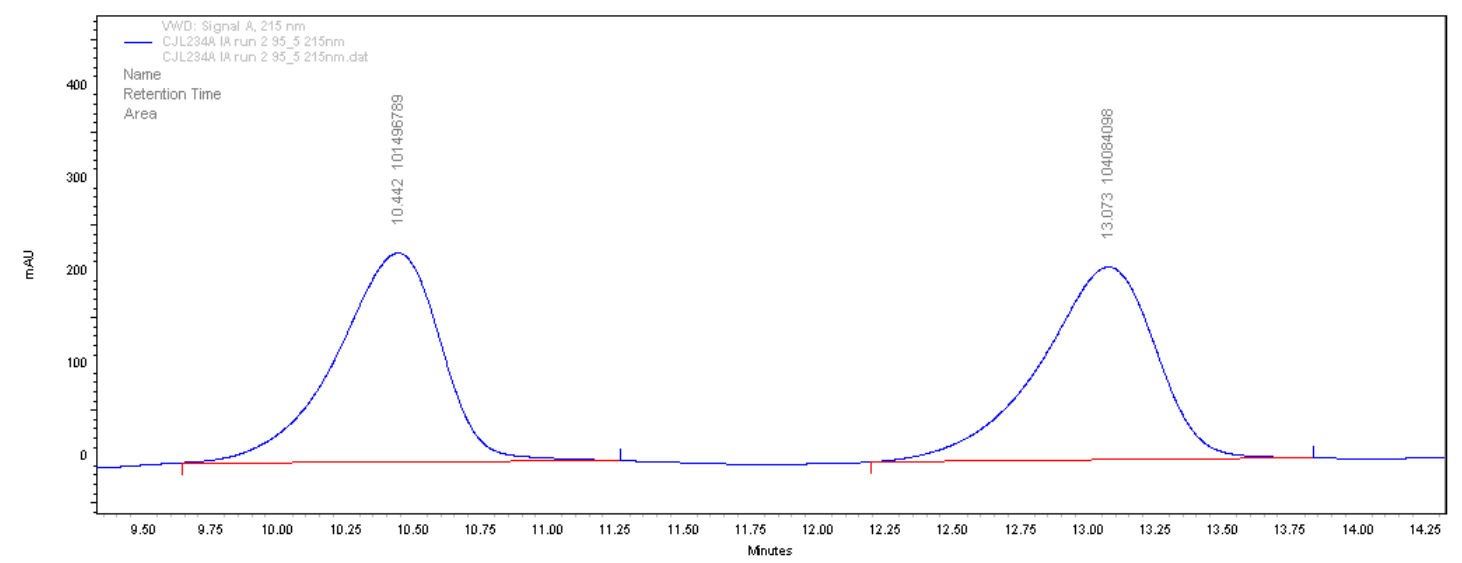

VWD : Signal A,

$215 \mathrm{Jum}$ Results

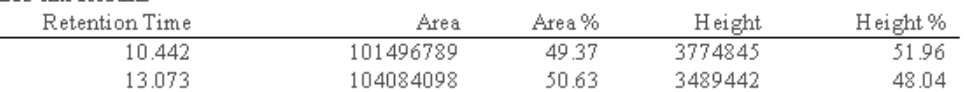

\begin{tabular}{|r|r|r|r|r|}
\hline Totals & 205580887 & 100.00 & 7264287 & 100.00 \\
\hline
\end{tabular}

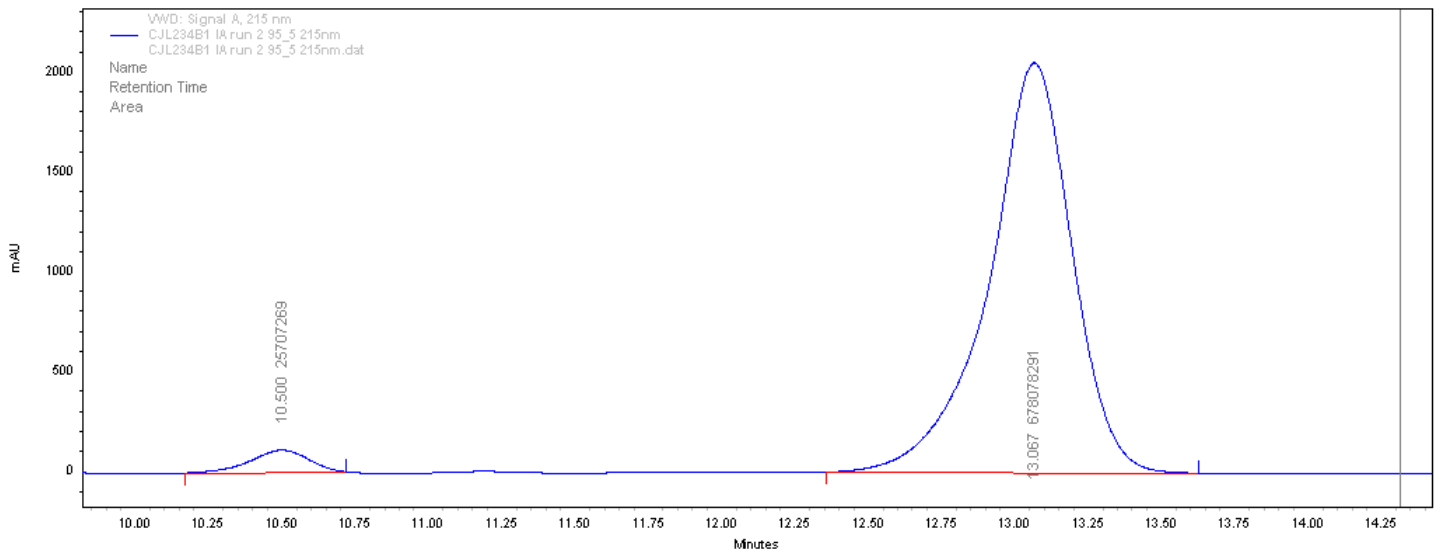

VWD : Signal A,

215 num Results

\begin{tabular}{rrrrr} 
Retention Time & Area & Area\% & Height & Height \% \\
\hline 10.500 & 25707269 & 3.65 & 1862106 & 5.13 \\
13.067 & 678078291 & 96.35 & 34419067 & 94.87
\end{tabular}

\begin{tabular}{|l|r|r|r|r|}
\hline Totals & 703785560 & 100.00 & 36281173 & 100.00 \\
\hline
\end{tabular}




\section{1,4-Dimethyl-5,8-dioxo-2,3-diphenyl-1,4,4a,5,6,7,8,8a-octahydro-1,4- methanonaphthalen-6-yl)phenyl)acetamide $5 r$}

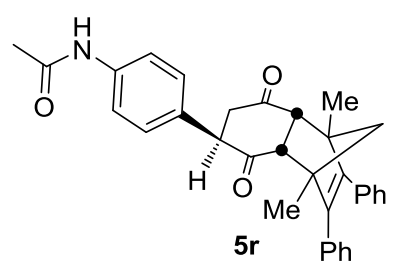

\section{Racemic procedure:}

General racemic procedure was followed with modifications. Reacting $p$ acetamidophenylboronic acid 2r (42.9 mg, 0.24 mmol, 2.4 equiv.), Diels-Alder adduct 4b (35.4 $\mathrm{mg}, 0.099 \mathrm{mmol}, 1.0$ equiv. $), \operatorname{Pd}(\mathrm{OAc})_{2}(1.1 \mathrm{mg}, 0.005 \mathrm{mmol}, 0.05$ equiv.) and 1,10phenanthroline (1.1 mg, $0.006 \mathrm{mmol}, 0.06$ equiv.) in DMF (1 mL) under at atmosphere of $\mathrm{O}_{2}$ (balloon) at $50{ }^{\circ} \mathrm{C}$ for $72 \mathrm{~h}$. The resulting crude was purified by silica gel column chromatography (pet. ether:EtOAc $2: 1 \rightarrow 1: 1)$ to yield $\mathbf{5 r}(37.5 \mathrm{mg}, 0.06 \mathrm{mmol}, 69 \%,>20: 1$ d.r.) as a colourless amorphous solid with $10 \%$ impurities.

\section{Enantioselective procedure:}

General enantioselective procedure $\mathrm{B}$ was followed with modifications. Reacting $p$ acetamidophenylboronic acid $2 \mathbf{r}$ (42.9 mg, $0.24 \mathrm{mmol}, 2.4$ equiv.), Diels-Alder adduct $4 \mathbf{b}$ (35.4 mg, 0.099 mmol, 1.0 equiv.) $\mathrm{Pd}(\mathrm{OAc})_{2}(2.2 \mathrm{mg}, 0.010 \mathrm{mmol}, 0.10$ equiv. $)$ and ${ }^{t} \mathrm{BuPyOx} \mathbf{L}-\mathbf{A}$ ( $2.1 \mathrm{mg}, 0.011 \mathrm{mmol}, 0.11$ equiv.) in DMF (1 mL) under at atmosphere of $\mathrm{O}_{2}$ (balloon) at 40 ${ }^{\circ} \mathrm{C}$ for $96.5 \mathrm{~h}$. The resulting crude was purified by silica gel column chromatography (hexane:EtOAc 2:1 $\rightarrow$ 1:1), then recystallised with DCM/hexanes to removed unknown impurities (after enantiomeric ratio was recorded) to yield $\mathbf{5 r}(20.6 \mathrm{mg}, 0.042 \mathrm{mmol}, 42 \%$, $>$ 20:1 d.r., 98:2 e.r.) as a white powder.

Mp: $114-117{ }^{\circ} \mathrm{C}$ (DCM/hexanes); Rf: 0.2 in 2:1 pet. ether:EtOAc; vmax/ $\mathrm{cm}^{-1}: 3311,1694$, 1600, 1516, 1443, 1411, 745, 726, 698; ${ }^{1} \mathrm{H}$ NMR (300 MHz, $\left.\mathrm{CDCl}_{3}\right) 7.50-7.41$ (m, 3H, ArH), $7.23-7.11$ (m, 6H, Ar-H), 7.05 - 6.97 (m, 2H, Ar-H), 6.97 - 6.84 (m, 3H, Ar-H), 3.90 (dd, $J=10.4,5.1 \mathrm{~Hz}, 1 \mathrm{H}, \mathrm{C} \underline{\mathrm{H} A r}), 3.29$ (d, $J=9.9 \mathrm{~Hz}, 1 \mathrm{H}, \mathrm{CH}), 3.20$ (d, $J=9.9 \mathrm{~Hz}, 1 \mathrm{H}, \mathrm{CH}), 3.06$ (dd, $J=16.0,10.4 \mathrm{~Hz}, 1 \mathrm{H}, \mathrm{C} \underline{\mathrm{H}} \mathrm{H}$ ), 2.79 (dd, $J=16.0,5.1 \mathrm{~Hz}, 1 \mathrm{H}, \mathrm{CH} \underline{\mathrm{H}}), 2.14$ (s, 3H, $\left.\mathrm{NCOCH}_{3}\right), 1.84$ (d, J=8.6 Hz, 1H, $\left.\underline{\mathrm{HH}}\right), 1.62\left(\mathrm{~s}, 3 \mathrm{H}, \mathrm{CH}_{3}\right), 1.59$ (d, $\left.J=8.6 \mathrm{~Hz}, 1 \mathrm{H}, \mathrm{CH} \underline{\mathrm{H}}\right)$, 1.54 (s, 3H, $\left.\mathrm{CH}_{3}\right) ;{ }^{13} \mathrm{C}$ NMR (101 MHz, $\left.\mathrm{CDCl}_{3}\right) 208.7$ (C), 208.5 (C), 168.5 (C), 147.3 (C), $146.7(\mathrm{C}), 137.5(\mathrm{C}), 135.6(\mathrm{C}), 135.3(\mathrm{C}), 131.8(\mathrm{C}), 129.3(\mathrm{CH}), 129.2(\mathrm{CH}), 128.7(\mathrm{CH})$, $128.3(\mathrm{CH}), 128.2(\mathrm{CH}), 127.1(\mathrm{CH}), 120.3(\mathrm{CH}), 66.1\left(\mathrm{CH}_{2}\right), 60.2(\mathrm{CH}), 59.1(\mathrm{CH}), 58.1(\mathrm{C})$, 
$58.1(\mathrm{C}), 52.2(\mathrm{CH}), 45.3\left(\mathrm{CH}_{2}\right), 24.7\left(\mathrm{CH}_{3}\right), 19.1\left(\mathrm{CH}_{3}\right), 18.7\left(\mathrm{CH}_{2}\right)$, and $1 \times$ overlapping $\mathrm{CH}$ signal; $\delta$ HRMS (FTMS + p NSI) $m / z$ calc. for $\mathrm{C}_{33} \mathrm{H}_{32} \mathrm{O}_{3} \mathrm{NH}_{4}: 507.2642\left[\mathrm{M}+\mathrm{NH}_{4}{ }^{+}\right]+$; found: 507.2637; $[\alpha]_{\mathrm{D}}^{21.5}=+27.3\left(\mathrm{c}\right.$ 0.37, $\left.\mathrm{CHCl}_{3}\right)$; 98:2 e.r.; HPLC (CHIRALPAK IB, hexane/2propanol: $85: 15$, flow rate: $0.75 \mathrm{~mL} \mathrm{~min}^{-1}$, detection $\mathrm{UV} 215 \mathrm{~nm}, 25^{\circ} \mathrm{C}$ ) $\mathrm{t}_{\mathrm{R}}$ of major isomer: $27.835 \mathrm{~min}, \mathrm{t}_{\mathrm{R}}$ of minor isomer: $37.987 \mathrm{~min}$.

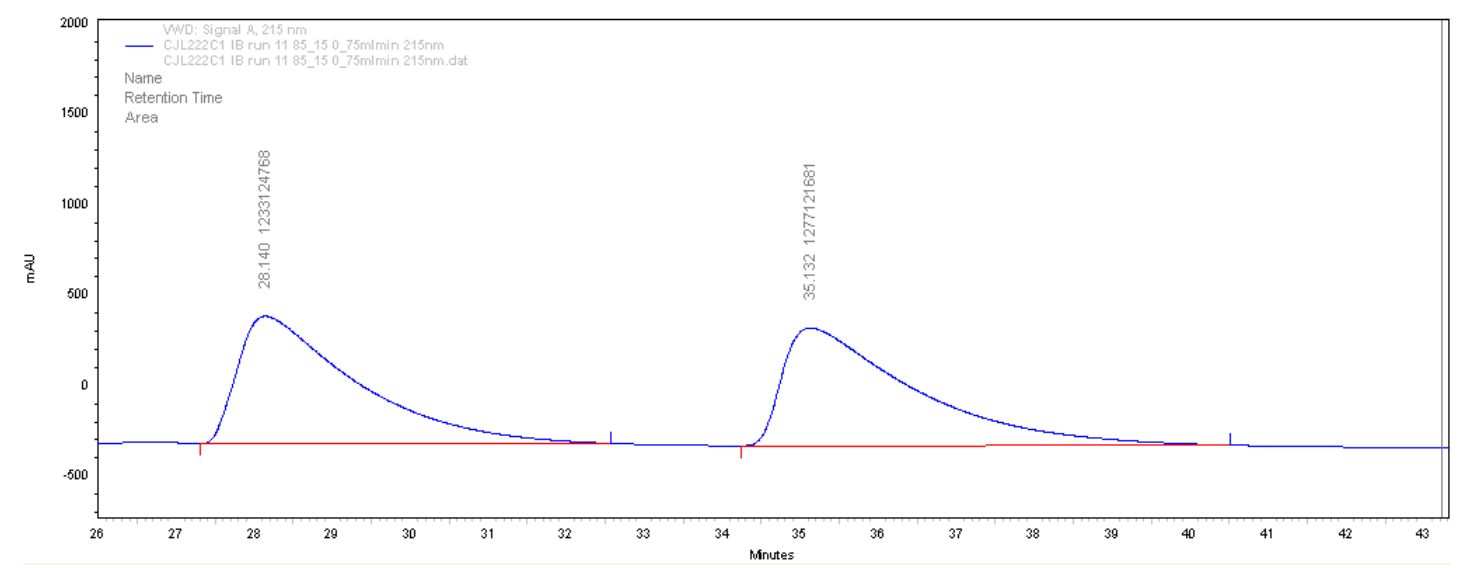

VwD: Signal A,

$215 \mathrm{~nm}$ Results

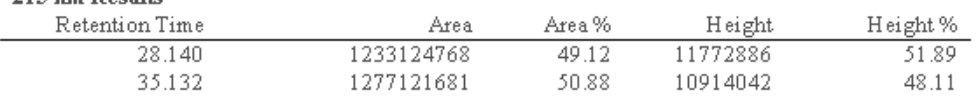

\begin{tabular}{|r|r|r|r|r|}
\hline Totals & 2510246449 & 100.00 & 22686928 & 100.00 \\
\hline
\end{tabular}

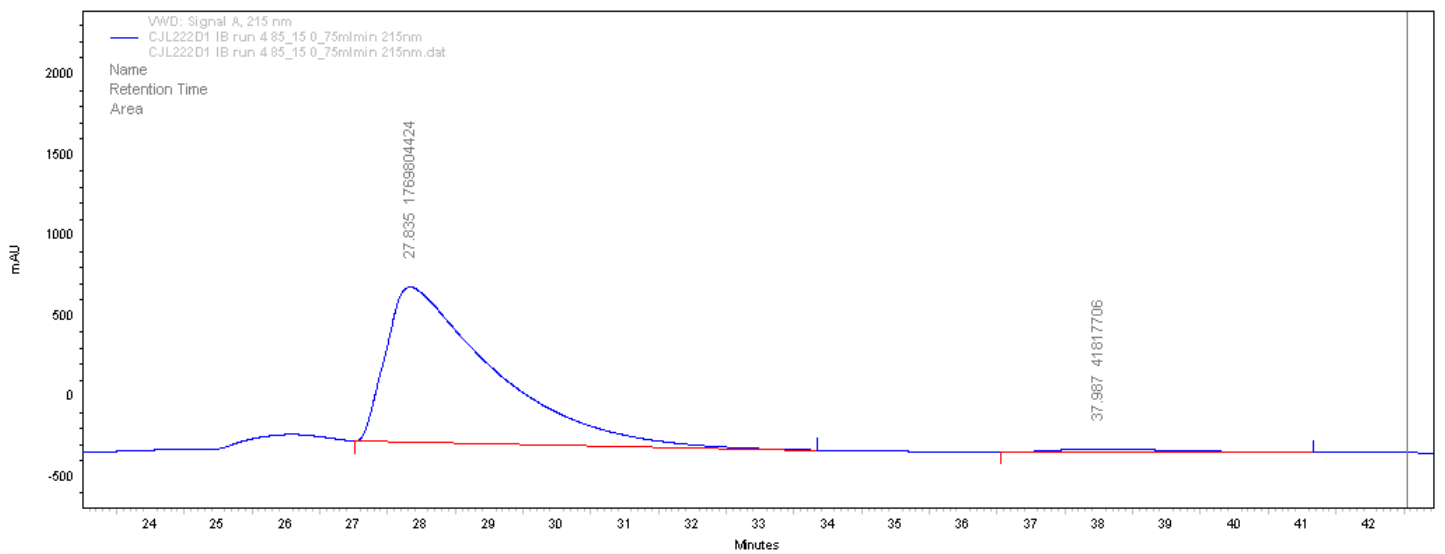

VWD: Signal A,

215 um Results

Retention Time

\begin{tabular}{rrrrr} 
Area & Area $\%$ & Height & Height \% \\
\hline 27.835 & 1769804424 & 97.69 & 16156114 & 97.96 \\
37.987 & 41817706 & 2.31 & 335909 & 2.04
\end{tabular}

Totals

\begin{tabular}{l|l|l}
1811622130 & 100.00 & 16492023 \\
\hline
\end{tabular} 


\section{Ethyl 4-(1,4-dimethyl-5,8-dioxo-2,3-diphenyl-1,4,4a,5,6,7,8,8a-octahydro-1,4- methanonaphthalen-6-yl)benzoate $5 \mathrm{~s}$}

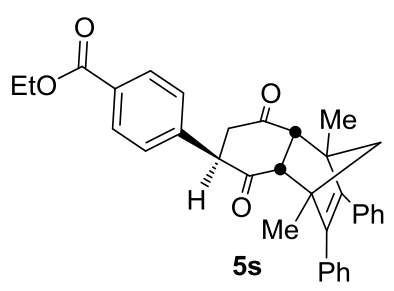

\section{Racemic procedure:}

General racemic procedure was followed with modifications. Reacting $p$ ethoxycarbonylphenylboronic acid $2 \mathrm{~s}$ (46.6 mg, $0.24 \mathrm{mmol}, 2.4$ equiv.), Diels-Alder adduct $4 \mathbf{b}$ (35.4 mg, 0.099 mmol, 1.0 equiv.), $\mathrm{Pd}(\mathrm{OAc})_{2}$ (1.1 mg, $0.005 \mathrm{mmol}, 0.05$ equiv.) and 1,10phenanthroline (1.1 mg, $0.006 \mathrm{mmol}, 0.06$ equiv.) in DMF (1 mL) under at atmosphere of $\mathrm{O}_{2}$ (balloon) at $50{ }^{\circ} \mathrm{C}$ for $72 \mathrm{~h}$. The resulting crude was purified by silica gel column chromatography (pet. ether:EtOAc 5:1 + 10\% toluene). Phenol was removed by sat. $\mathrm{K}_{2} \mathrm{CO}_{3}$ wash $(3 \times 10 \mathrm{~mL})$ to yield $\mathbf{5 s}(22.8 \mathrm{mg}, 0.06 \mathrm{mmol}, 45 \%,>20: 1 \mathrm{~d} . r$. $)$ as a colourless amorphous solid.

\section{Enantioselective procedure:}

General enantioselective procedure $\mathrm{B}$ was followed with modifications. Reacting $p$ ethoxycarbonylphenylboronic acid $2 \mathrm{~s}$ (42.9 mg, $0.24 \mathrm{mmol}, 2.4$ equiv.), Diels-Alder adduct $4 \mathbf{b}$ (35.4 mg, 0.099 mmol, 1.0 equiv.), $\mathrm{Pd}(\mathrm{OAc})_{2}\left(1.1 \mathrm{mg}, 0.005 \mathrm{mmol}, 0.005\right.$ equiv.) and ${ }^{\mathrm{t}} \mathrm{BuPyOx}$ L-A (1.2 mg, 0.006 mmol, 0.06 equiv.) in $\mathrm{DMF}(1 \mathrm{~mL})$ under at atmosphere of $\mathrm{O}_{2}$ (balloon) at $50{ }^{\circ} \mathrm{C}$ for $24 \mathrm{~h}$. An additional portion of $\mathrm{Pd}(\mathrm{OAc})_{2}\left(1.1 \mathrm{mg}, 0.005 \mathrm{mmol}, 0.05\right.$ equiv.), ${ }^{t} \mathrm{BuPyOx}$ L-A (1.2 mg, $0.006 \mathrm{mmol}, 0.06$ equiv.) p-ethoxycarbonylphenylboronic acid $2 \mathrm{~s}$ (37.8 mg, 0.20 mmol, 2.0 equiv.) and the reaction was stirred at $50{ }^{\circ} \mathrm{C}$ under an atmosphere of $\mathrm{O}_{2}$ (balloon) for $48 \mathrm{~h}$ (72 h total). The reaction was diluted with 2:1 Et $2 \mathrm{O}:$ EtOAc $(15 \mathrm{~mL})$ and washed with sat. $\mathrm{K}_{2} \mathrm{CO}_{3}(3 \times 10 \mathrm{~mL})$, water $(2 \times 10 \mathrm{~mL})$, brine $(10 \mathrm{~mL})$ and dried over $\mathrm{MgSO}_{4}$. Solvent was removed under reduced pressure. The resulting crude was purified by silica gel column chromatography (hexane:EtOAc $10: 1 \rightarrow 5: 1)$ to yield $\mathbf{5 s}(6.6 \mathrm{mg}, 0.013 \mathrm{mmol}, 13 \%,>20: 1$ d.r., 95:5 e.r.) as an off-white amorphous solid.

Rf: 0.2 in 5:1 pet. ether:EtOAc; $v \max / \mathrm{cm}^{-1}: 2958,2927,2870,1701,1699,1524,1488,1443$, 1275, 1181, 1105, 1021, 800, 728, 698; ${ }^{1} \mathrm{H}$ NMR (300 MHz, $\left.\mathrm{CDCl}_{3}\right) \delta 7.99-7.89$ (m, 2H, ArH), $7.17-6.99$ (m, 8H, Ar-H), $6.92-6.75$ (m, 4H, Ar-H), 4.30 (q, J = 7.1 Hz, 2H, $\left.\underline{\mathrm{H}}_{2} \mathrm{CH}_{3}\right)$, 3.92 (dd, $J=11.6,5.1 \mathrm{~Hz}, 1 \mathrm{H}, \mathrm{C} \underline{\mathrm{H} A r}), 3.25(\mathrm{~d}, J=9.9 \mathrm{~Hz}, 1 \mathrm{H}, \mathrm{CH}), 3.16$ (d, $J=9.9 \mathrm{~Hz}, 1 \mathrm{H}$, 
CH), 3.03 (dd, $J=15.9,11.6 \mathrm{~Hz}, 1 \mathrm{H}, \mathrm{C} \underline{\mathrm{H}} \mathrm{H}), 2.73(\mathrm{dd}, J=15.9,5.1 \mathrm{~Hz}, 1 \mathrm{H}, \mathrm{CH} \underline{\mathrm{H}}), 1.78$ (d, $J$ $=8.6 \mathrm{~Hz}, 1 \mathrm{H}, \mathrm{C} \underline{\mathrm{HH}}), 1.57\left(\mathrm{~s}, 3 \mathrm{H}, \mathrm{CH}_{3}\right), 1.53(\mathrm{~d}, J=8.6 \mathrm{~Hz}, 1 \mathrm{H}, \mathrm{CH} \underline{\mathrm{H}}), 1.45\left(\mathrm{~s}, 3 \mathrm{H}, \mathrm{CH}_{3}\right), 1.31$ (t, $\left.J=7.1 \mathrm{~Hz}, 3 \mathrm{H}, \mathrm{CH}_{2} \underline{\mathrm{CH}}_{3}\right) ;{ }^{13} \mathrm{C} \mathrm{NMR}\left(75 \mathrm{MHz}, \mathrm{CDCl}_{3}\right) \delta 208.1$ (C), 207.5 (C), 166.3 (C), 147.7 (C), 146.4 (C), 141.1 (C), 135.6 (C), 135.2 (C), 130.2 (CH), 130.0 (C), 129.25 (CH), 129.23(CH), $128.35(\mathrm{CH}), 128.32(\mathrm{CH}), 128.2(\mathrm{CH}), 127.2(\mathrm{CH}), 66.1\left(\mathrm{CH}_{2}\right), 61.2\left(\mathrm{CH}_{2}\right), 60.4$ $(\mathrm{CH}), 59.4(\mathrm{CH}), 58.4(\mathrm{C}), 58.1(\mathrm{C}), 52.5(\mathrm{CH}), 45.5\left(\mathrm{CH}_{2}\right), 19.2\left(\mathrm{CH}_{3}\right), 18.5\left(\mathrm{CH}_{3}\right), 14.5$ $\left(\mathrm{CH}_{3}\right), 1 \times$ overlapping $\mathrm{CH}$ signal; HRMS (TOF MS ASAP +) $m / z$ calc. for $\mathrm{C}_{34} \mathrm{H}_{31} \mathrm{O}_{4}: 203.2222$ [M-H $]^{+}$; found: 503.2223; $[\alpha]_{\mathrm{D}}^{22.3}=+21.7\left(\mathrm{c} 0.5, \mathrm{CHCl}_{3}\right)$; 95:5 e.r.; HPLC (CHIRALPAK IA, hexane/2-propanol: 95:5, flow rate: $1.00 \mathrm{~mL} \mathrm{~min}{ }^{-1}$, detection $\mathrm{UV} 215 \mathrm{~nm}, 25{ }^{\circ} \mathrm{C}$ ) $\mathrm{t}_{\mathrm{R}}$ of major isomer: $25.535 \mathrm{~min}, \mathrm{t}_{\mathrm{R}}$ of minor isomer: $22.280 \mathrm{~min}$.
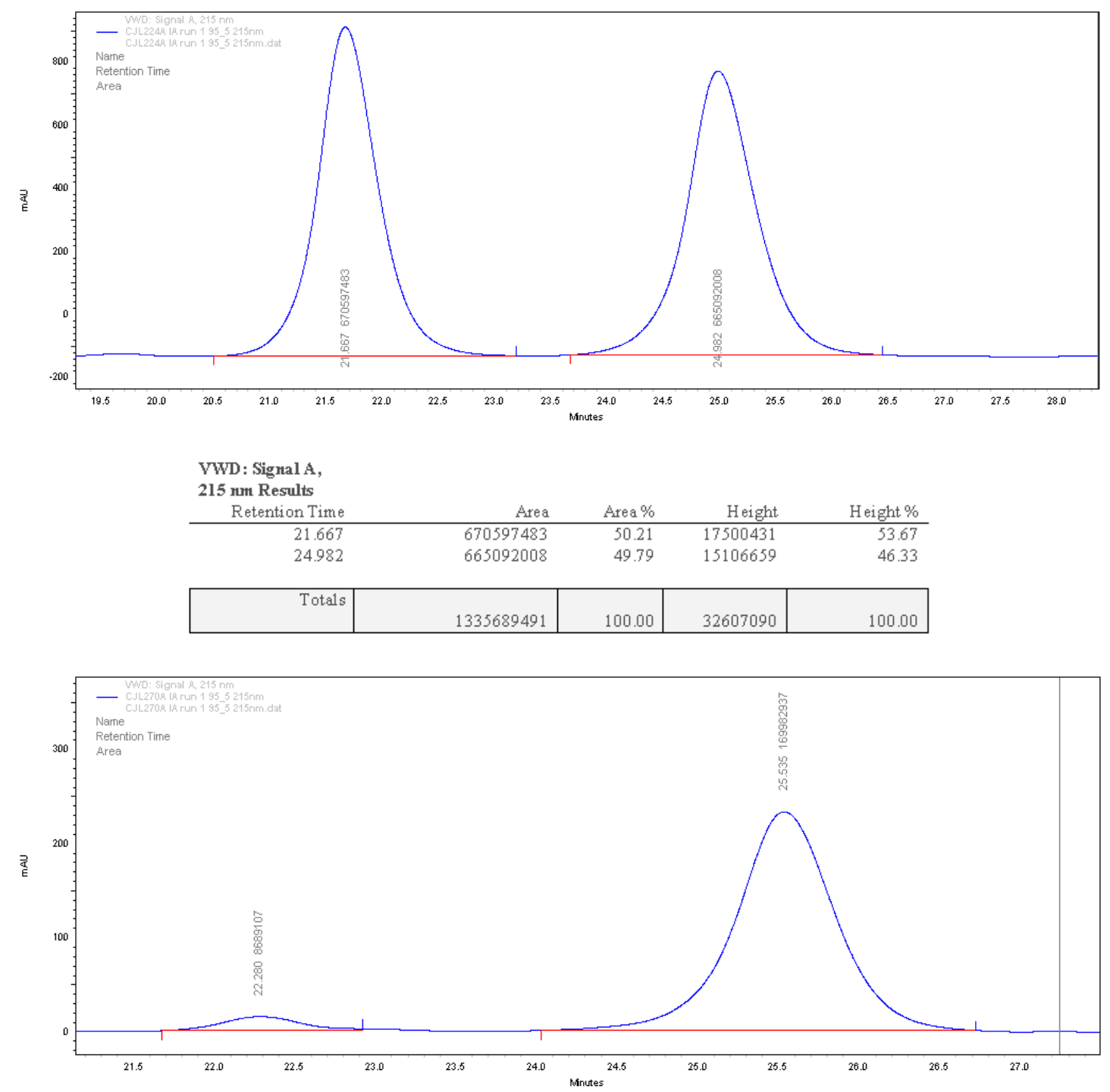
VWD : Signal A

$215 \mathrm{~nm}$ Results

Retention Time

22.280

Area

Area \%

Height

Height \%

25.535

169982937

95.14

3898254

93.98

Totals

178672044

4148103

100.00 
Quinone Products

14-(4-Methoxyphenyl)-9,10-dihydro-9,10-[1,2]benzenoanthracene-13,16-dione 7

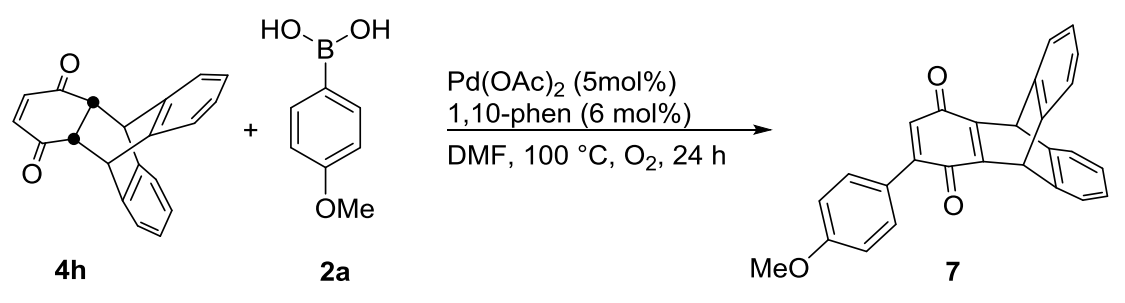

Diels-Alder adduct $\mathbf{4 h}(28.4 \mathrm{mg}, 0.10 \mathrm{mmol}, 1.0$ equiv.) and $p$-methoxyphenylboronic acid $\mathbf{2 a}$ (38.4 mg, $2.5 \mathrm{mmol}, 2.5$ equiv.) were added to the reaction flask along with $\mathrm{Pd}(\mathrm{OAc}) 2(1.1 \mathrm{mg}$, 0.005 mmol, 0.05 equiv.), 1,10-phenanthroline ( $0.9 \mathrm{mg}, 0.05 \mathrm{mmol}, 0.05$ equiv.), and DMF (1 $\mathrm{mL}$ ). The reaction was stirred at $100{ }^{\circ} \mathrm{C}$, under an atmosphere of $\mathrm{O}_{2}$ (balloon) for $24 \mathrm{~h}$. The mixture was diluted with 2:1 $\mathrm{Et}_{2} \mathrm{O}$ :EtOAc $(15 \mathrm{~mL})$ and washed with $\mathrm{H}_{2} \mathrm{O}(3 \times 10 \mathrm{~mL})$ and brine $(1 \times 10 \mathrm{~mL})$. The combined organic layers were dried over $\mathrm{MgSO}_{4}$ and solvent was removed under reduced pressure. The resulting crude was purified by silica gel column chromatography (10:1 Hex:EtOAc) to yield 7 (16.7 $\mathrm{mg}, 0.043 \mathrm{mmol}, 43 \%)$ as a bright orange oil.

$\mathrm{R}_{\mathrm{f}}$ : 0.4 in 5:1 pet. ether:EtOAc; $v_{\max } / \mathrm{cm}^{-1}: 2930,2835,1655,1641,1619,1598,1580,1509$, 1456, 1267, 1254, 1176, 1030, 832, 752; ${ }^{1} \mathrm{H}$ NMR (300 MHz, $\left.\mathrm{CDCl}_{3}\right) \delta 7.49-7.42$ (m, 4H, Ar-H), 7.39 (d, $J=8.9$ Hz, 2H, Ar-H), $7.08-7.01$ (m, 4H, Ar-H), 6.93 (d, J=8.9 Hz, 2H, Ar$\mathrm{H}), 6.63(\mathrm{~s}, 1 \mathrm{H},=\mathrm{CH}), 5.88(\mathrm{~s}, 1 \mathrm{H}, \mathrm{CH}), 5.84(\mathrm{~s}, 1 \mathrm{H}, \mathrm{CH}), 3.84\left(\mathrm{~s}, 3 \mathrm{H}, \mathrm{OCH}_{3}\right) ;{ }^{13} \mathrm{C} \mathrm{NMR}(75$ $\left.\mathrm{MHz}, \mathrm{CDCl}_{3}\right) \delta 183.8(\mathrm{C}), 183.5(\mathrm{C}), 161.4(\mathrm{C}), 152.2(\mathrm{C}), 152.0(\mathrm{C}), 144.8(\mathrm{C}), 144.1(\mathrm{C})$, $144.0(\mathrm{C}), 131.0(\mathrm{CH}), 130.3(\mathrm{CH}), 125.73(\mathrm{CH}), 125.69(\mathrm{CH}), 125.5(\mathrm{C}), 124.6(\mathrm{CH}), 124.5$ $(\mathrm{CH}), 114.2(\mathrm{CH}), 55.5\left(\mathrm{CH}_{3}\right), 48.0(\mathrm{CH}), 47.5(\mathrm{CH})$; HRMS (FTMS + p NSI) $\mathrm{m} / \mathrm{z}$ calc. for $\mathrm{C}_{27} \mathrm{H}_{19} \mathrm{O}_{3}$ : $391.1329 .1172[\mathrm{M}+\mathrm{H}]^{+}$; found: 391.1331. 


\section{6-(4-Methoxyphenyl)-1,2,3,4-tetrahydro-1,4-methanonaphthalene-5,8-dione 9}

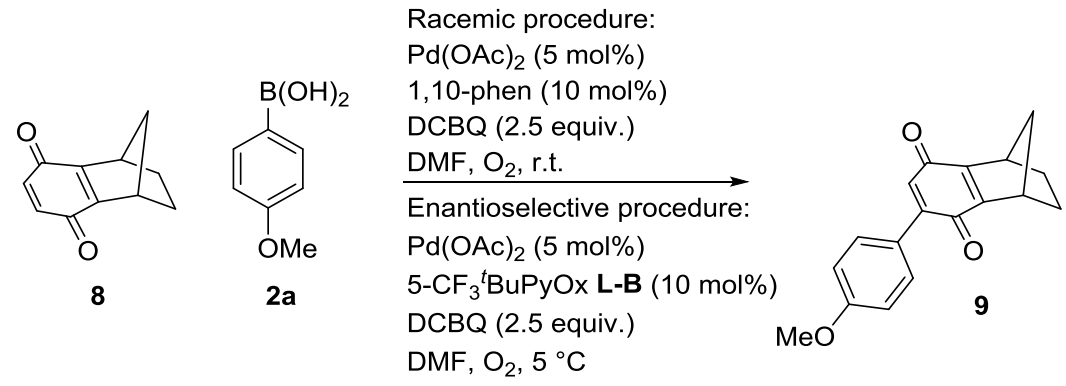

\section{Racemic procedure:}

Quinone 8 (17.7 mg, $0.10 \mathrm{mmol}, 1.0$ equiv.) and $p$-methoxyphenylboronic acid 2a (37.3 mg, $2.5 \mathrm{mmol}, 2.5$ equiv. $)$ were added to the reaction flask along with $\mathrm{Pd}(\mathrm{OAc})_{2}(1.1 \mathrm{mg}, 0.005$ mmol, 0.05 equiv.), 1,10-phenanthroline (1.8 mg, $0.01 \mathrm{mmol}, 0.1$ equiv.), DCBQ (44.2 mg, 0.25 mmol, 2.5 equiv.) and DMF (1 mL). The resulting mixture was stirred at room temperature, under an atmosphere of $\mathrm{O}_{2}$ (balloon) for $16 \mathrm{~h}$. The reaction mixture was diluted with 2:1 $\mathrm{Et}_{2} \mathrm{O}:$ EtOAc $(15 \mathrm{~mL})$ and washed with $\mathrm{H}_{2} \mathrm{O}(3 \times 10 \mathrm{~mL})$ and brine $(1 \times 10 \mathrm{~mL})$. The combined organic layers were dried over $\mathrm{MgSO}_{4}$ and solvent was removed under reduced pressure. The resulting crude product was purified by silica gel column chromatography (10:1 pet. ether:EtOAc) to yield $9(24.5 \mathrm{mg}, 0.084 \mathrm{mmol}, 84 \%)$ as a bright orange oil.

\section{Enantioselective procedure:}

Quinone 8 (17.4 mg, 0.099 mmol, 1.0 equiv.) and $p$-methoxyphenylboronic acid 2a (37.5 mg, $2.5 \mathrm{mmol}, 2.5$ equiv.) were added to the reaction flask along with $\mathrm{Pd}(\mathrm{OAc})_{2}(1.1 \mathrm{mg}, 0.005$ mmol, 0.05 equiv.), 5-CF ${ }^{t}$ BuPyOx L-B (2.7 mg, $0.01 \mathrm{mmol}, 0.1$ equiv.), DCBQ (44.5 mg, $0.25 \mathrm{mmol}, 2.5$ equiv. $)$ and DMF (1 mL). The reaction was stirred at room temperature, under an atmosphere of $\mathrm{O}_{2}$ (balloon) for $25 \mathrm{~h}$. The reaction mixture was diluted with 2:1 $\mathrm{Et}_{2} \mathrm{O}:$ EtOAc $(15 \mathrm{~mL})$ and washed with $\mathrm{H}_{2} \mathrm{O}(3 \times 10 \mathrm{~mL})$ and brine $(1 \times 10 \mathrm{~mL})$. The combined organic layers were dried over $\mathrm{MgSO}_{4}$ and solvent was removed under reduced pressure. The resulting crude was purified by silica gel column chromatography (10:1 hexanes:EtOAc) to yield 9 (27.0 mg, $0.096 \mathrm{mmol}, 96 \%, 63: 37$ e.r.) as a bright orange oil.

$\mathrm{R}_{\mathrm{f}}$ : 0.28 in 10:1 pet. ether:EtOAc; $v_{\max } / \mathrm{cm}^{-1}: 2965,2867,2841,1644,1599,1579,1562,1449$, 1089, 1030, 1015, 835, 798, 724; ${ }^{1} \mathrm{H}$ NMR $\left(300 \mathrm{MHz}, \mathrm{CDCl}_{3}\right) \delta 7.43$ (d, J=8.9 Hz, 2H, Ar$\mathrm{H}), 6.94(\mathrm{~d}, J=8.9 \mathrm{~Hz}, 2 \mathrm{H}, \mathrm{Ar}-\mathrm{H}), 6.61(\mathrm{~s}, 1 \mathrm{H},=\mathrm{CH}), 3.84\left(\mathrm{~s}, 3 \mathrm{H}, \mathrm{OCH}_{3}\right), 3.57-3.53(\mathrm{~m}, 1 \mathrm{H}$, $\mathrm{CH}), 3.53-3.50(\mathrm{~m}, 1 \mathrm{H}, \mathrm{CH}), 2.00-1.89\left(\mathrm{~m}, 2 \mathrm{H}, \mathrm{CH}_{2}\right), 1.73-1.64(\mathrm{~m}, 1 \mathrm{H}, \mathrm{C} \underline{\mathrm{HH}}), 1.42(\mathrm{dt}$, $J=9.1,1.4 \mathrm{~Hz}, 1 \mathrm{H}, \mathrm{CH} \underline{\mathrm{H}}), 1.27-1.18\left(\mathrm{~m}, 2 \mathrm{H}, \mathrm{CH}_{2}\right) ;{ }^{13} \mathrm{C} \mathrm{NMR}\left(75 \mathrm{MHz}, \mathrm{CDCl}_{3}\right) \delta 184.8(\mathrm{C})$, $184.3(\mathrm{C}), 161.2(\mathrm{C}), 151.9(\mathrm{C}), 151.6(\mathrm{C}), 145.4(\mathrm{C}), 131.2(\mathrm{CH}), 131.0(\mathrm{CH}), 125.8(\mathrm{C}), 114.1$ 
$(\mathrm{CH}), 55.5\left(\mathrm{CH}_{3}\right), 47.9\left(\mathrm{CH}_{2}\right), 41.2(\mathrm{CH}), 40.8(\mathrm{CH}), 25.32\left(\mathrm{CH}_{2}\right), 25.27\left(\mathrm{CH}_{2}\right)$; HRMS (FTMS + p NSI) $m / z$ calc. for $\mathrm{C}_{18} \mathrm{H}_{17} \mathrm{O}_{3}: 281.1172[\mathrm{M}+\mathrm{H}]^{+}$; found: $281.1175 ;[\alpha]_{\mathrm{D}}^{21.0}=-32.0(c$ 1.0, $\mathrm{CHCl}_{3}$ ); 63:37 e.r.; HPLC (CHIRALPAK IA, hexane/2-propanol: 95:5, flow rate: $1.0 \mathrm{~mL} \mathrm{~min}{ }^{-}$ ${ }^{1}$, detection UV $254 \mathrm{~nm}, 25^{\circ} \mathrm{C}$ ) $\mathrm{t}_{\mathrm{R}}$ of major isomer: $13.832 \mathrm{~min}, \mathrm{t}_{\mathrm{R}}$ of minor isomer: 15.015 $\min$.
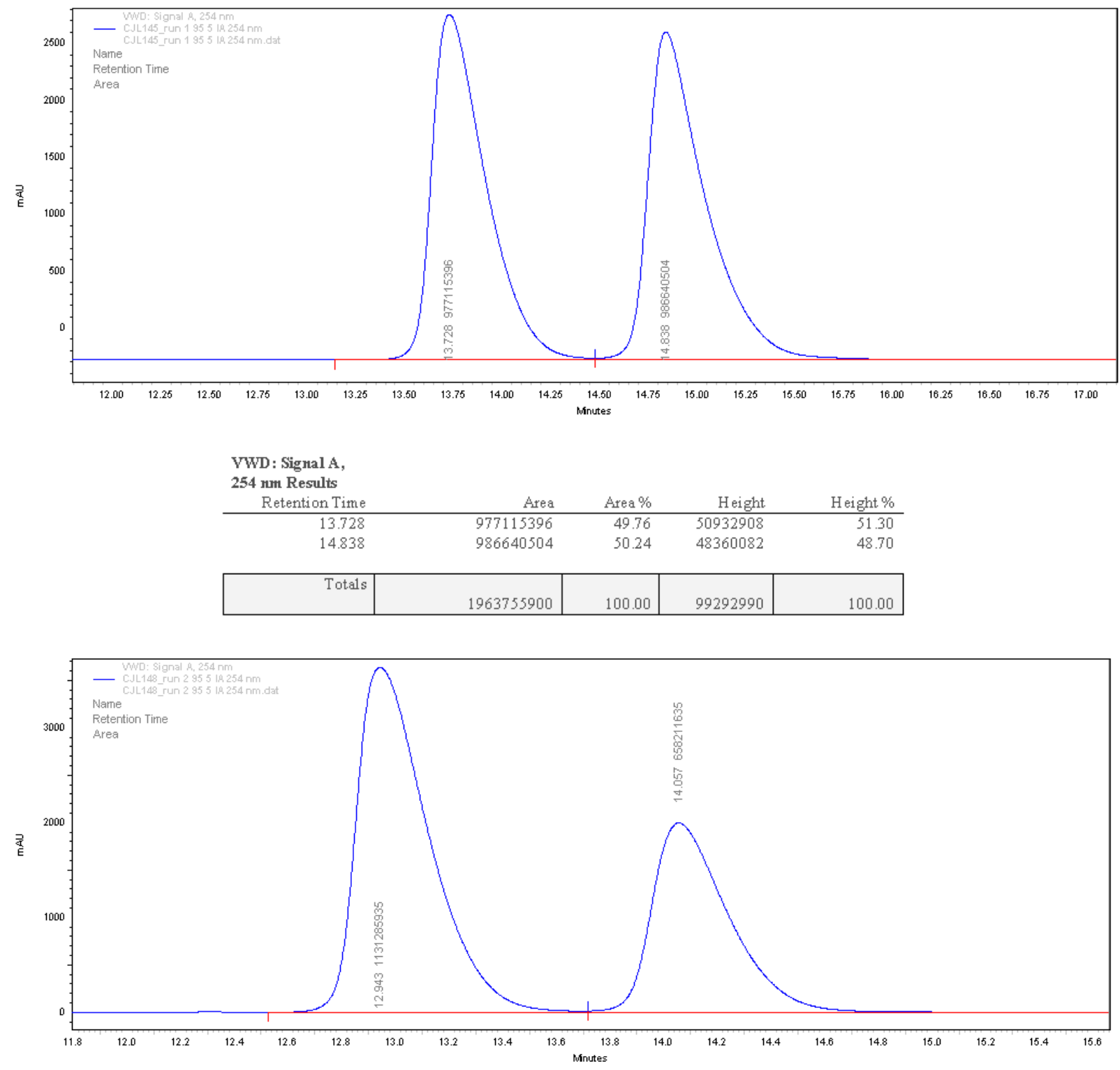

VWD : Signal A,

254 Ium Results

\begin{tabular}{rrrrrr} 
Retention Time & Area & Area $\%$ & Height & Height \% \\
\hline 12.943 & 1131285935 & 63.22 & 60949290 & 64.55 \\
14.057 & 658211635 & 36.78 & 33475151 & 35.45 \\
\hline \multicolumn{2}{|c|}{ Totals } & 1789497570 & 100.00 & 94424441 & 100.00 \\
\hline
\end{tabular}




\section{X-Ray Crystallography Data}

The absolute stereochemistry of the major enantiomer of $\mathbf{5 a}$ was determined by single crystal $\mathrm{x}$-ray diffraction. $\mathrm{C} 2, \mathrm{C} 7$ and $\mathrm{C} 10$ were determined to be $(R)$-configuration and $\mathrm{C} 5$ and $\mathrm{C} 6$ were determined to be $(S)$-configuration (see Figure S3). The crystals were grown by slow evaporation and solvent diffusion from dichloromethane/hexane. Although single crystal diffraction is not necessarily representative of the bulk, other techniques such as (CSP-HPLC) have been used to establish the dominance of one enantiomer.

\section{Experimental}

A suitable single crystal of $\mathrm{C}_{20} \mathrm{H}_{18} \mathrm{O}_{5} \mathrm{Cl}_{4}$ [5750] was selected and mounted in NVL Oil in a LithoLoop on a 'Bruker APEX-II CCD' diffractometer. The crystal was kept at $100.0 \mathrm{~K}$ during data collection. Using Olex $2,{ }^{15}$ the structure was solved with the $\mathrm{XS}^{16}$ structure solution program using Direct Methods and refined with the SHELXL ${ }^{17}$ refinement package using Least Squares minimisation.

Crystal Data for $\mathrm{C}_{20} \mathrm{H}_{18} \mathrm{O}_{5} \mathrm{Cl}_{4}(M=480.14 \mathrm{~g} / \mathrm{mol})$ : orthorhombic, space group $\mathrm{P} 2{ }_{1}{ }_{1} 2_{1}$ (no. 19), $a=8.3094(6) \AA, b=14.0415(12) \AA, c=18.0419(16) \AA, V=2105.1(3) \AA^{3}, Z=4, T=$ $100.0 \mathrm{~K}, \mu(\mathrm{MoK} \alpha)=0.592 \mathrm{~mm}^{-1}$, Dcalc $=1.515 \mathrm{~g} / \mathrm{cm}^{3}, 20318$ reflections measured $\left(5.368^{\circ} \leq\right.$ $\left.2 \Theta \leq 56.178^{\circ}\right), 5093$ unique $\left(R_{\text {int }}=0.0527, \mathrm{R}_{\text {sigma }}=0.0541\right)$ which were used in all calculations . The final $R_{1}$ was $0.0711(\mathrm{I}>2 \sigma(\mathrm{I}))$ and $w R_{2}$ was 0.1857 (all data).

Crystal data have been deposited at the Cambridge Crystallographic Data Centre with the deposition number CCDC 1946937. 
Displacement ellipsoid plot of 5a with ellipsoids drawn at the 50\% probability level and $\mathrm{H}$ atoms shown with spheres of arbitrary radii.

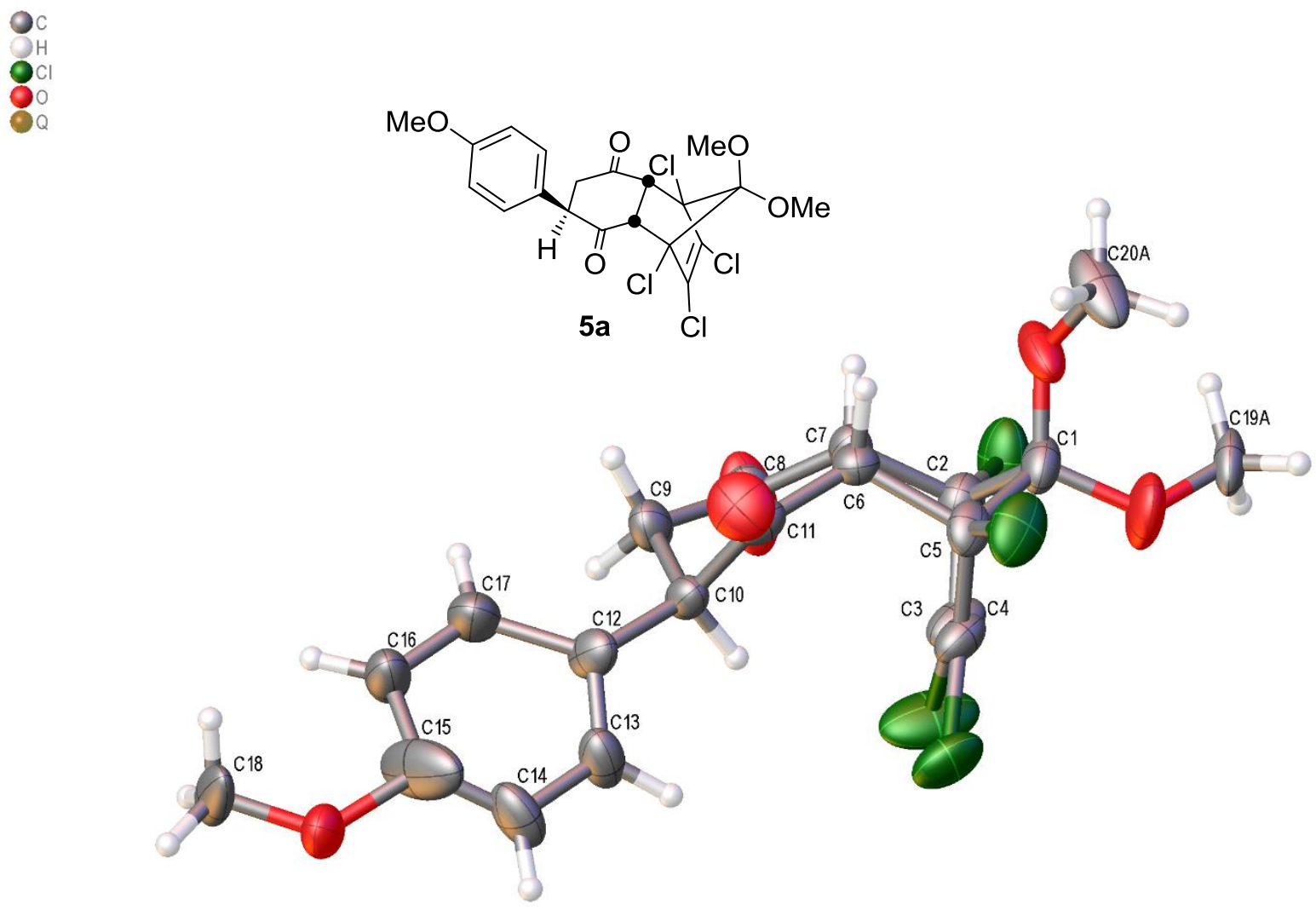

Figure S3: Crystal structure and chemdraw structure of product 5a

Table S-3: Crystal data and structure refinement for 5750.

Identification code

5750

Empirical formula

$\mathrm{C}_{20} \mathrm{H}_{18} \mathrm{O}_{5} \mathrm{Cl}_{4}$

Formula weight

480.14

Temperature/K

100.0

Crystal system

orthorhombic

Space group

$\mathrm{P} 2{ }_{1} 2{ }_{1}{ }_{1}$

$\mathrm{a} / \AA$

$8.3094(6)$

$\mathrm{b} / \AA$

14.0415(12)

$\mathrm{c} / \AA$

18.0419(16)

$\alpha /{ }^{\circ}$

90

$\beta /{ }^{\circ}$

90

$\gamma /{ }^{\circ}$

90

Volume/ $/ \AA^{3}$

2105.1(3)

Z

4

$\rho_{\text {calc }} \mathrm{g} / \mathrm{cm}^{3}$

1.515

$\mu / \mathrm{mm}^{-1}$

0.592 
$\mathrm{F}(000)$

984.0

Crystal size $/ \mathrm{mm}^{3}$

$0.52 \times 0.42 \times 0.18$

Radiation

$\operatorname{MoK} \alpha(\lambda=0.71073)$

$2 \Theta$ range for data collection/ ${ }^{\circ} 5.368$ to 56.178

Index ranges

$-10 \leq \mathrm{h} \leq 6,-18 \leq \mathrm{k} \leq 18,-23 \leq 1 \leq 23$

Reflections collected

20318

Independent reflections

$5093\left[\mathrm{R}_{\text {int }}=0.0527, \mathrm{R}_{\text {sigma }}=0.0541\right]$

Data/restraints/parameters

$5093 / 36 / 324$

Goodness-of-fit on $\mathrm{F}^{2}$

1.044

Final $R$ indexes $[\mathrm{I}>=2 \sigma(\mathrm{I})] \quad \mathrm{R}_{1}=0.0711, \mathrm{wR}_{2}=0.1656$

Final $\mathrm{R}$ indexes [all data] $\quad \mathrm{R}_{1}=0.1042, \mathrm{wR}_{2}=0.1857$

Largest diff. peak/hole / e $\AA^{-3} 0.74 /-0.86$

Flack parameter $\quad 0.02(4)$

\section{References:}

1. K. Kikushima, J. C. Holder, M. Gatti and B. M. Stoltz, J. Am. Chem. Soc., 2011, 133, 6902-6905.

2. A. J. J. Lennox and G. Lloyd-Jones, Chem. Soc. Rev., 2014, 43, 412.

3. J. C. Holder, A. N. Marziale, P. Liu, Y. Lan, M. Gatti, K. Kikushima, K. N. Houk and M. Stoltz, J. Am. Chem. Soc., 2013, 135, 14996-15007.

4. D. Bancroft, P., F. A. Cotton and M. Verbruggen, Acta Crystallogr., 1989, C45, 1289.

5. R. Sarkar and S. Mukherjee, Org. Lett., 2016, 18, 6160.

6. P. Blandon, S. McVey, P. L. Pauson, G. D. Broadhead and M. W. Horspool, J. Chem. Soc. (C), 1966, 306.

7. J. M. Coxon, J. O'Connell and P. J. Steel, Aust. J. Chem., 1986, 39, 1537.

8. J.-S. Yang, H.-H. Huang and S.-H. Lin, J. Org. Chem., 2009, 74, 3974.

9. J. B. Maccaulay and A. G. Fallis, J. Am. Chem. Soc., 1990, 112, 1136.

10. Z. Zhu and J. H. Espenson, J. Am. Chem. Soc., 1997, 119, 3507.

11. H. Adams, S. Jones and I. Ojea-Jimenez, Org. Biomol. Chem., 2006, 4, 2296.

12. M. Oda, T. Kawase, T. Okada and T. Enomoto, Org. Synth., 1996, 73, 253.

13. K. Aikawa, T. Okamoto and K. Mikami, J. Am. Chem. Soc., 2012, 134, 10329.

14. G. Y. Wooi and J. M. White, Org. Biomol. Chem., 2005, 3, 972.

15. O. V. Dolomanov, L. J. Bourhis, R. J. Gildea, J. A. K. Howard and H. Puschmann, J. Appl. Crystallogr., 2009, 42, 339.

16. G. M. Sheldrick, Acta Crystallogr., 2008, A64, 112.

17. G. M. Sheldrick, Acta Crystallogr., 2015, A71, 3. 


\section{${ }^{1} \mathrm{H}$ and ${ }^{13} \mathrm{C}$ NMR}

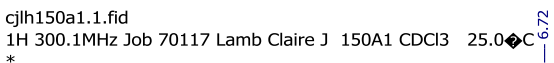

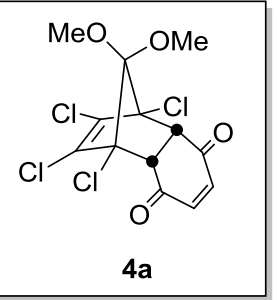

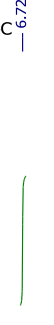
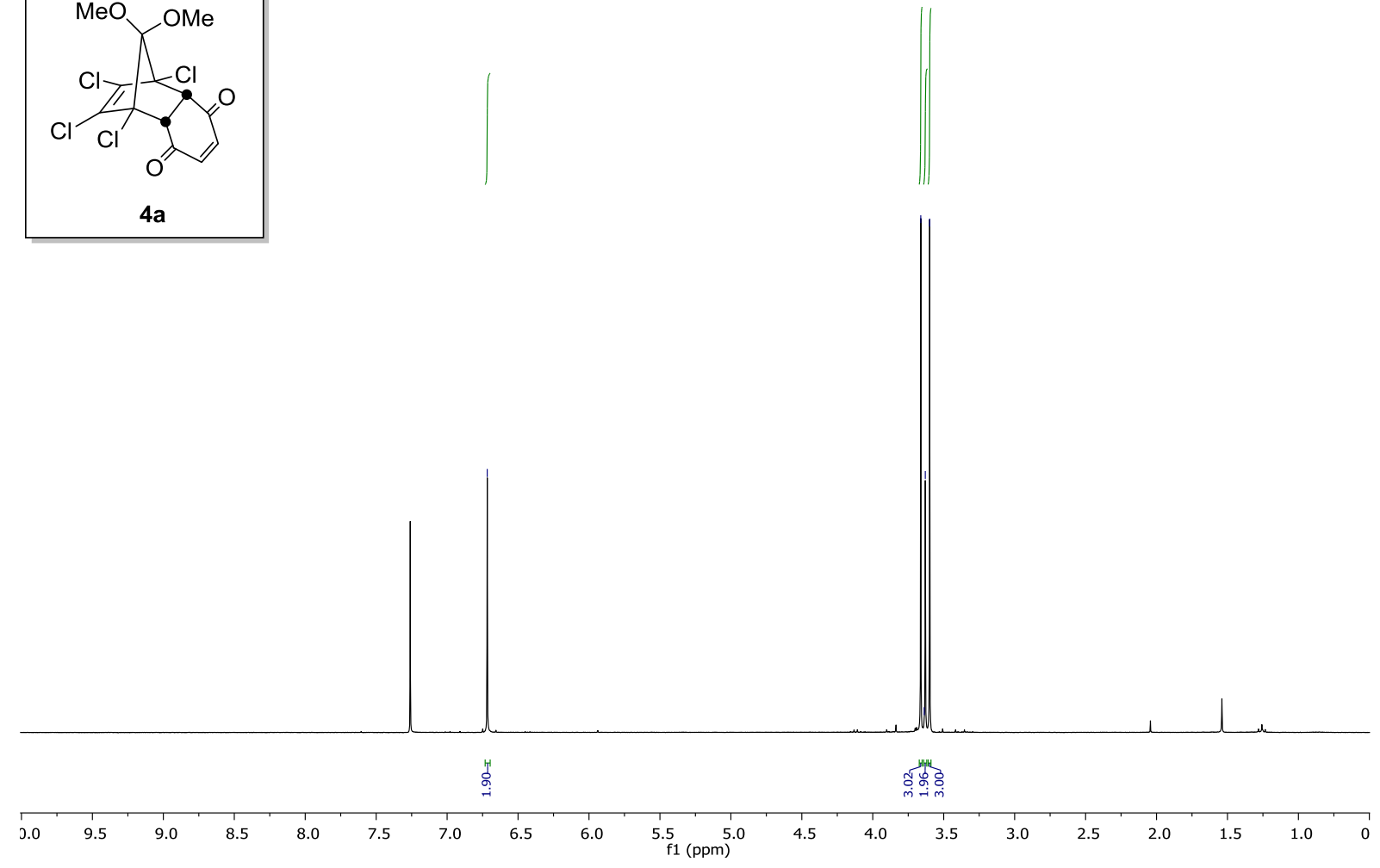

cjlc150a1.1.fid

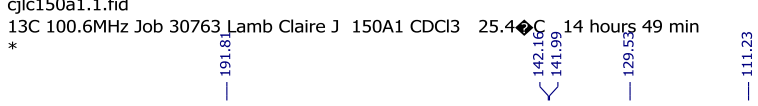
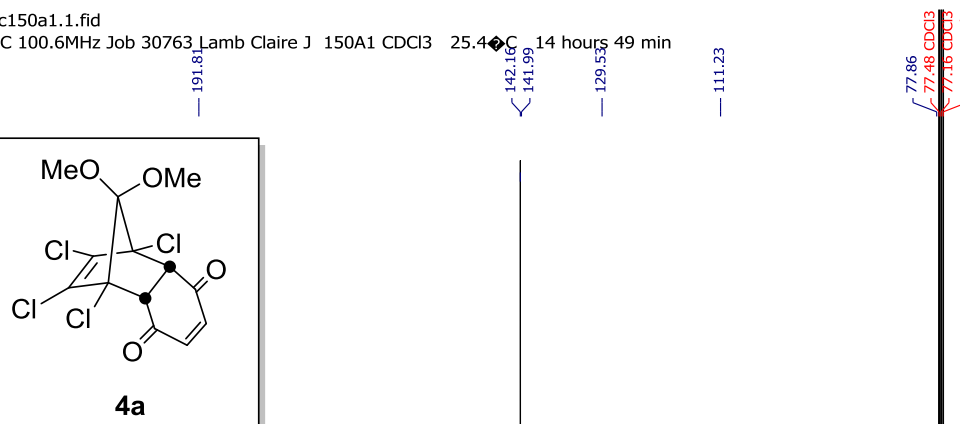


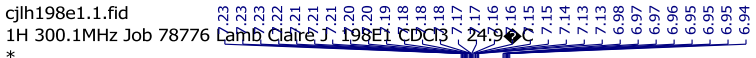
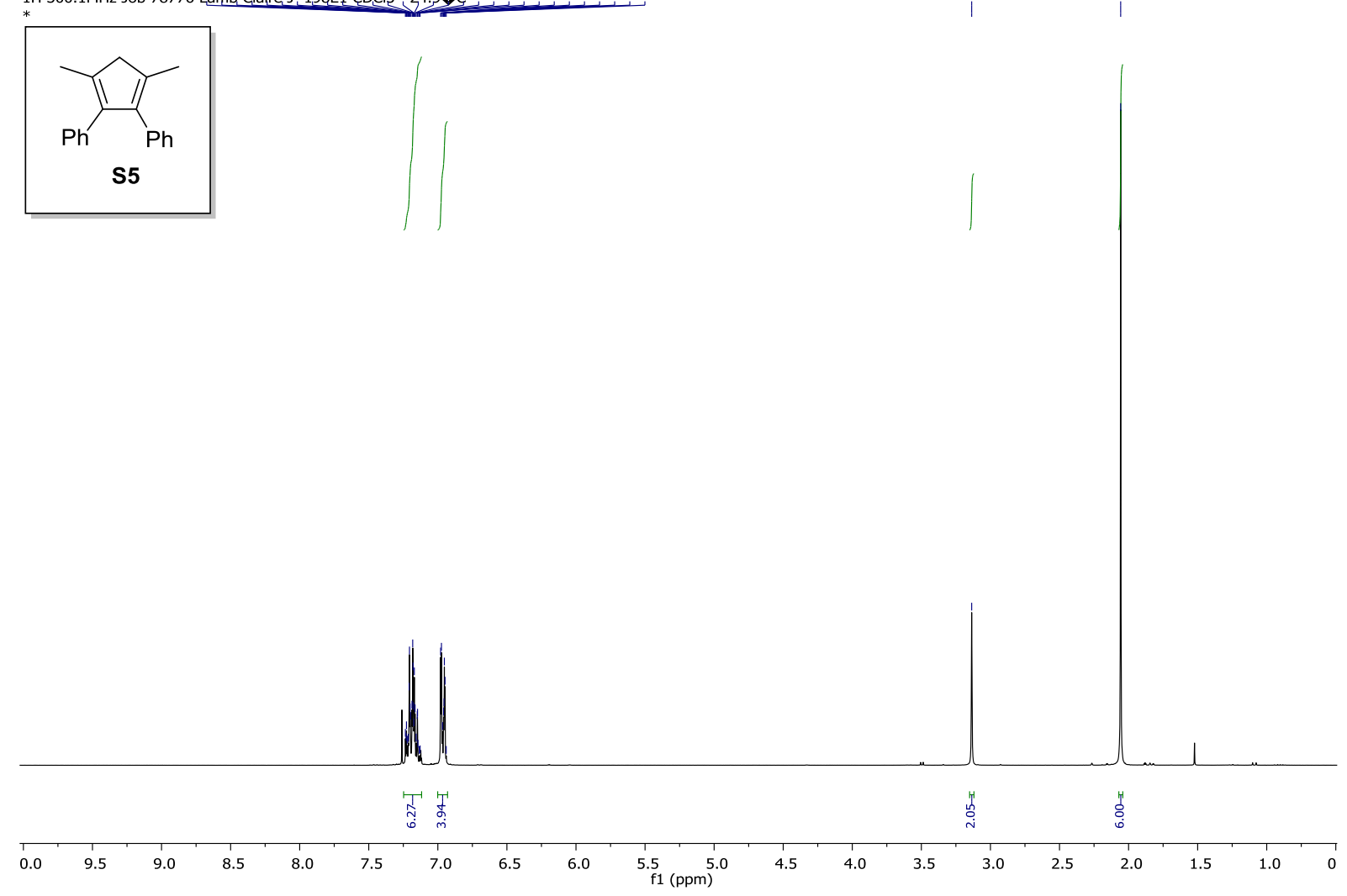

cjlc198e1.1.fid

13C 75.5MHz Job 78800 Lamb Claire J 198E1 CDCl3 25.0 C 2 h hours 7 min

*

证

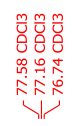

$\stackrel{m}{7}$

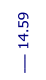

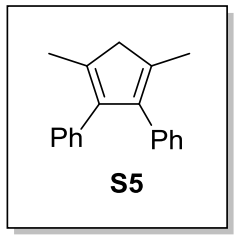


cjlh200b1.1.fid

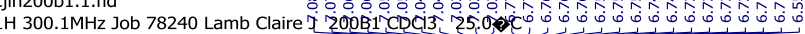

$$
\text { (ne }
$$

$4 \mathbf{b}$
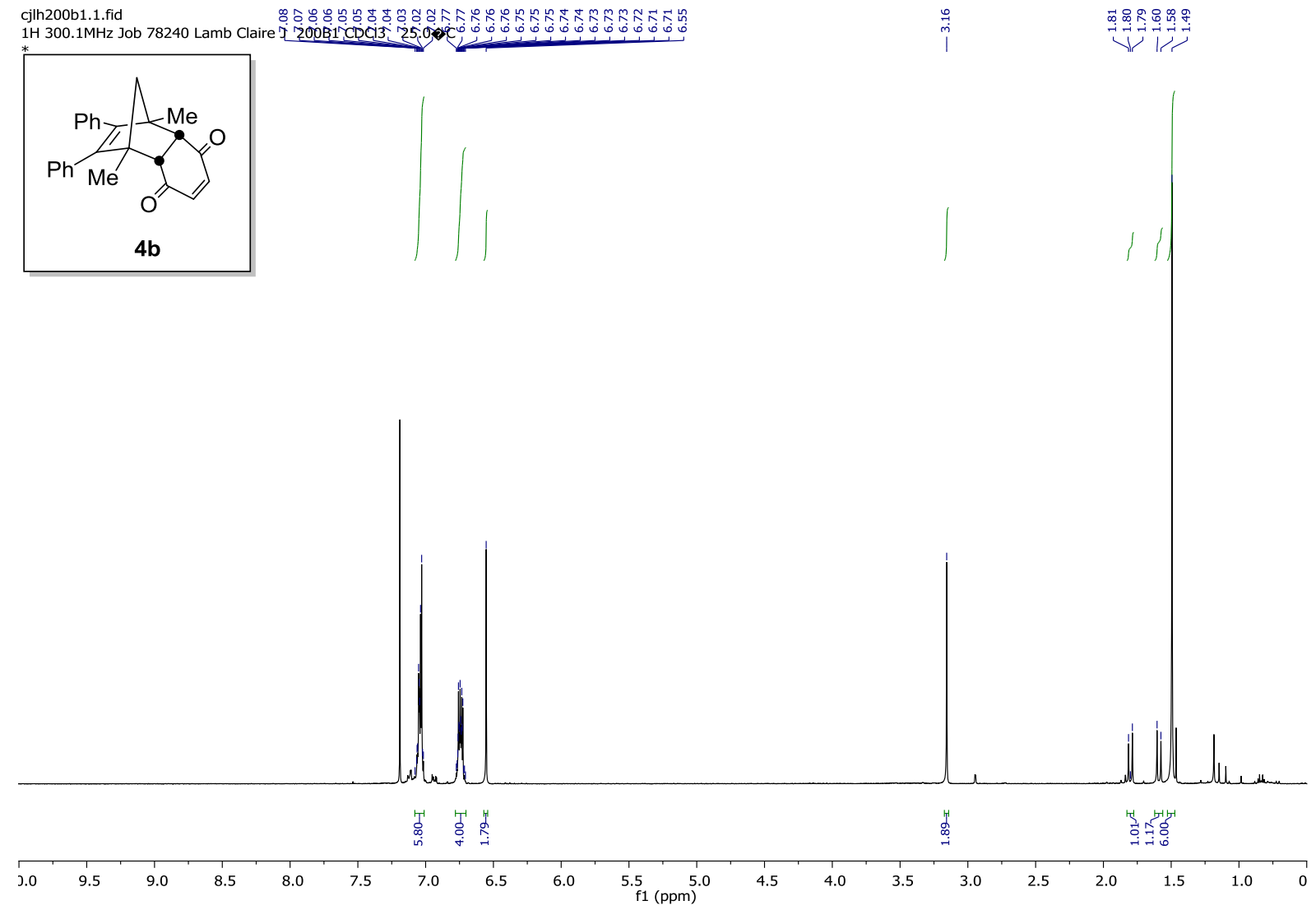

cjlc200b1.1.fid

13C 75.5MHz Job 78313 Lamb Claire J 200B1 CDCl3 25.0 C 3 hgurs 1 min

*

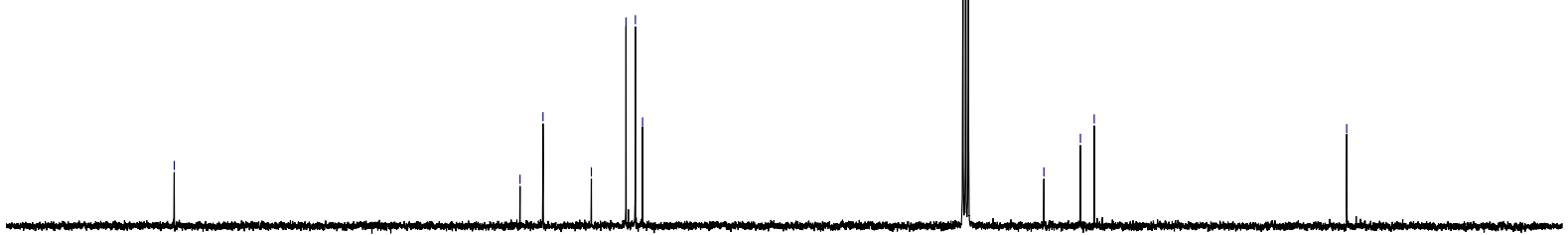

$\begin{array}{llllllllllll}220 & 210 & 200 & 190 & 180 & 170 & 160 & 150 & 140 & 130 & 120 & \begin{array}{c}110 \\ \mathrm{f} 1(\mathrm{ppm})\end{array}\end{array}$ 


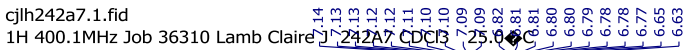

$$
\text { (ne) }
$$

4c
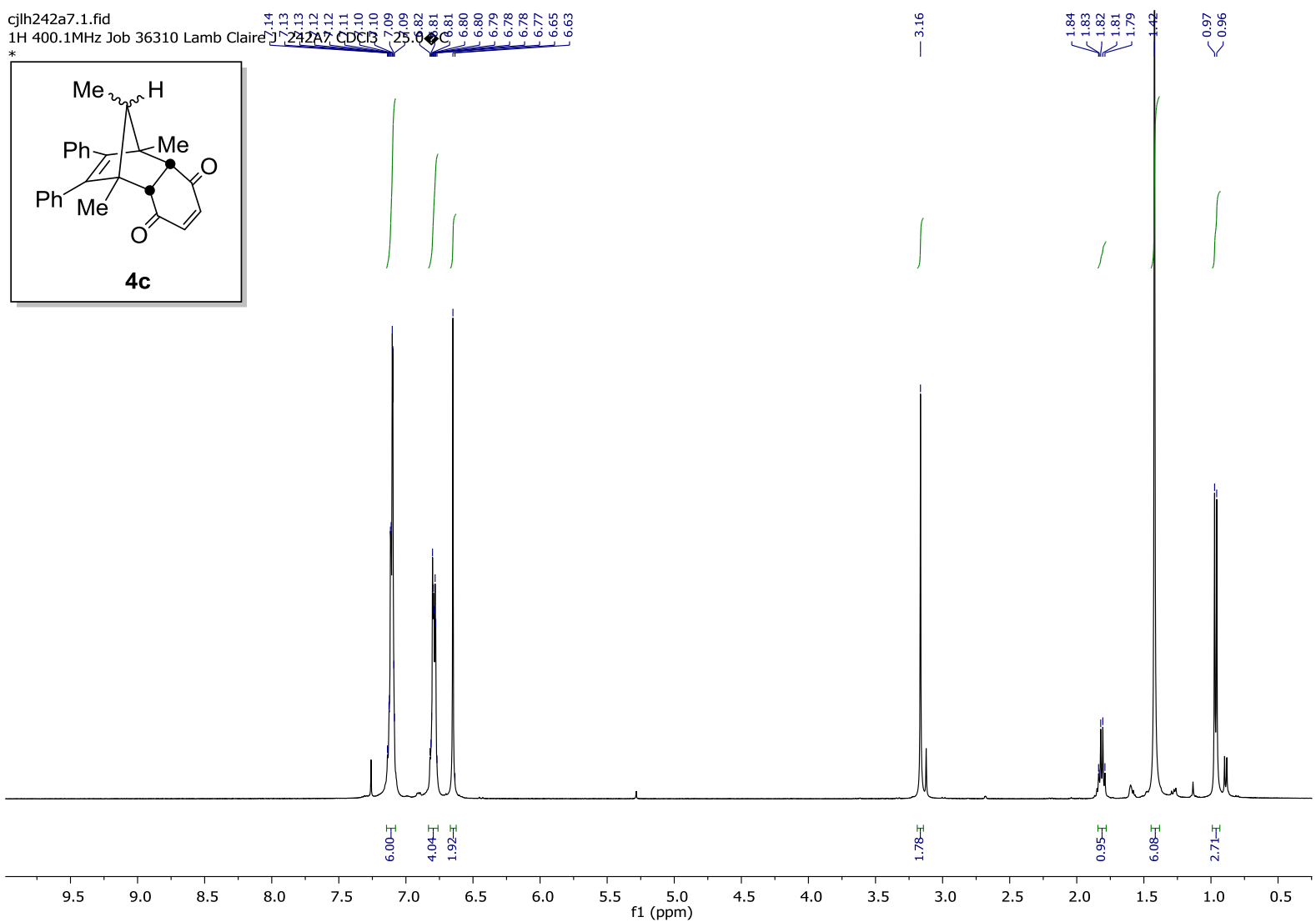

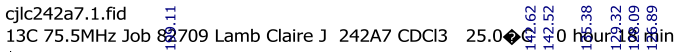

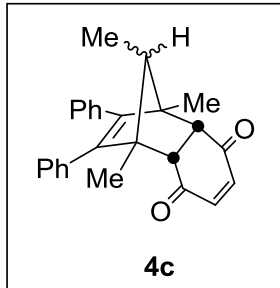

$\begin{array}{llllllllllll}220 & 210 & 200 & 190 & 180 & 170 & 160 & 150 & 140 & 130 & 120 & 110 \begin{array}{l}100 \\ f 1(\mathrm{ppm})\end{array}\end{array}$ 


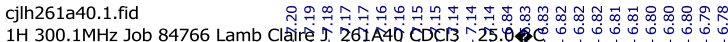
IH $300.1 \mathrm{MHz}$
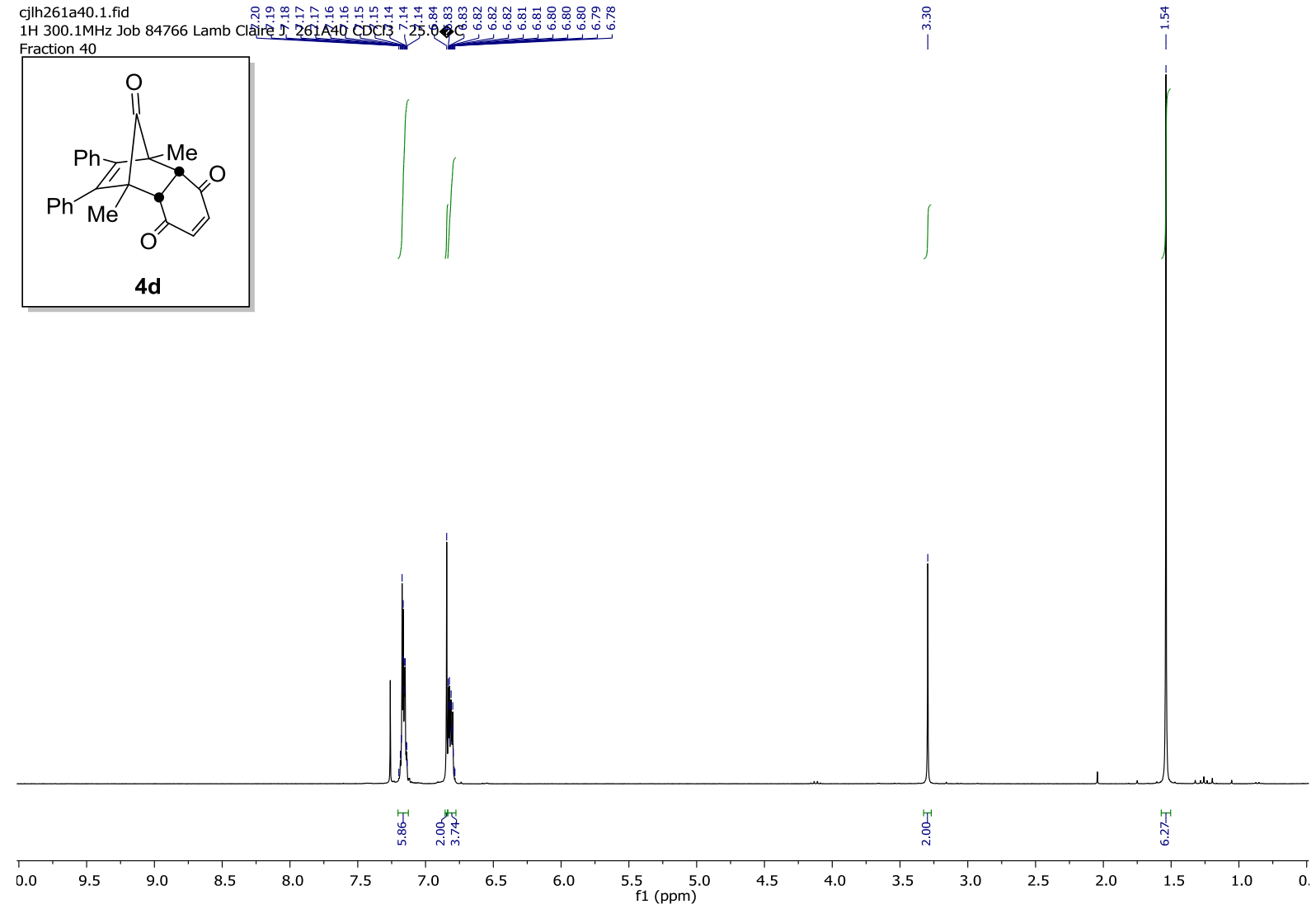
cjlc261a40.1.fid

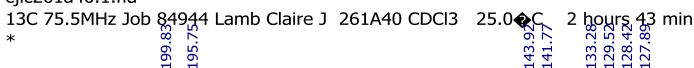
$\stackrel{\text { । }}{\text { । }}$

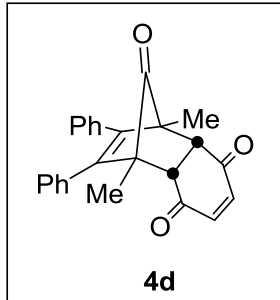

$\begin{array}{lllllllllllll}220 & 210 & 200 & 190 & 180 & 170 & 160 & 150 & 140 & 130 & 120 & 110 & 100 \\ \mathrm{f} 1(\mathrm{ppm})\end{array}$ 


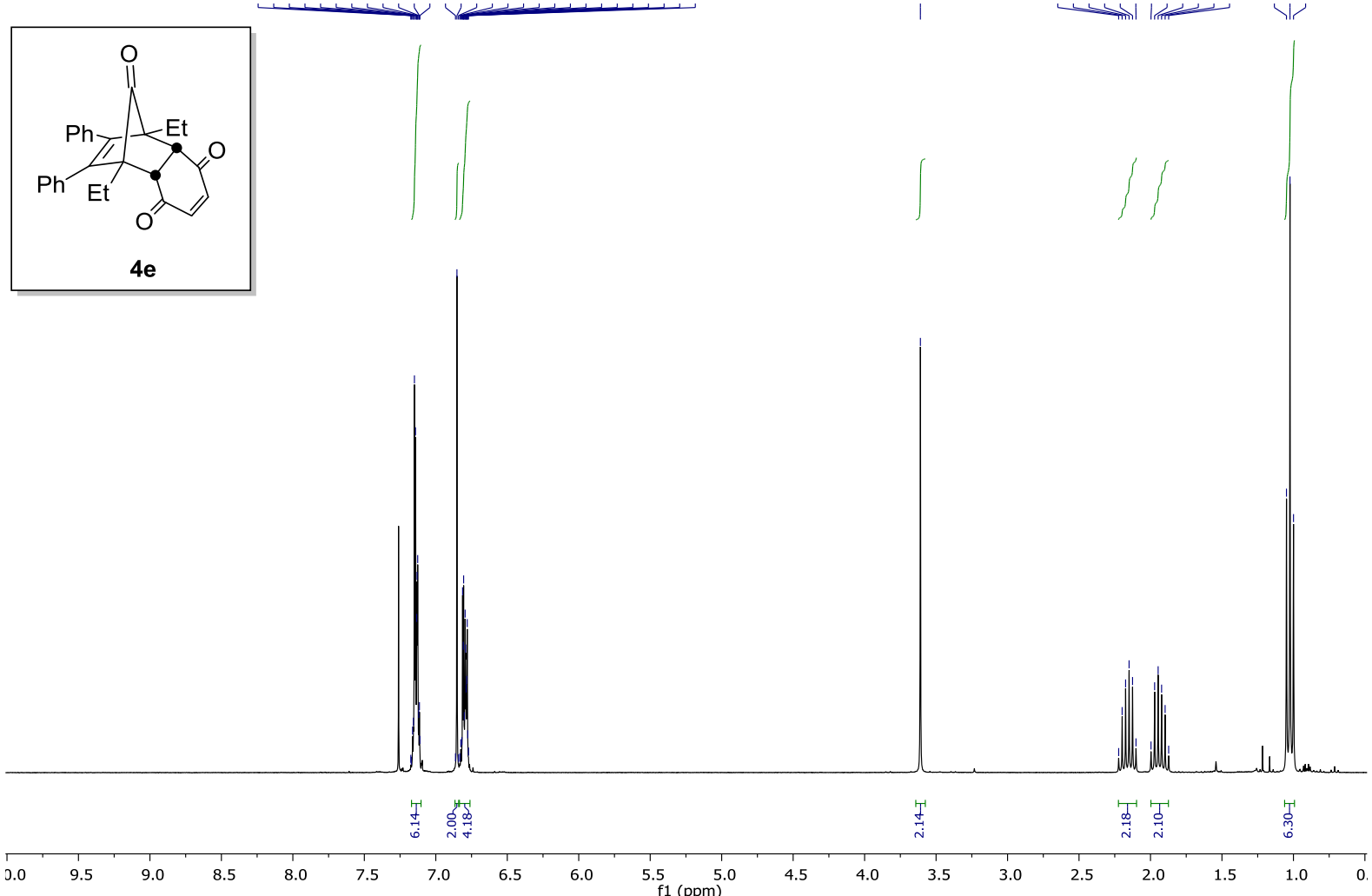

cjlc263a1p2.1.fid

13C 75.5MHz Job 85078 Lamb Claire J 263A1P2 CDCl3 25.0 2 \& hours 1 min

* 13 7.5MHz Job

।

1/

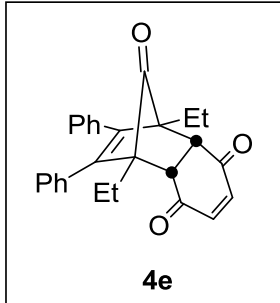

$4 e$

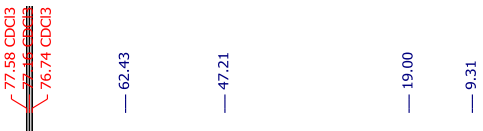

$\begin{array}{llllllllllll}220 & 210 & 200 & 190 & 180 & 170 & 160 & 150 & 140 & 130 & 120 & \begin{array}{c}110 \\ \mathrm{f} 1(\mathrm{ppm})\end{array}\end{array}$ 
cjlh253ab1.1.fid

1H 400.1MHz Job 36674 Lamb Claire J 253AB1 CDCl3 24.9॰C

守
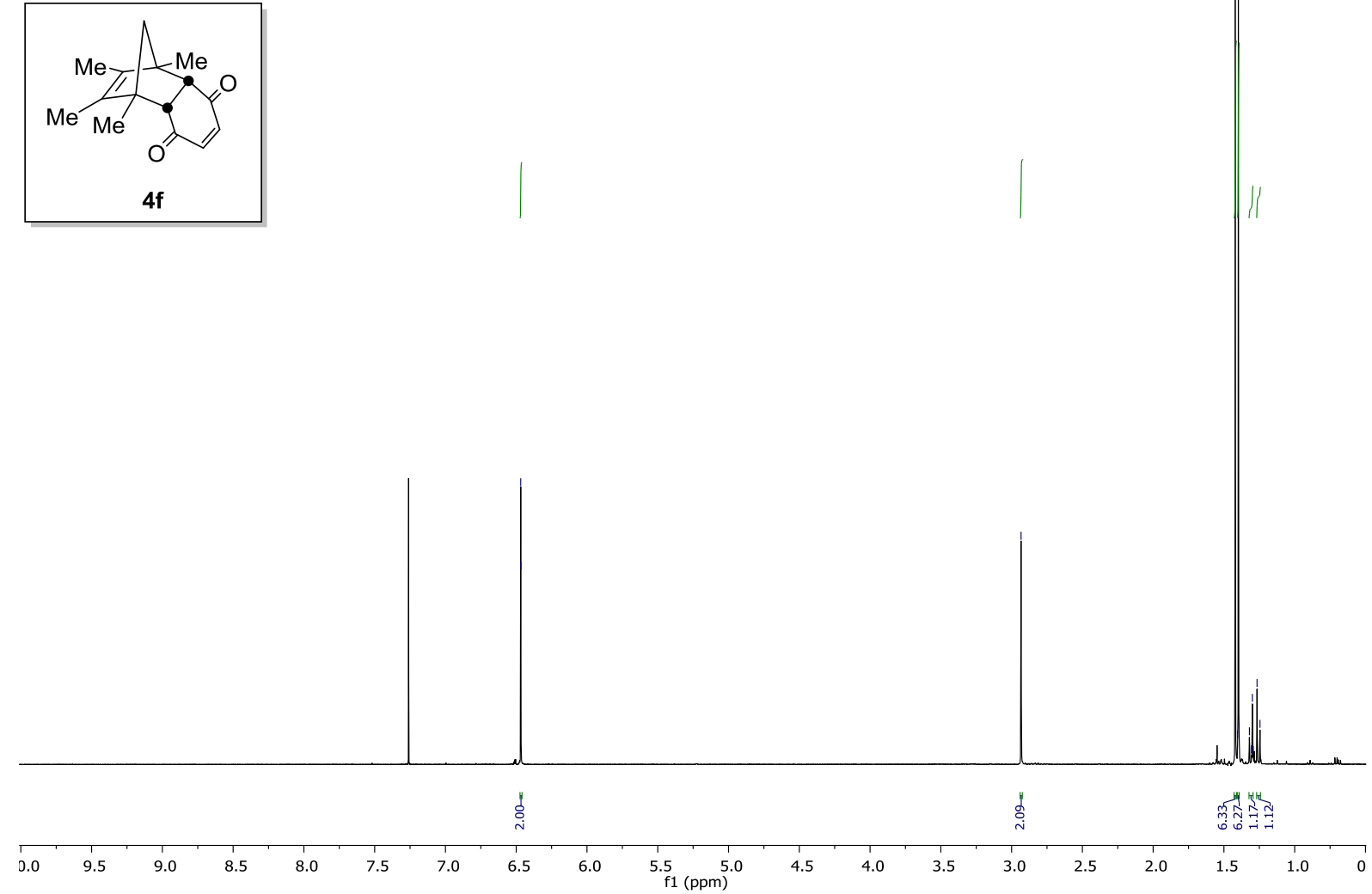

cjlc253ab1.1.fid

13C 100.6MHz Job 36699 Lamb Claire J 253AB1 CDCl3 25.4^ 4 hours $22 \mathrm{~min}$

|
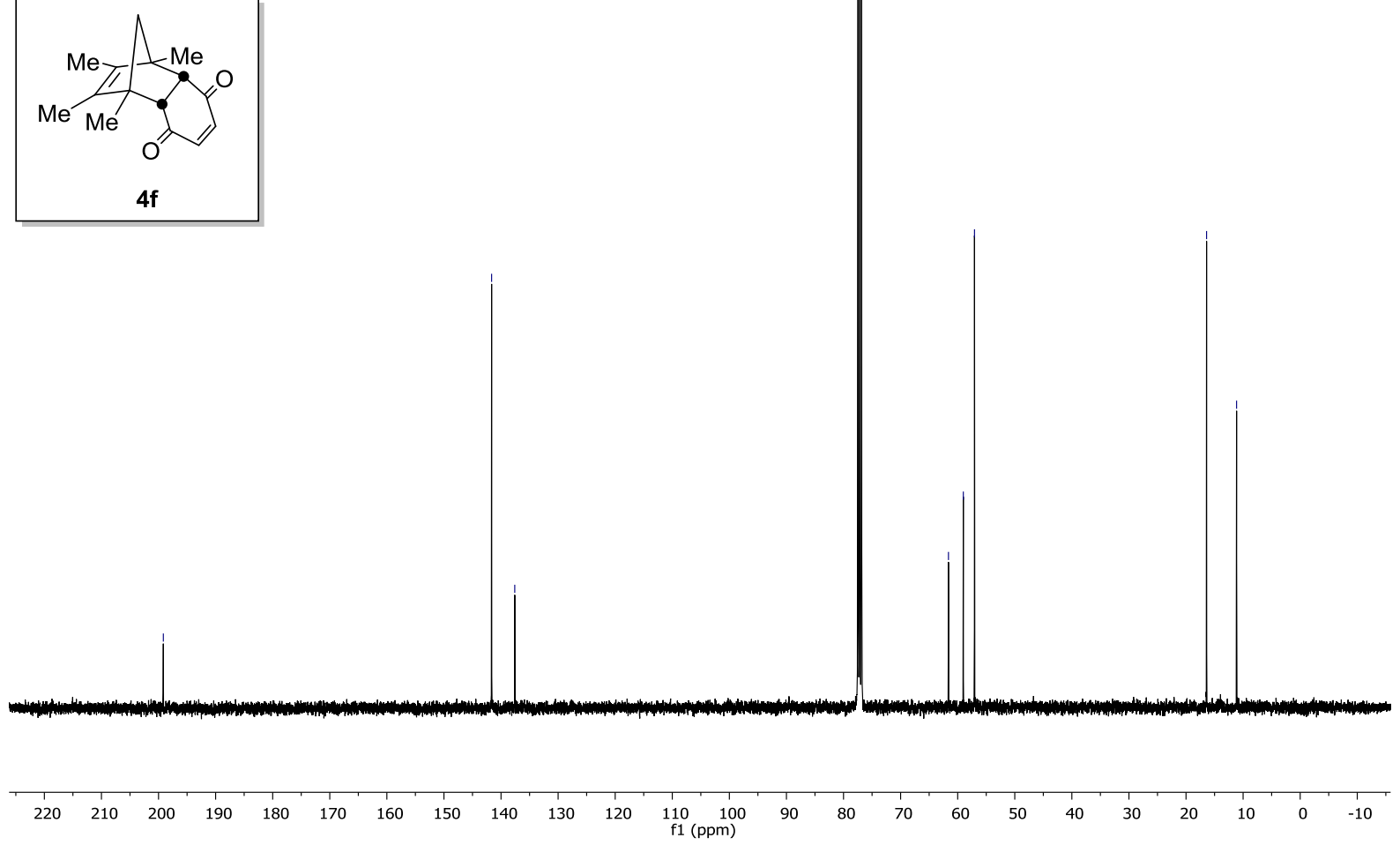
cjlh250abb1.1.fid

$1 \mathrm{H} 300.1 \mathrm{MHz}$ Job 83930 Lamb Claire J 250ABB1 CDCl3 $24.9 \gg \mathrm{C}^{6}$
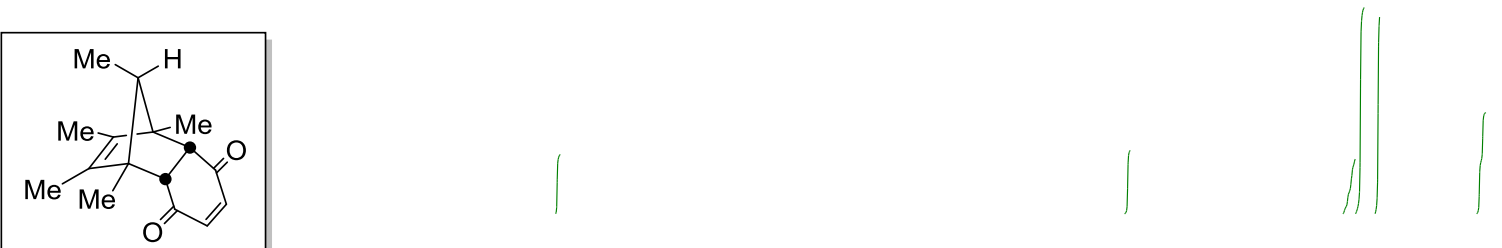

4g

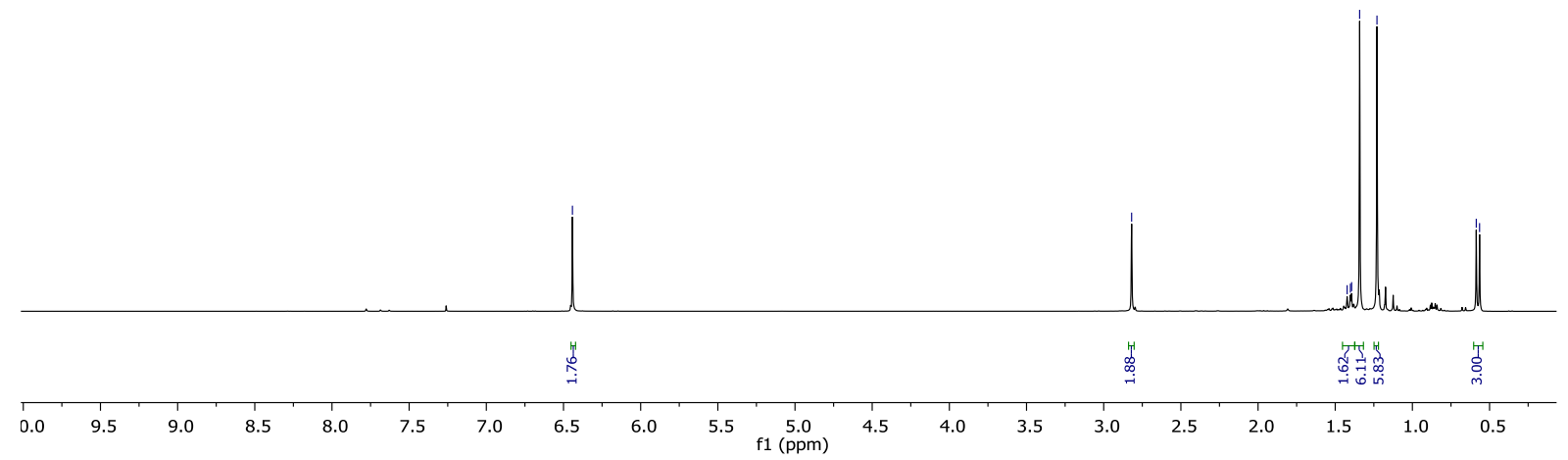

cjlc250ab.1.fid

13C 75.5MHz Job 83965 Lamb Claire J $250 \mathrm{AB}$ CDCl3 $25.0 \gg \mathrm{C} 3$ hqurs $1 \mathrm{~min}$

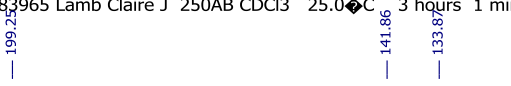

商旁品

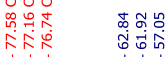

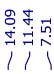
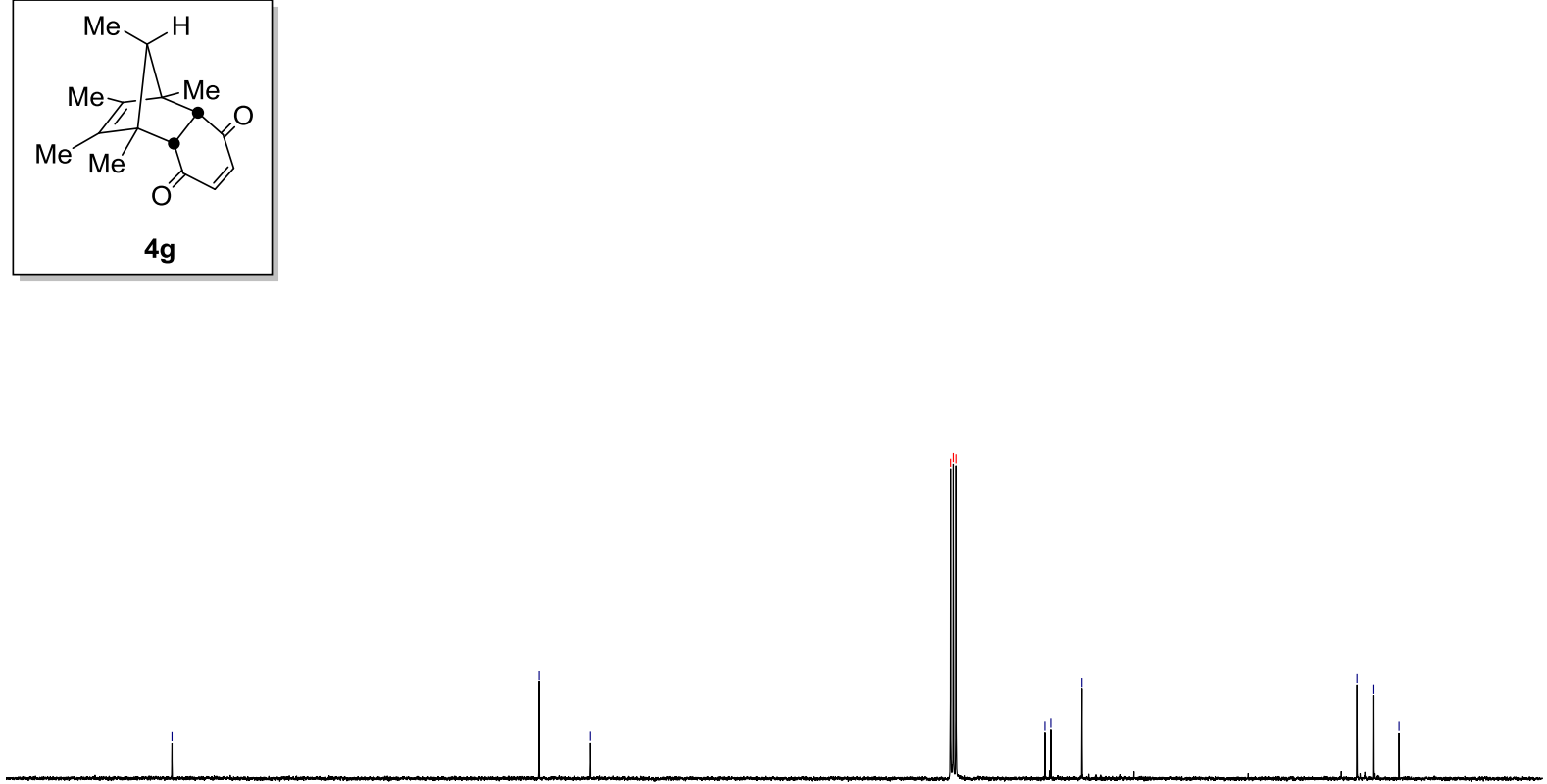

$\begin{array}{llllllllllll}220 & 210 & 200 & 190 & 180 & 170 & 160 & 150 & 140 & 130 & 120 & 110 \\ \mathrm{f} 1(\mathrm{ppm})\end{array}$ 


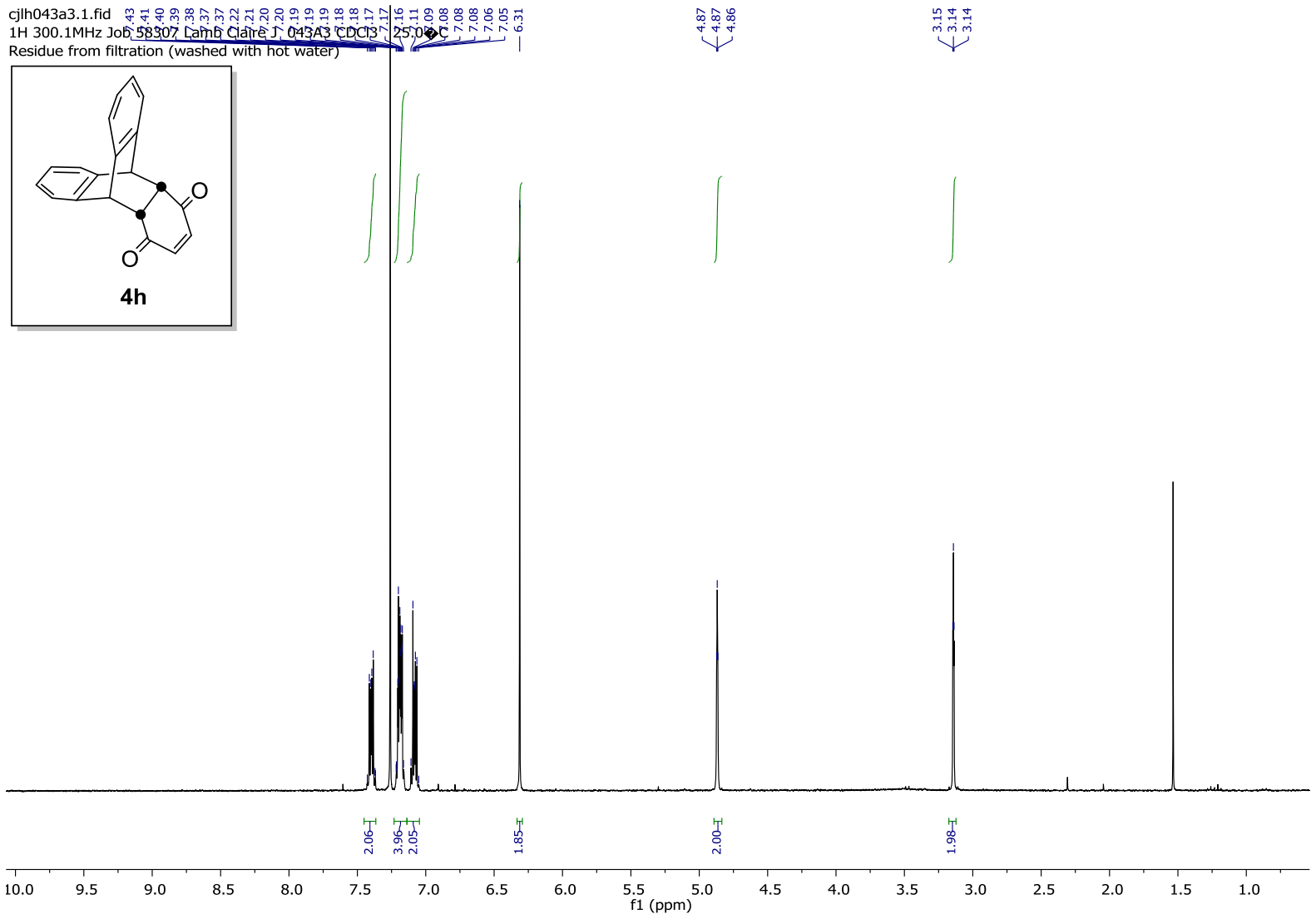

cjlc043b1.1.fid

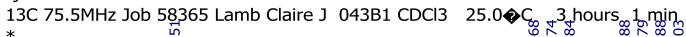

* $\quad$ |
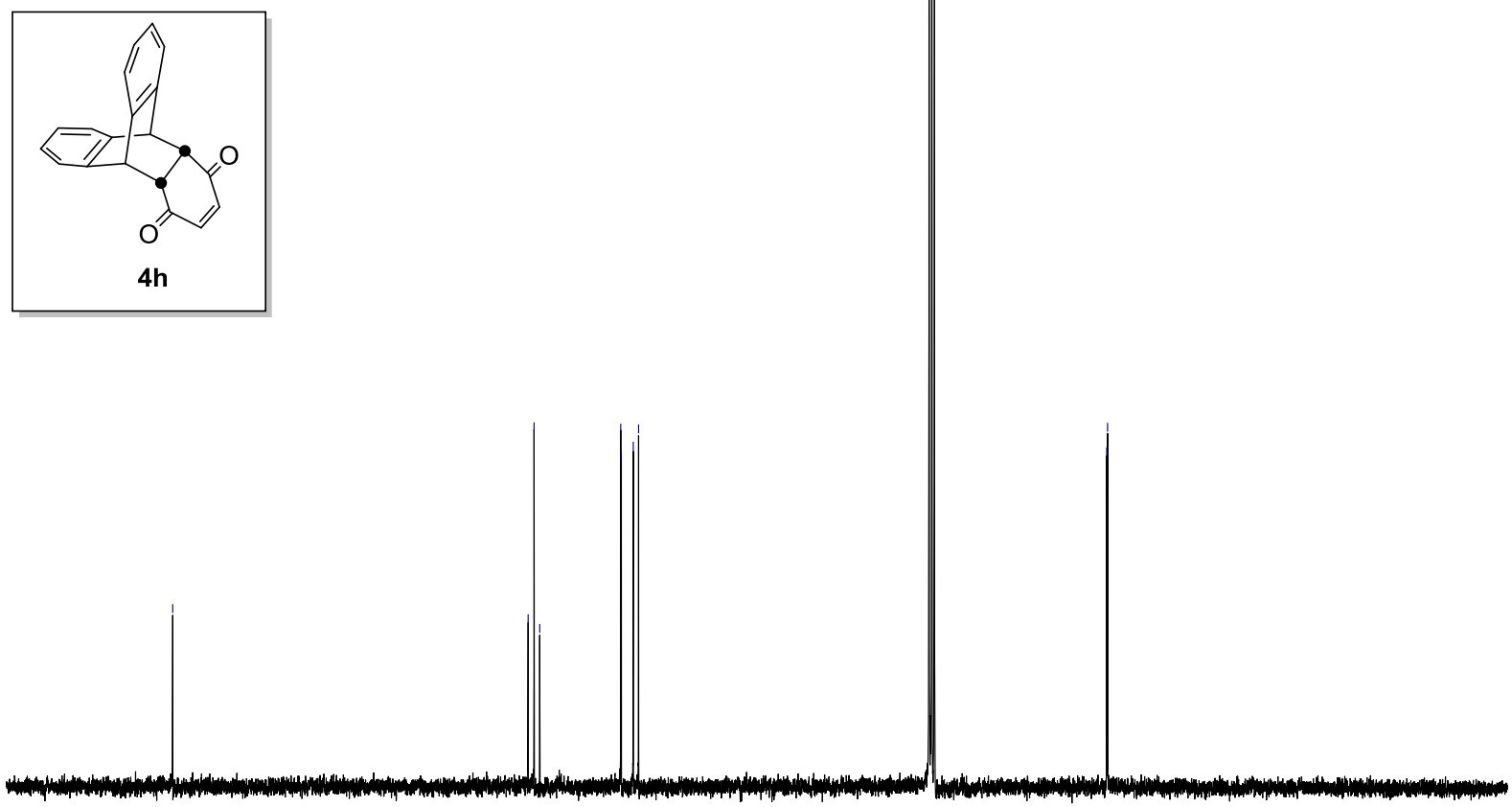

$\begin{array}{llllllllllll}220 & 210 & 200 & 190 & 180 & 170 & 160 & 150 & 140 & 130 & 120 & \begin{array}{l}110 \\ \mathrm{f} 1(\mathrm{ppm})\end{array}\end{array}$ 
cjlh011b.1.fid

1H 400.1MHz Job 28363 Lamb Claire J 011B CDCl3 25.0»C
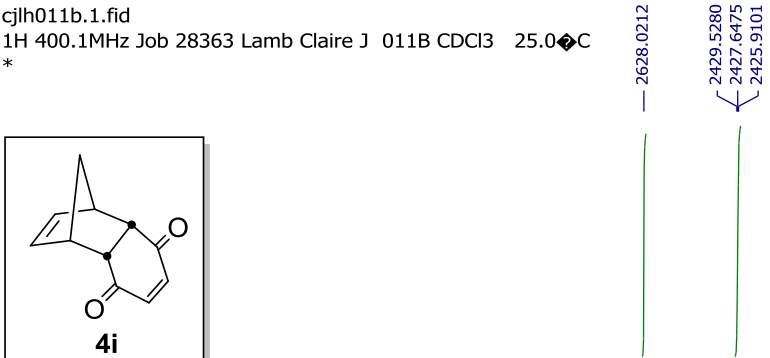

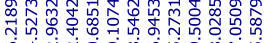

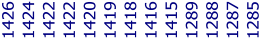

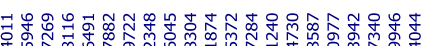

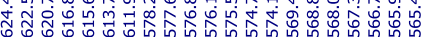

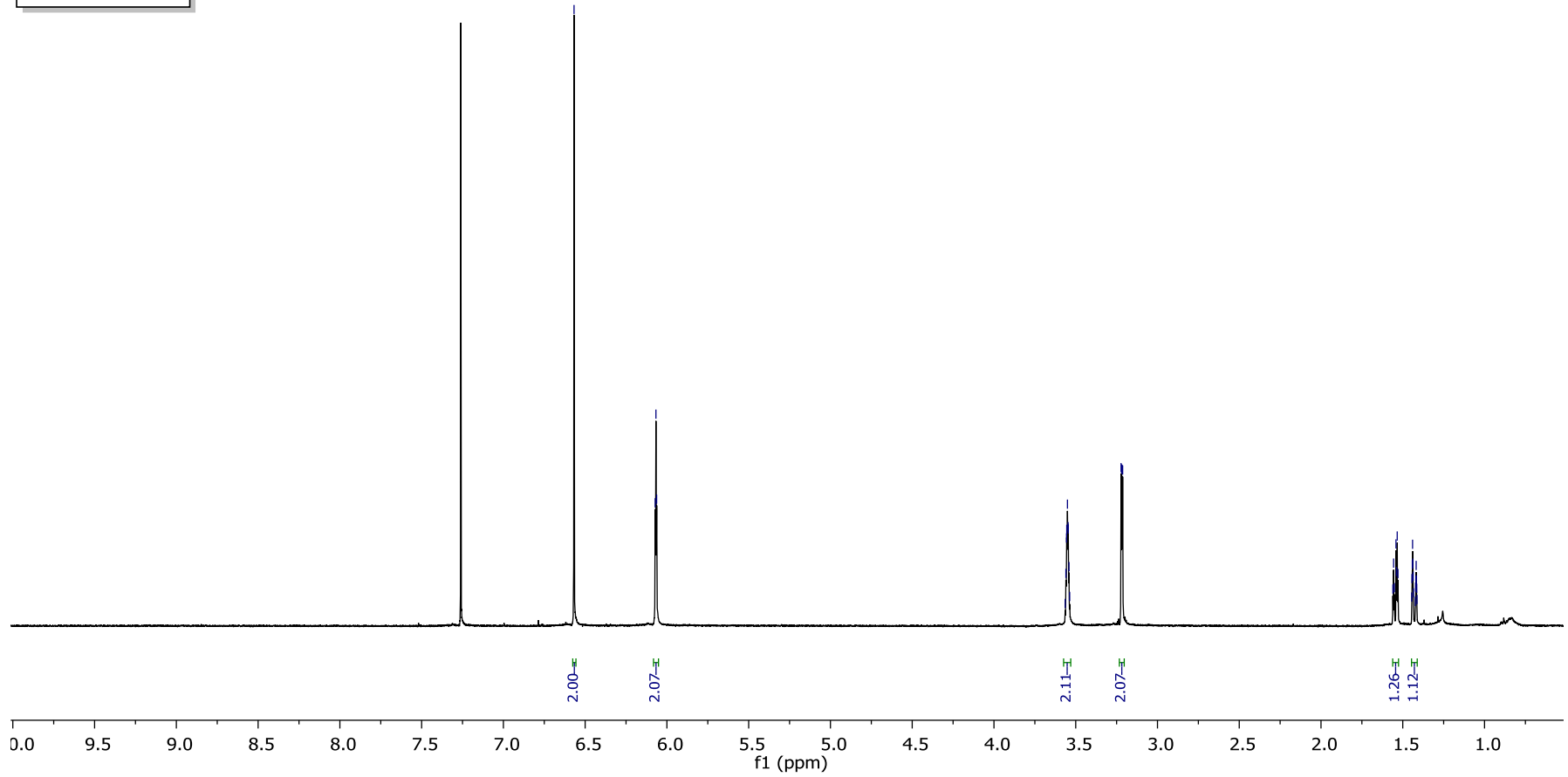

cjlc011a1.1.fid

13C 75.5MHz Job 56290 Lamb Claire J $011 \mathrm{~A} 1 \mathrm{CDCl} 325.0 \gg \mathrm{C}_{\sigma} 3$ hours $1 \mathrm{~min}$ Reaction $011 \mathrm{~A}$
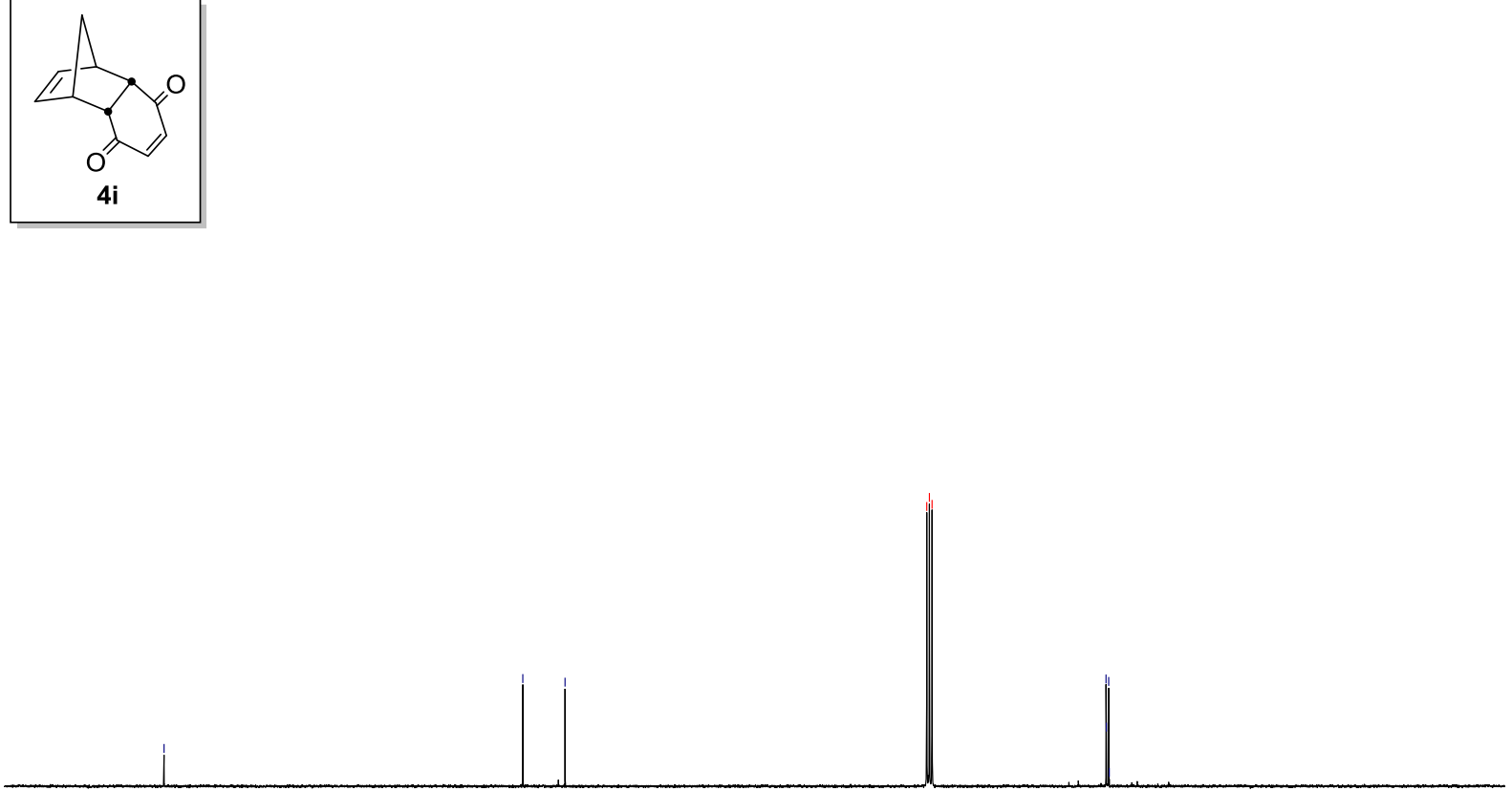

$\begin{array}{llllllllllll}220 & 210 & 200 & 190 & 180 & 170 & 160 & 150 & 140 & 130 & 120 & \begin{array}{c}110 \\ \mathrm{f} 1(\mathrm{ppm})\end{array}\end{array}$ 
cjlh264b4.1.fid

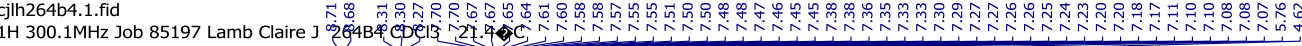
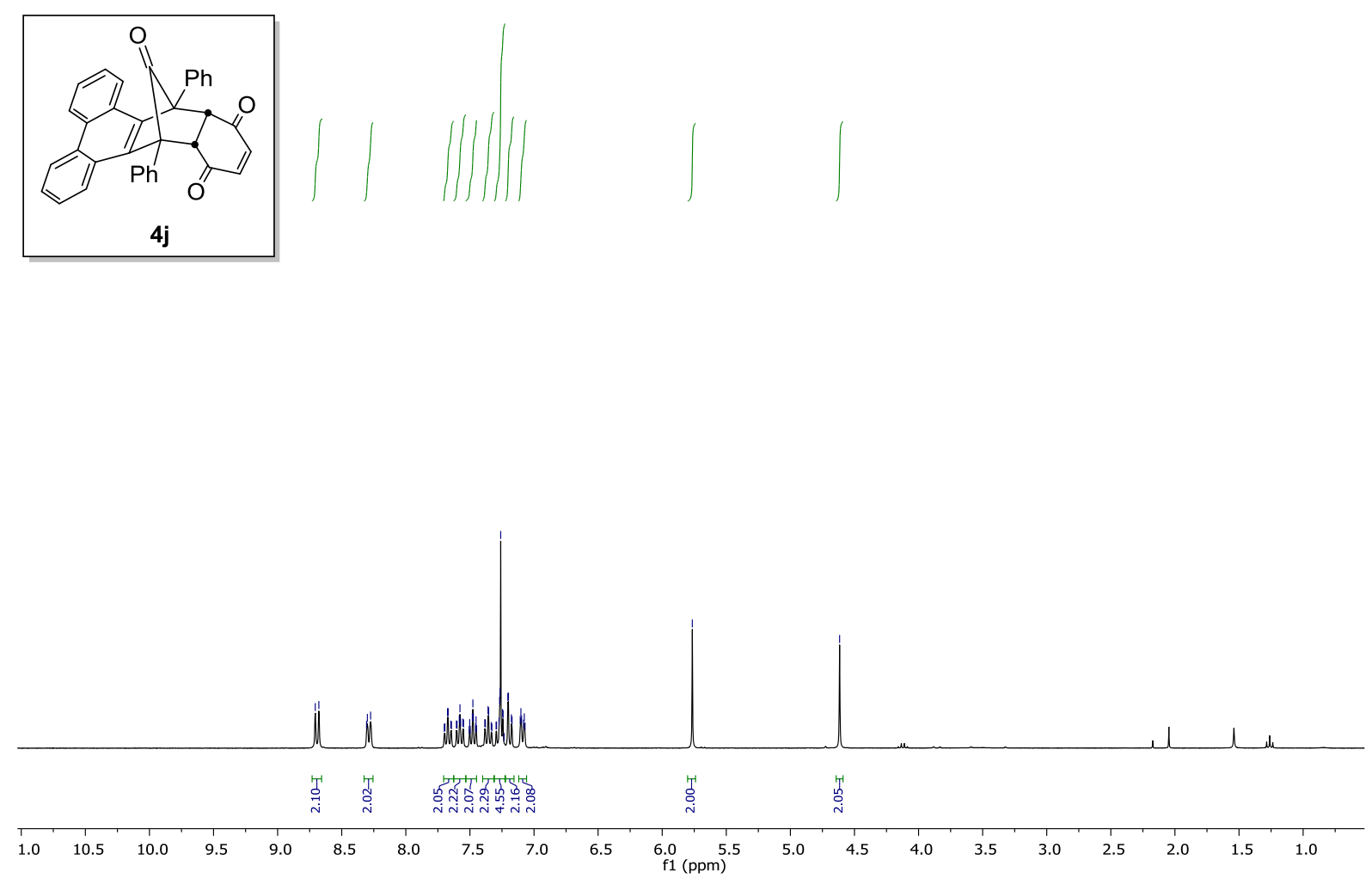

cjlc264a.1.fid

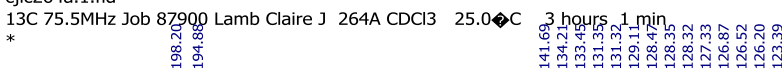

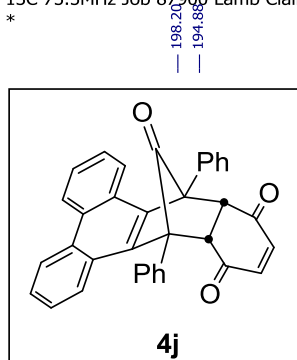

1 परे
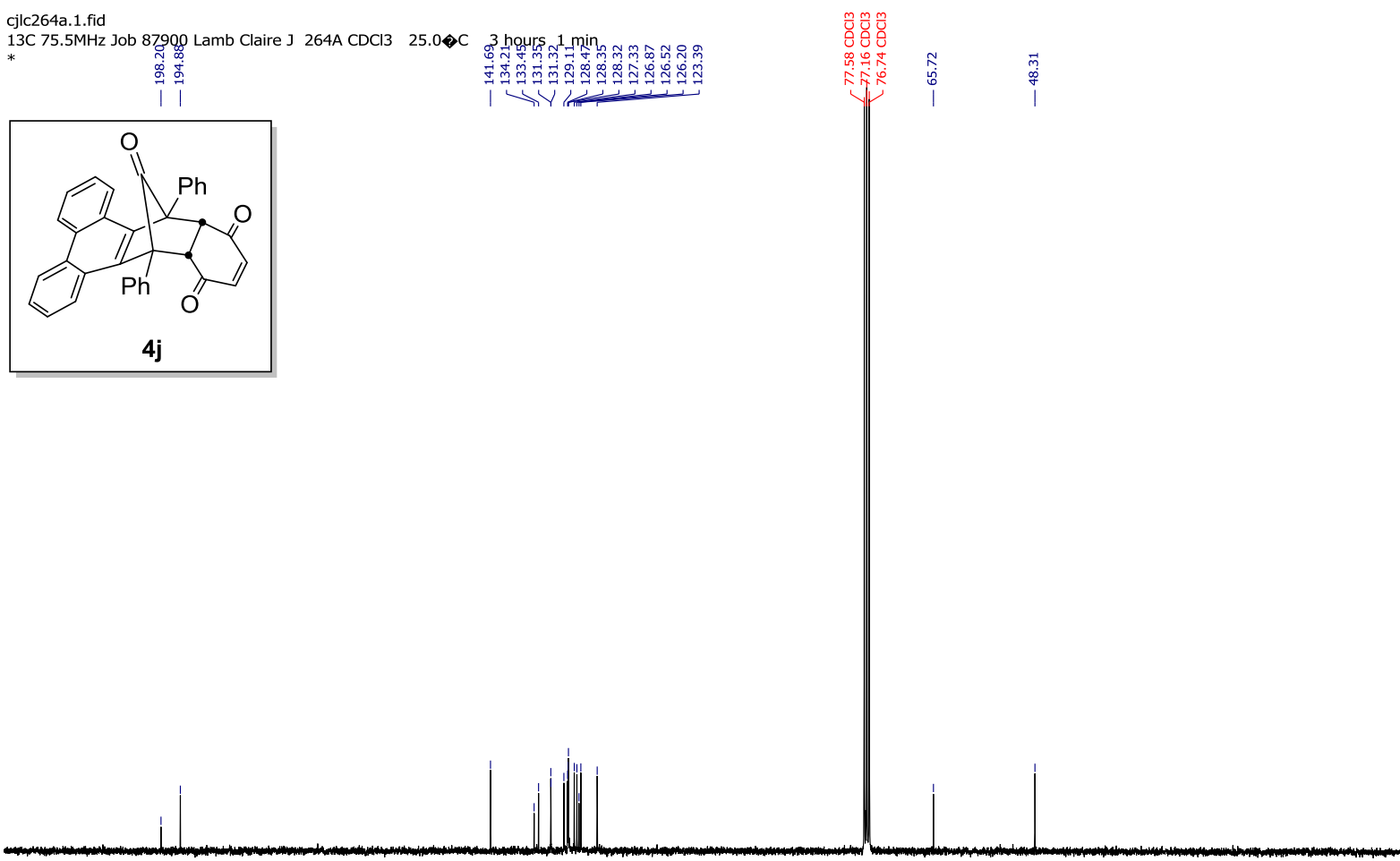

$\begin{array}{llllllllllll}220 & 210 & 200 & 190 & 180 & 170 & 160 & 150 & 140 & 130 & 120 & \begin{array}{c}110 \\ \mathrm{f} 1(\mathrm{ppm})\end{array}\end{array}$ 
cjlh140a2.1.fid S10
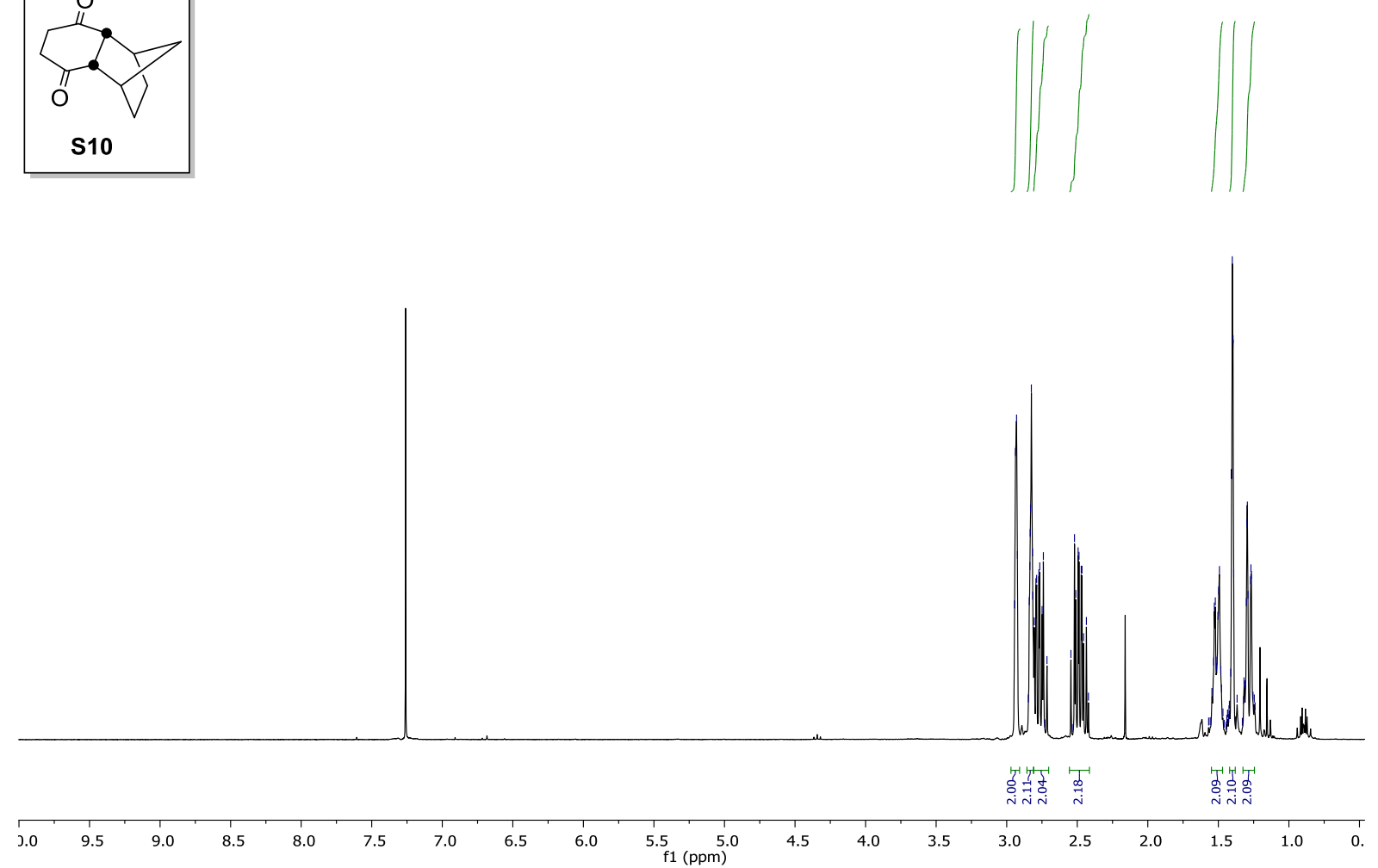

cjlc140a1a.1.fid

13C 100.6M Hz Job 30730 Lamb Claire J 140A1A CDCl3 $25.6 \diamond \mathrm{C} \quad 1$ hour $27 \mathrm{~min}$ $*$

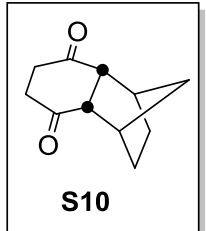

$\begin{array}{llllllllllll}220 & 210 & 200 & 190 & 180 & 170 & 160 & 150 & 140 & 130 & 120 & \begin{array}{c}110 \\ \mathrm{f} 1(\mathrm{ppm})\end{array}\end{array}$ 
cjlh149a1.1.fid
$1 \mathrm{H} 300.1 \mathrm{MHz}$ Job 70076 Lamb Claire J $149 \mathrm{~A} 1 \mathrm{CDCl} 3 \quad 24.9 \diamond \mathrm{C}$

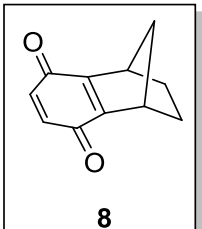

8

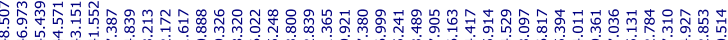

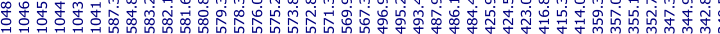

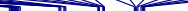

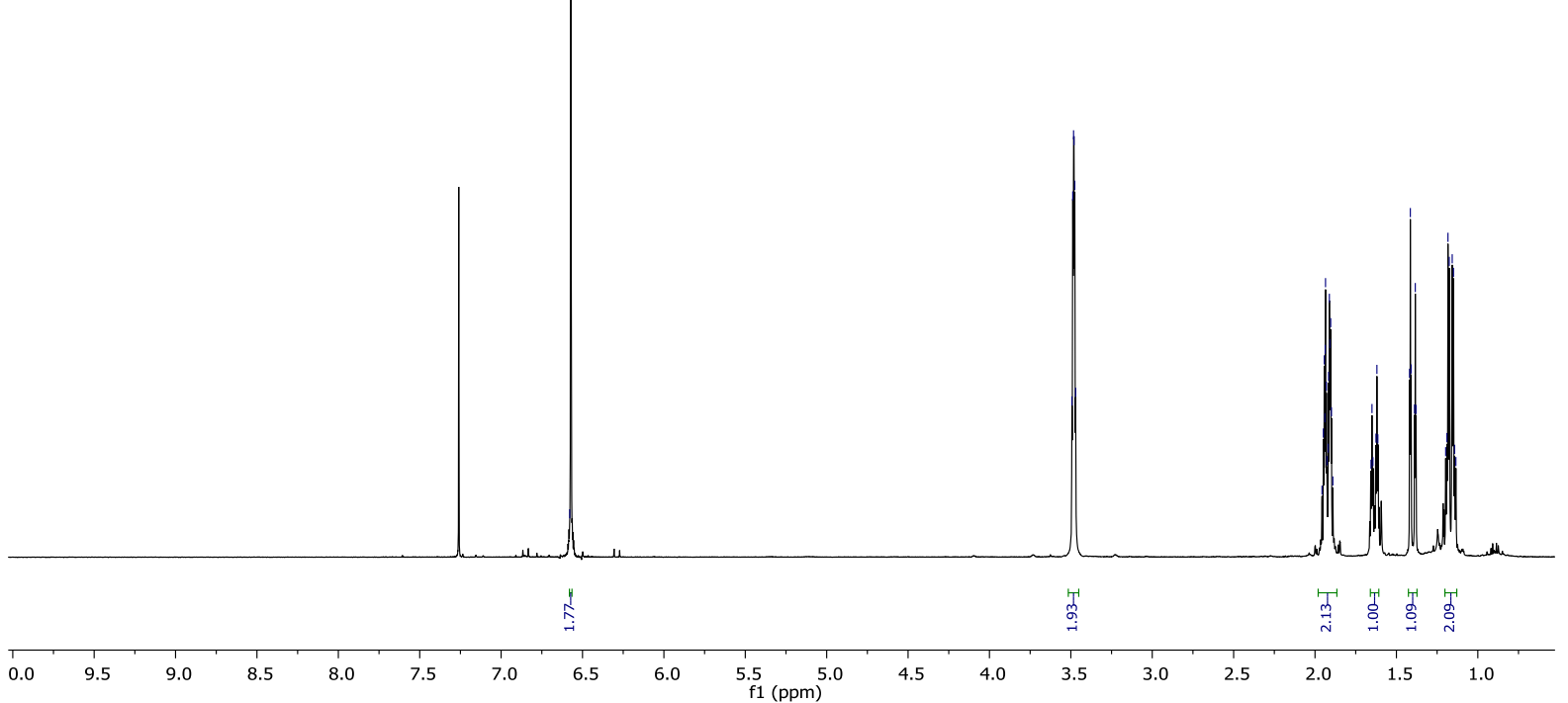

cjlc141a1.1.fid

13C 100.6MHz Job 30652 Lamb Claire J $141 \mathrm{~A} 1 \mathrm{CDCl} 3 \quad 25.5 \gg \mathrm{C} \quad$ o hour $29 \mathrm{~min}$
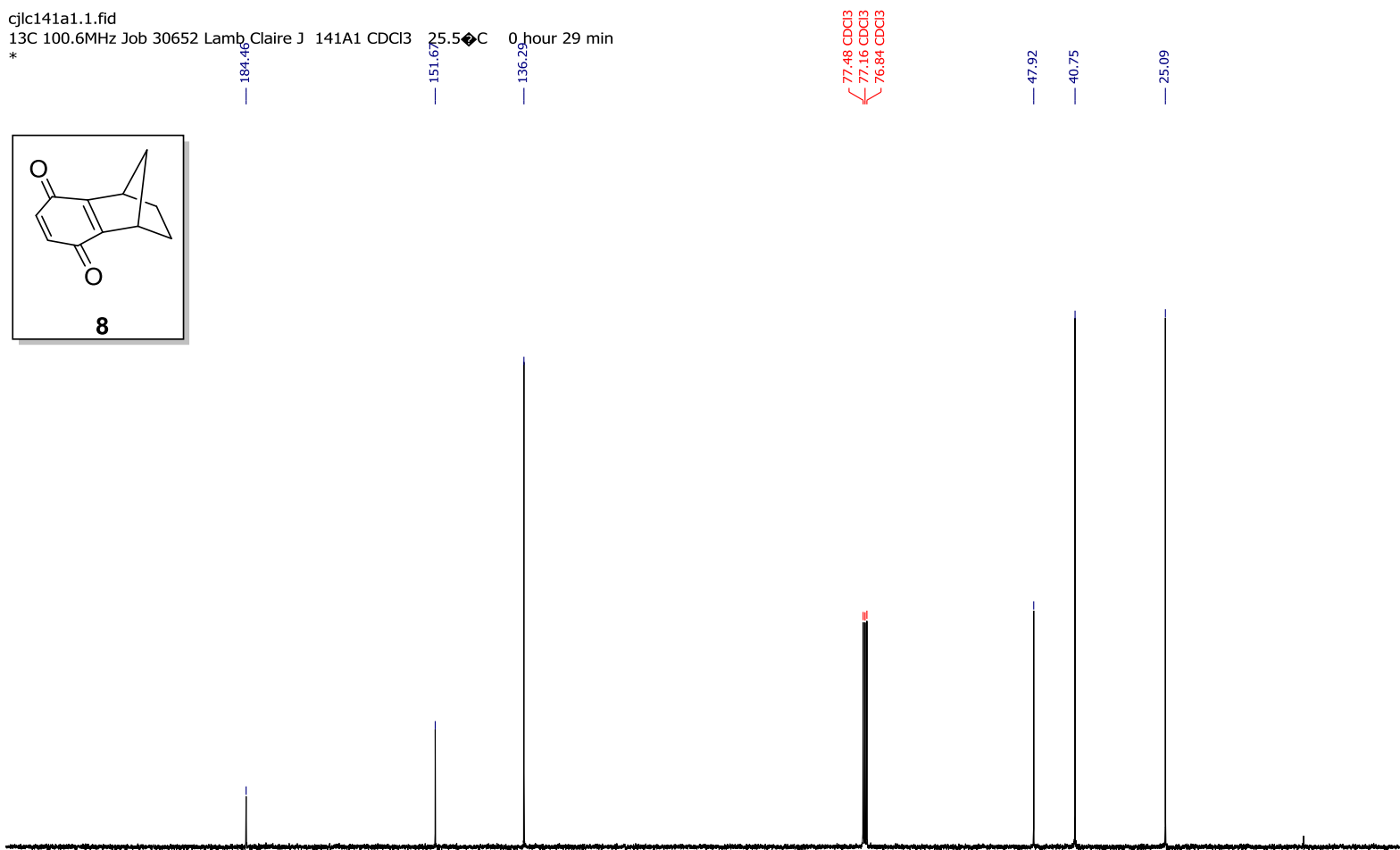

$\begin{array}{llllllllllllllllllllllllllll}220 & 210 & 200 & 190 & 180 & 170 & 160 & 150 & 140 & 130 & 120 & 110 & 100 & 90 & 80 & 70 & 60 & 50 & 40 & 30 & 20 & 10 & 0 & -10\end{array}$ 
cjlh151c1.1.fid

1H 300.1MHz Job 72522 Lamb Claire J 151C1 CDCl3

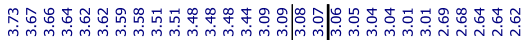
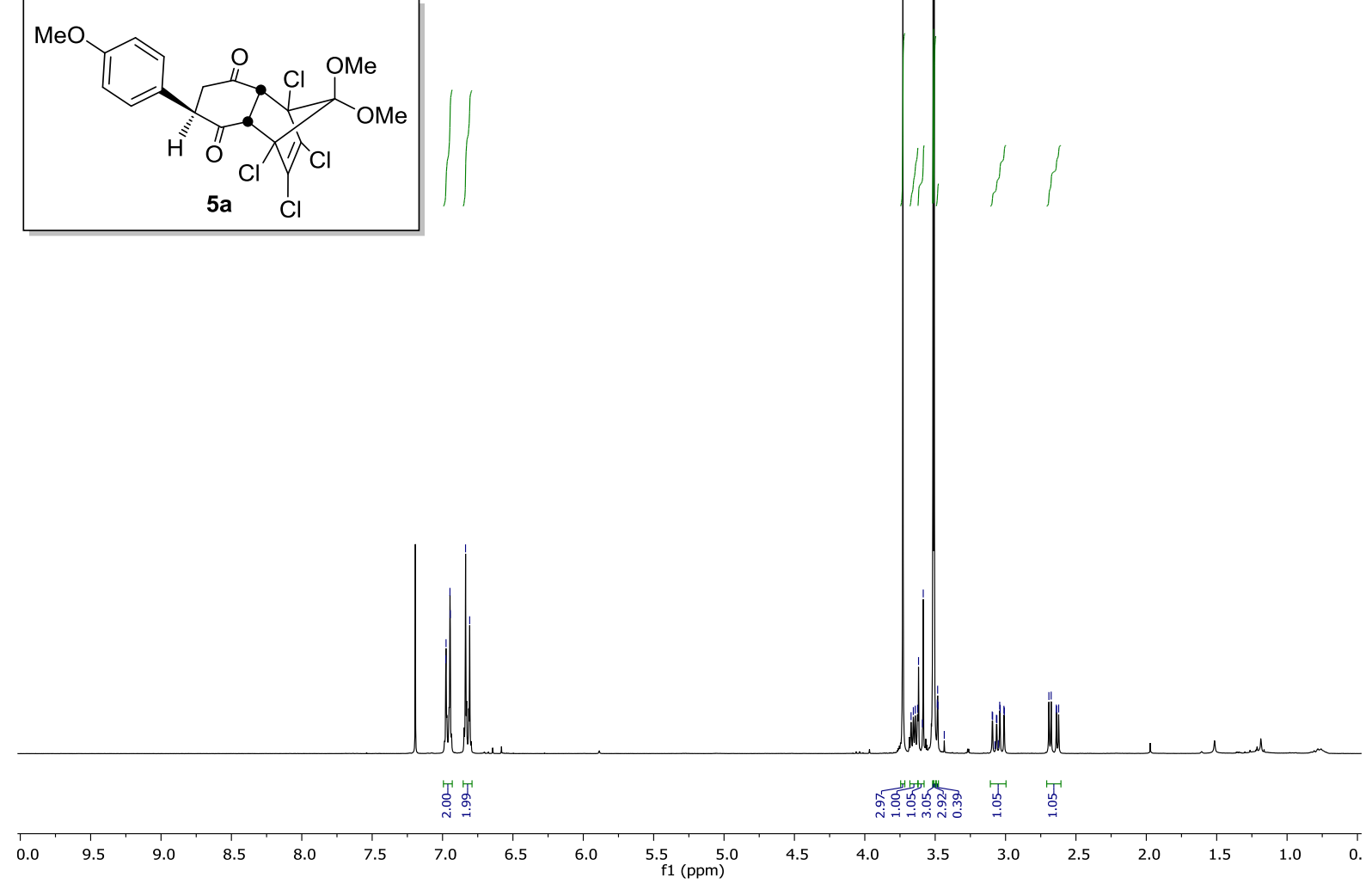

cjlc183a.1.fid

13C 100.6MHz Jgb 31899 Lamb Claire J $183 \mathrm{~A}$ CDC్ $3325.1 \diamond \mathrm{C} \quad 1$ hour 12 min

กิ่

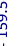

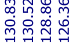

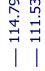

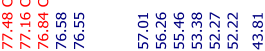

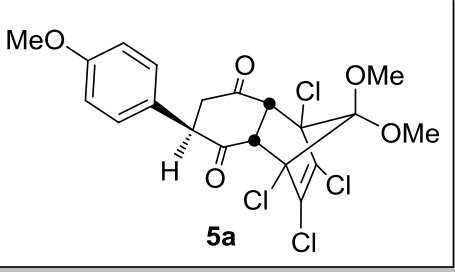

-

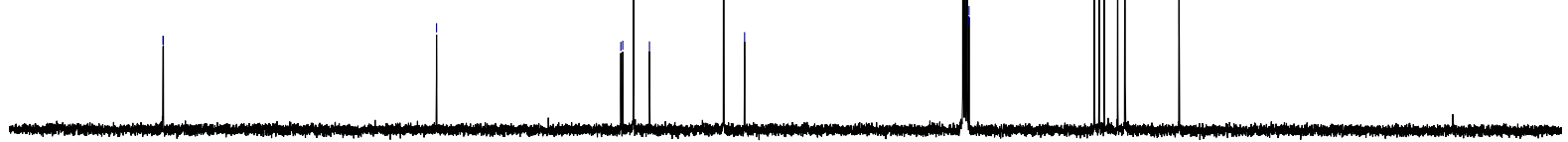

$\begin{array}{llllllllllllllllllllllllllll}220 & 210 & 200 & 190 & 180 & 170 & 160 & 150 & 140 & 130 & 120 & \begin{array}{c}110 \\ \mathrm{f} 1(\mathrm{ppm})\end{array} & 100 & 80 & 70 & 60 & 50 & 40 & 30 & 20 & 10 & 0 & -10\end{array}$ 


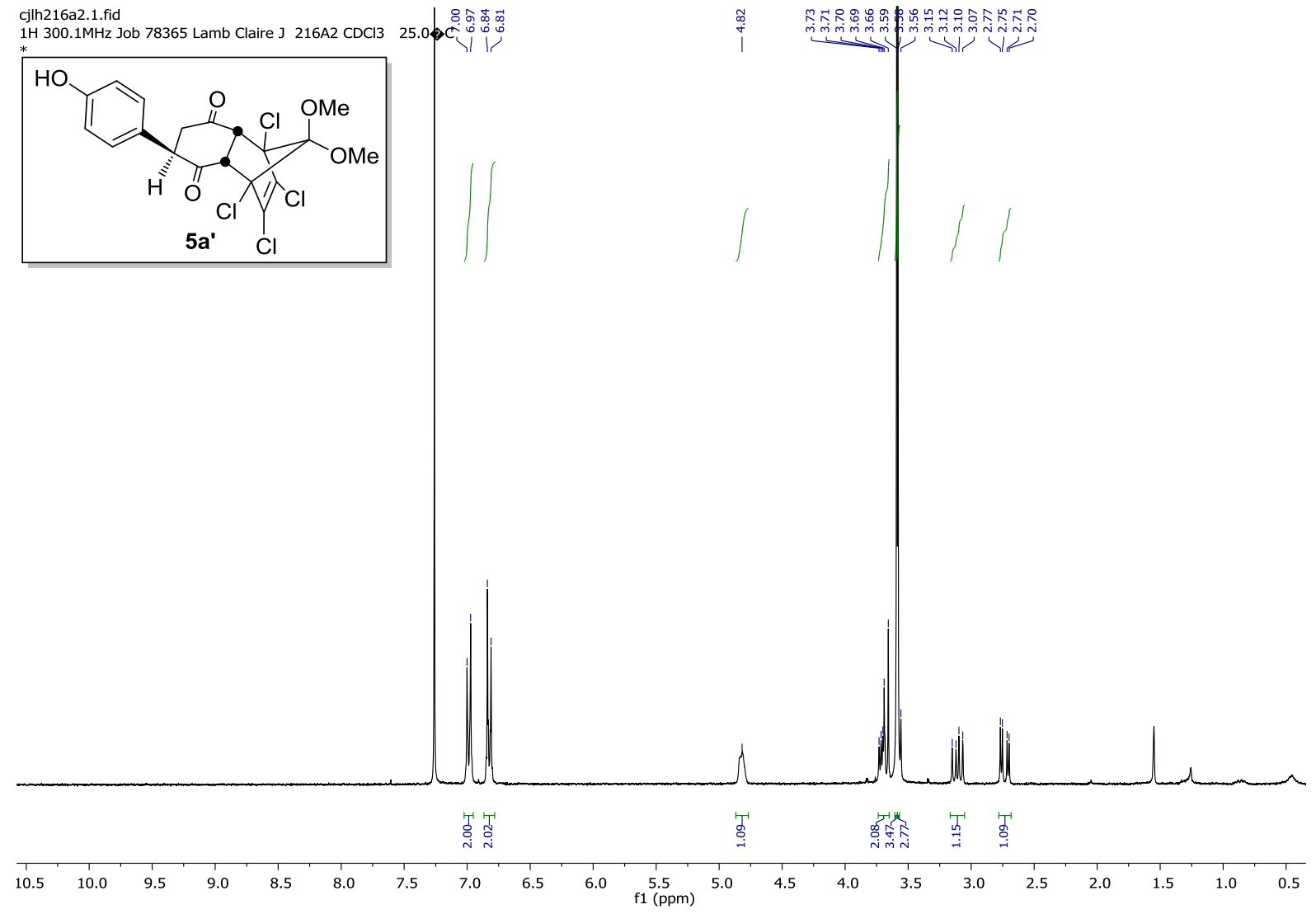

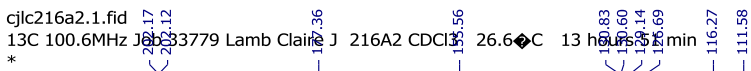

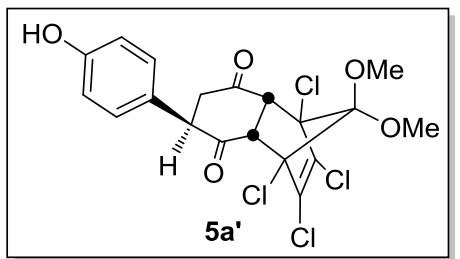

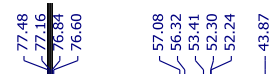

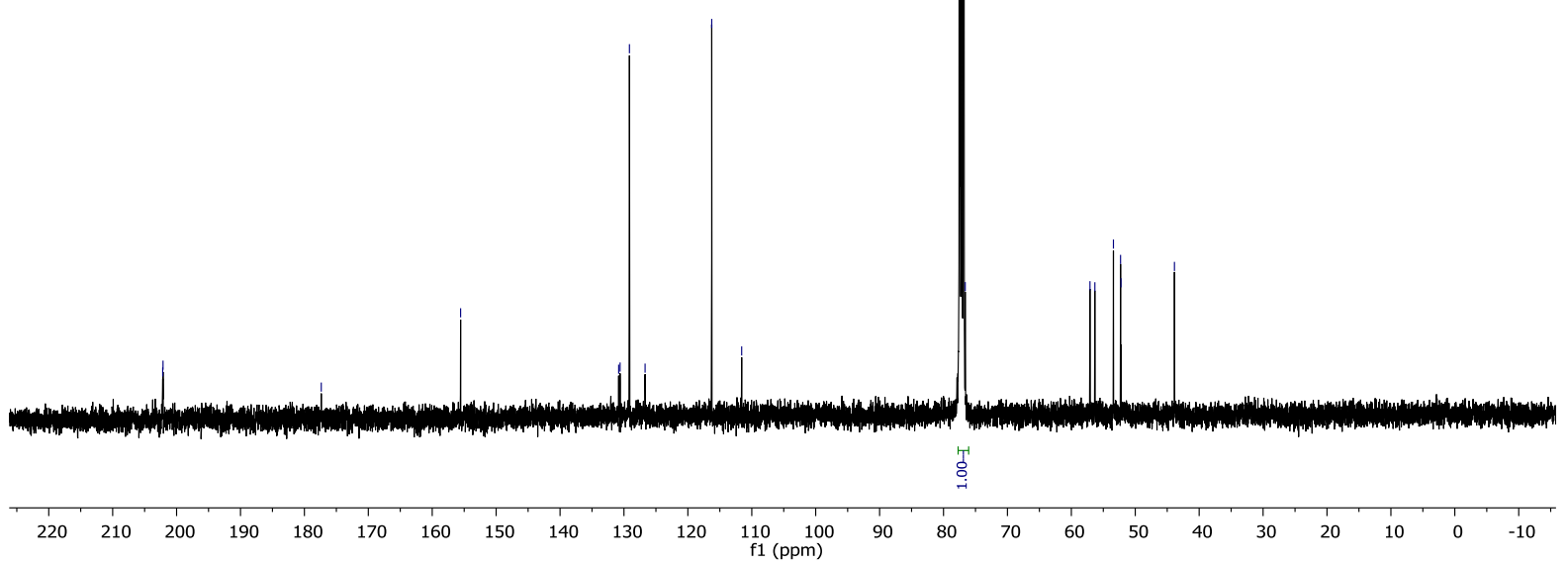



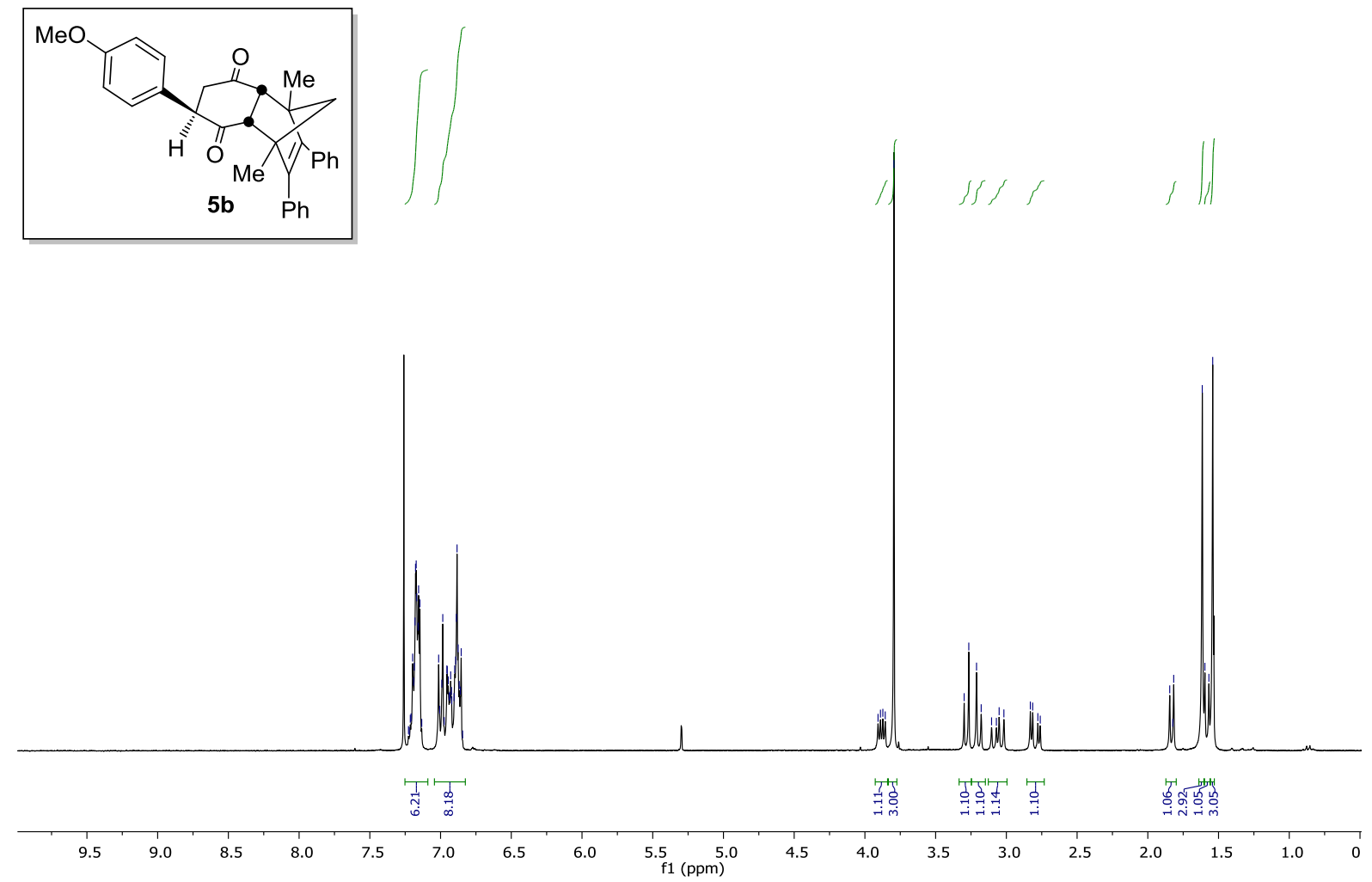

cjlc218a1a.1.fid

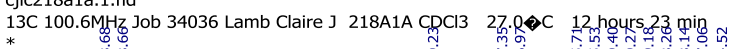

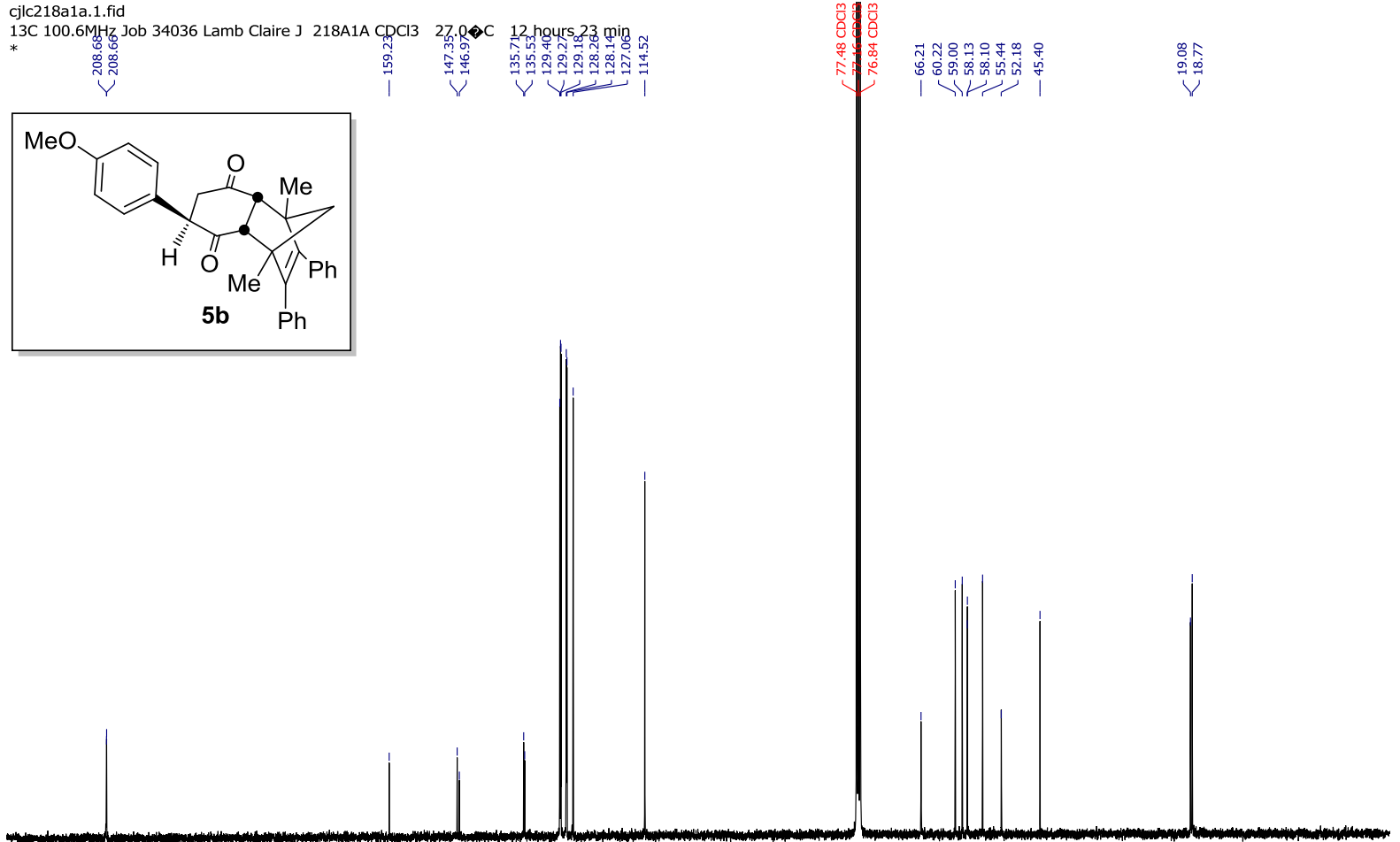

$\begin{array}{llllllllllll}220 & 210 & 200 & 190 & 180 & 170 & 160 & 150 & 140 & 130 & 120 & \begin{array}{c}110 \\ \mathrm{f} 1(\mathrm{ppm})\end{array}\end{array}$ 

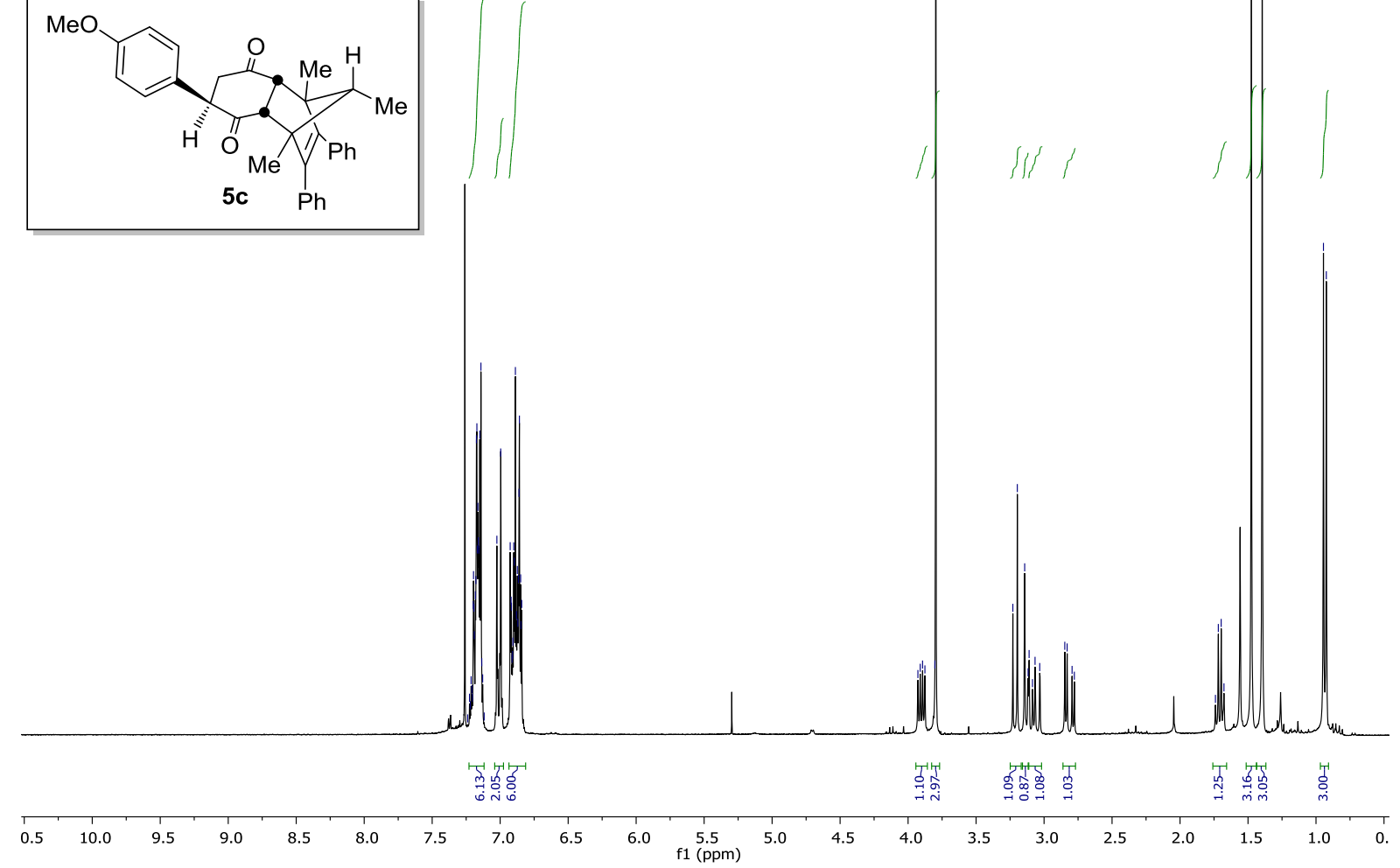

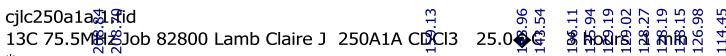

$\checkmark \sqrt{1}$

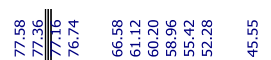
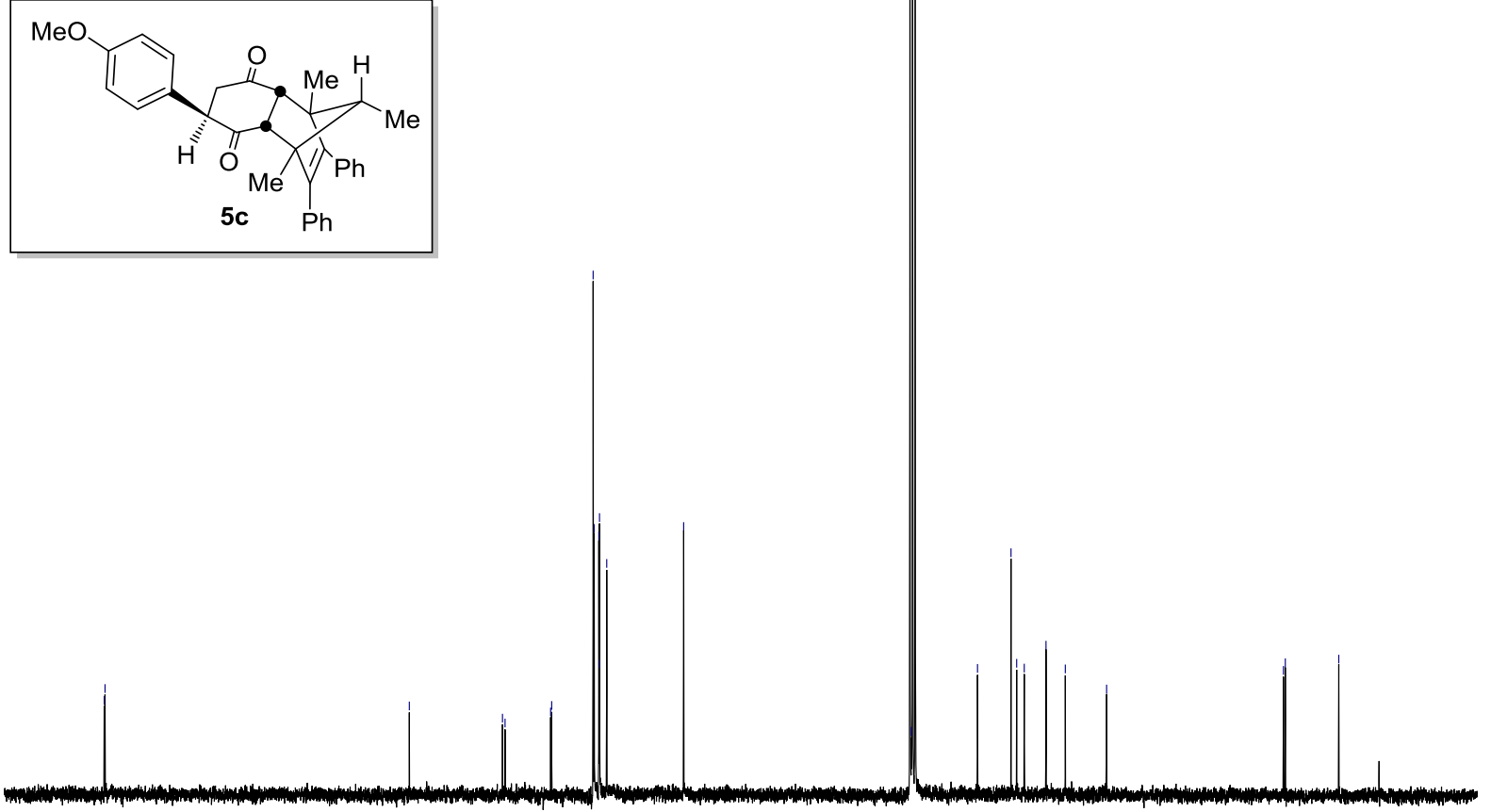

$\begin{array}{llllllllllll}220 & 210 & 200 & 190 & 180 & 170 & 160 & 150 & 140 & 130 & 120 & \begin{array}{l}110 \\ \mathrm{f} 1(\mathrm{ppm})\end{array}\end{array}$ 


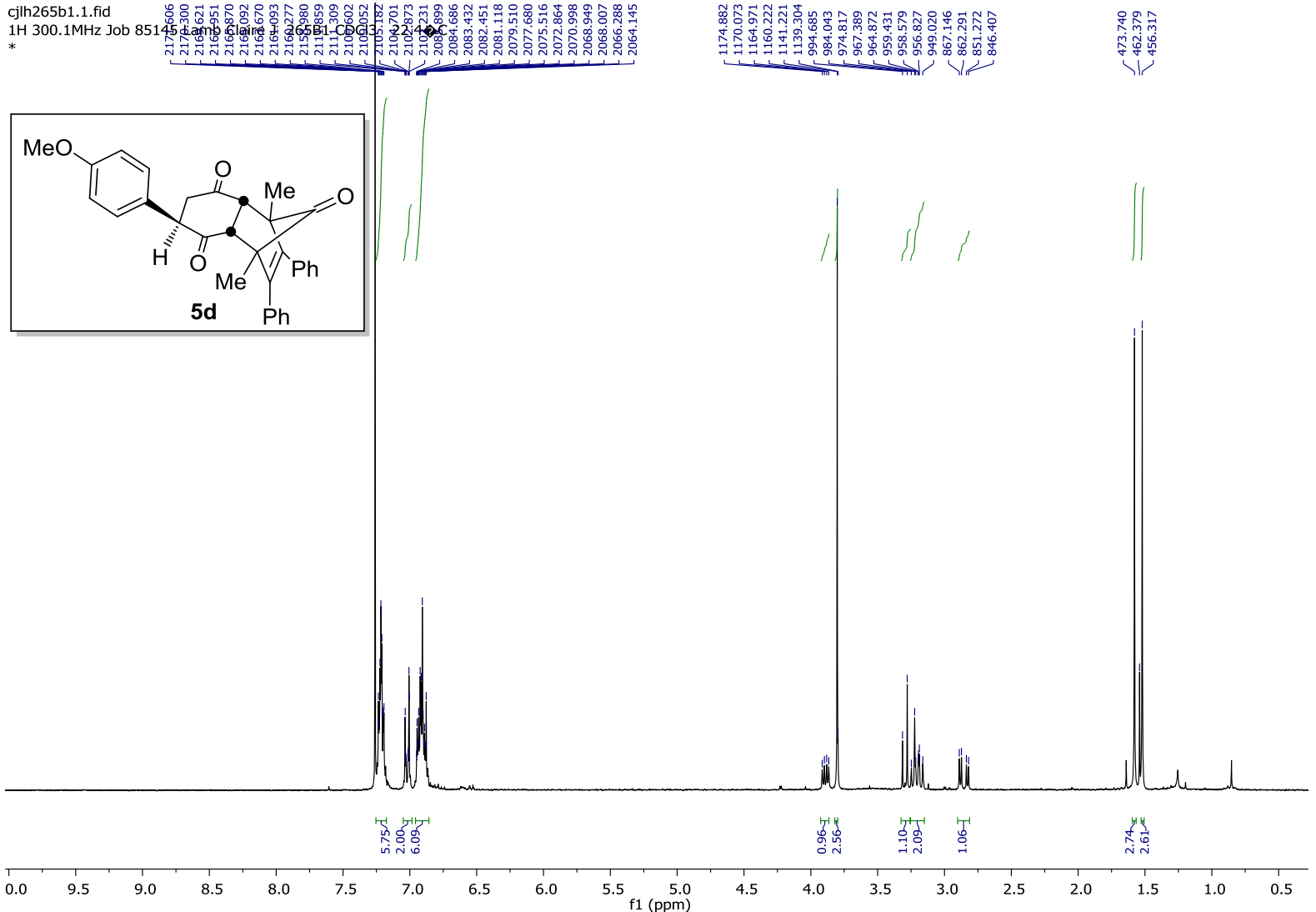

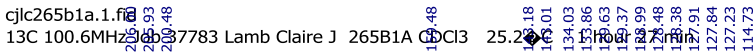

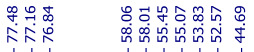

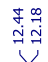
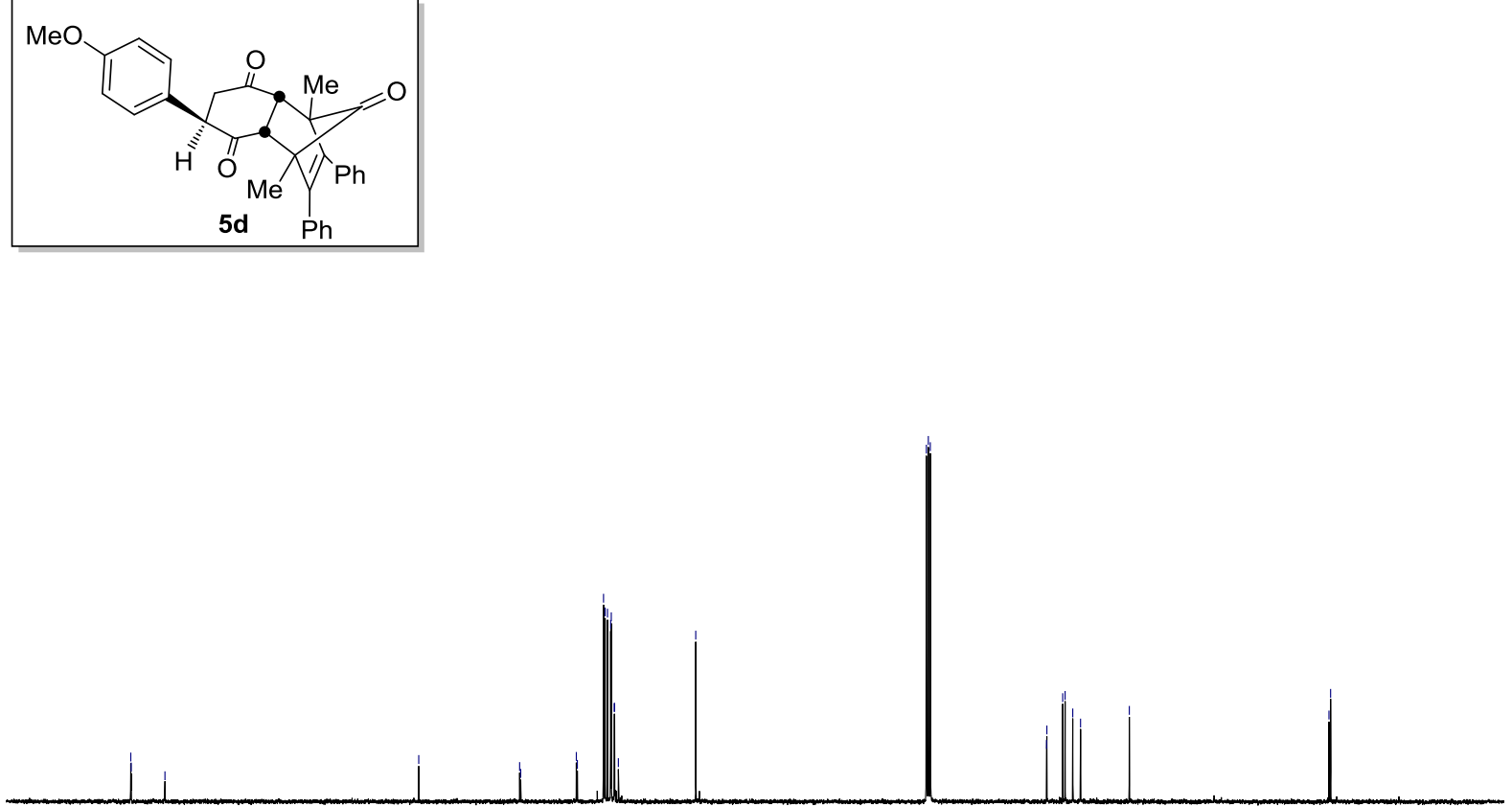

$\begin{array}{lllllllllllllllllllllllllllll}220 & 210 & 200 & 190 & 180 & 170 & 160 & 150 & 140 & 130 & 120 & 110 & 100 & 90 & 80 & 70 & 60 & 50 & 40 & 30 & 20 & 10 & 0 & -10\end{array}$ 


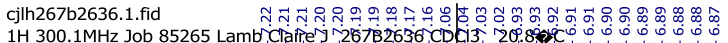

(ne

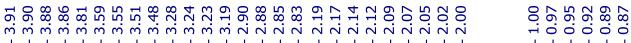
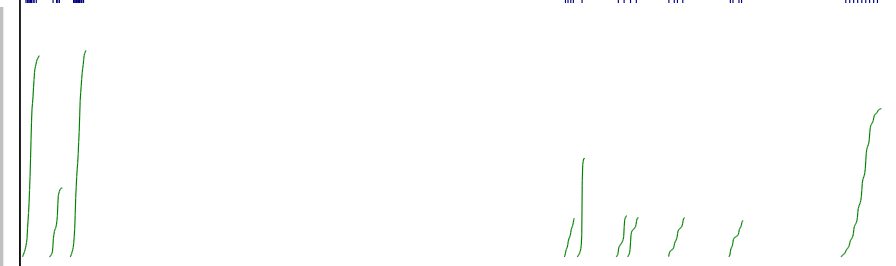

a din
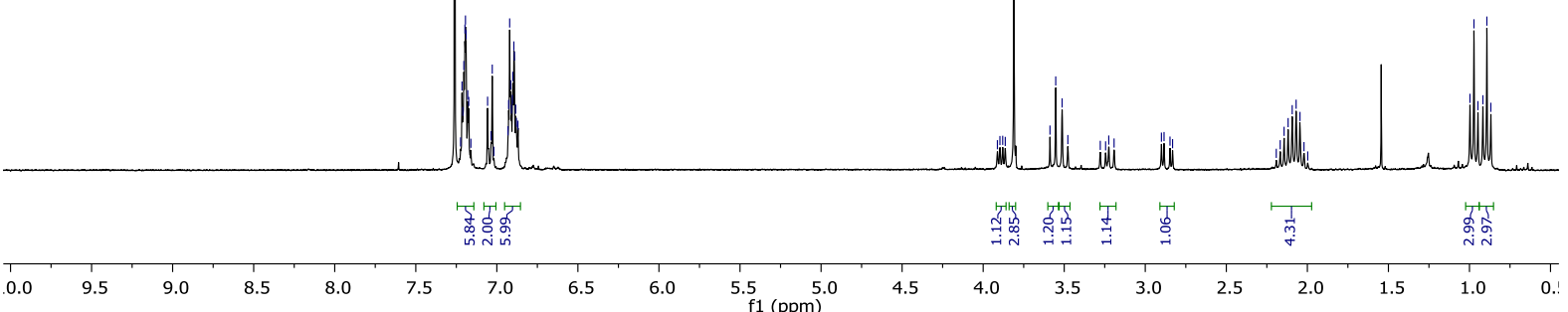

cjlc267b1.1.fid

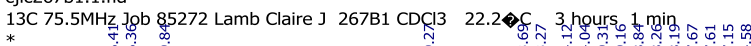

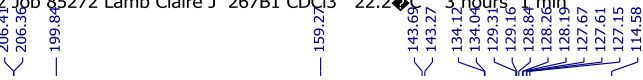
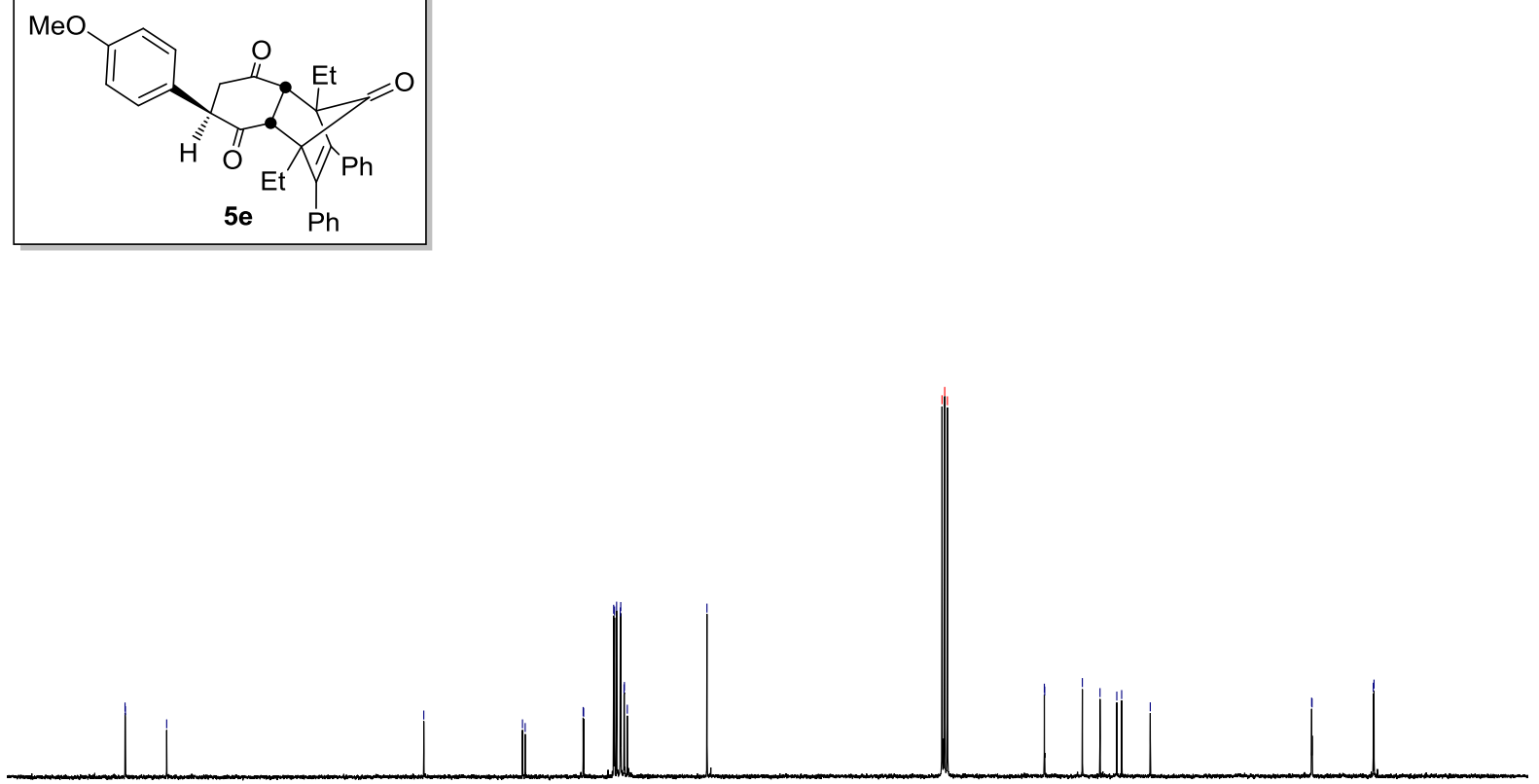

$\begin{array}{llllllllllll}220 & 210 & 200 & 190 & 180 & 170 & 160 & 150 & 140 & 130 & 120 & \begin{array}{c}110 \\ \mathrm{f} 1(\mathrm{ppm})\end{array}\end{array}$ 
cjlh271b1.1.fid

1H 300.1MHz Job 85615 Lamb Claire J 271Bricicile

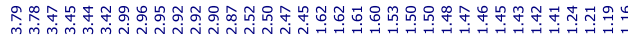
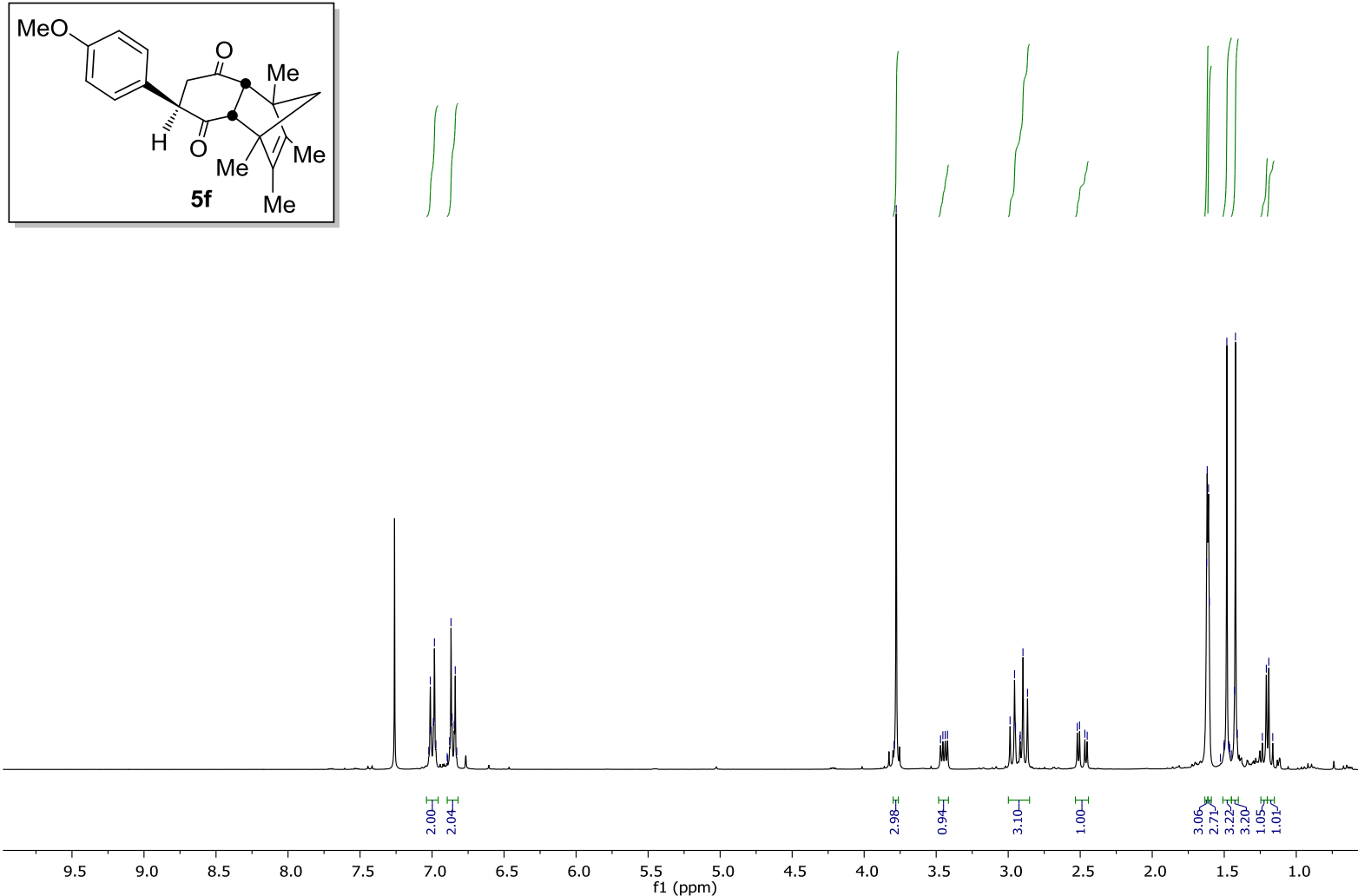

cjlc255a1c.1.fid

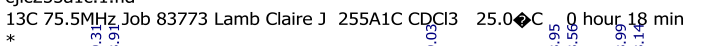
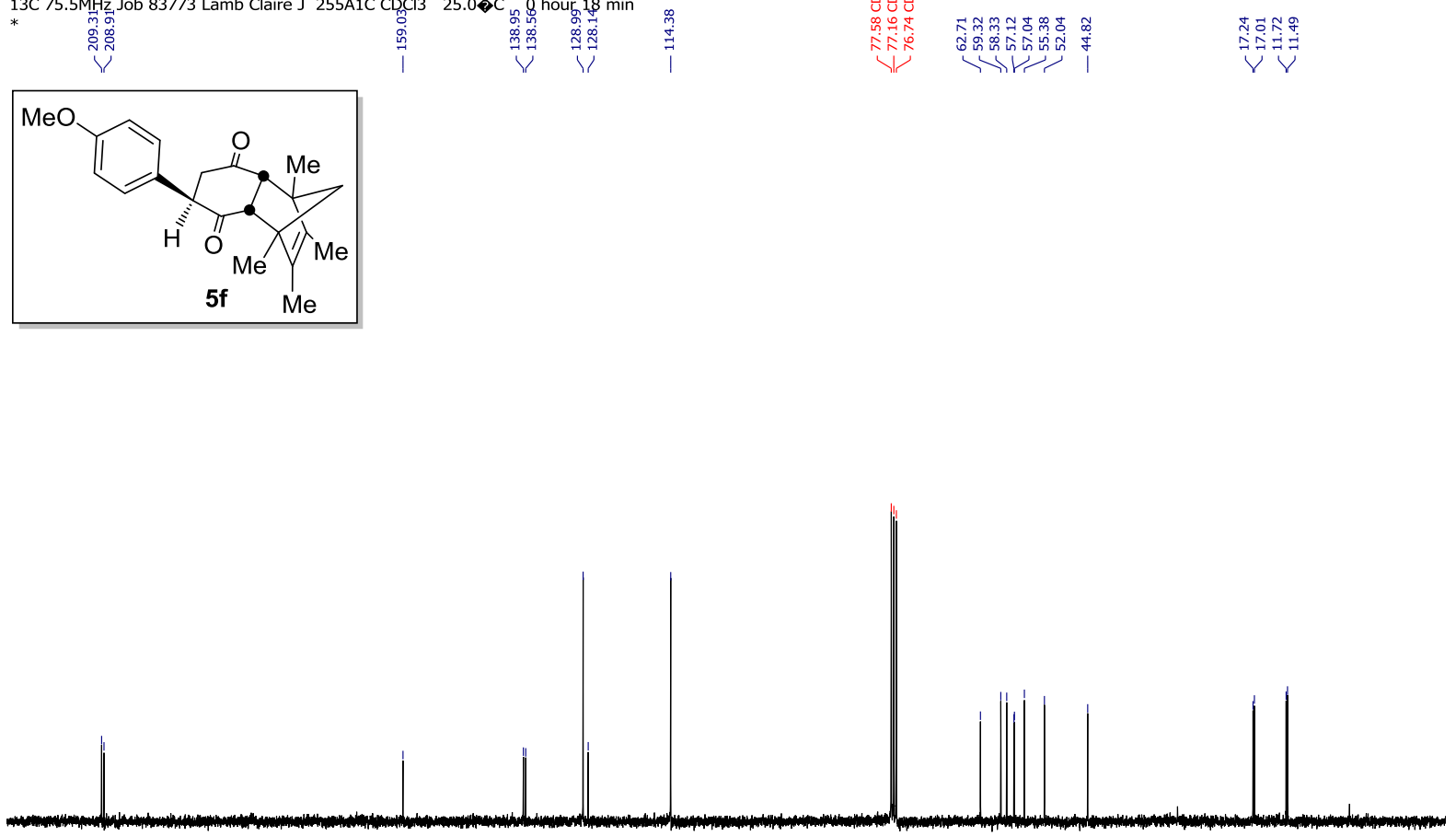

$\begin{array}{llllllllllll}220 & 210 & 200 & 190 & 180 & 170 & 160 & 150 & 140 & 130 & 120 & 110 \\ \mathrm{f} 1(\mathrm{ppm}) & 100\end{array}$ 
cjlh256b26.1.fid

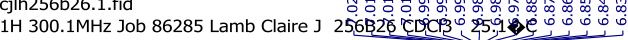
Fraction 26
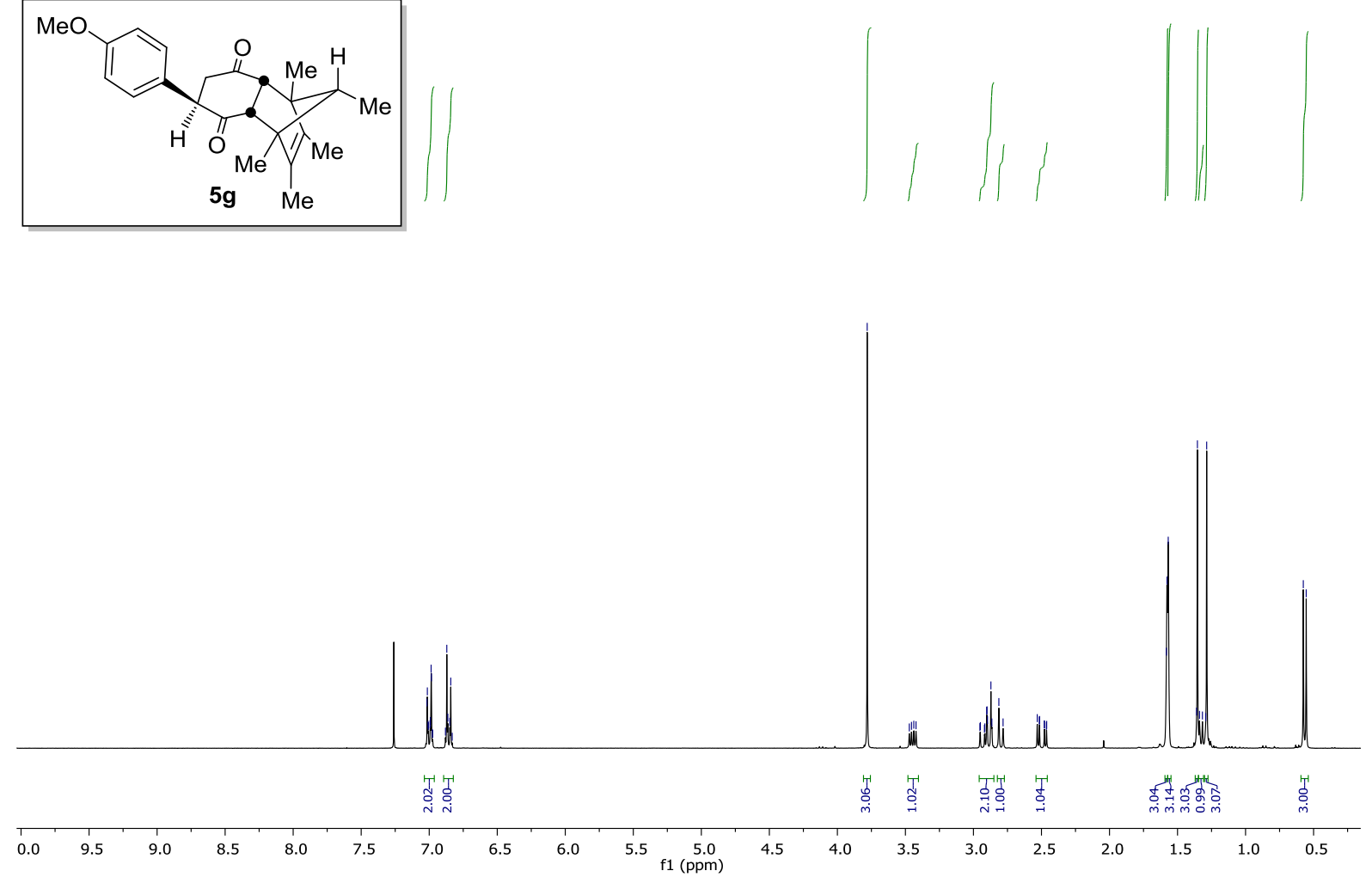

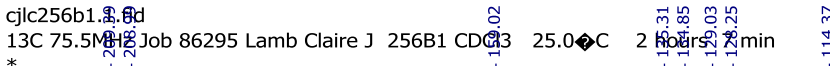

$\underset{\substack{m \\ \multirow{j}{*}{}}}{j}$

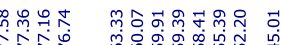

$\checkmark \mathrm{V}$

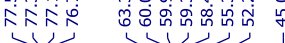

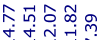
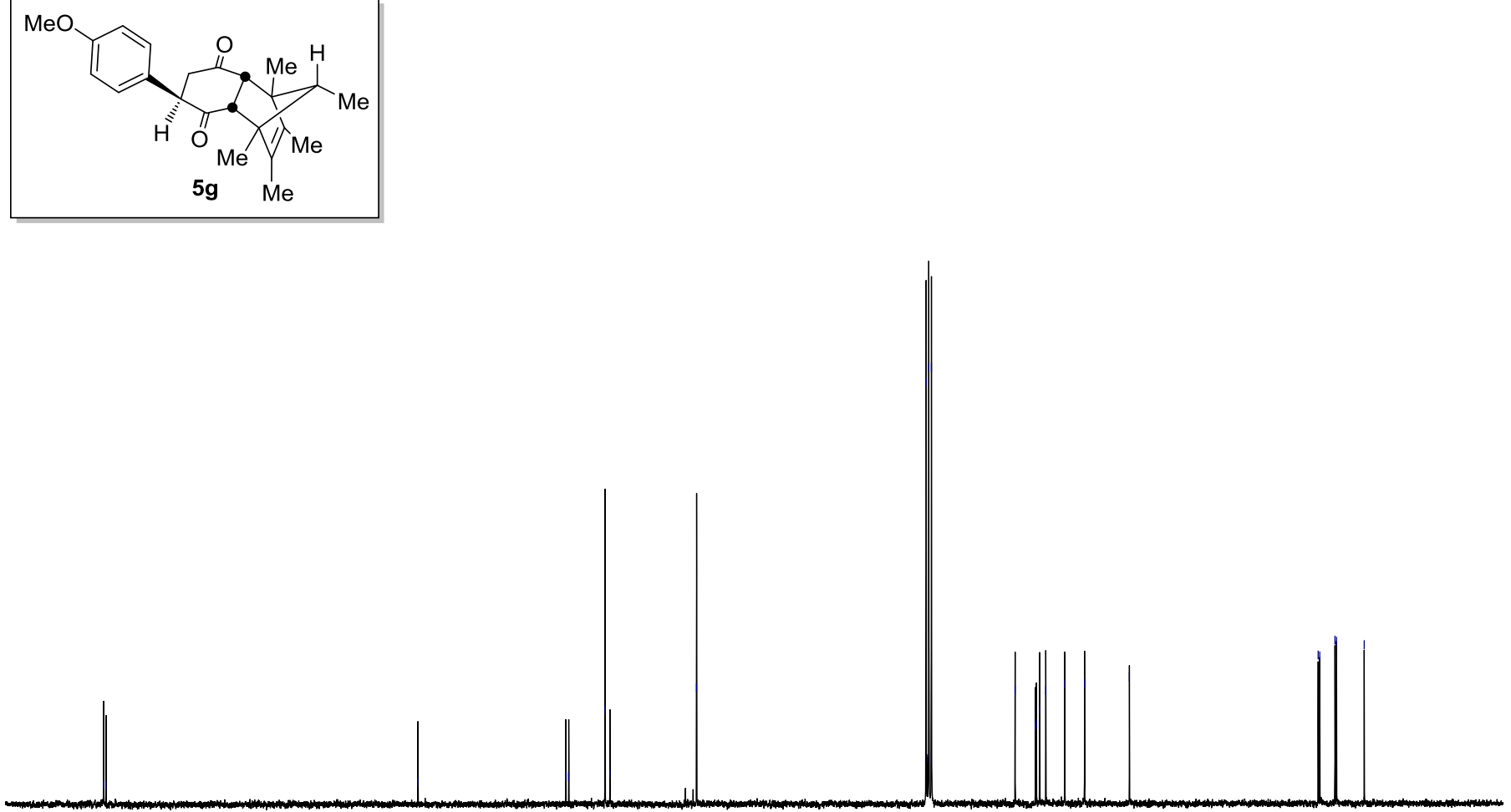

$\begin{array}{lllllllllllll}220 & 210 & 200 & 190 & 180 & 170 & 160 & 150 & 140 & 130 & 120 & 110 & 100\end{array}$ 


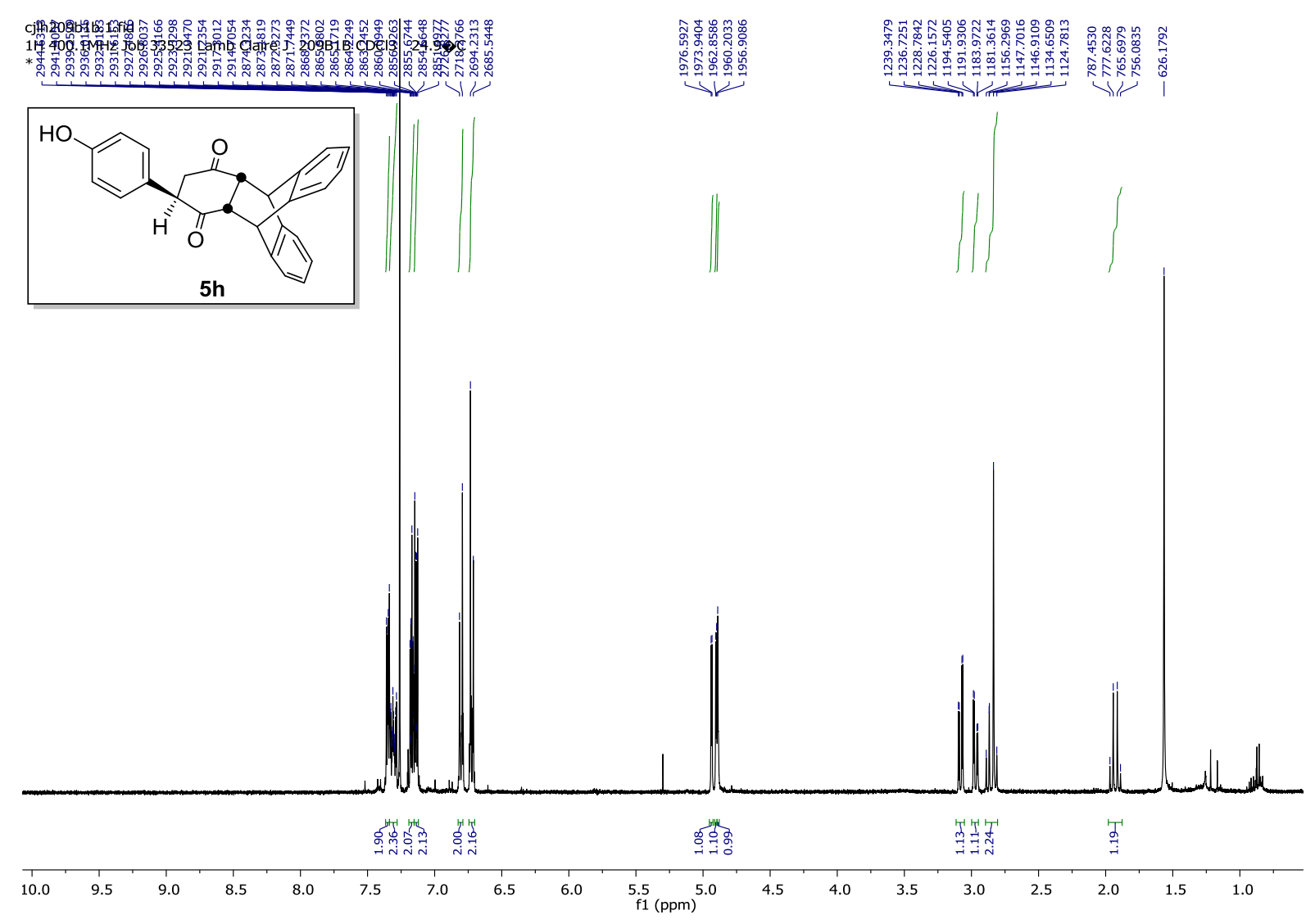

cjlc209b1b.1.fid

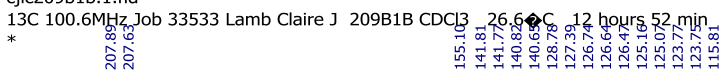
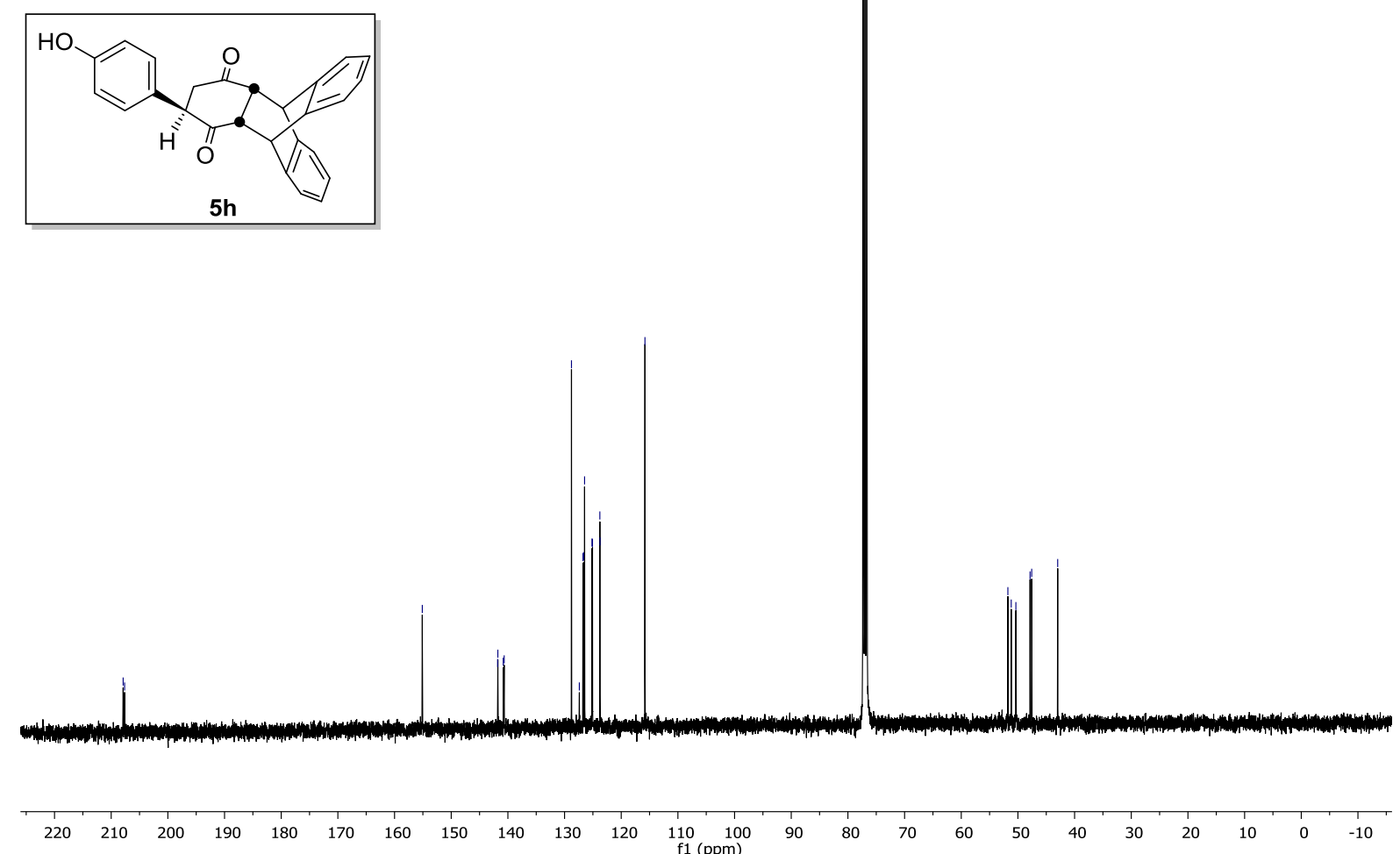


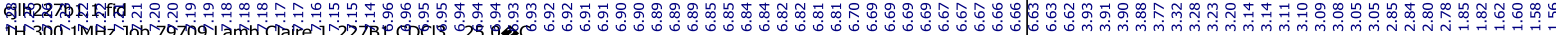

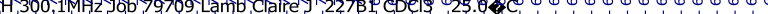
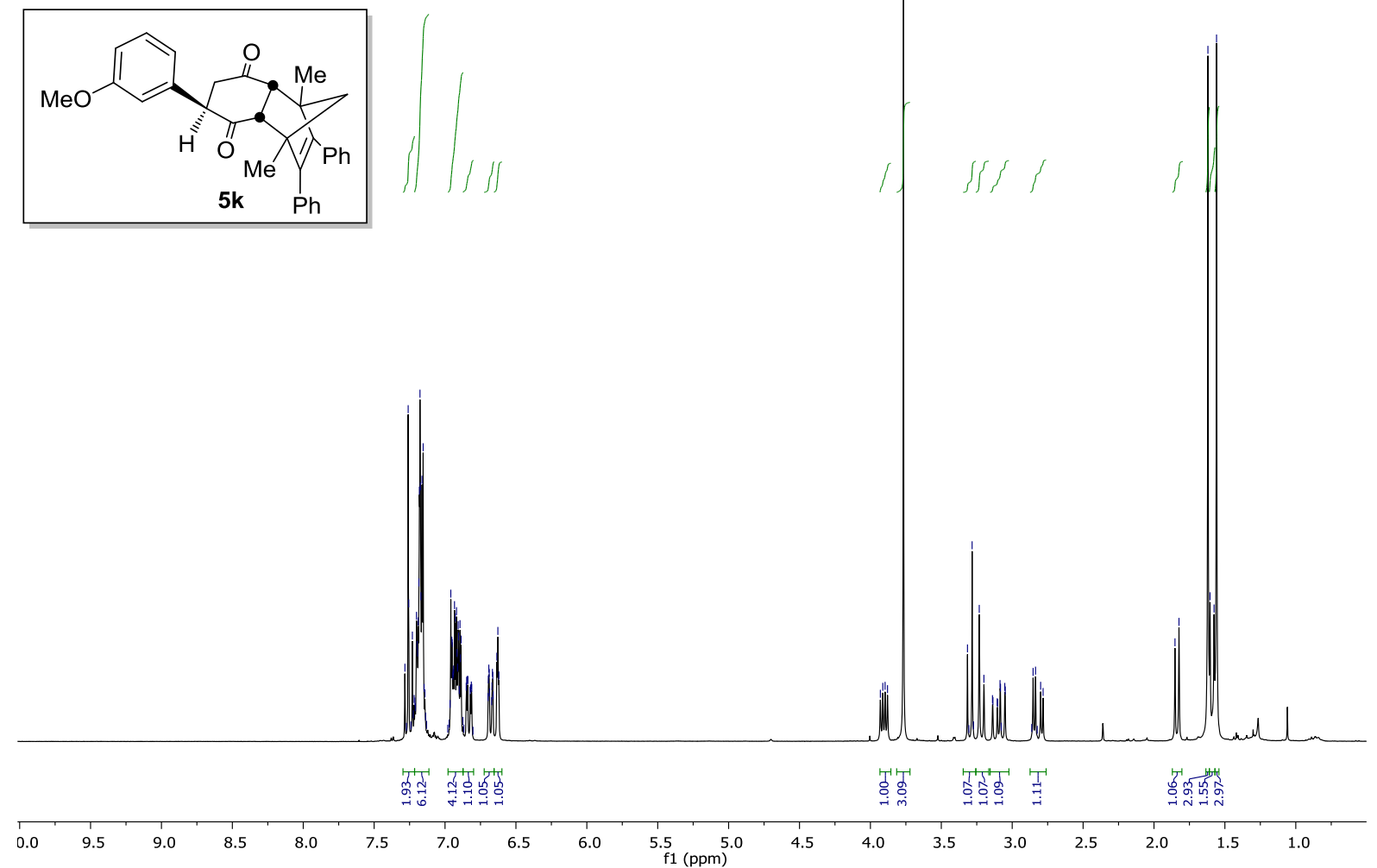

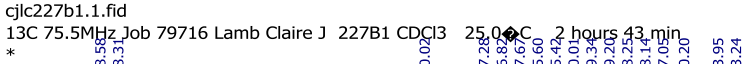

Vิ
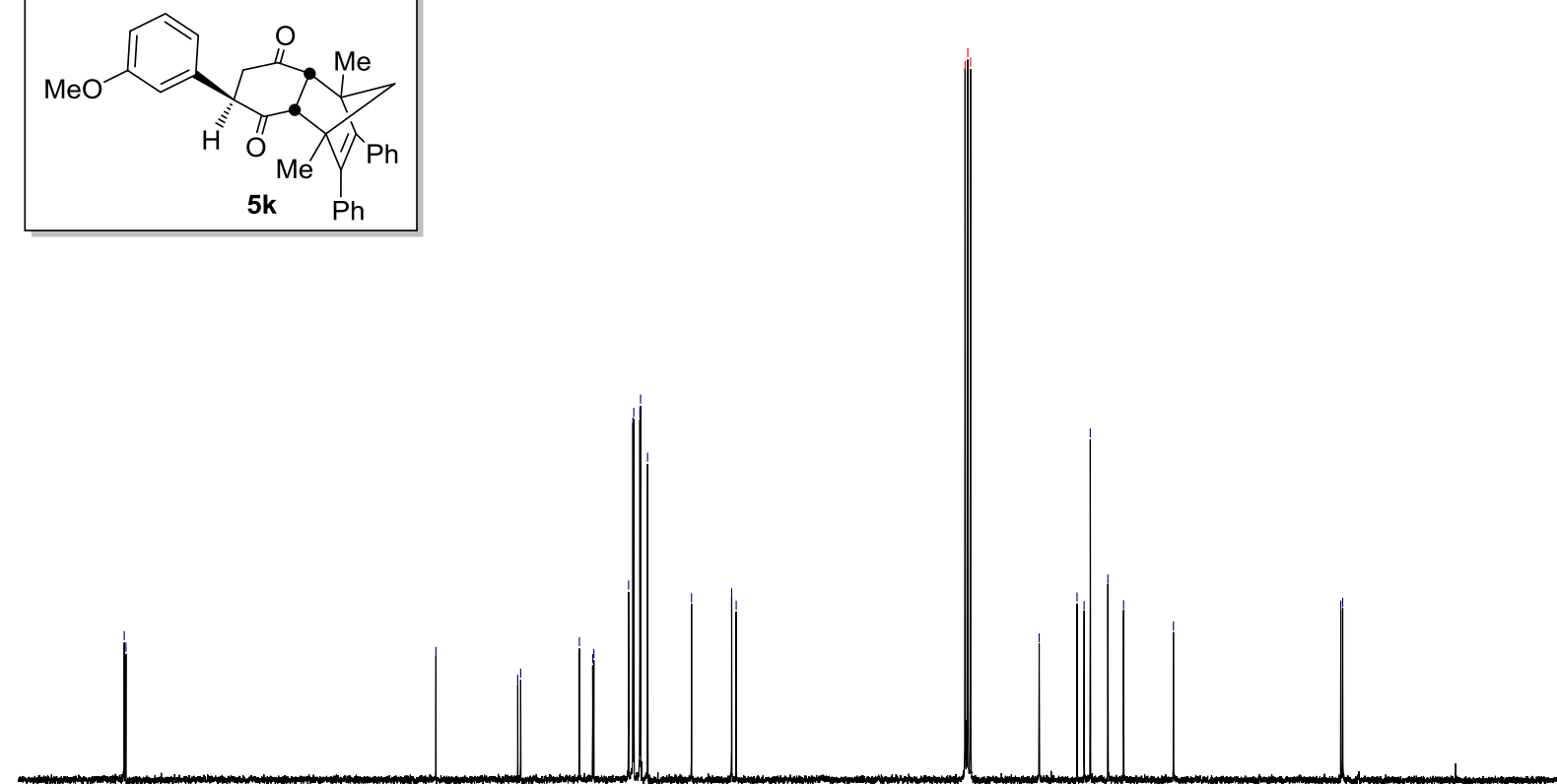

$\begin{array}{llllllllllll}220 & 210 & 200 & 190 & 180 & 170 & 160 & 150 & 140 & 130 & 120 & \begin{array}{c}110 \\ \mathrm{f} 1(\mathrm{ppm})\end{array}\end{array}$ 


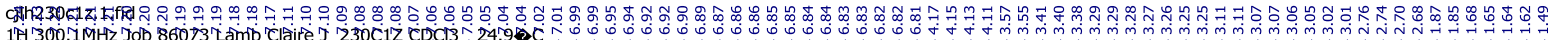

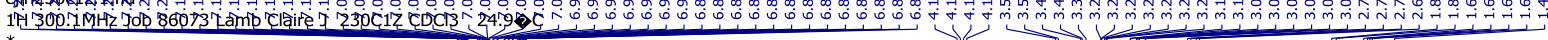

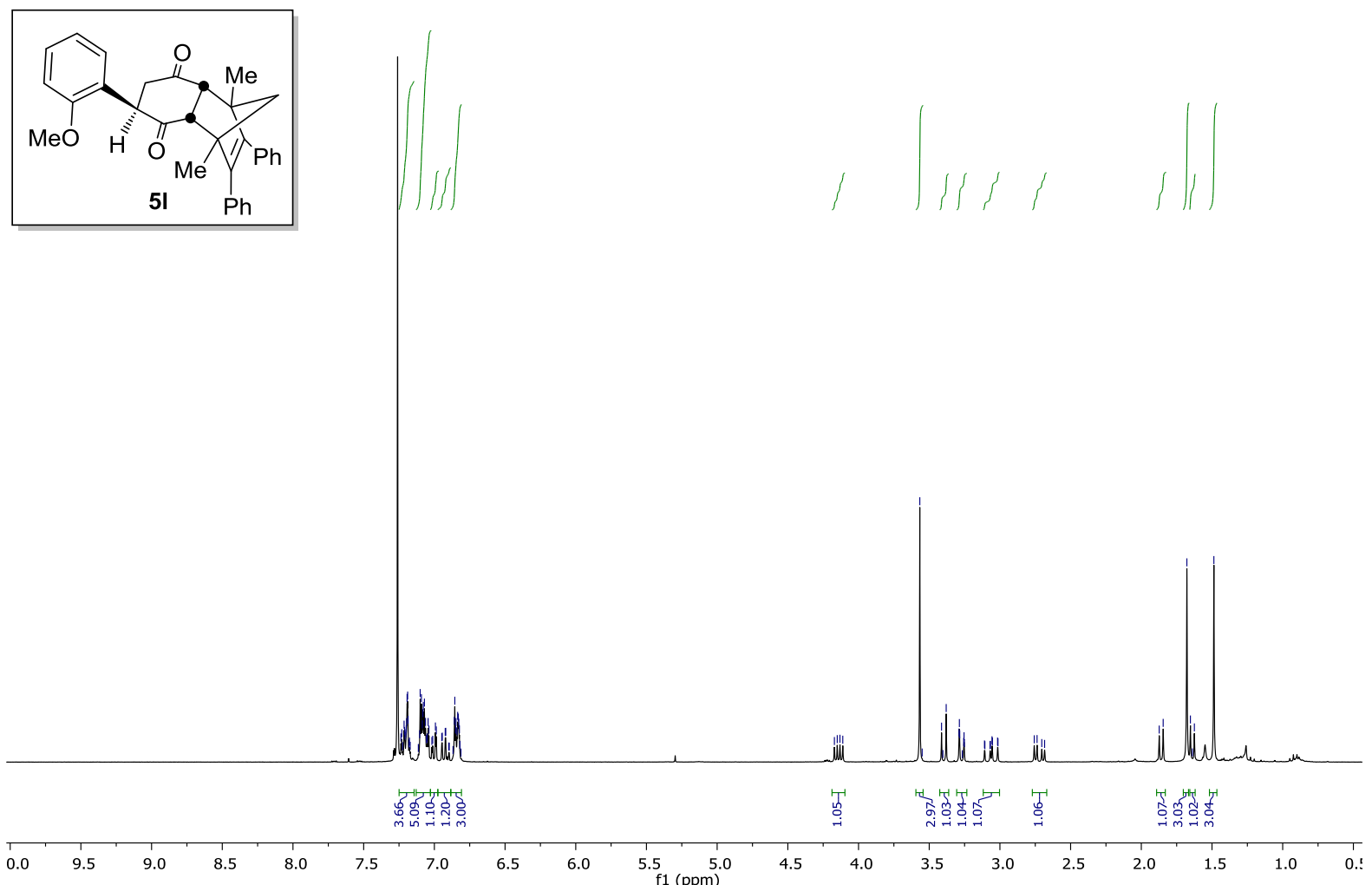

13C 75.5MHzidobob 86077 Lamb Claire J 230C1Z CDCl3

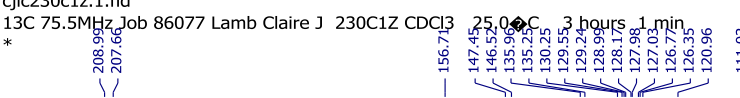

ก

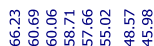

|

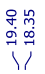
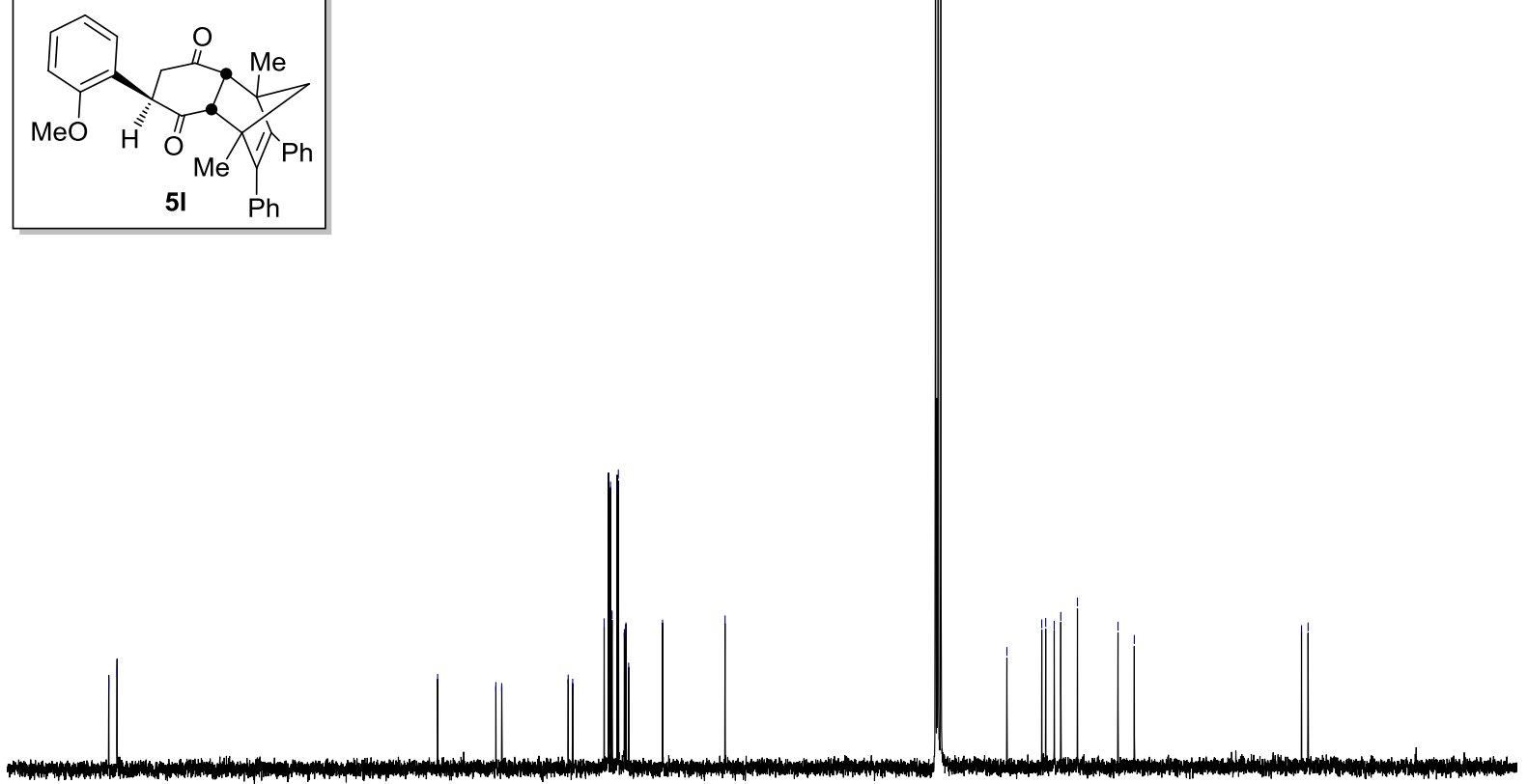

$\begin{array}{llllllllllll}220 & 210 & 200 & 190 & 180 & 170 & 160 & 150 & 140 & 130 & 120 & \underset{\mathrm{f} 1}{110}(\mathrm{ppm})\end{array}$

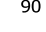

80

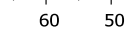

40

$\begin{array}{llll}20 & 10 & 0 & -10\end{array}$ 


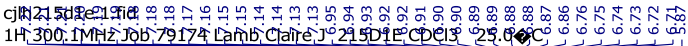

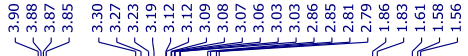
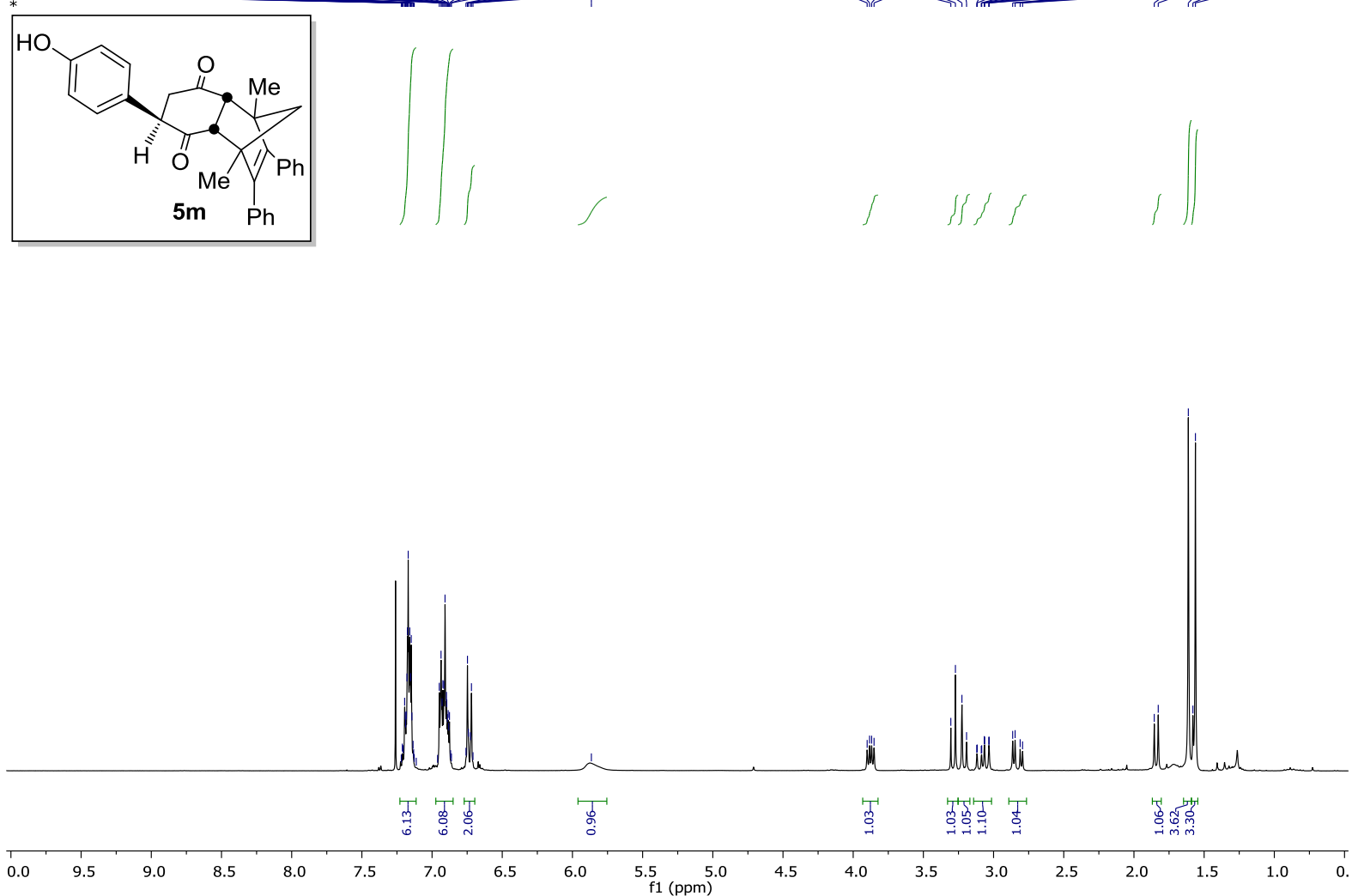

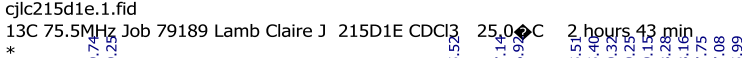

今ั0
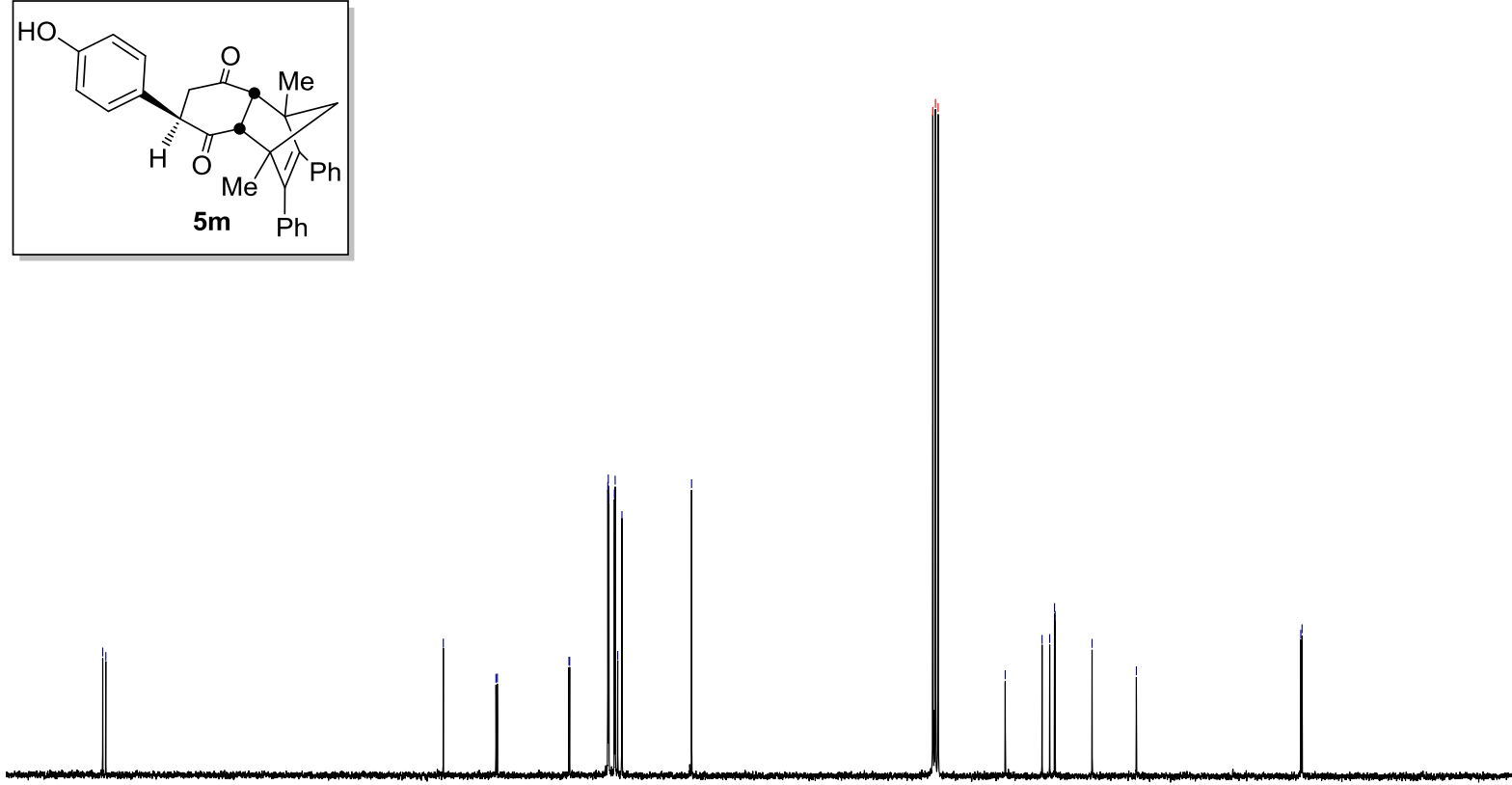

$\begin{array}{llllllllllll}220 & 210 & 200 & 190 & 180 & 170 & 160 & 150 & 140 & 130 & 120 & \begin{array}{c}110 \\ \mathrm{f} 1(\mathrm{ppm})\end{array}\end{array}$ 


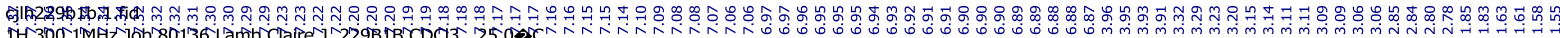

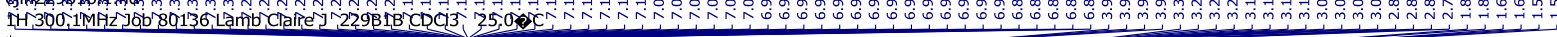
(n)
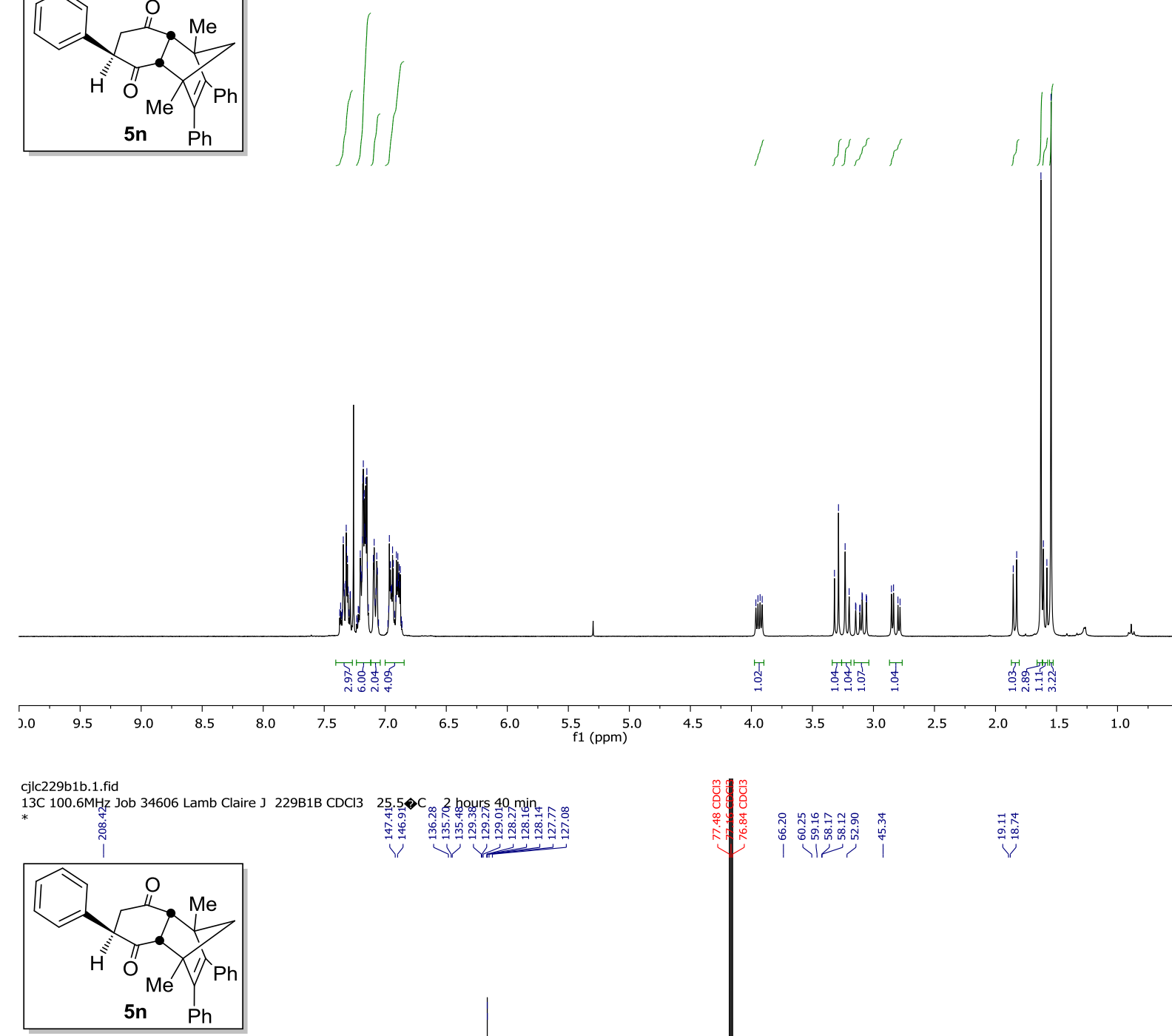

cjlc229b1b.1.fid

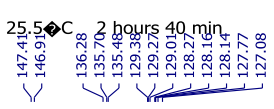

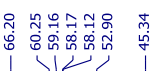

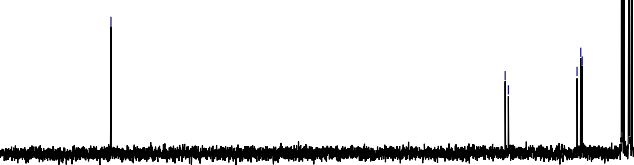

$\begin{array}{llllllllllll}220 & 210 & 200 & 190 & 180 & 170 & 160 & 150 & 140 & 130 & 120 & \begin{array}{c}110 \\ \mathrm{f} 1(\mathrm{ppm})\end{array}\end{array}$ 


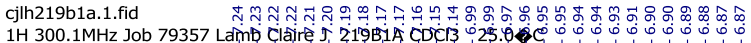

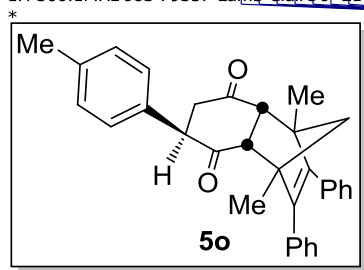

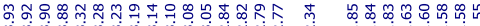

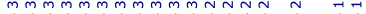
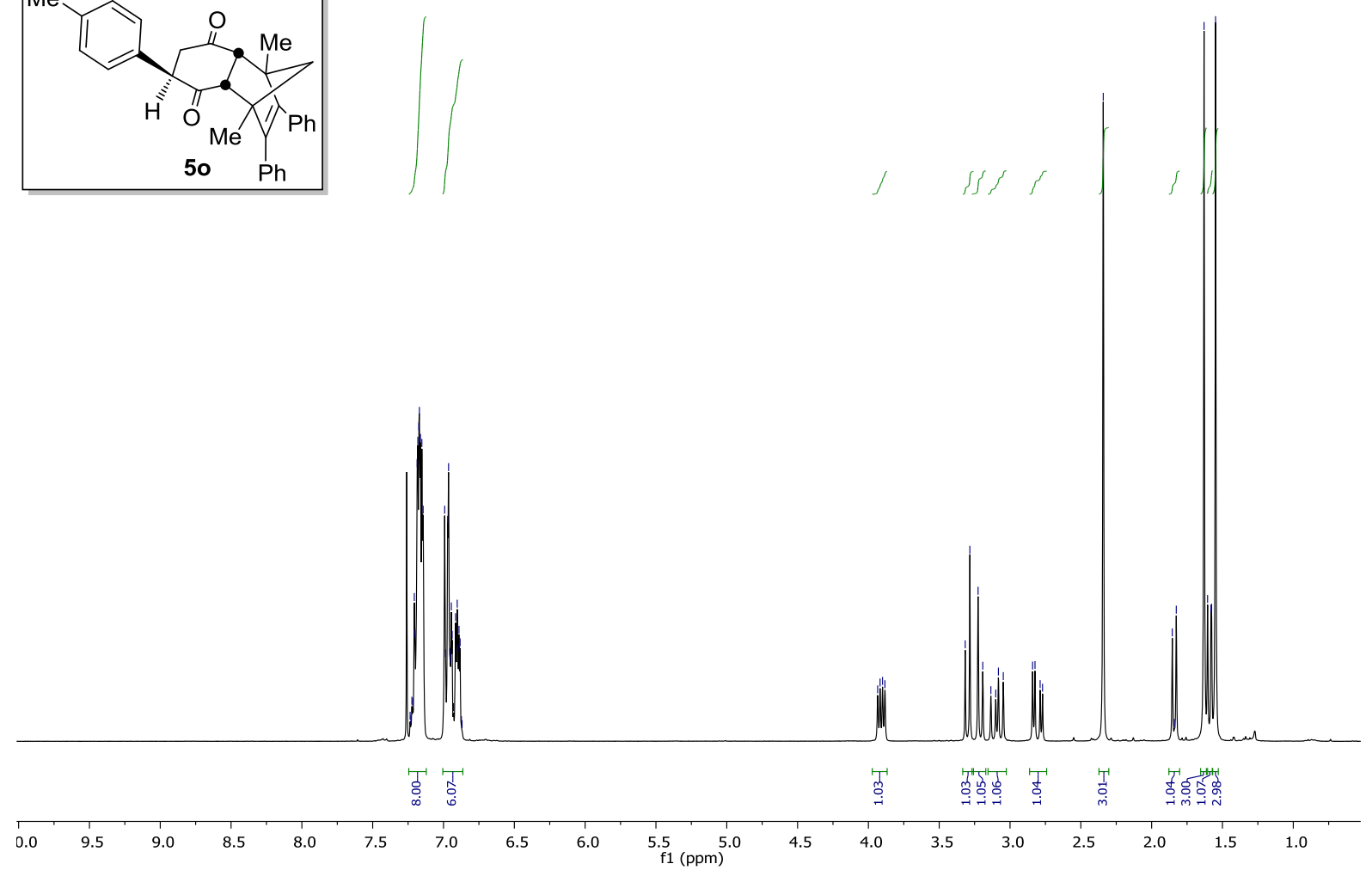

cjlc219b1a.1.fid

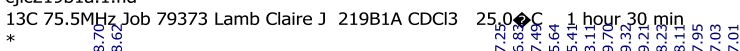

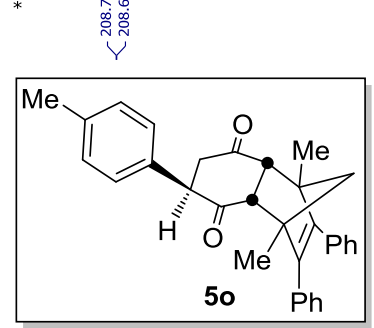

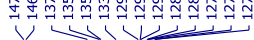




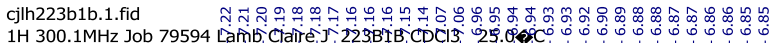

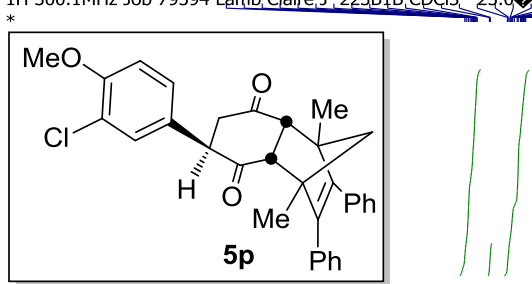

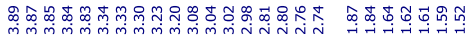

$\underbrace{1}$
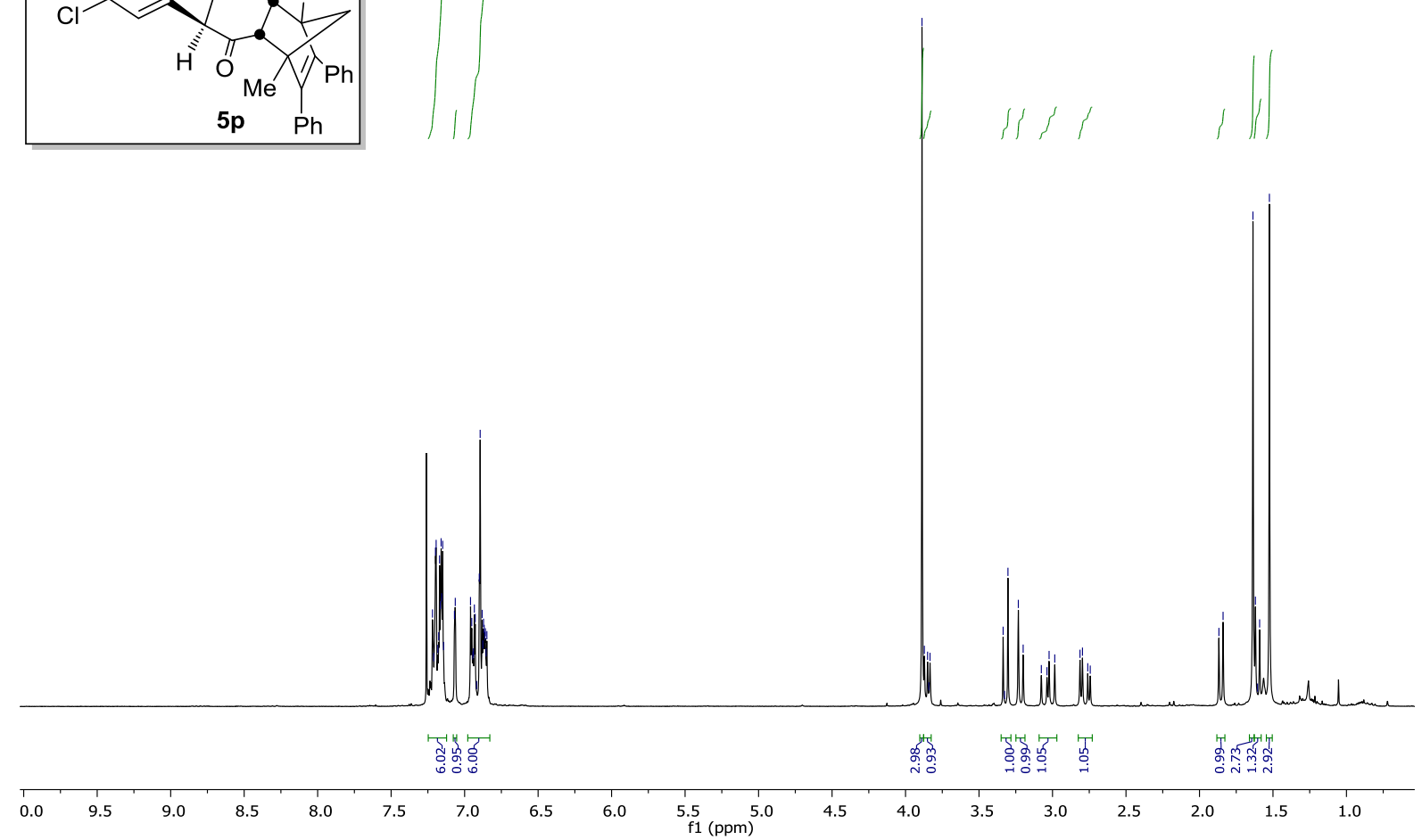

cjlc223b1b.1.fid

13C 75.5MHz Job 79595 Lamb Claire J 223B1B CDCl3 3 250 0 C 3 hours 1 min
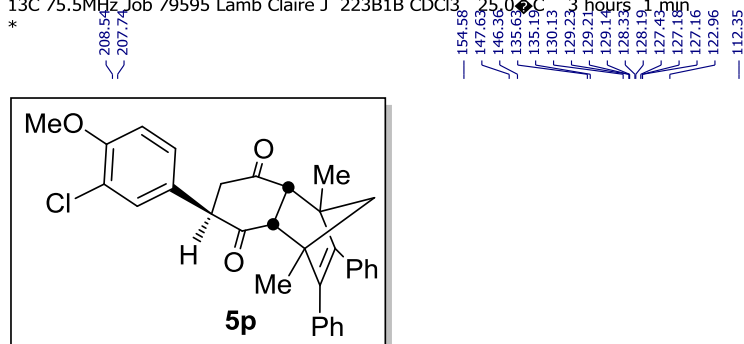

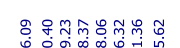

|

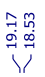

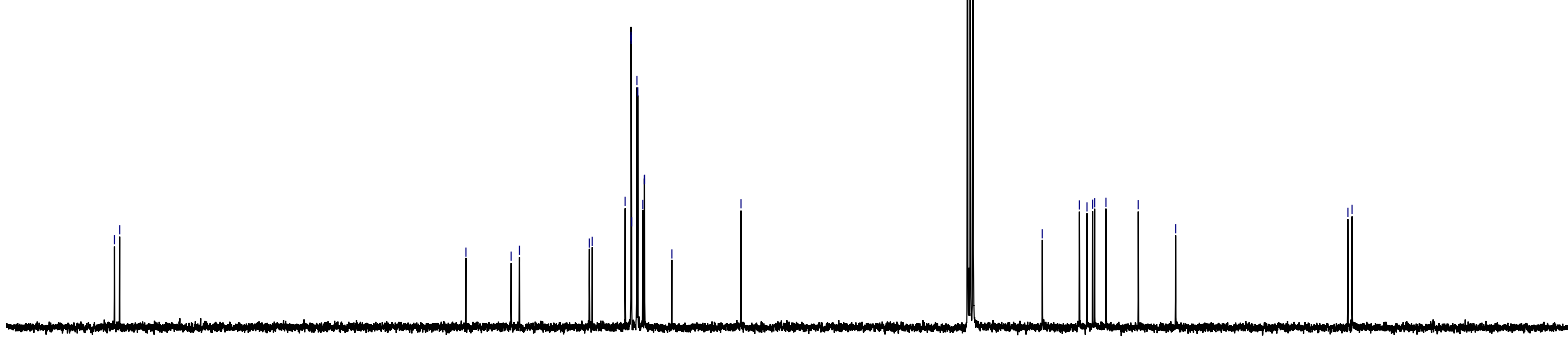

$\begin{array}{llllllllllll}220 & 210 & 200 & 190 & 180 & 170 & 160 & 150 & 140 & 130 & 120 & \begin{array}{c}110 \\ \mathrm{f} 1(\mathrm{ppm})\end{array}\end{array}$ 


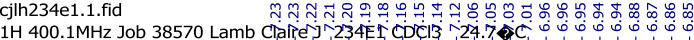
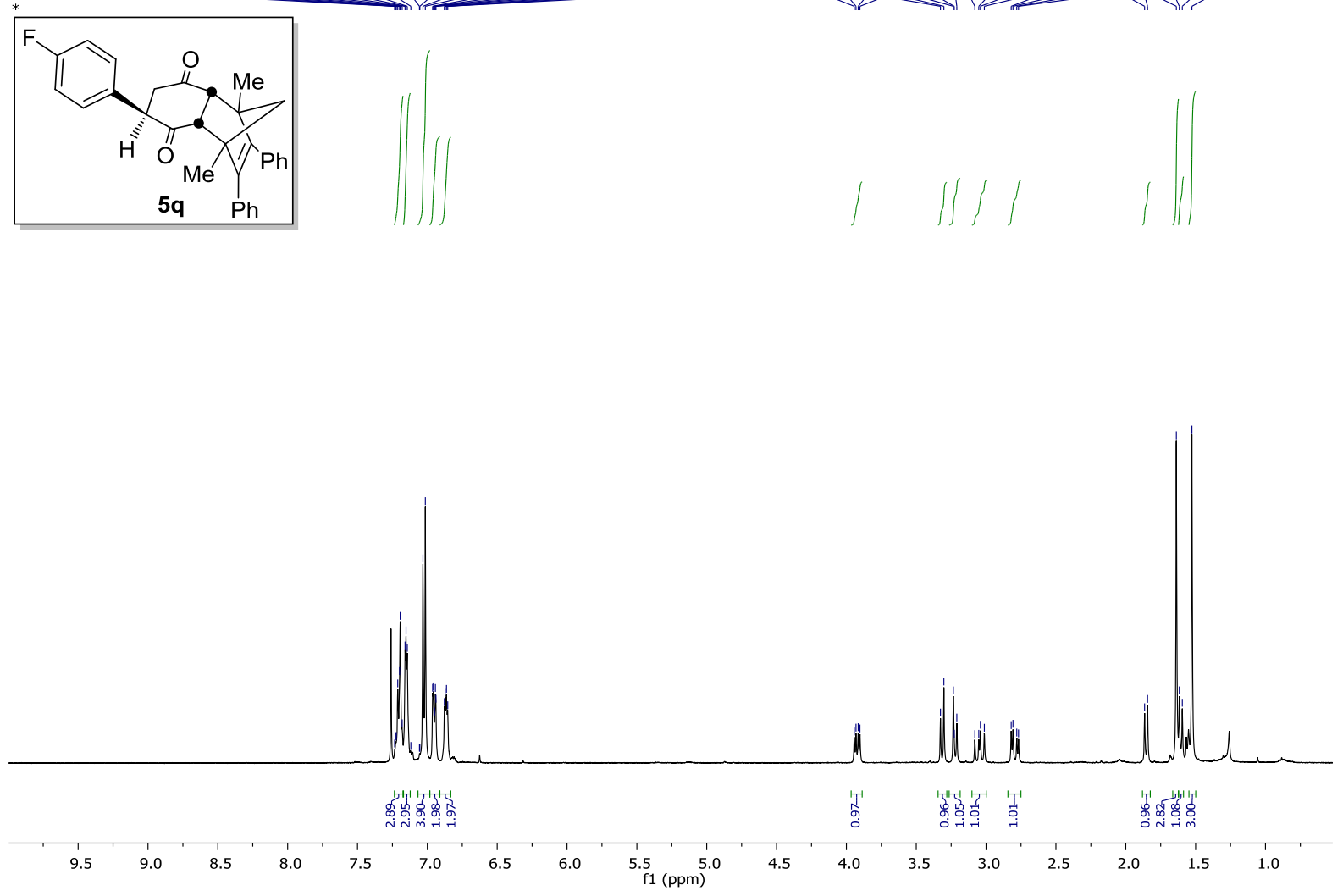

cjlf234b1b.1.fid

19F 376.5MHz Job 35253 Lamb Claire J 234B1B CDCl3 $25.0 \gg$ Chours $1 \mathrm{~min}$

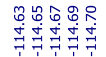

(I)

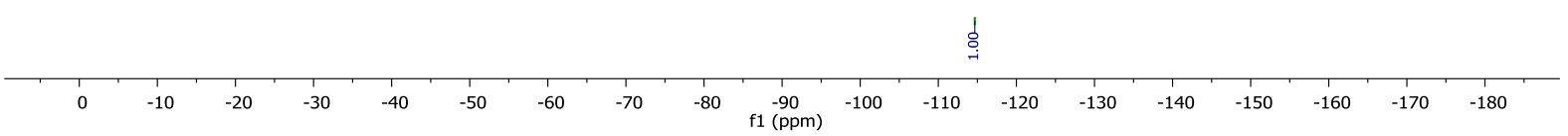




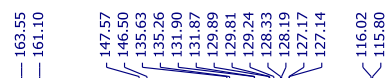

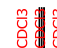

Oct26-2018.1.fid

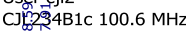

กับ

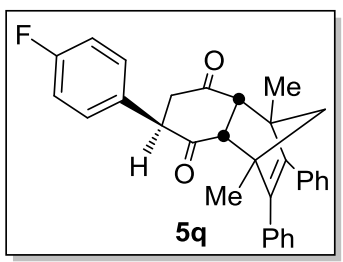

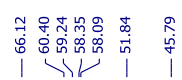

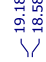

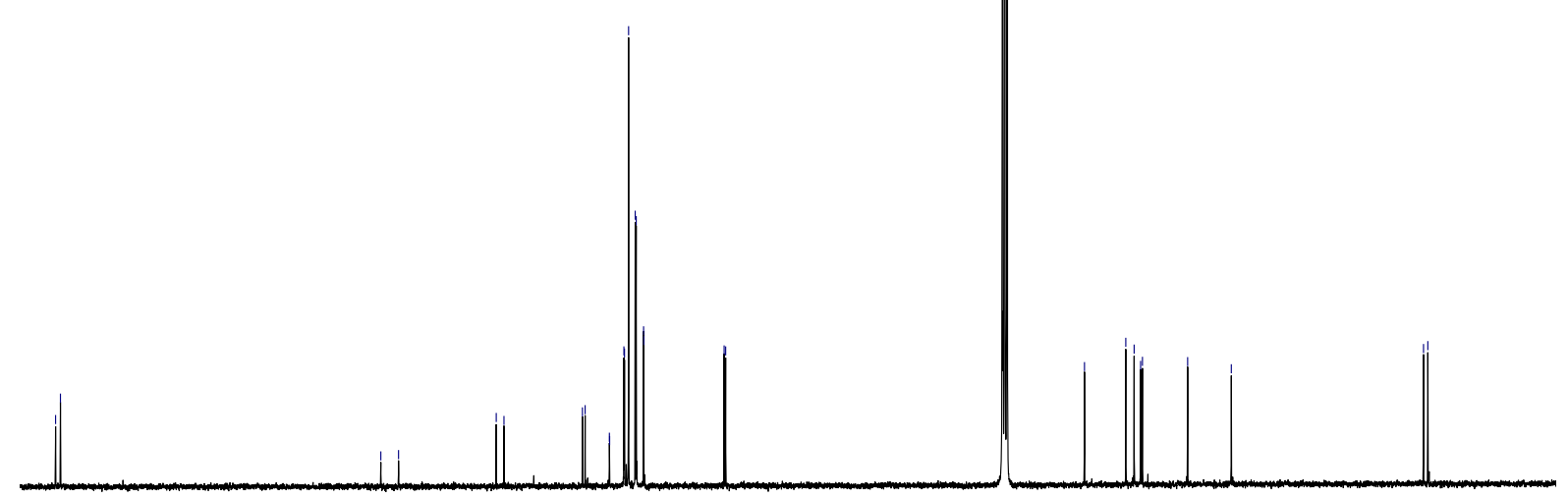

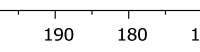

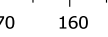

140

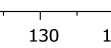

${ }_{\mathrm{f} 1(\mathrm{ppm})}^{100}$ 


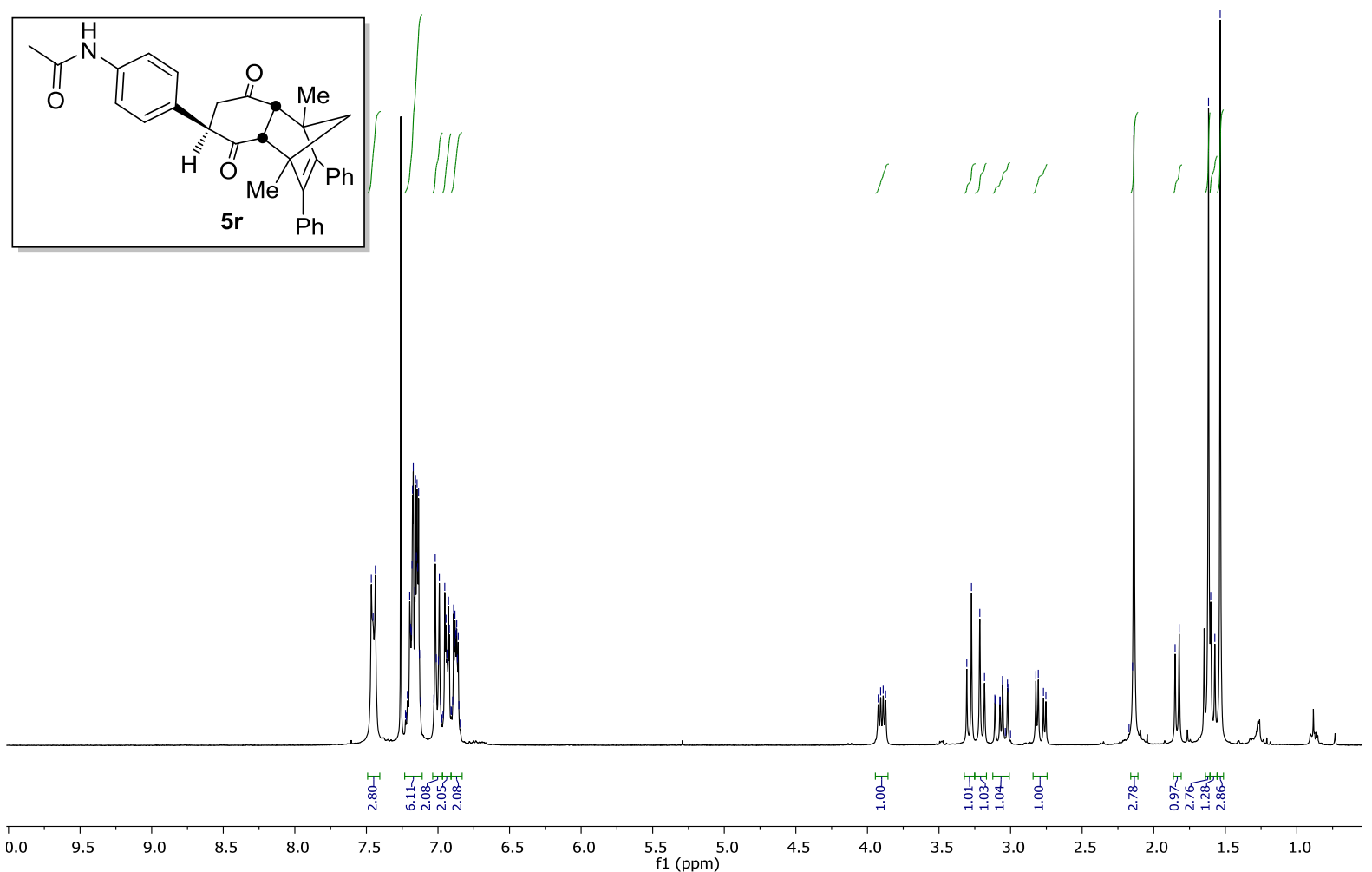

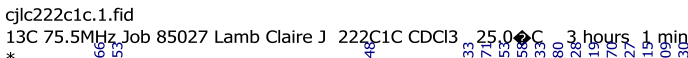

$\underbrace{\text { M. }}$
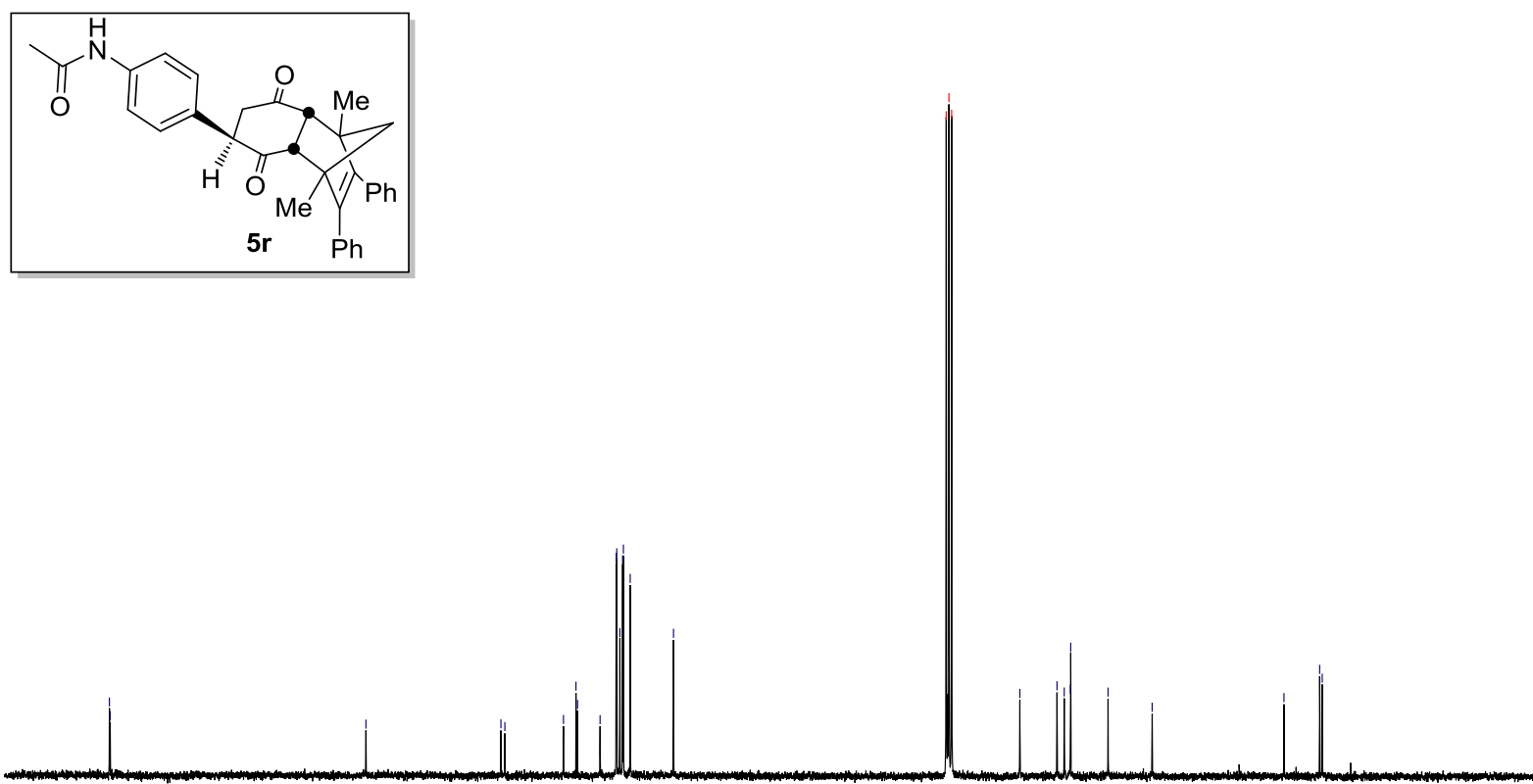

$\begin{array}{lllllllllllll}220 & 210 & 200 & 190 & 180 & 170 & 160 & 150 & 140 & 130 & 120 & \begin{array}{c}110 \\ \mathrm{f} 1(\mathrm{ppm})\end{array}\end{array}$ 


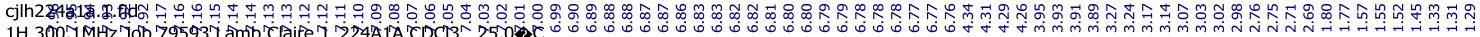

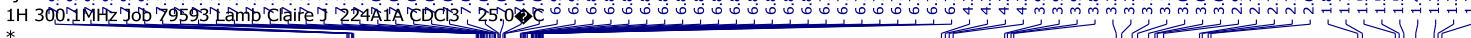
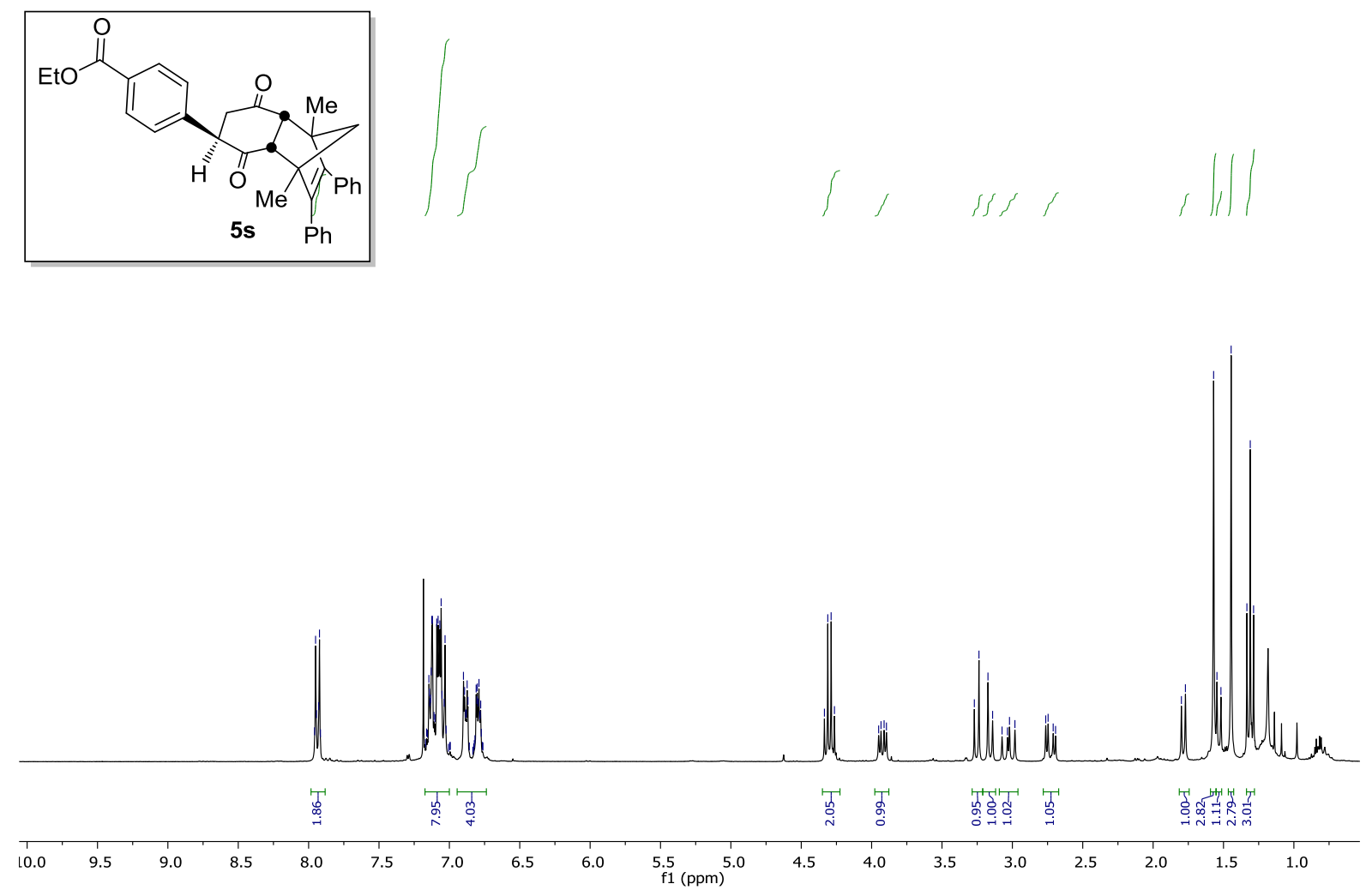

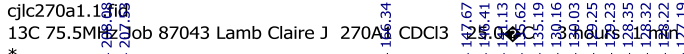

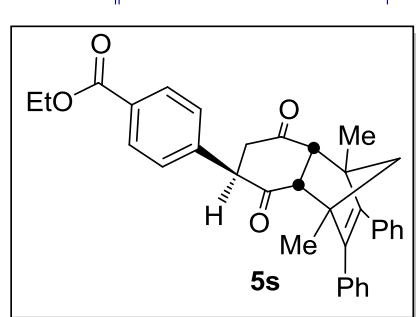

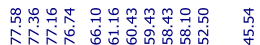

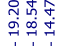

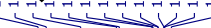

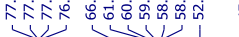

V

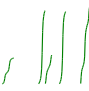




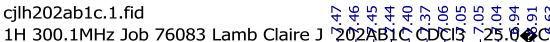

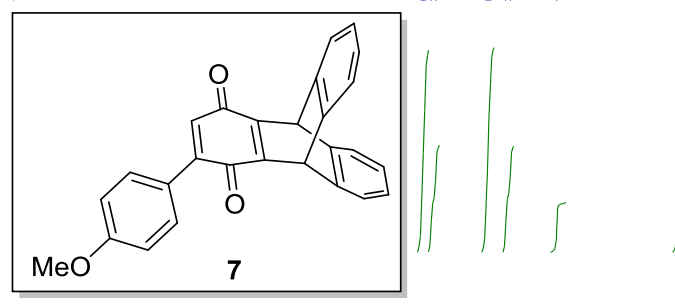

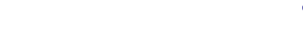

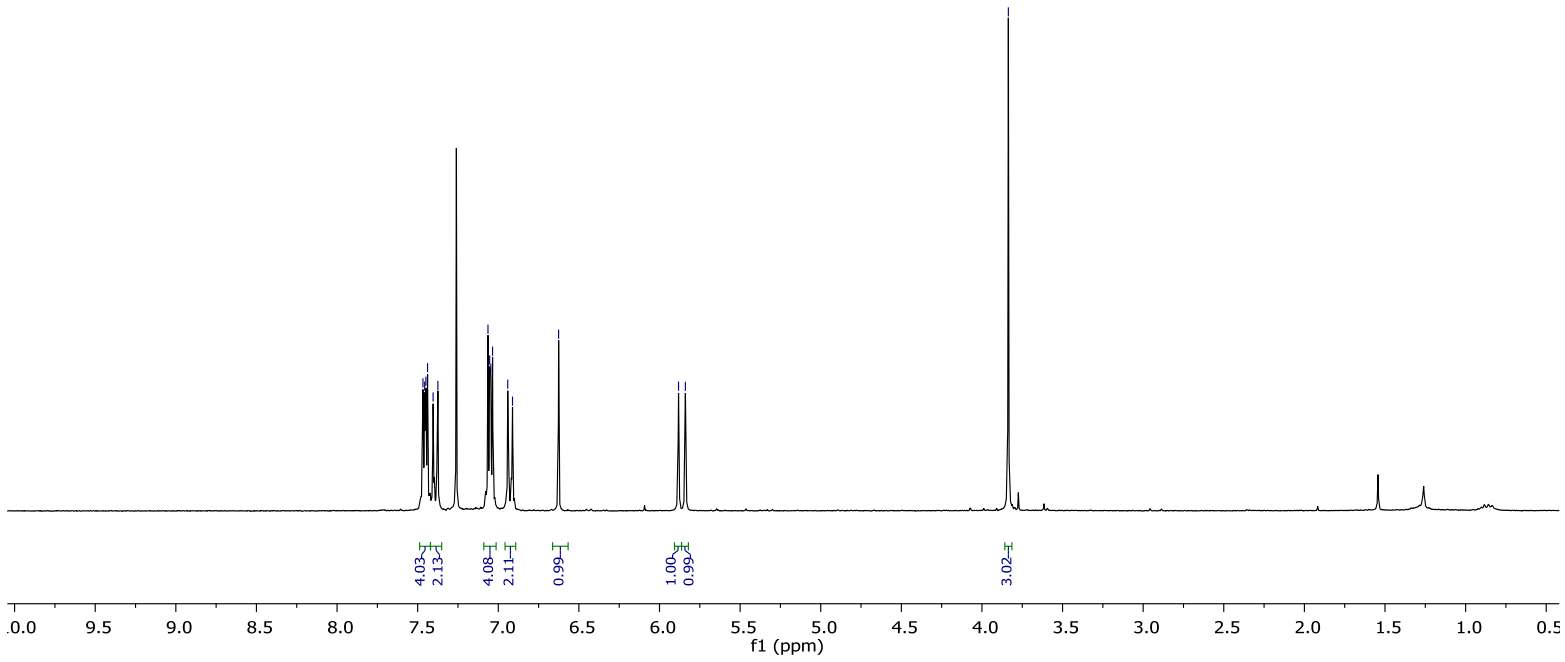

cjlc202ab1c.1.fid

13C 100.6MHz Job 32632 Lamb Claire J 202AB1C CDCl3 $25.42 \mathrm{C}$. 14 hours 20 min

$\checkmark$

|

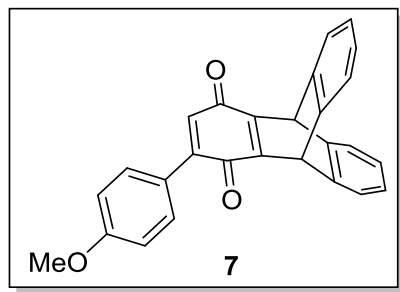

|

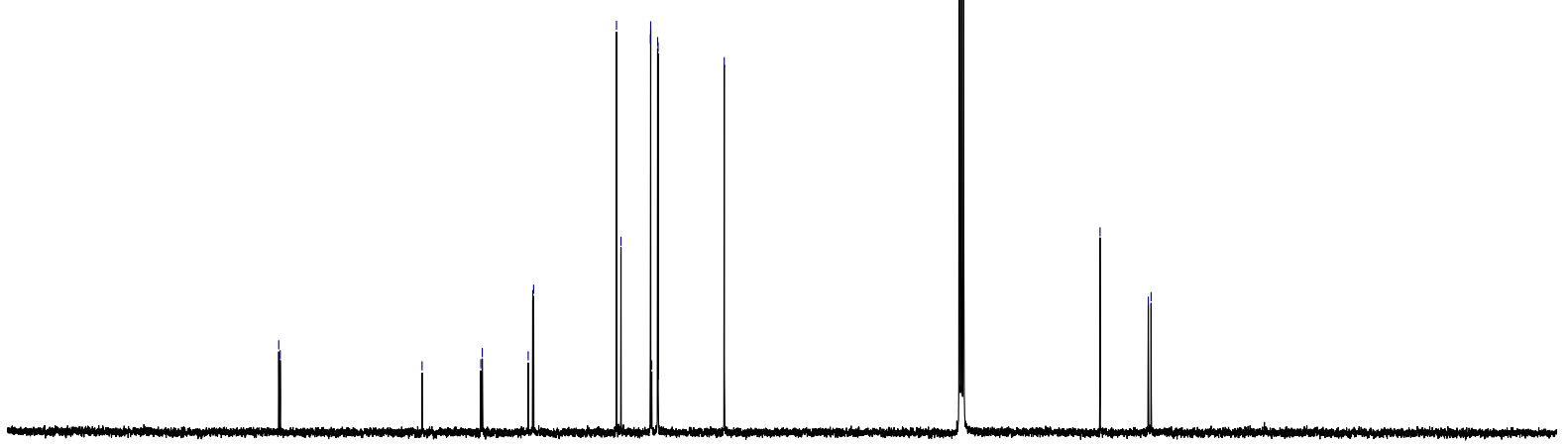

$\begin{array}{lllllllllllll}220 & 210 & 200 & 190 & 180 & 170 & 160 & 150 & 140 & 130 & 120 & 110 & 100 \\ f 1(\mathrm{ppm})\end{array}$ 


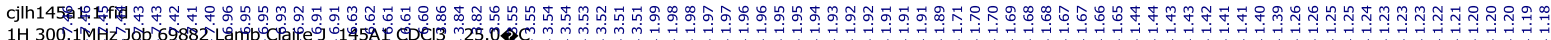
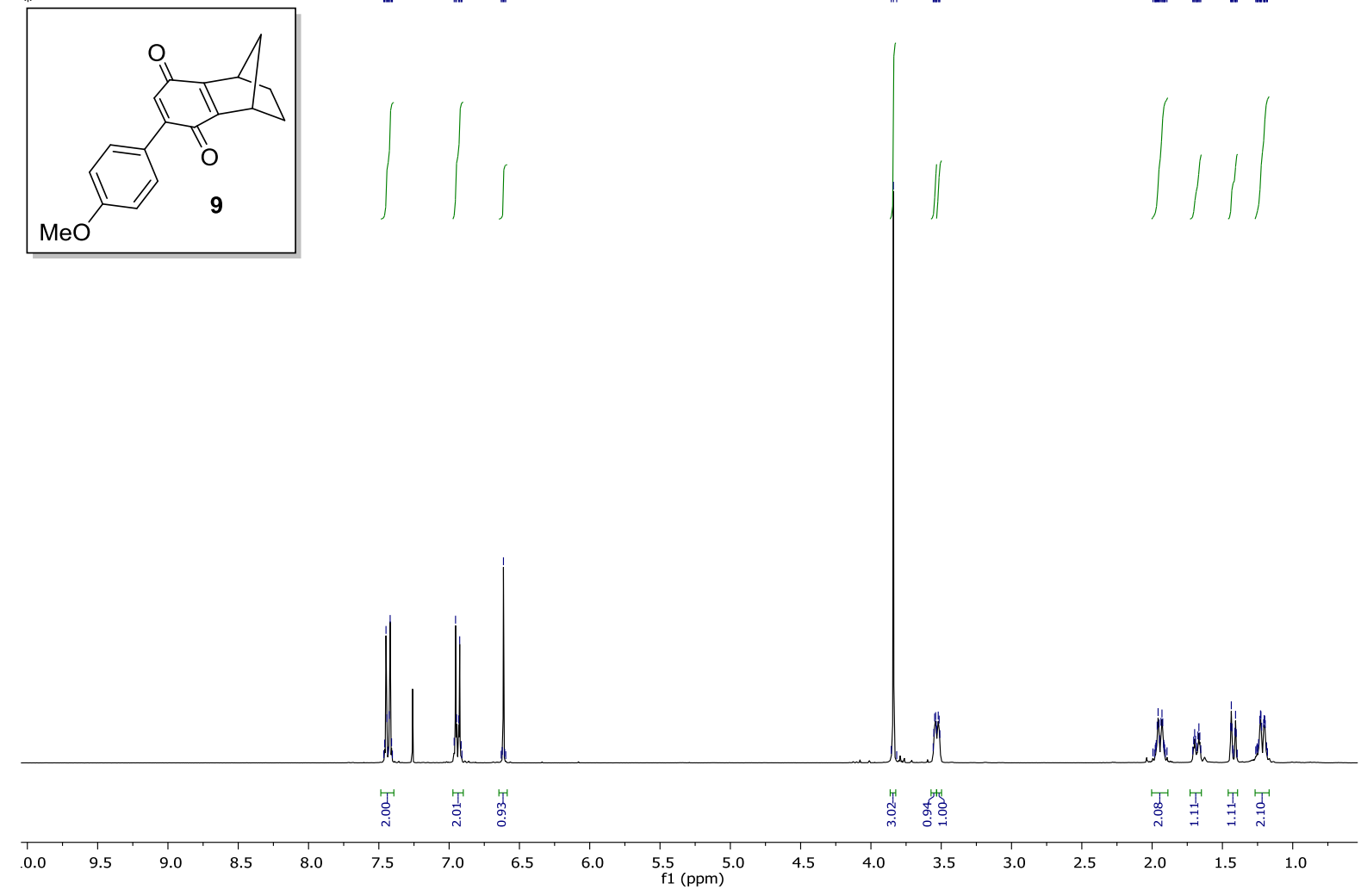

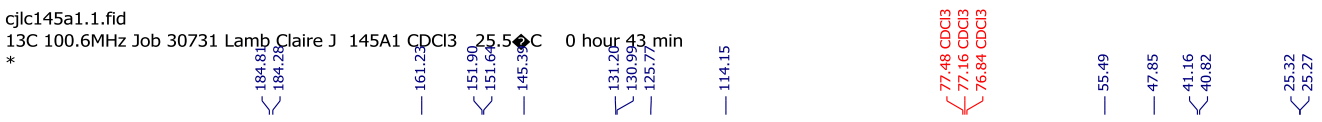
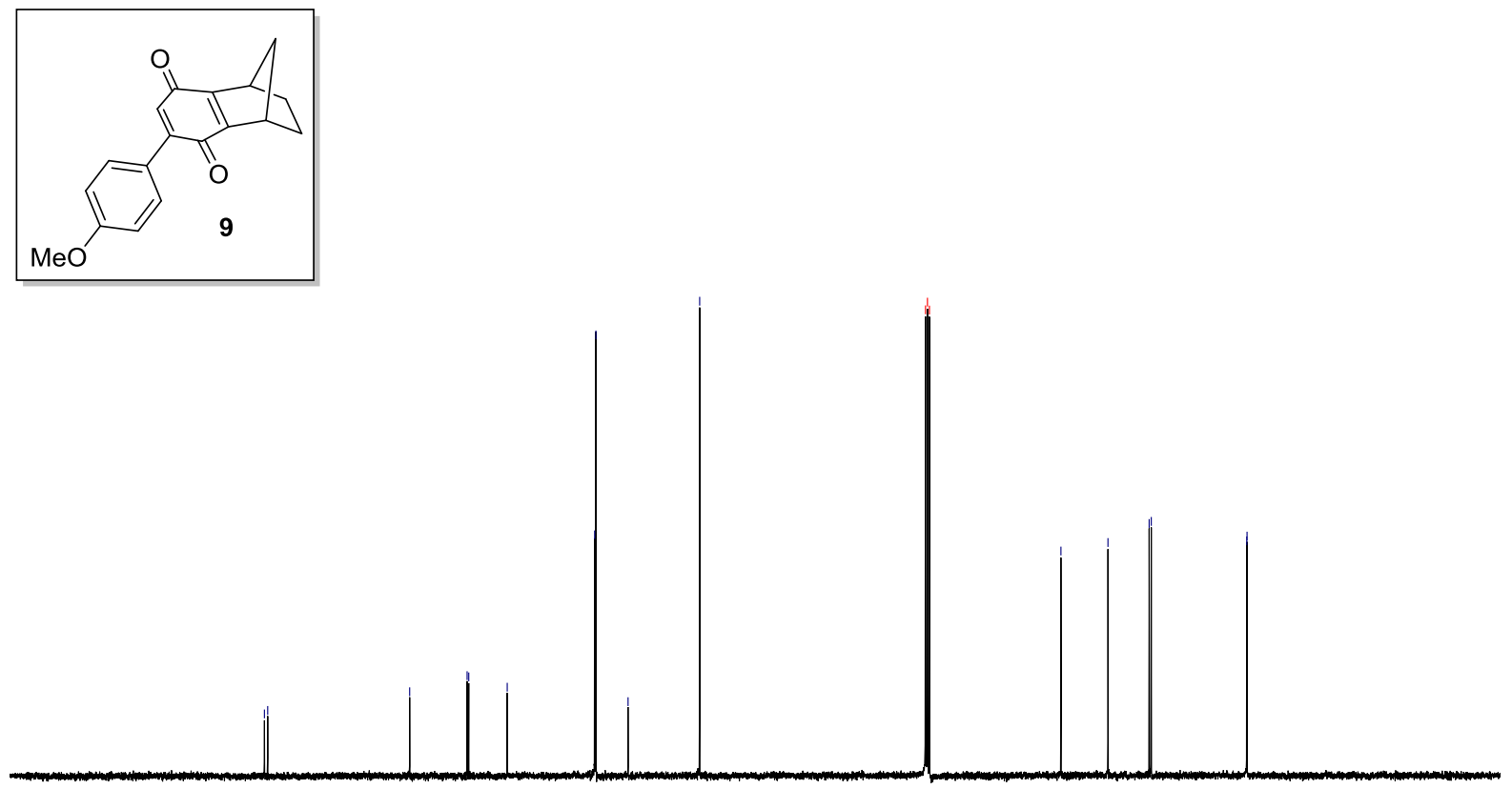

$\begin{array}{llllllllllllllllllllllllllllllllll}220 & 210 & 200 & 190 & 180 & 170 & 160 & 150 & 140 & 130 & 120 & 110 & 100 & 90 & 80 & 70 & 60 & 50 & 40 & 30 & 20 & 10 & 0 & -10\end{array}$ 